\title{
2-(Substituted amino)-8-azachromones from 4,6-Diaryl-2-pyridones: a Synthetic Strategy toward Compounds of Broad Structural Diversity
}

Steve Saulnier, Rayane Ghoteimi, Christophe Mathé, Suzanne Peyrottes and Jean-Pierre Uttaro*

Nucleosides \& Phosphorylated Effectors Team, Institut des Biomolécules Max Mousseron, UMR 5247 CNRS, Université de Montpellier, Campus Triolet, cc1705, Place Eugène Bataillon, 34095 Montpellier, France.

*Corresponding author,email: jean-pierre.uttaro@umontpellier.fr

\section{Table of Contents}

Copies of NMR spectra of 4-substituted chalcones

Copies of NMR spectra of compounds 1a-e-K

Copies of NMR spectra of compounds 2a-e

Copies of NMR spectra of compounds 3a-e

Copies of NMR spectra of compound $\mathbf{5 a}$

Copies of NMR spectra of compounds 4a-e

Copies of NMR spectra of compounds 6aa-am

Copies of NMR spectra of compounds 6ba-bc, 6ca-cc, 6da-db and 6ea-eb 


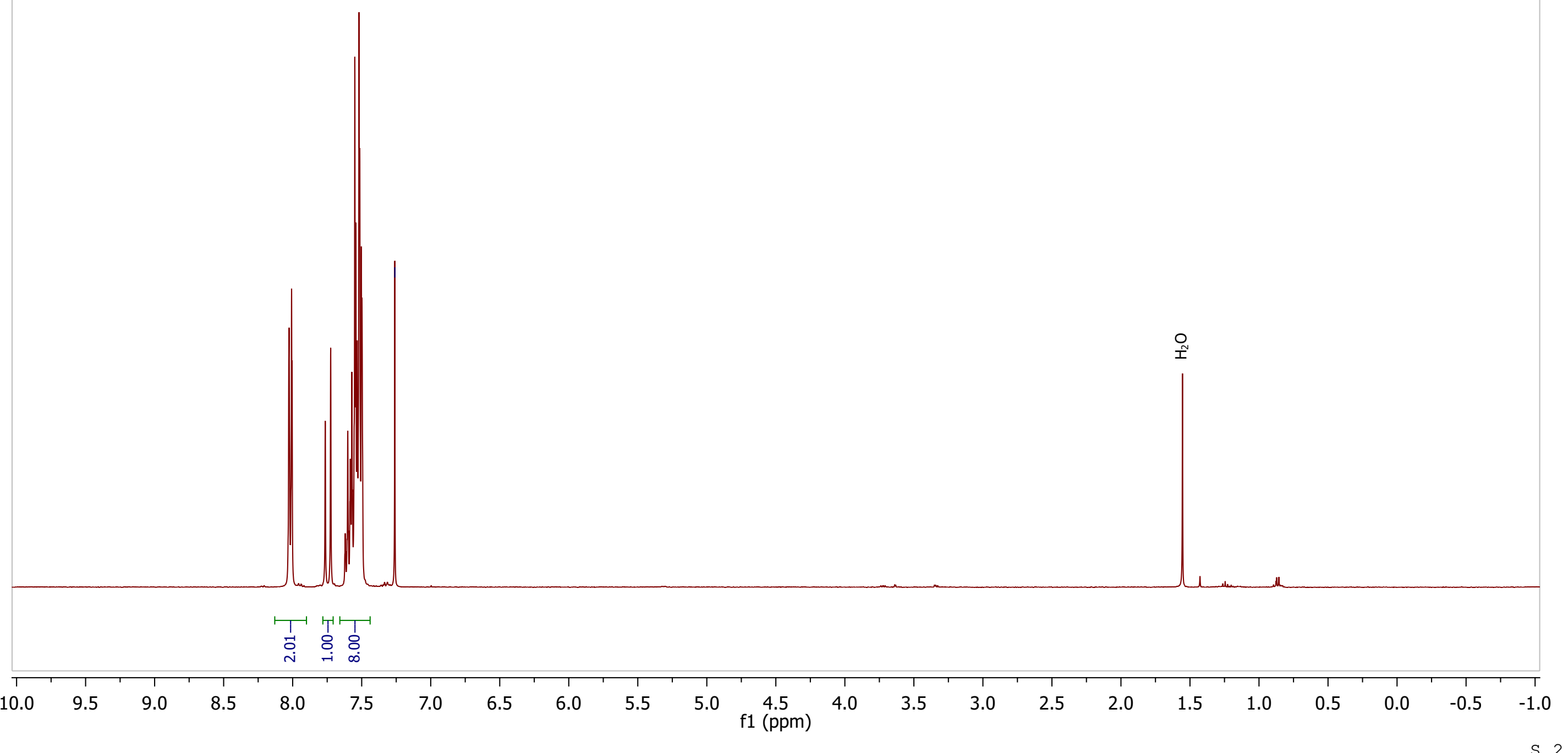



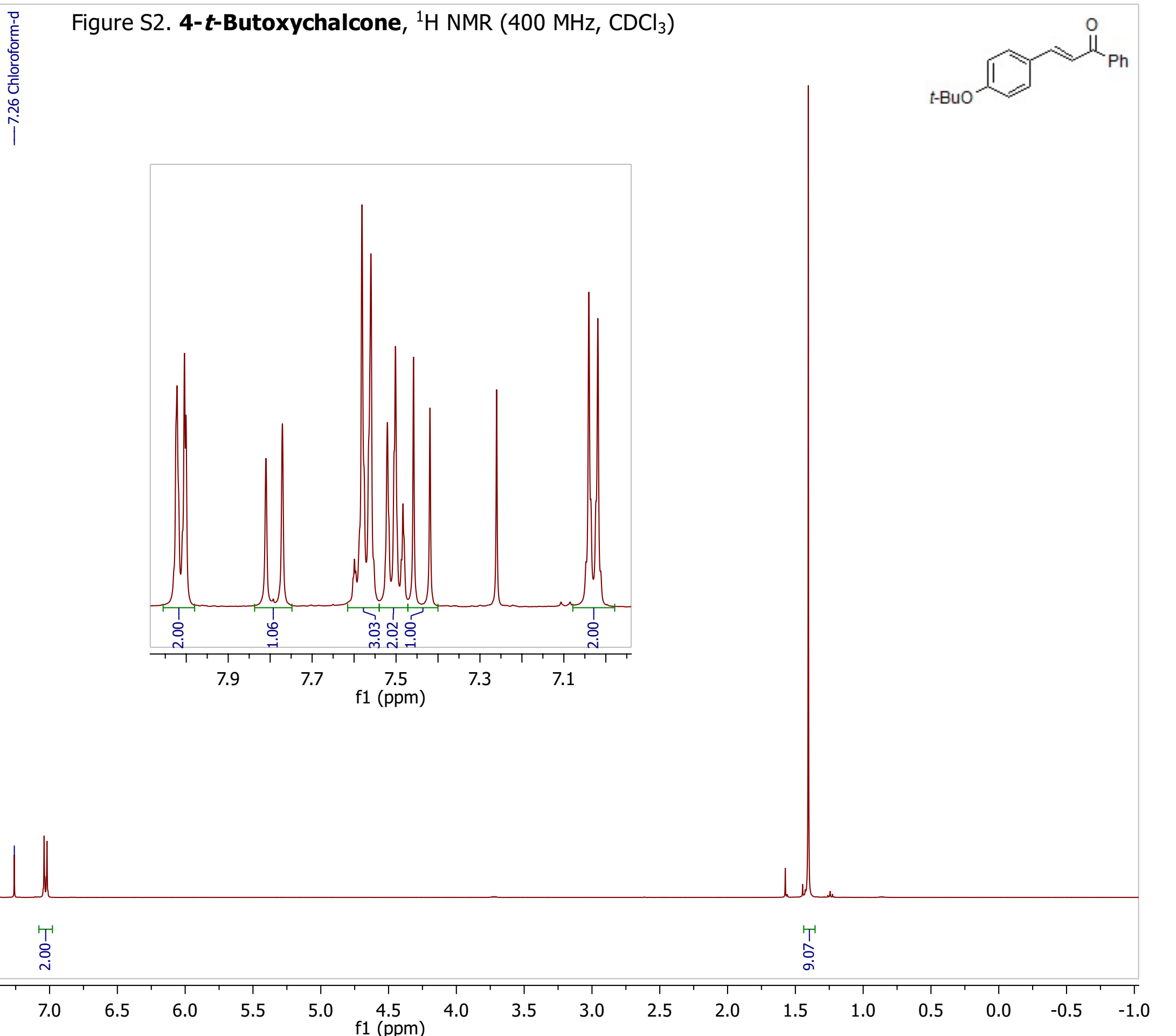
Figure S3. 4- $t$-Butoxychalcone, ${ }^{13} \mathrm{C}\left\{{ }^{1} \mathrm{H}\right\}$ NMR $\left(101 \mathrm{MHz}, \mathrm{CDCl}_{3}\right)$

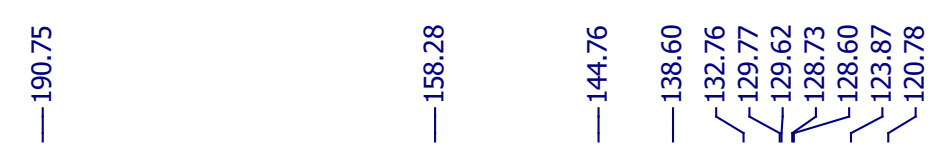

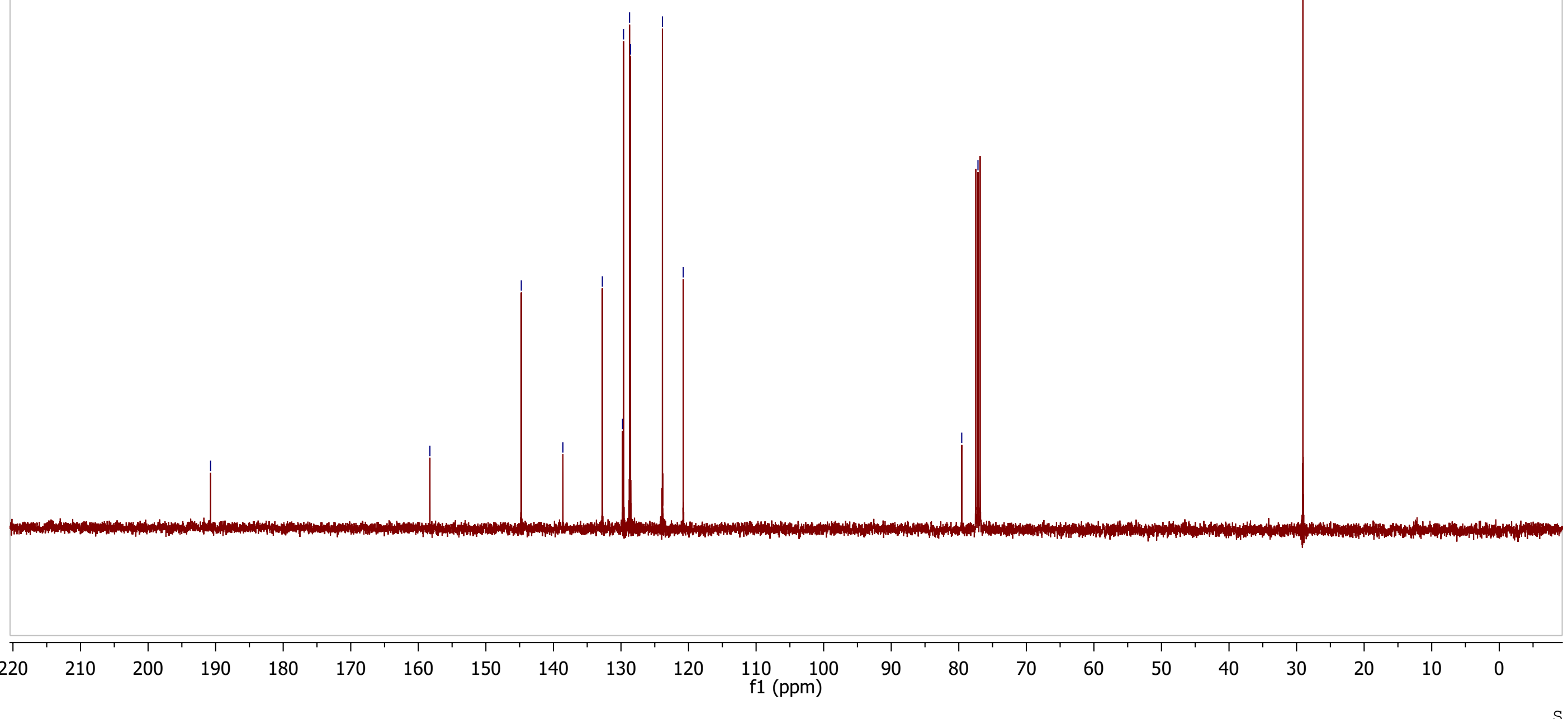



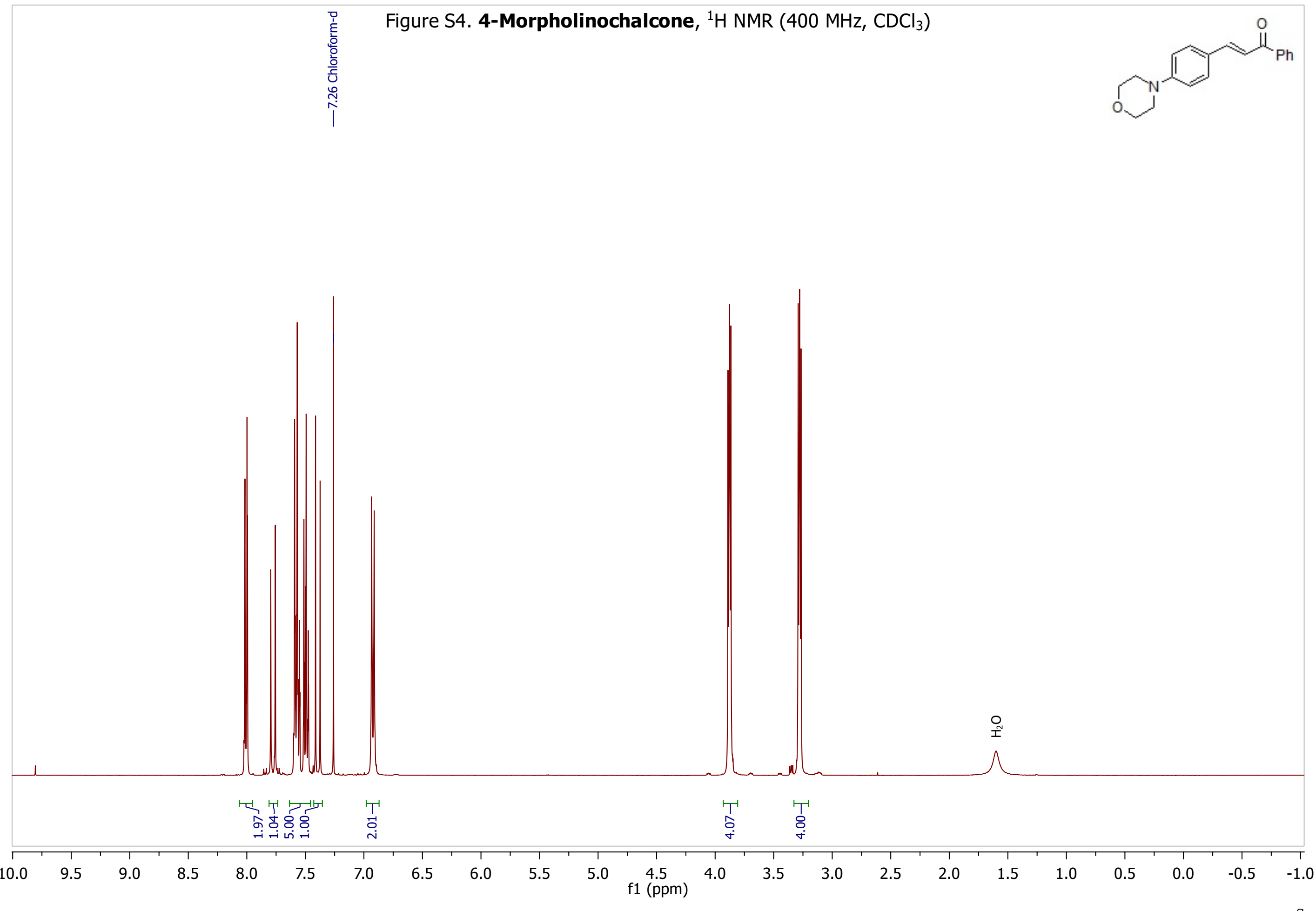
Figure S5. 4-Morpholinochalcone, ${ }^{13} \mathrm{C}\left\{{ }^{1} \mathrm{H}\right\} \mathrm{NMR}\left(101 \mathrm{MHz}, \mathrm{CDCl}_{3}\right)$
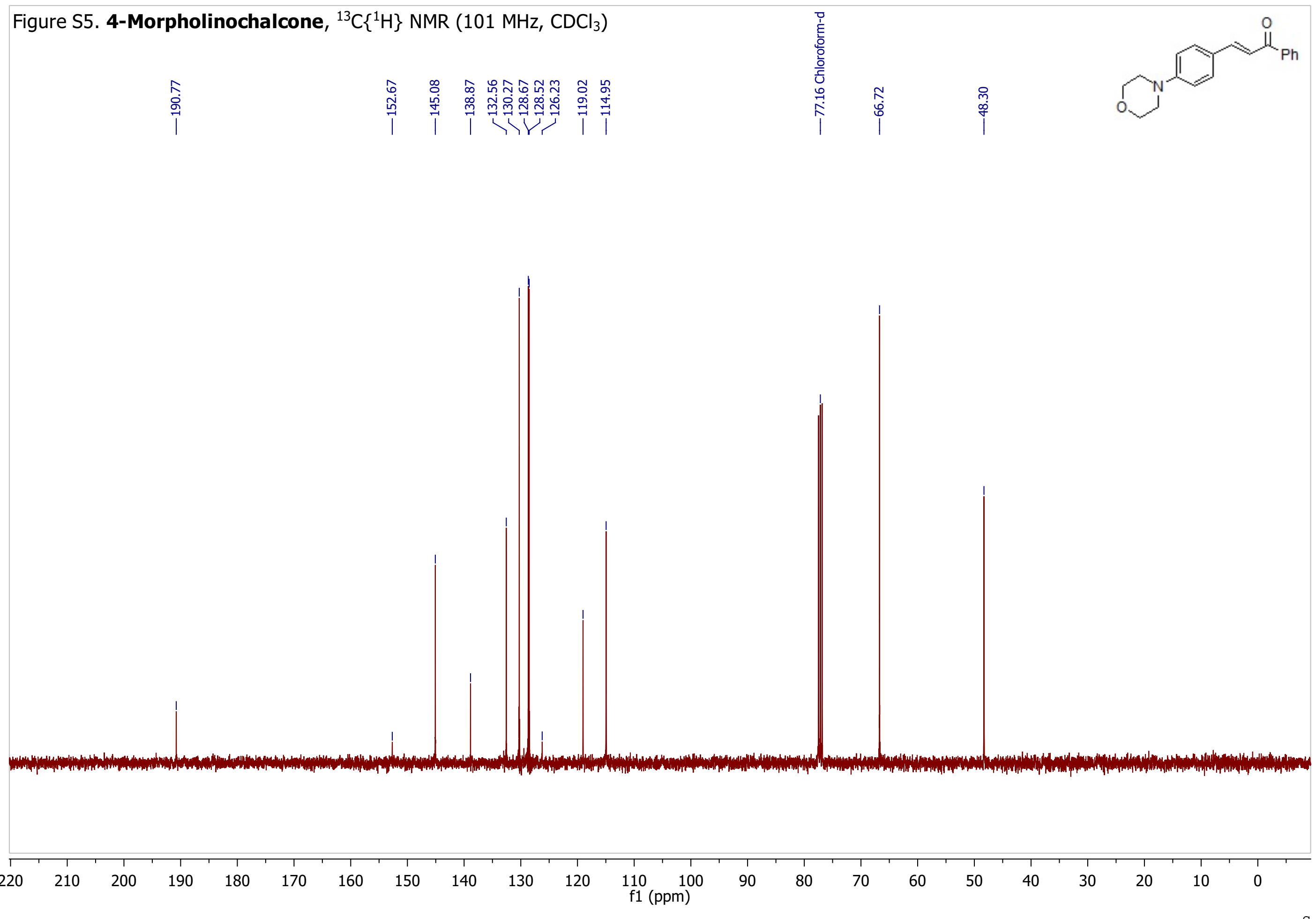


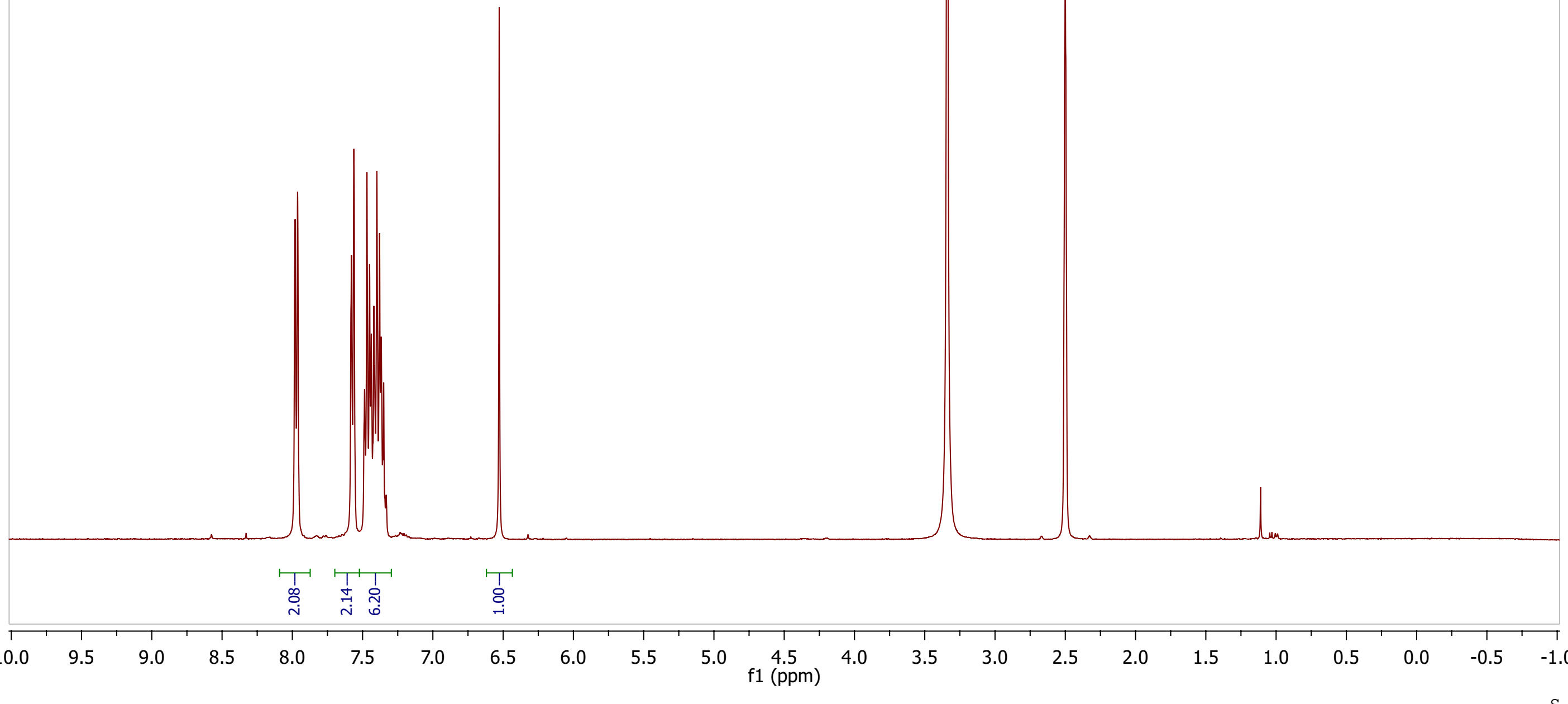


Figure S7. Compound $\mathbf{1 a - K},{ }^{13} \mathrm{C}\left\{{ }^{1} \mathrm{H}\right\}$ NMR (101 MHz, DMSO- $\mathrm{d}_{6}$ )

| l|l |
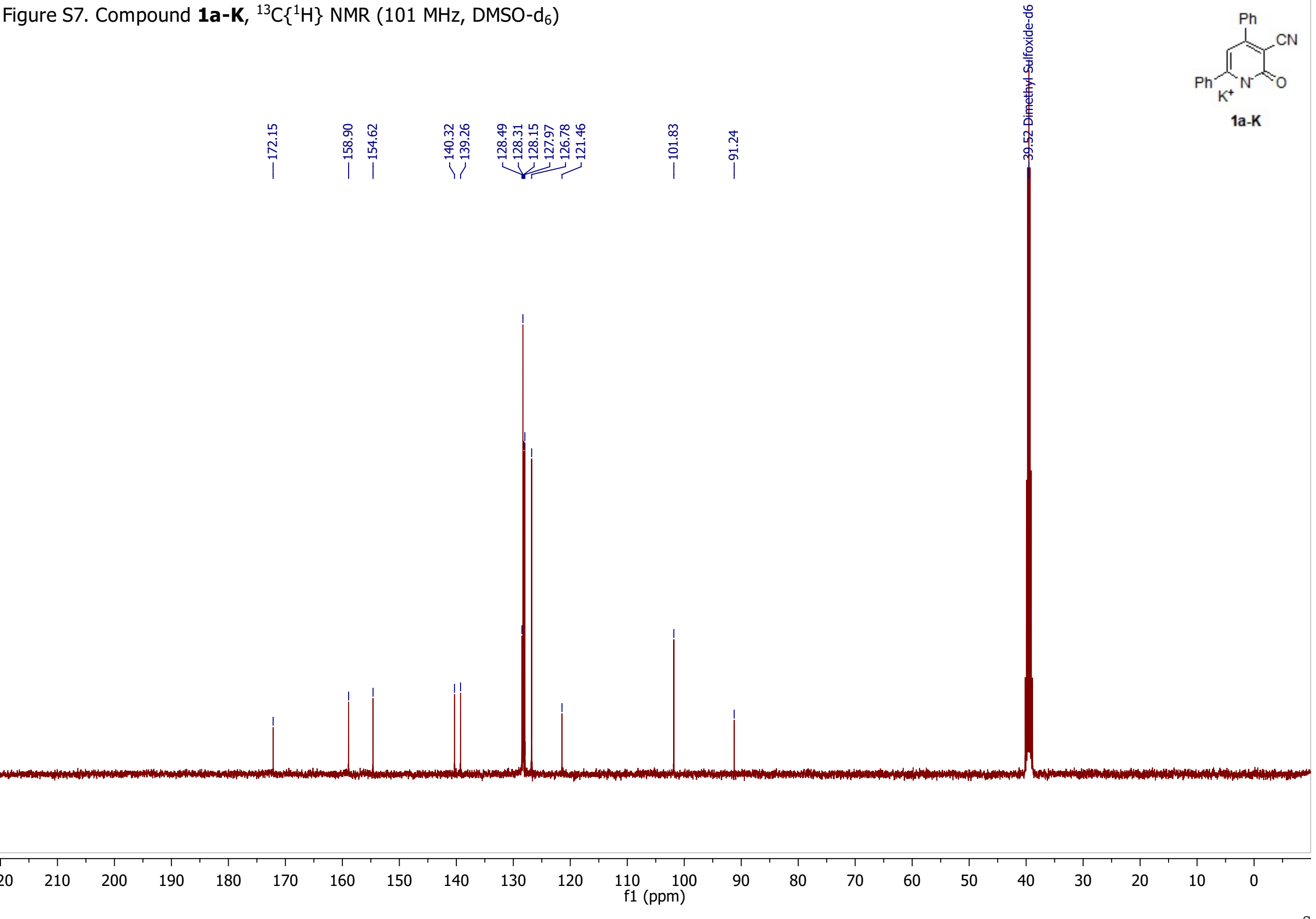
Figure S8. Compound 1b-K, ${ }^{1} \mathrm{H}$ NMR (400 MHz, DMSO-d 6 )
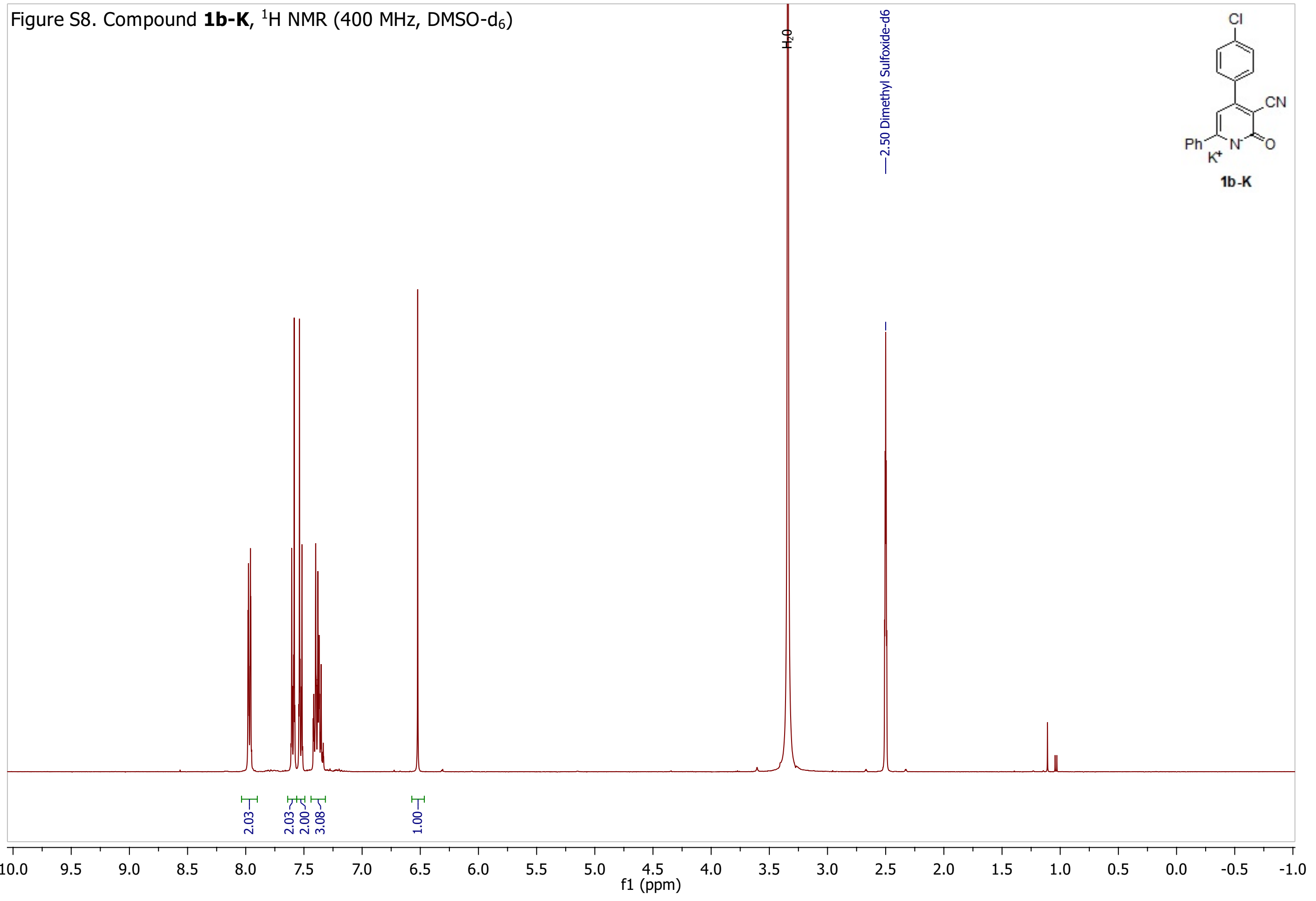
Figure S9. Compound 1b-K, ${ }^{13} \mathrm{C}\left\{{ }^{1} \mathrm{H}\right\}$ NMR (101 MHz, DMSO- $\mathrm{d}_{6}$ )

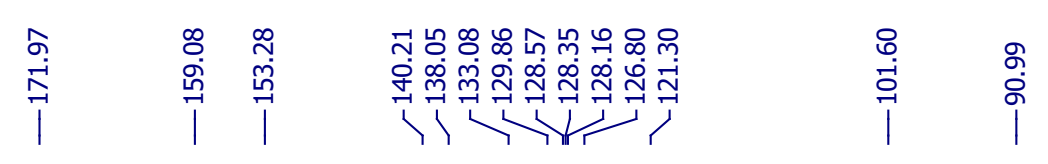
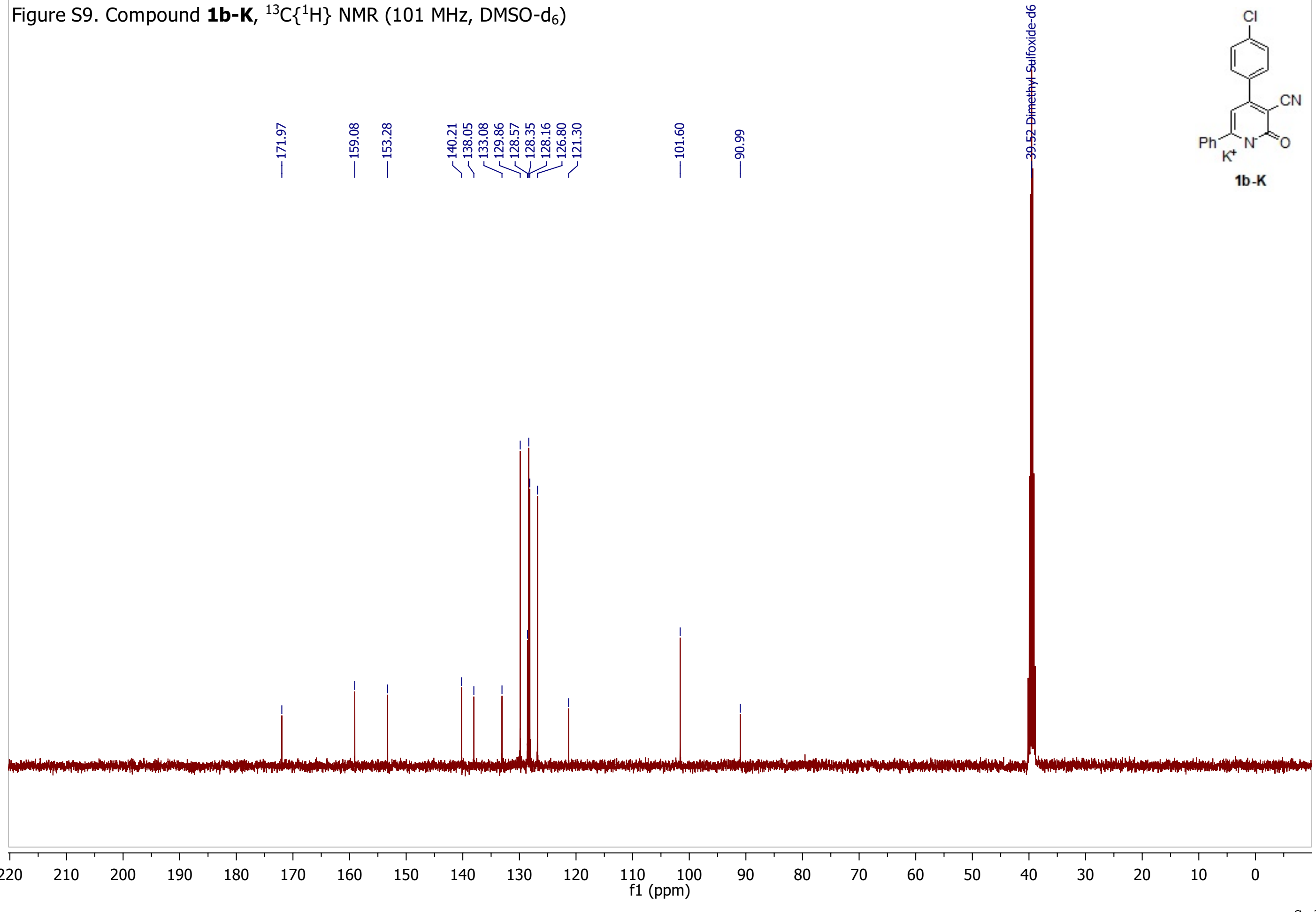

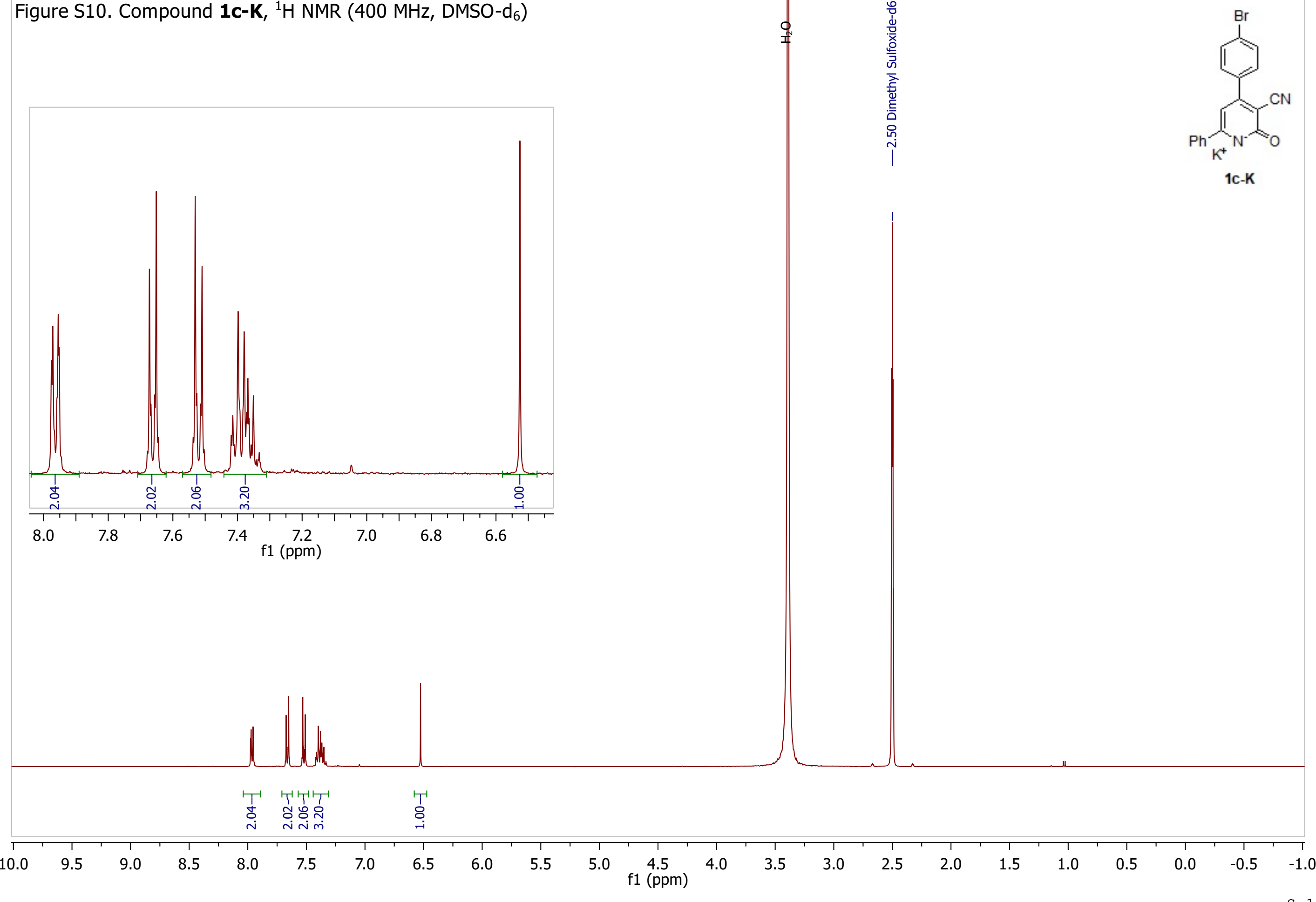


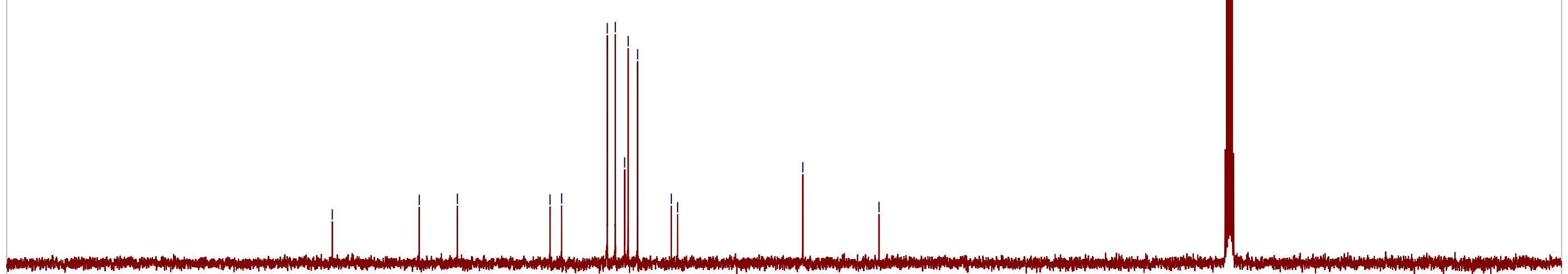

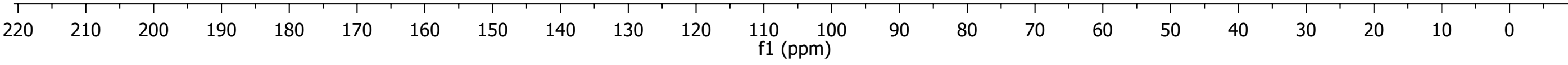


Figure S12. Compound 1d-K, ${ }^{1} \mathrm{H}$ NMR (400 MHz, DMSO-d 6 )
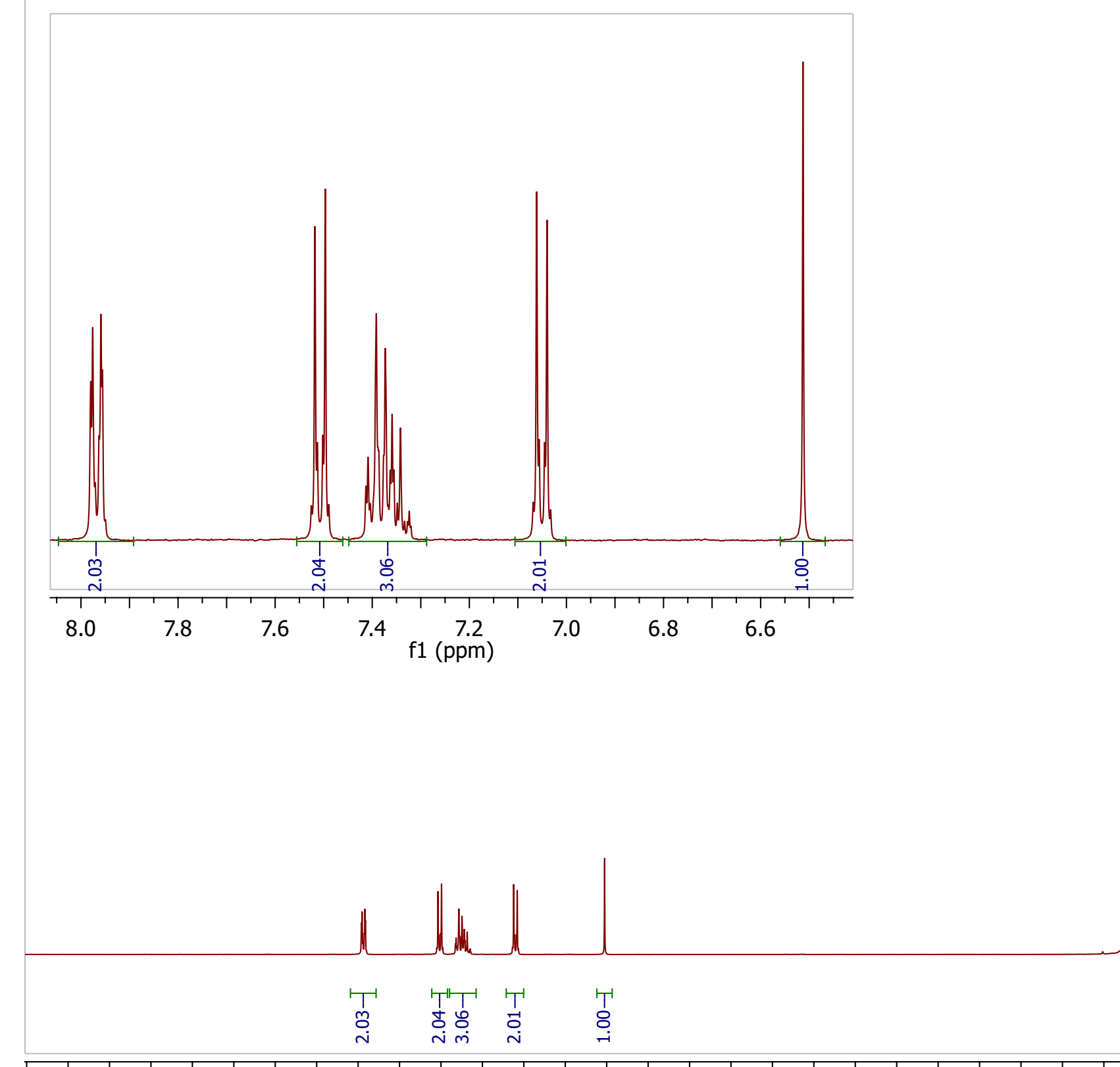

\begin{tabular}{|c|c|c|c|c|c|c|c|c|c|c|c|c|c|c|c|c|c|c|c|c|c|}
\hline & & & 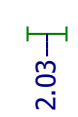 & 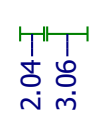 & $\begin{array}{l}\underset{T}{T} \\
\stackrel{\sim}{\sim}\end{array}$ & $\begin{array}{l}\text { T' } \\
\text { ه } \\
\end{array}$ & & & & & & & & & & 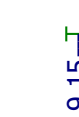 & & & & & \\
\hline 1 & 1 & 1 & 1 & 1 & 1 & 1 & 1 & 1 & 1 & 1 & 1 & 1 & 1 & 1 & 1 & 1 & 1 & 1 & 1 & 1 & \\
\hline 9.5 & 9.0 & 8.5 & 8.0 & 7.5 & 7.0 & 6.5 & 6.0 & 5.5 & 5.0 & $\begin{array}{c}4.5 \\
\text { f1 }(\mathrm{ppm})\end{array}$ & 4.0 & 3.5 & 3.0 & 2.5 & 2.0 & 1.5 & 1.0 & 0.5 & 0.0 & -0.5 & -1.0 \\
\hline
\end{tabular}


Figure S13. Compound 1d-K, ${ }^{13} \mathrm{C}\left\{{ }^{1} \mathrm{H}\right\}$ NMR (101 MHz, DMSO-d 6 )

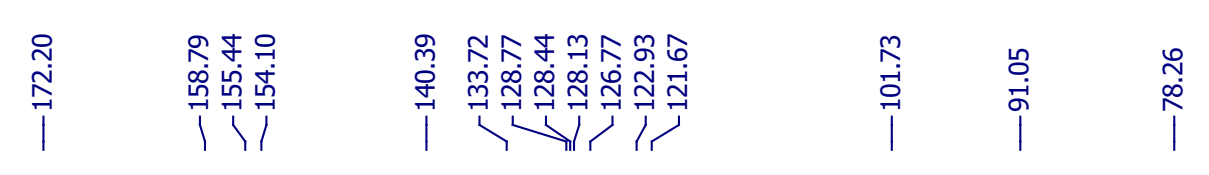
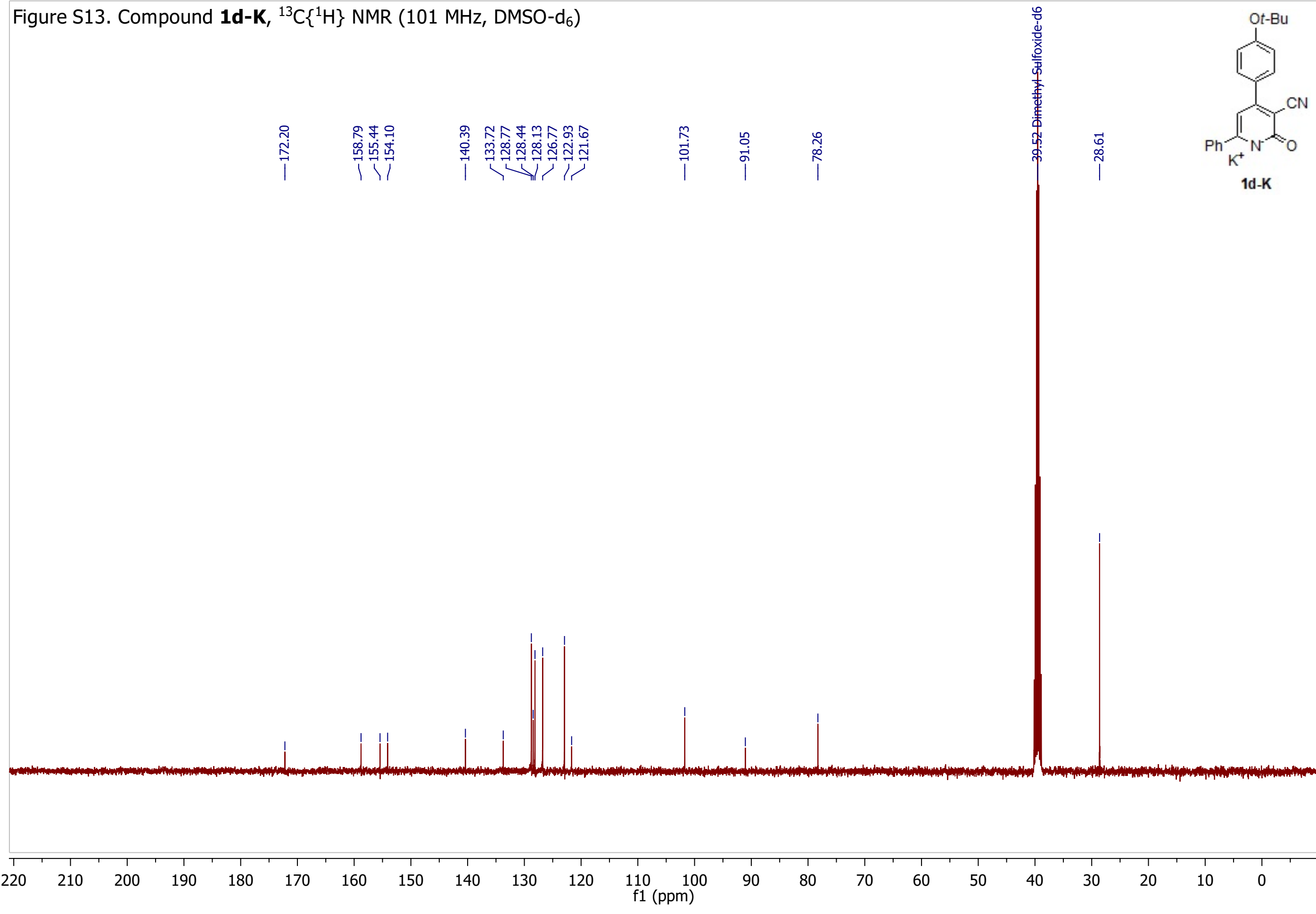

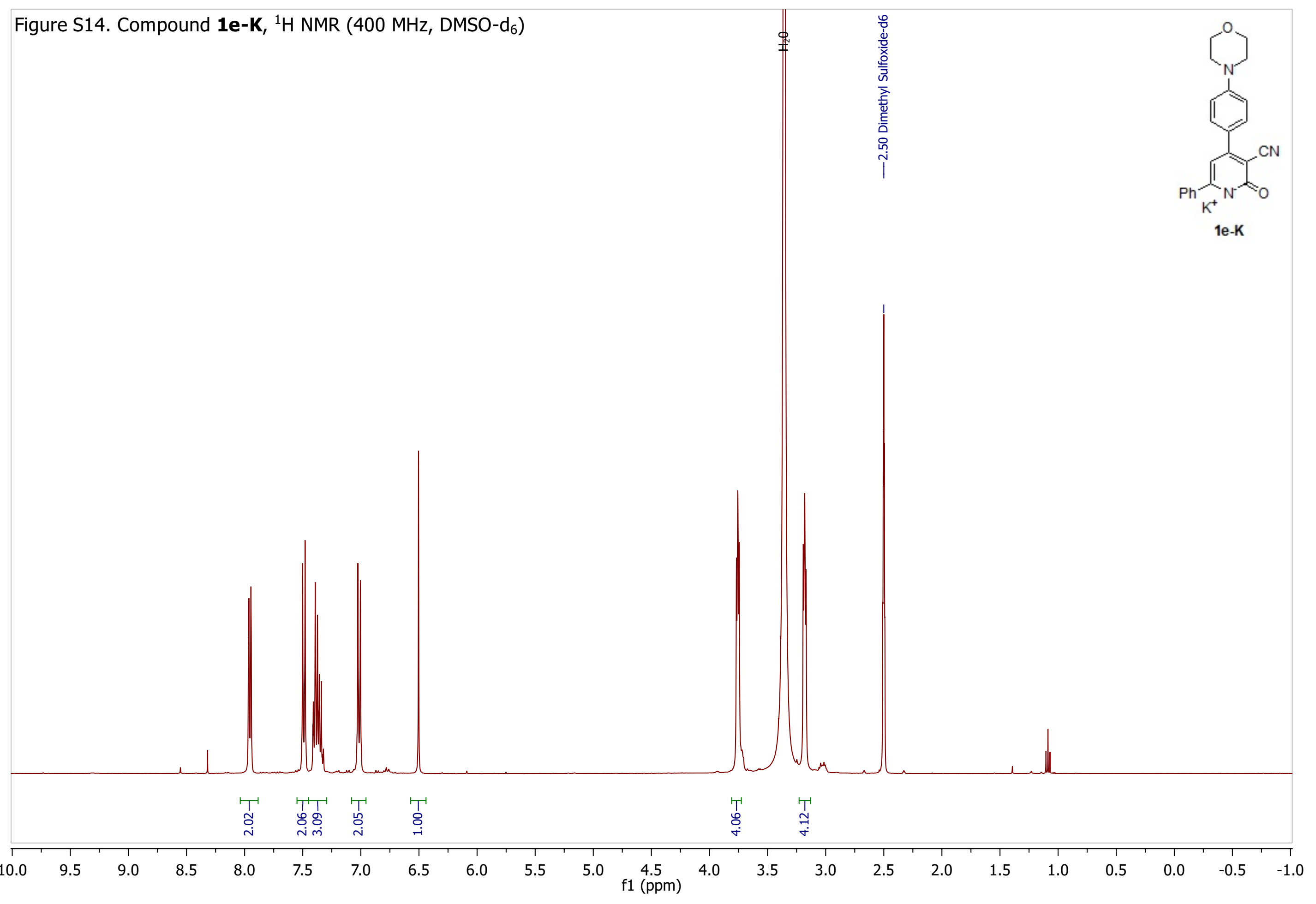
Figure S15. Compound $\mathbf{1 e - K},{ }^{13} \mathrm{C}\left\{{ }^{1} \mathrm{H}\right\}$ NMR (101 MHz, DMSO-d 6 )
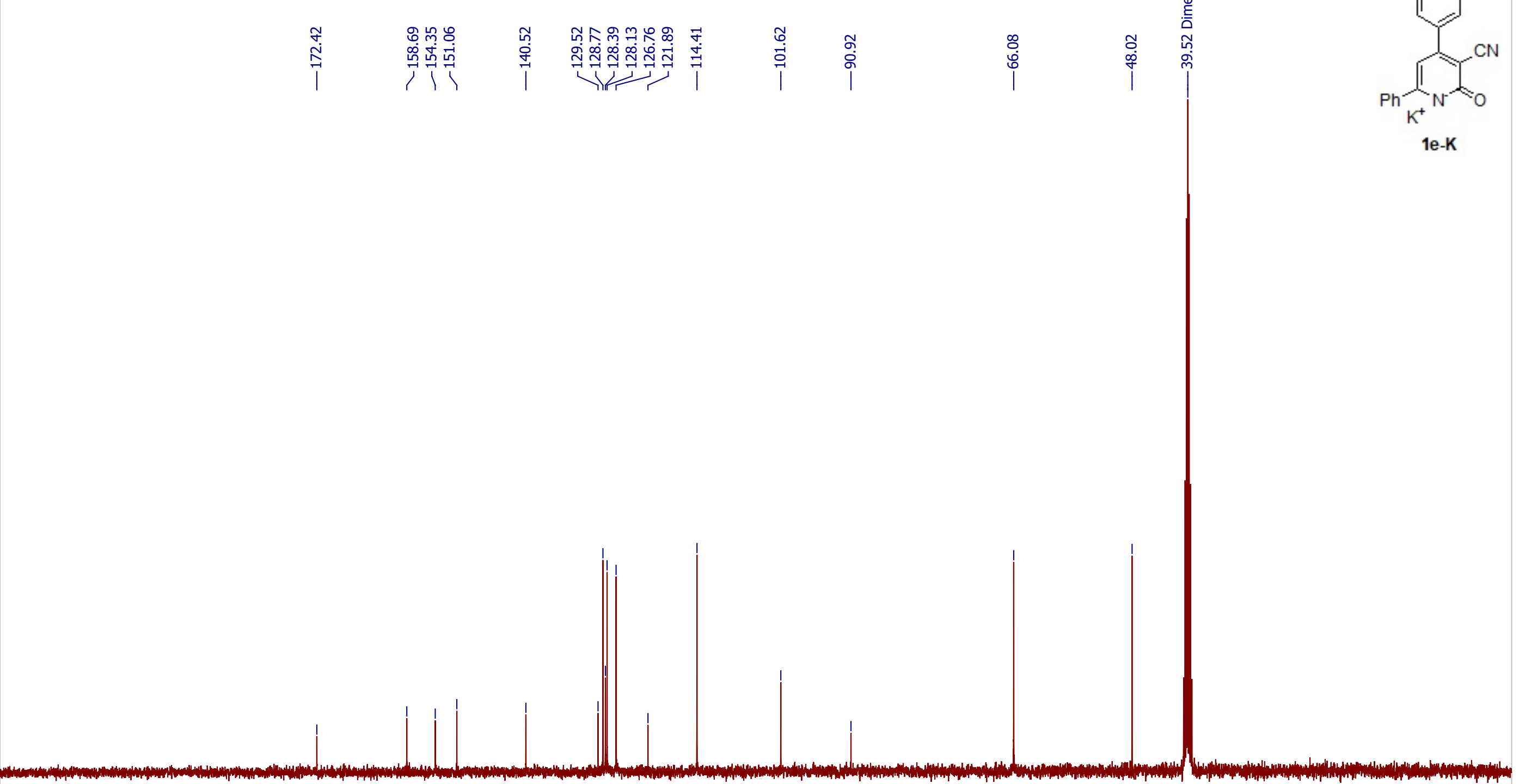

\begin{tabular}{|c|c|c|c|c|c|c|c|c|c|c|c|c|c|c|c|c|c|c|c|c|c|}
\hline$T$ & 1 & 1 & 1 & 1 & 1 & 1 & 1 & 1 & 1 & 1 & $1 \quad 1 \quad 1$ & 1 & 1 & 1 & 1 & 1 & 1 & 1 & $T$ & 1 & $T$ \\
\hline 220 & 210 & 200 & 190 & 180 & 170 & 160 & 150 & 140 & 130 & 120 & $110 \quad 100$ & 90 & 80 & 70 & 60 & 50 & 40 & 30 & 20 & 10 & 0 \\
\hline
\end{tabular}


Figure S16. Compound 2a, ${ }^{1} \mathrm{H}$ NMR (400 MHz, $\left.\mathrm{CDCl}_{3}\right)$

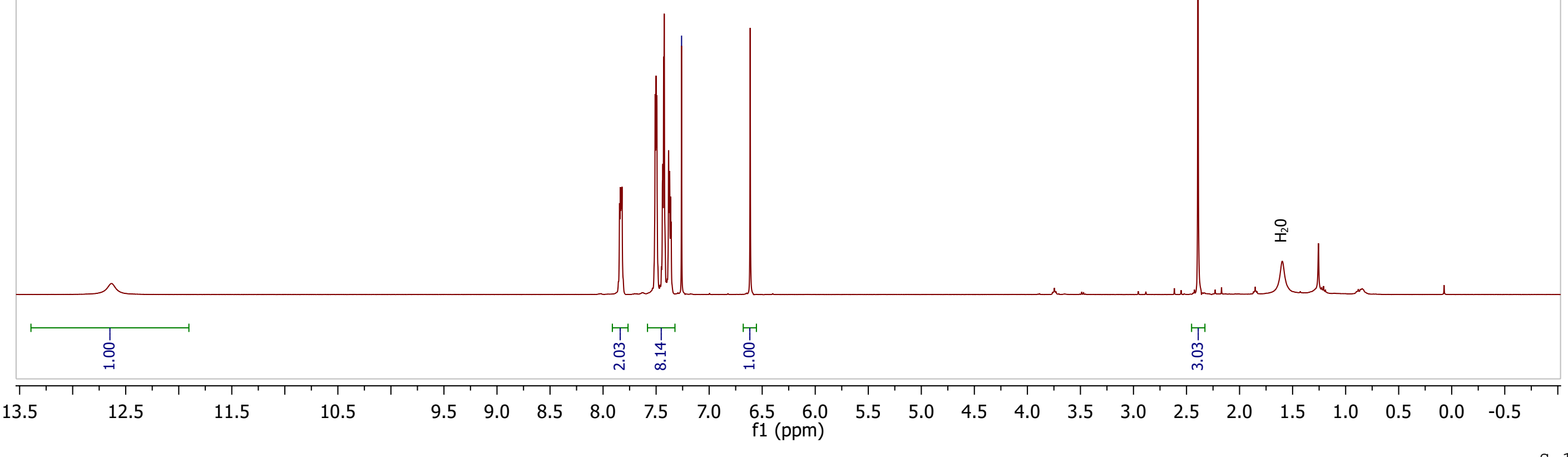


Figure S17. Compound $\mathbf{2 b},{ }^{1} \mathrm{H}$ NMR $\left(500 \mathrm{MHz} \mathrm{CDCl}_{3}\right)$
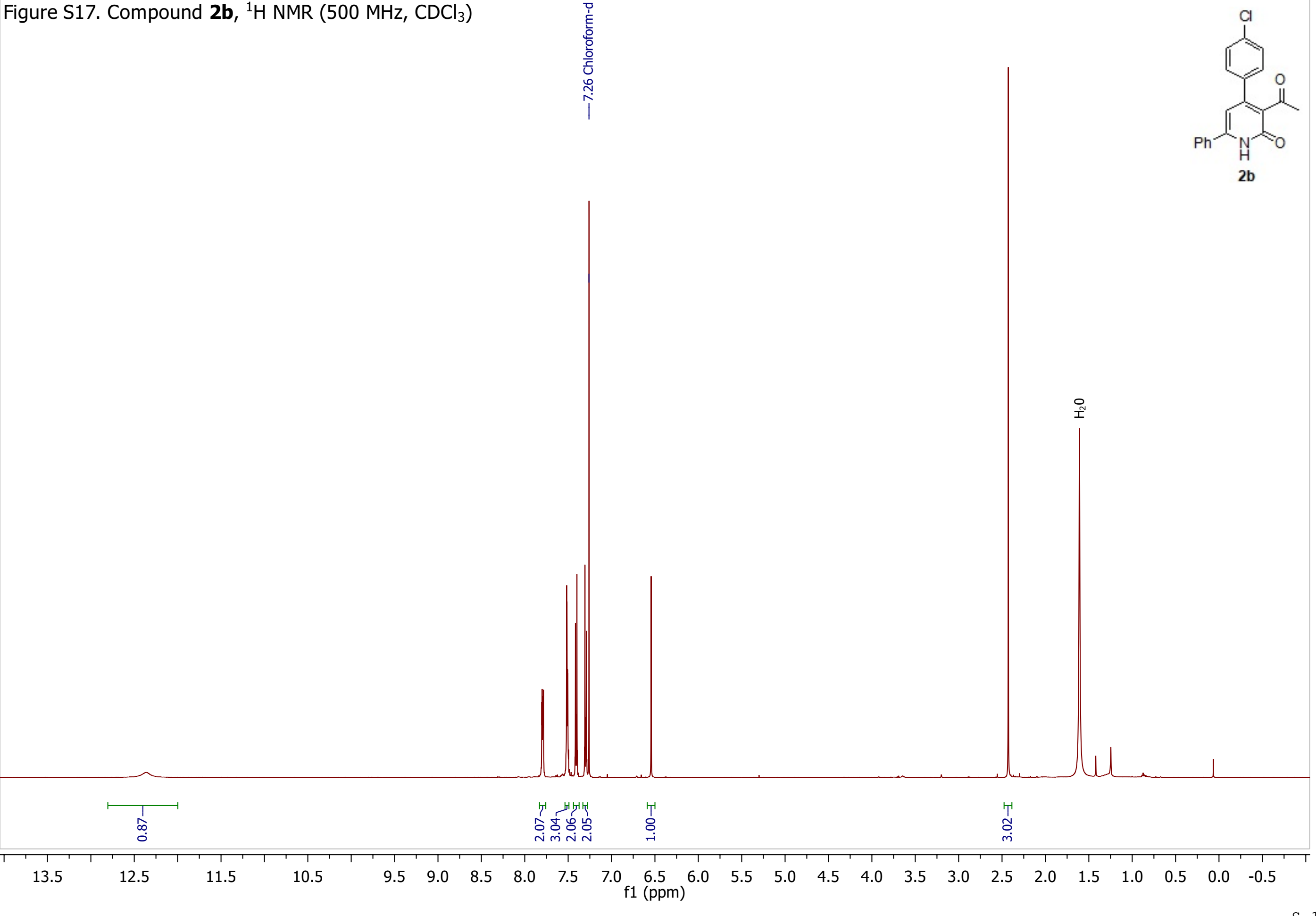

S 18 
Figure S18. Compound $\mathbf{2 b},{ }^{13} \mathrm{C}\left\{{ }^{1} \mathrm{H}\right\}$ NMR $\left(126 \mathrm{MHz}, \mathrm{CDCl}_{3}\right)$

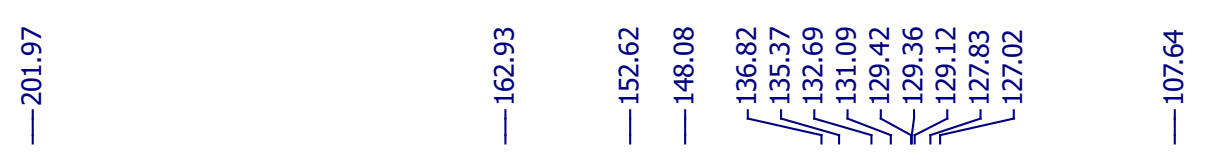

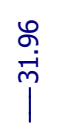

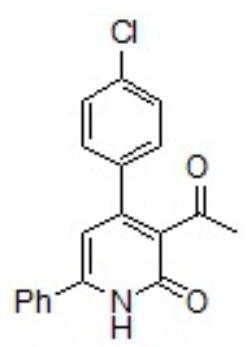

$2 b$

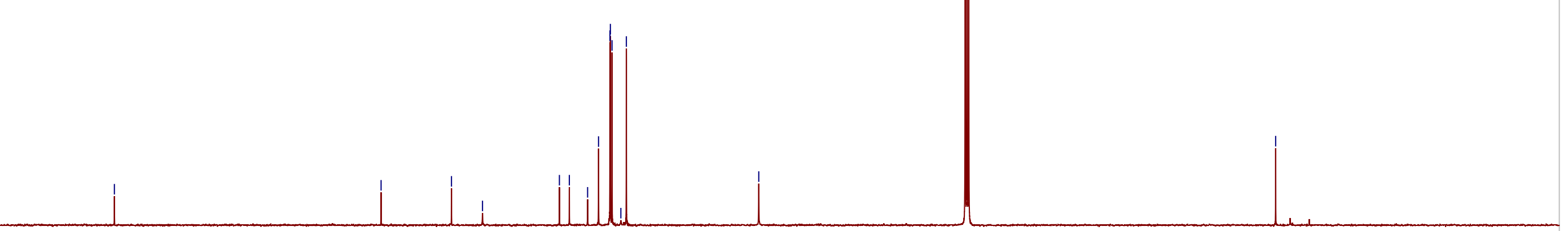




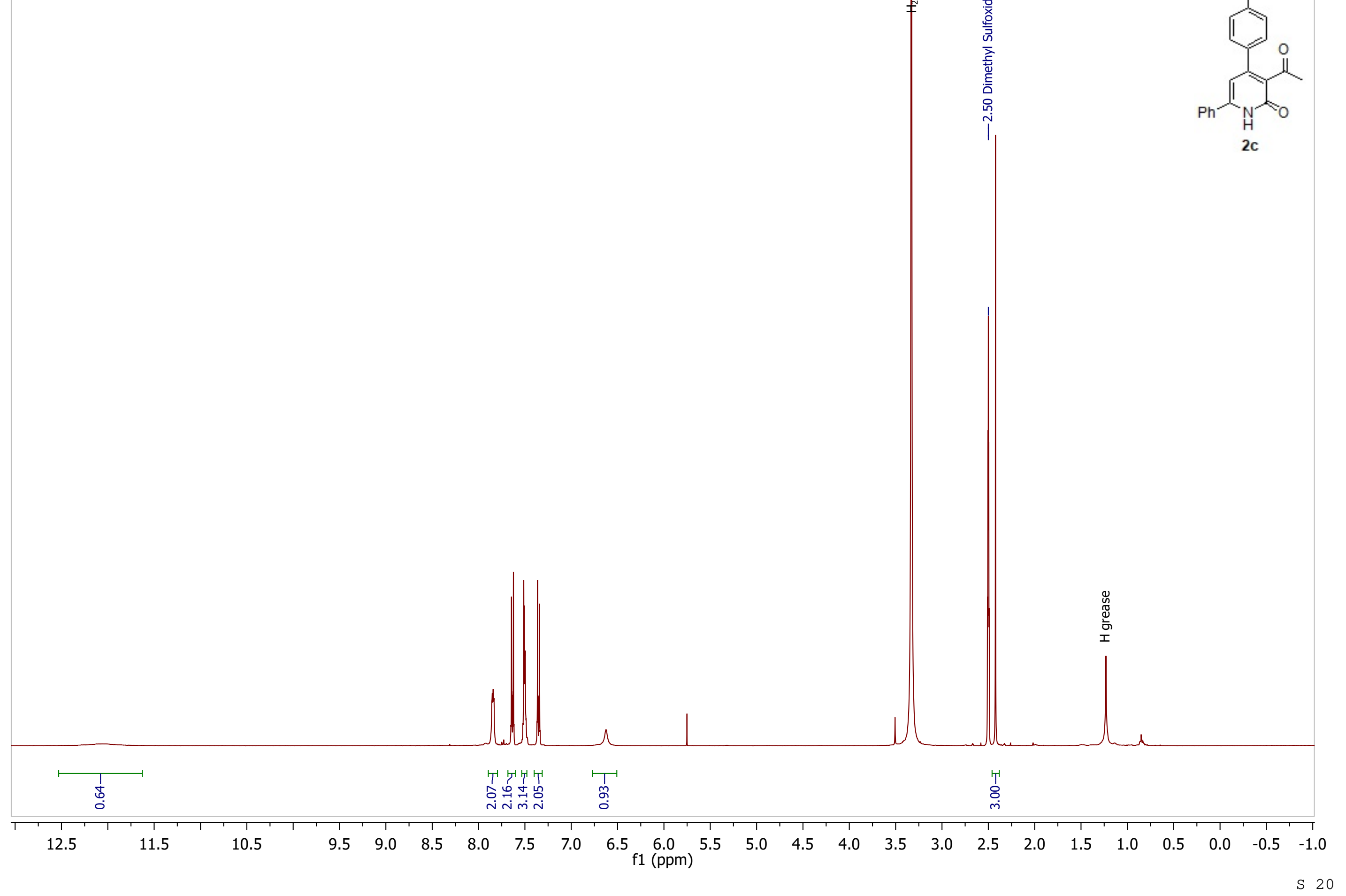


Figure S20. Compound $2 \mathbf{c}^{13} \mathrm{C}\left\{{ }^{1} \mathrm{H}\right\}$ NMR $(126 \mathrm{MHz}$, DMSO-d 6 )
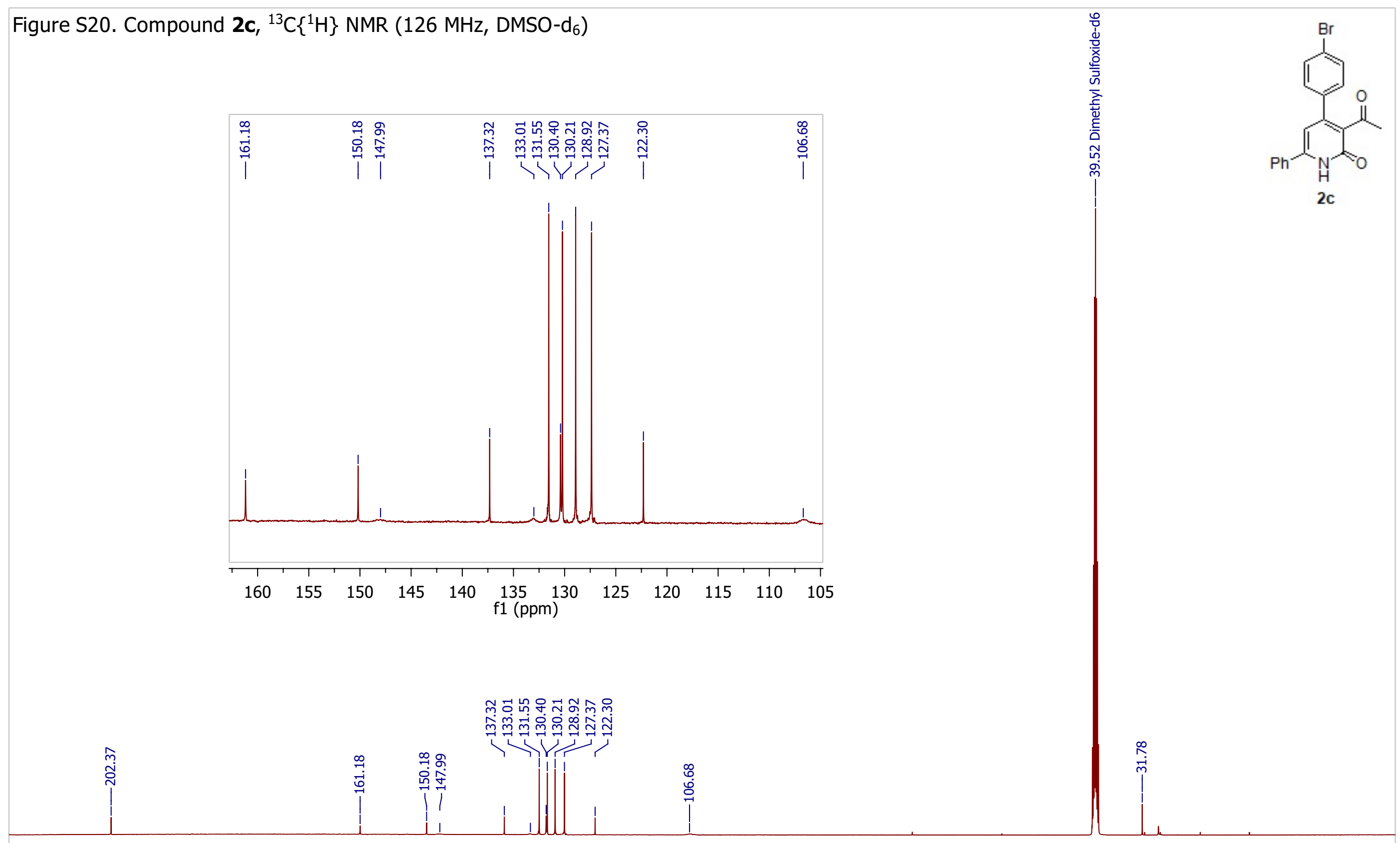

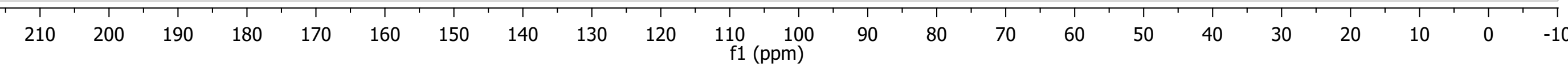


Figure S21. Compound $2 \mathrm{c}$, NOESY ${ }^{1} \mathrm{H}^{-1} \mathrm{H}\left(500 \mathrm{MHz}\right.$, DMSO-d $\left.\mathrm{d}_{6}\right)$
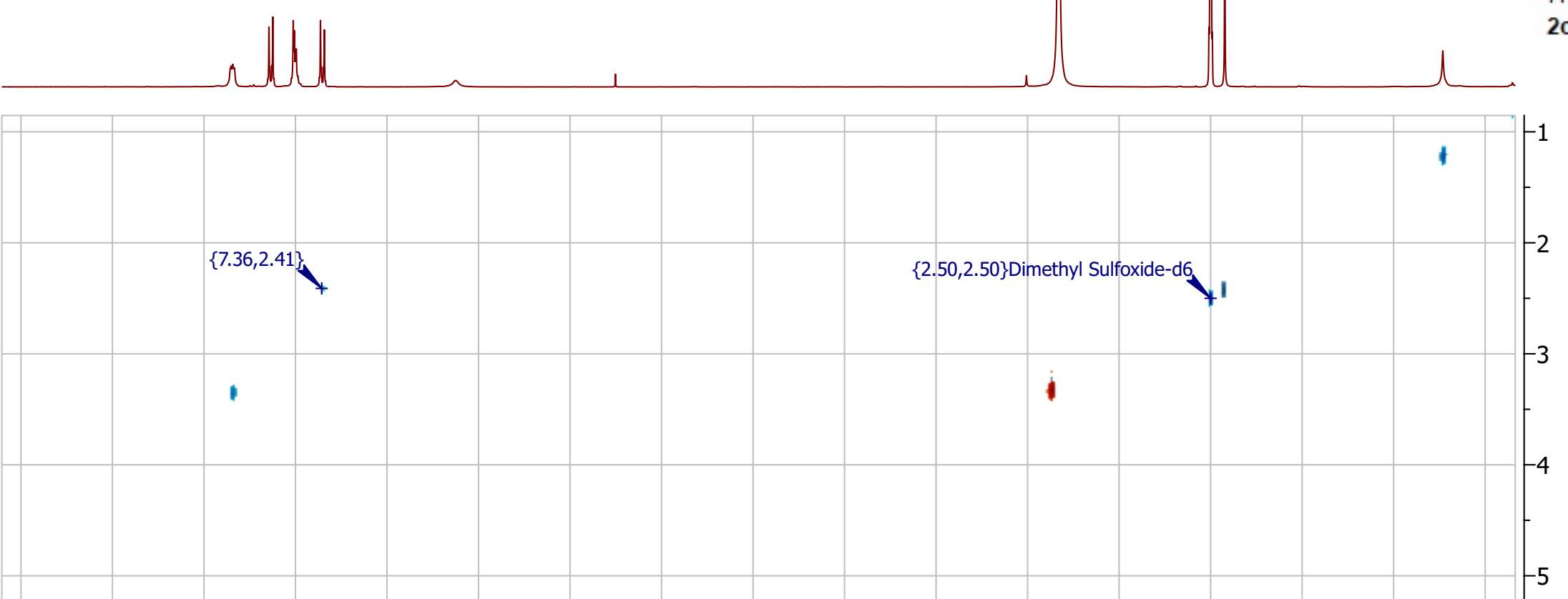

3

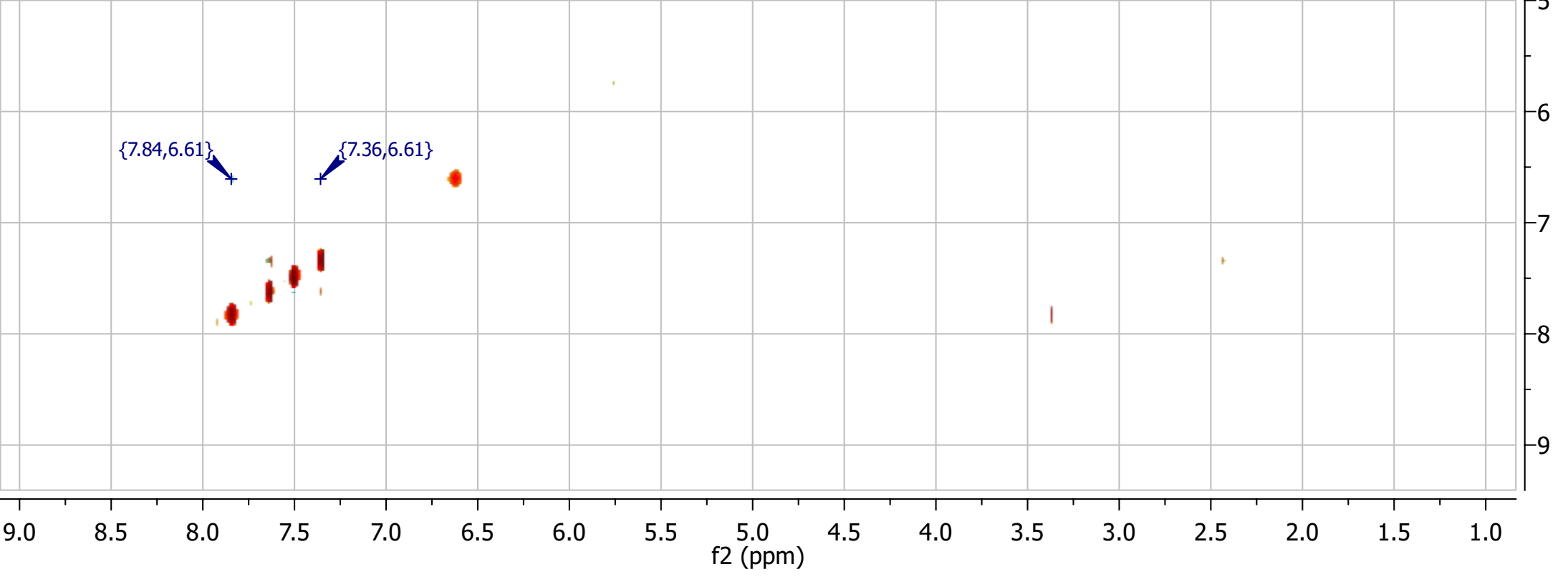

हᄐ 

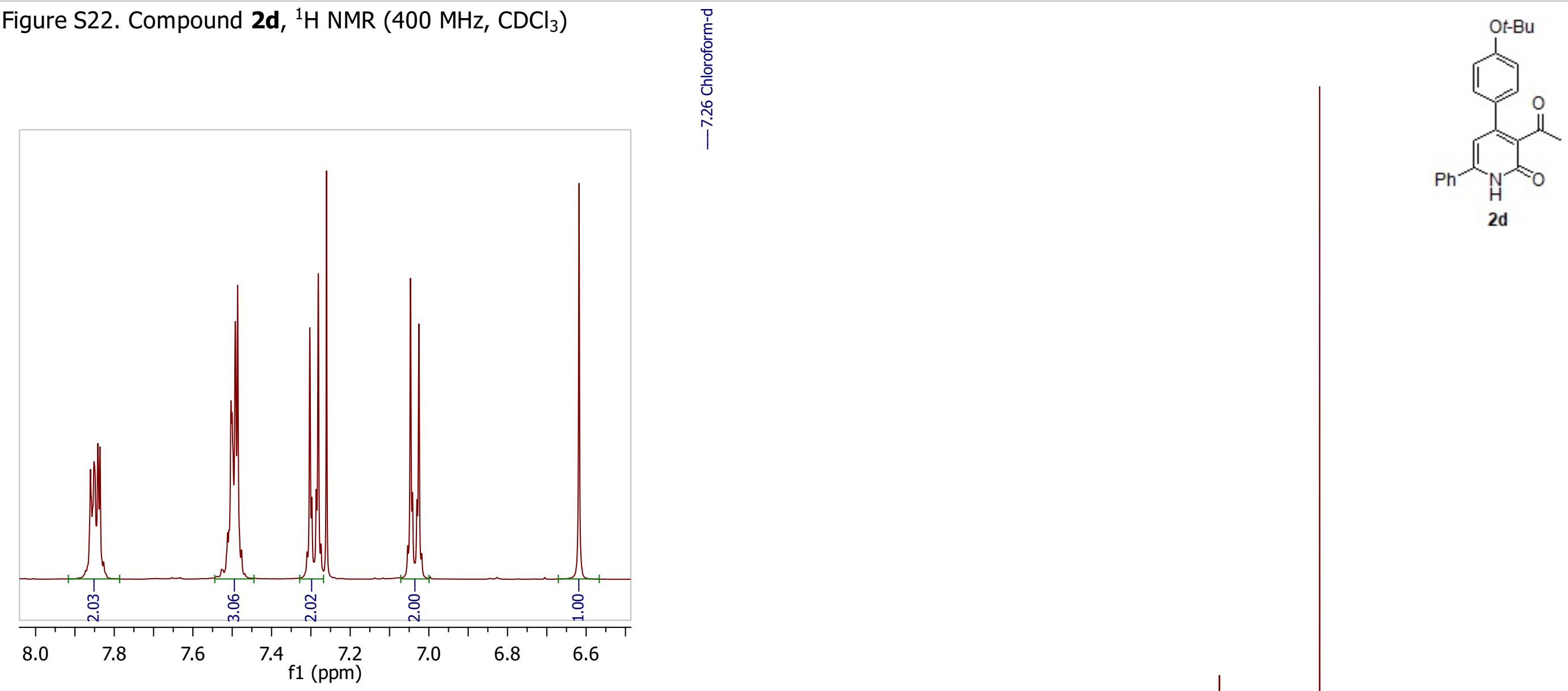

\begin{tabular}{|c|c|c|c|c|c|c|c|c|c|c|c|c|c|c|c|c|c|c|c|c|c|c|c|c|}
\hline & $\begin{array}{l}1 \\
\text { ò } \\
\text { o }\end{array}$ & & & & & & & 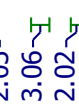 & & $\begin{array}{l}T^{\top} \\
\stackrel{\text { Oे }}{\mathrm{T}}\end{array}$ & & & & & & & & $\begin{array}{l}T \\
\stackrel{T}{1} \\
\stackrel{m}{m}\end{array}$ & & 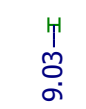 & & & & \\
\hline \begin{tabular}{|l|l|} 
& \\
13.5
\end{tabular} & \begin{tabular}{l|l}
12.5 \\
\end{tabular} & 11.5 & $\begin{array}{l}10.5 \\
10.5\end{array}$ & 9.5 & 9.0 & 8.5 & 8.0 & 7.5 & & $\begin{array}{c}6.5 \\
1(\mathrm{ppm})\end{array}$ & 6.0 & 5.5 & 5.0 & 4.5 & 4.0 & 3.5 & 3.0 & 2.5 & 2.0 & & 1.0 & 0.5 & 0.0 & $\begin{array}{l} \\
-0.5\end{array}$ \\
\hline
\end{tabular}


Figure S23. Compound 2d, ${ }^{13} \mathrm{C}\left\{{ }^{1} \mathrm{H}\right\} \mathrm{NMR}\left(101 \mathrm{MHz}, \mathrm{CDCl}_{3}\right)$

iñ
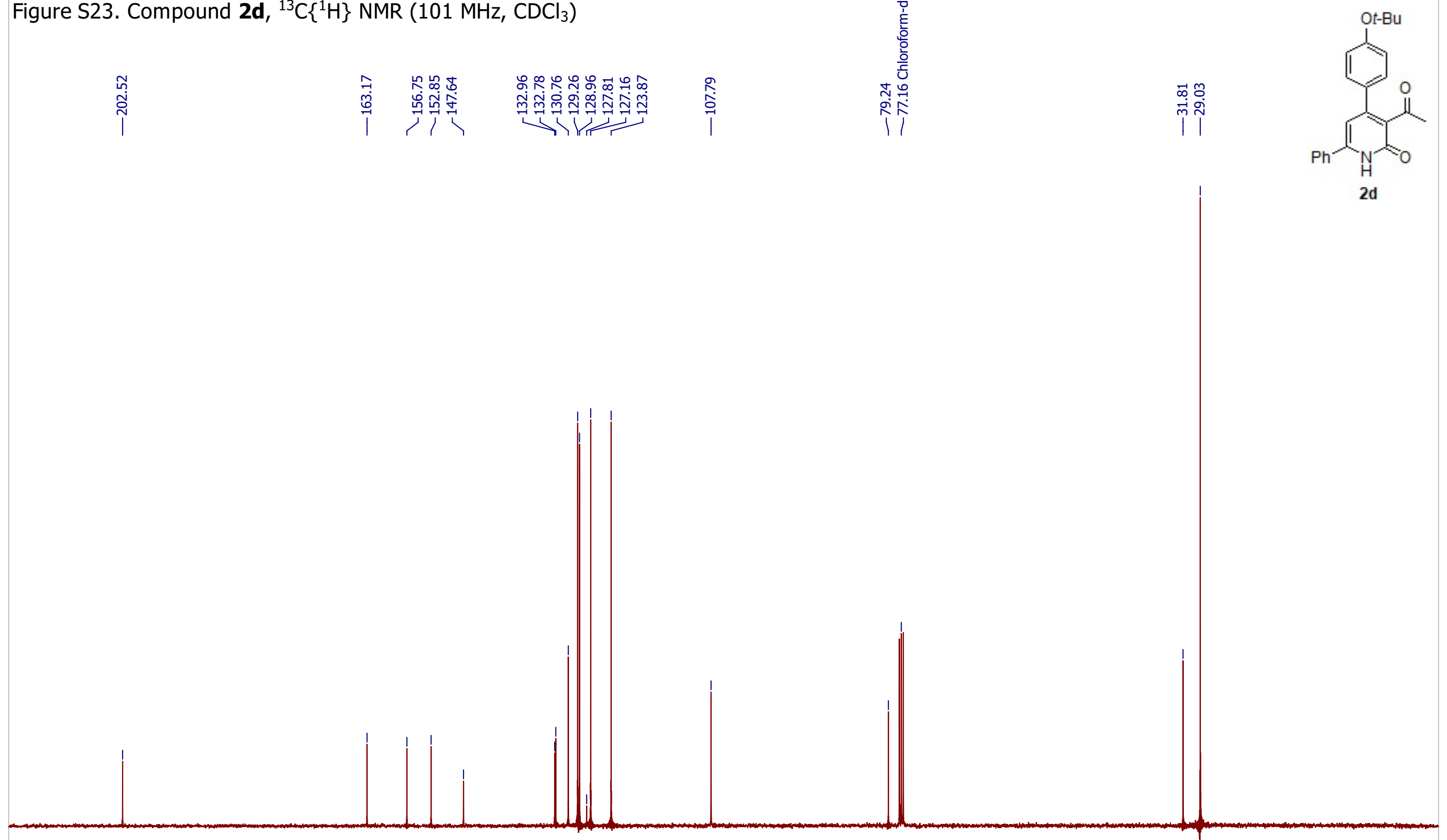

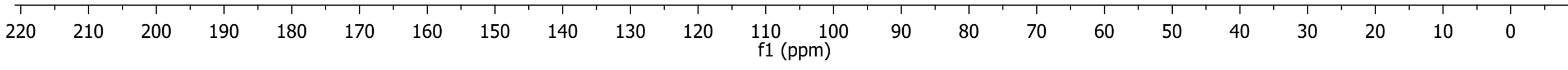


Figure S24. Compound $\mathbf{2 e},{ }^{1} \mathrm{H}$ NMR $\left(400 \mathrm{MHz} \mathrm{CDCl}_{3}\right)$
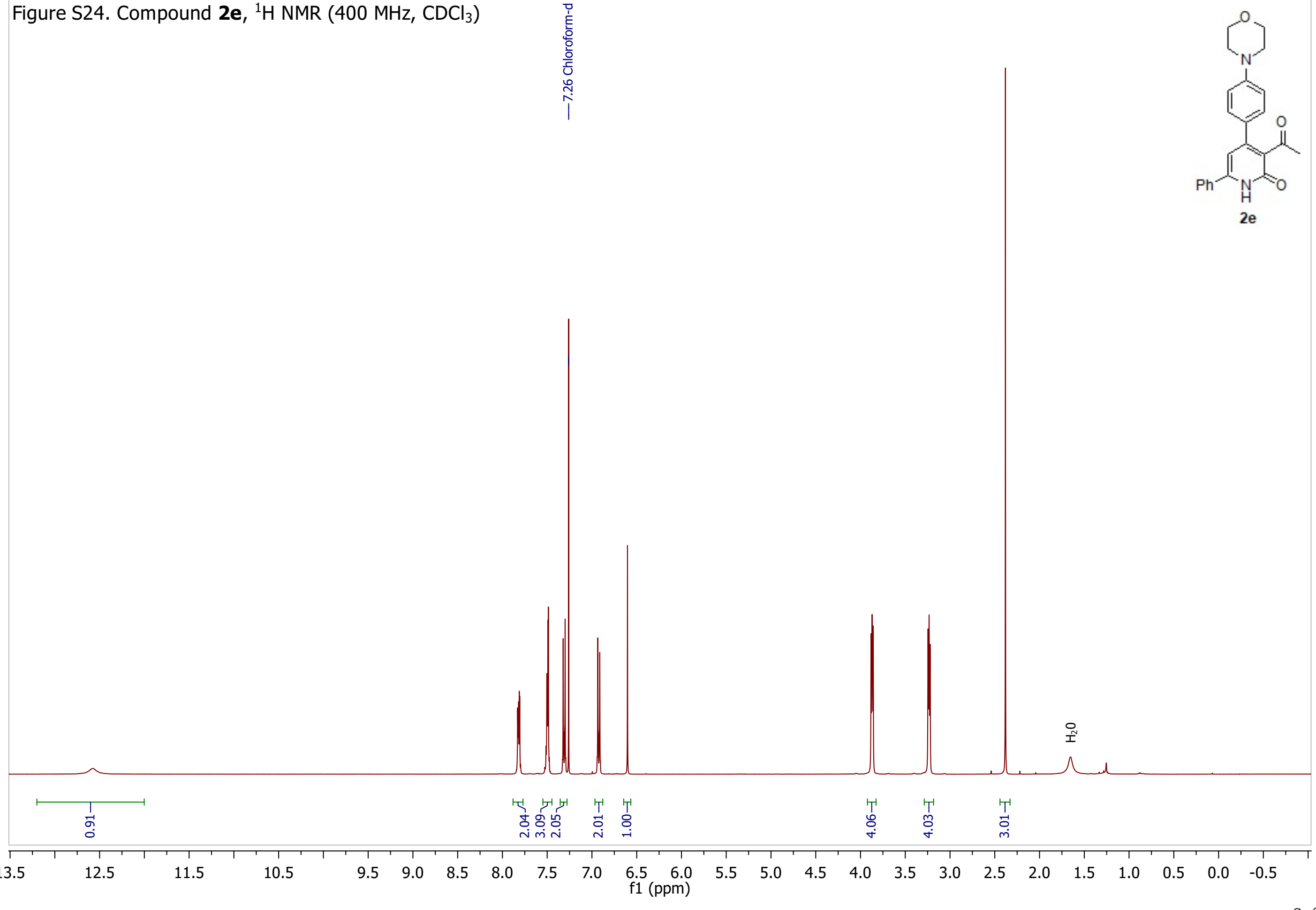

S 25 
Figure S25. Compound $\mathbf{2 e},{ }^{13} \mathrm{C}\left\{{ }^{1} \mathrm{H}\right\}$ NMR $\left(101 \mathrm{MHz}, \mathrm{CDCl}_{3}\right)$

آं
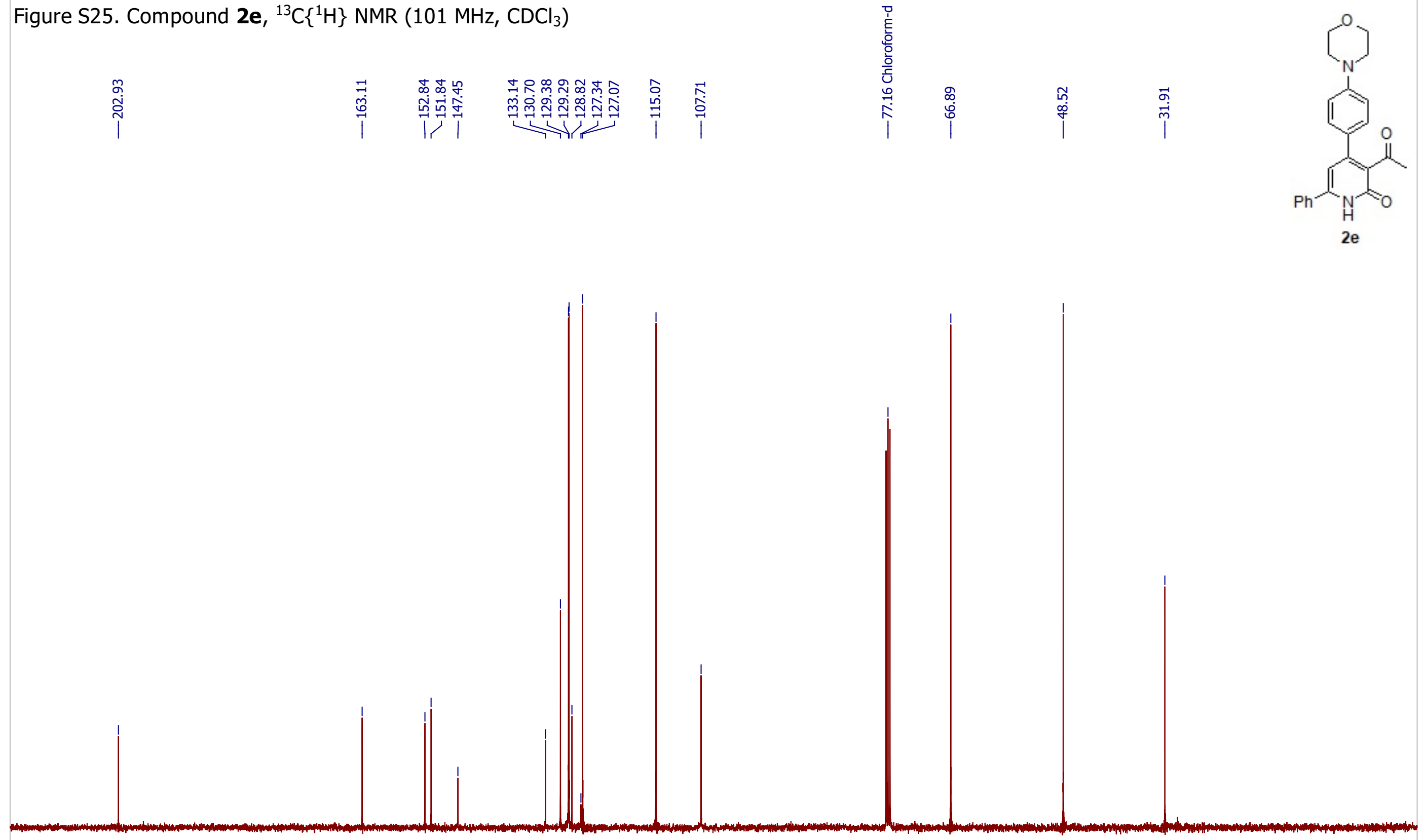

$\begin{array}{rlllllllllllllllllllllll}220 & 210 & 200 & 190 & 180 & 170 & 160 & 150 & 140 & 130 & 120 & \underset{\mathrm{f} 1(\mathrm{ppm})}{110} & 90 & 80 & 70 & 60 & 50 & 40 & 30 & 20 & 10 & 0 & 10\end{array}$




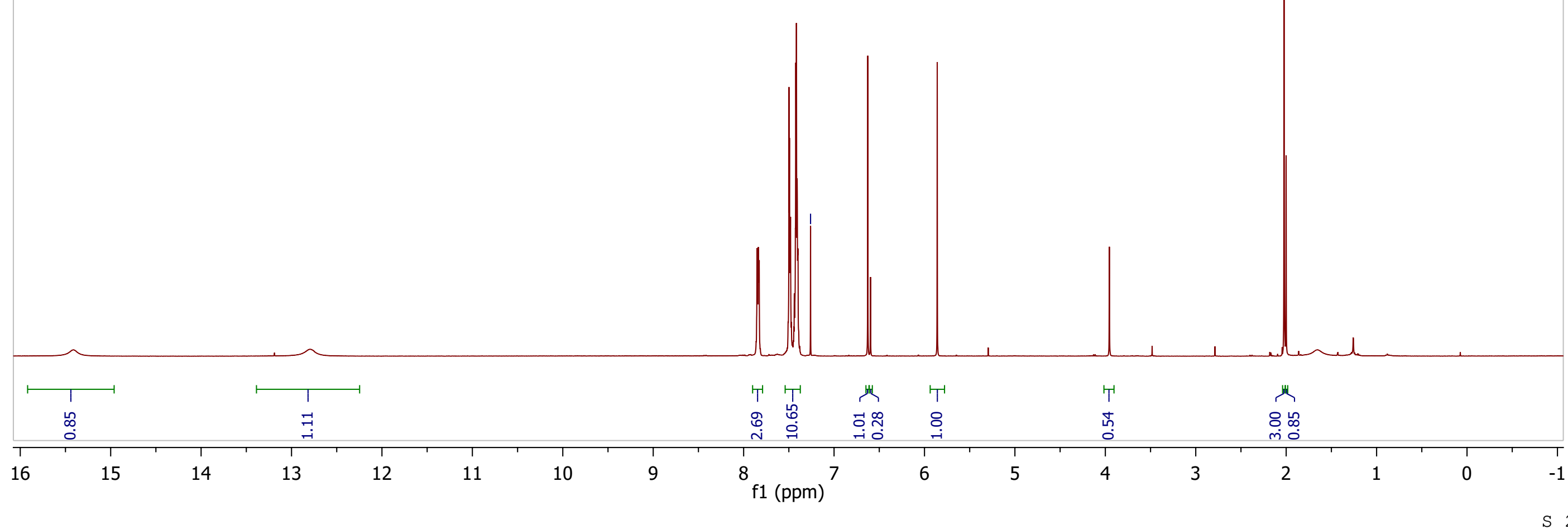


Figure S27. Compound 3a, ${ }^{13} \mathrm{C}\left\{{ }^{1} \mathrm{H}\right\}$ NMR $\left(101 \mathrm{MHz}, \mathrm{CDCl}_{3}\right)$

\begin{tabular}{|c|c|c|c|c|}
\hline 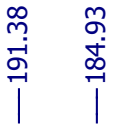 & 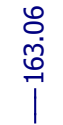 & $\begin{array}{l}\infty \\
\stackrel{\infty}{+} \\
\stackrel{n}{\omega}\end{array}$ & $\underset{\mid}{\stackrel{\infty}{+}}$ & 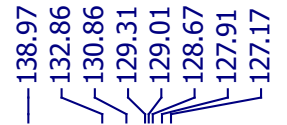 \\
\hline
\end{tabular}
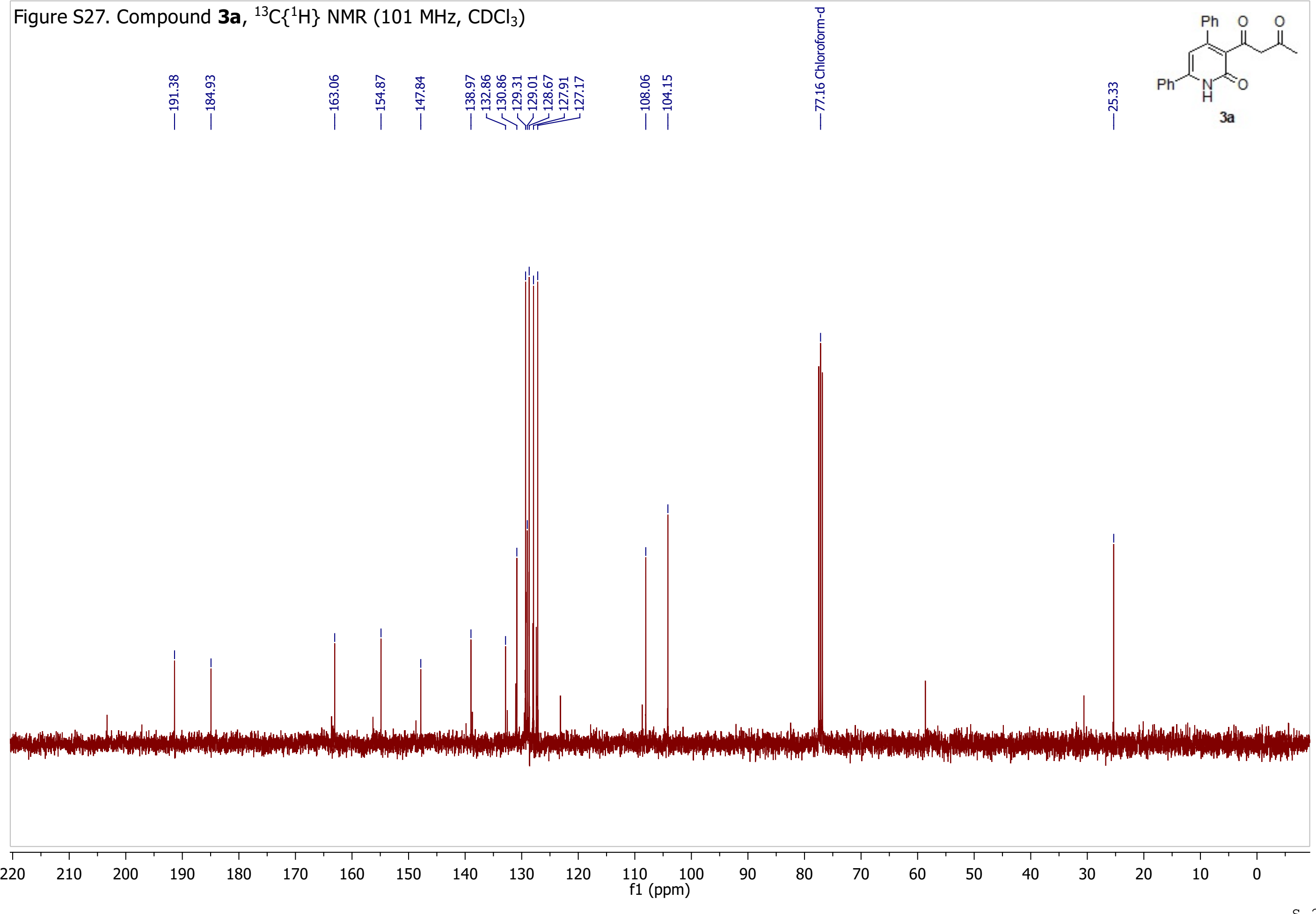
Figure S28. Compound $\mathbf{3 b},{ }^{1} \mathrm{H}$ NMR (400 MHz, $\left.\mathrm{CDCl}_{3}\right)$
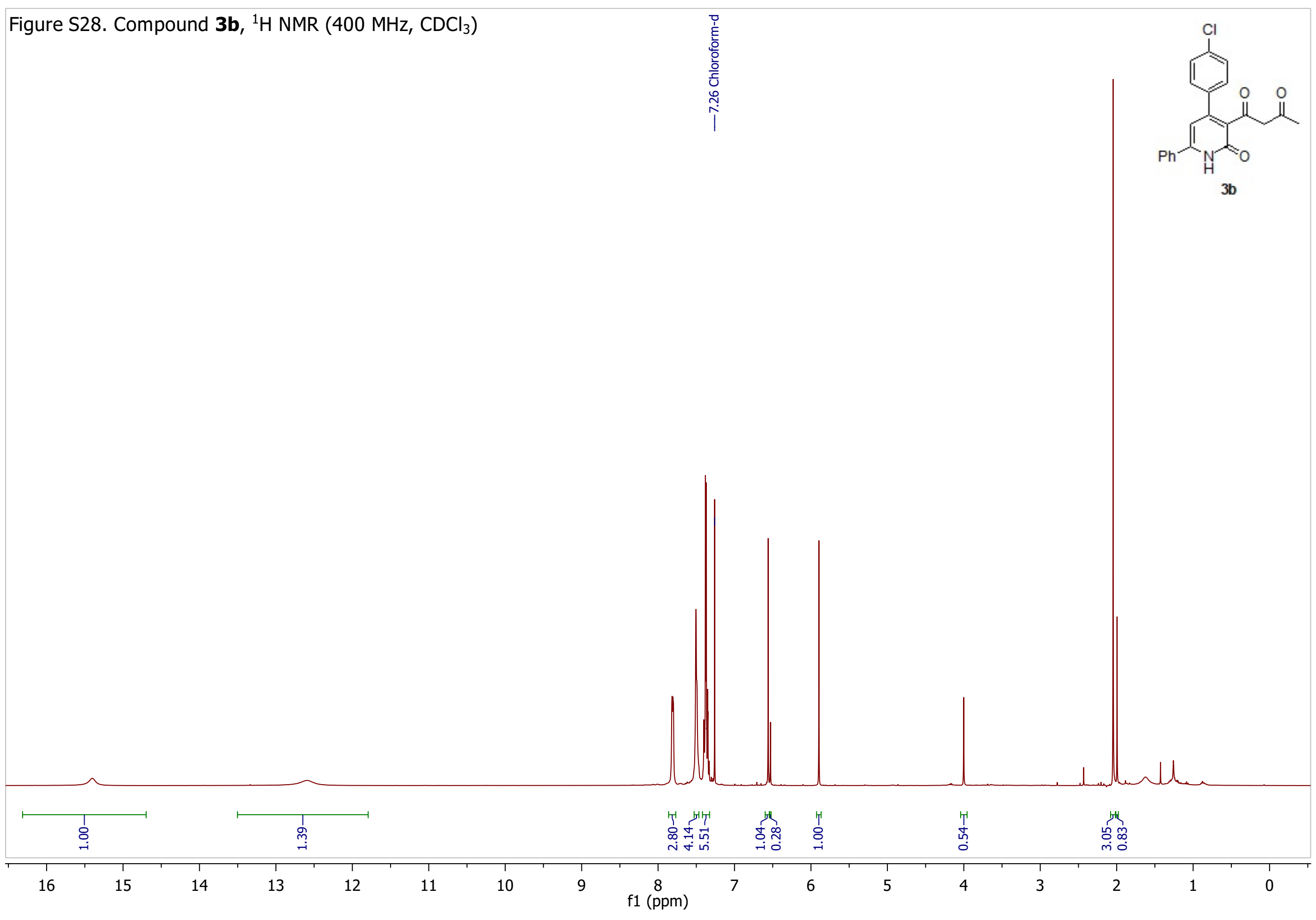


$$
11
$$


Figure S30. Compound 3c, ${ }^{1} \mathrm{H} \mathrm{NMR}\left(400 \mathrm{MHz} \mathrm{CDCl}_{3}\right)$
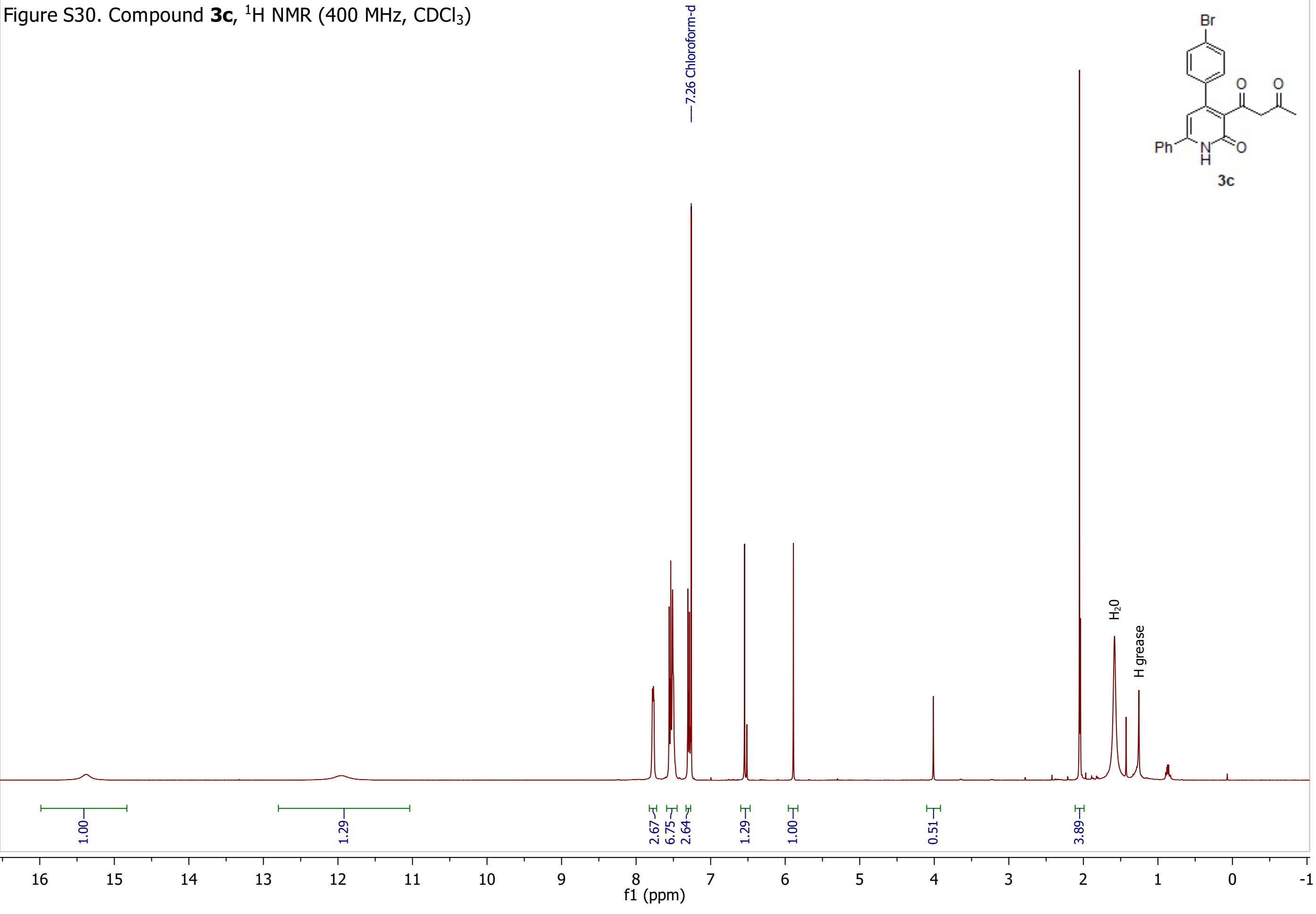
Figure S31. Compound $\mathbf{3 c},{ }^{13} \mathrm{C}\left\{{ }^{1} \mathrm{H}\right\}$ NMR $\left(101 \mathrm{MHz}, \mathrm{CDCl}_{3}\right)$

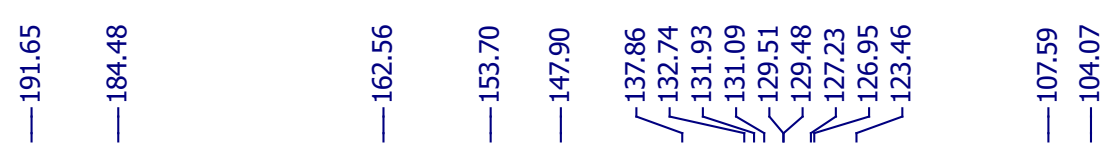
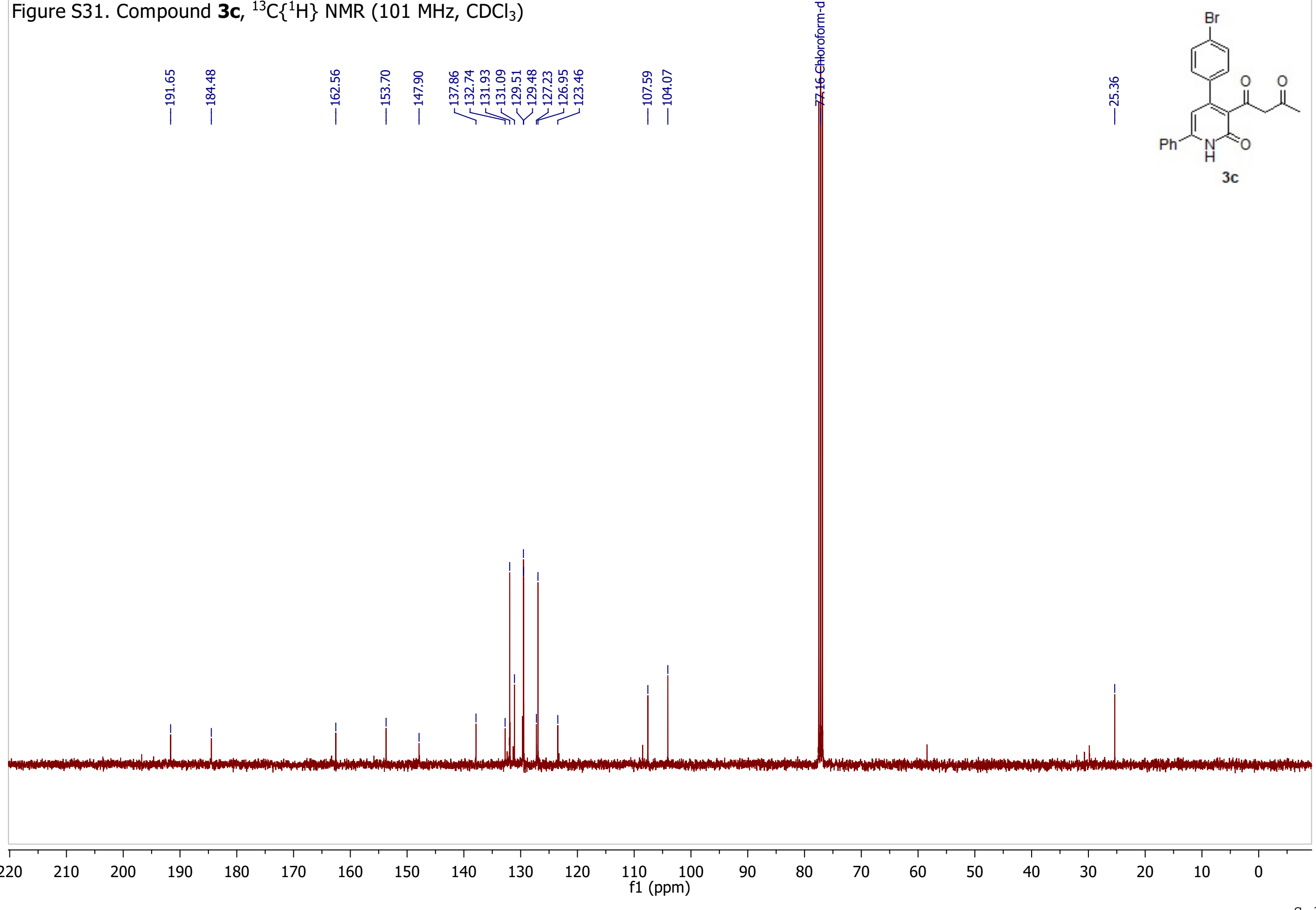

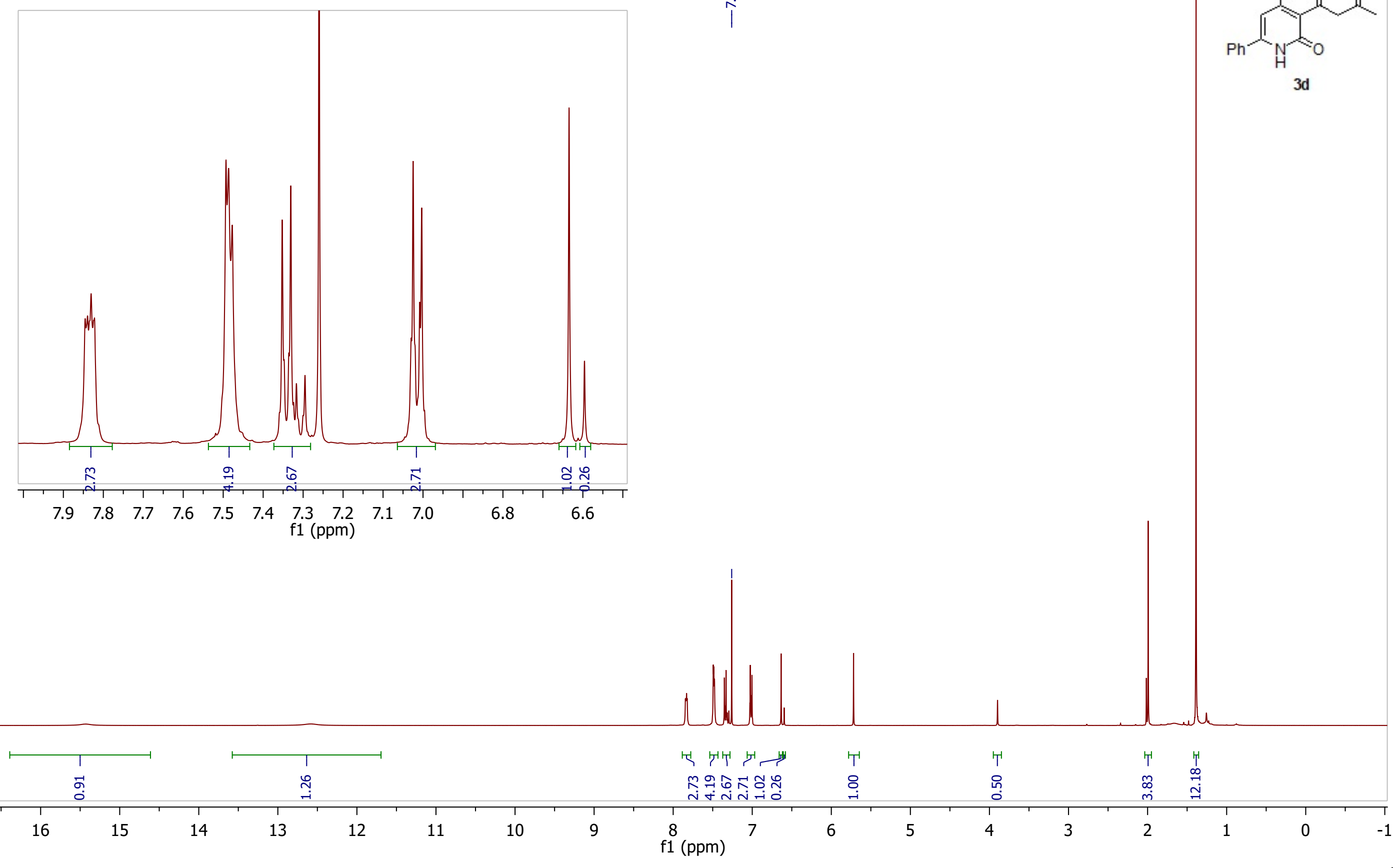
Figure S33. Compound 3d, ${ }^{13} \mathrm{C}\left\{{ }^{1} \mathrm{H}\right\}$ NMR $\left(101 \mathrm{MHz}, \mathrm{CDCl}_{3}\right)$

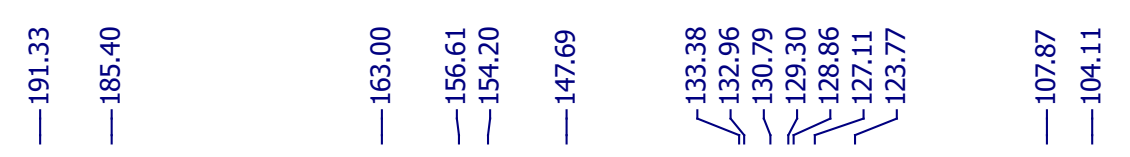


Figure S34. Compound 3e, ${ }^{1} \mathrm{H}$ NMR $\left(400 \mathrm{MHz} \mathrm{CDCl}_{3}\right)$

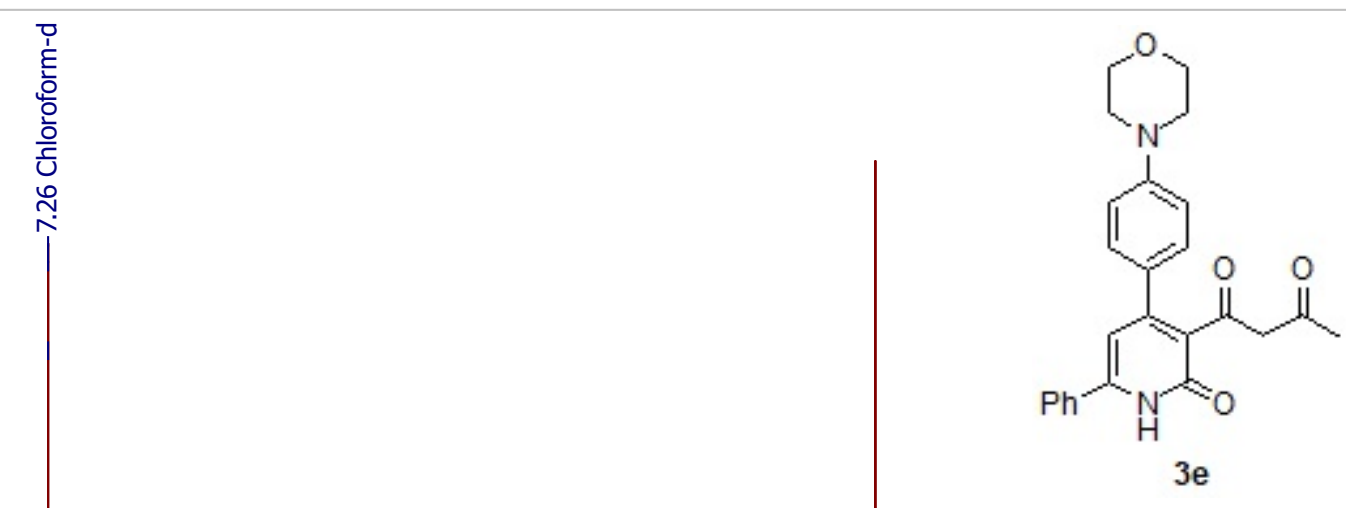

존

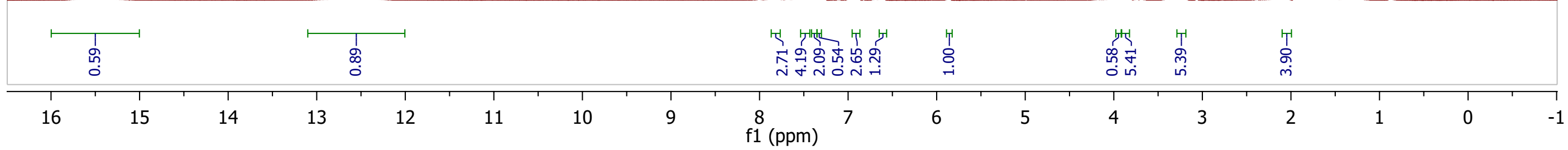


Figure S35. Compound 3e, ${ }^{13} \mathrm{C}\left\{{ }^{1} \mathrm{H}\right\}$ NMR $\left(101 \mathrm{MHz}, \mathrm{CDCl}_{3}\right)$

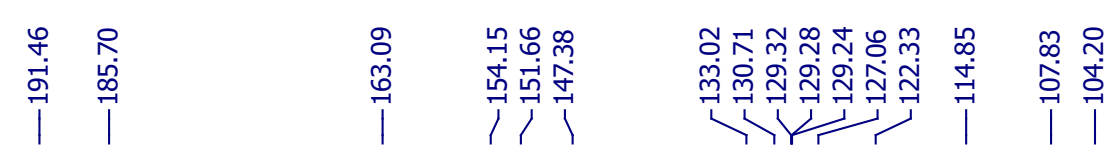
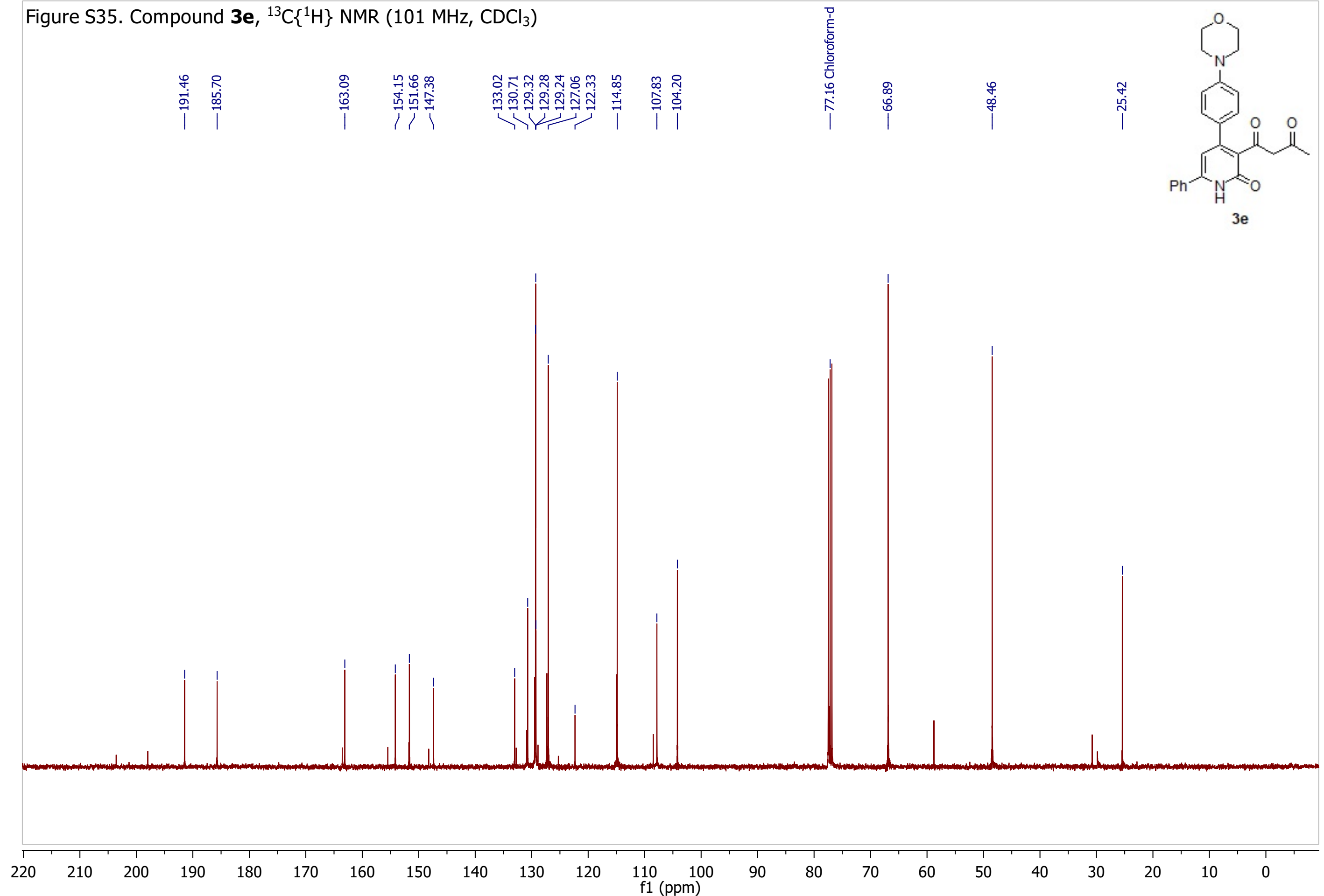


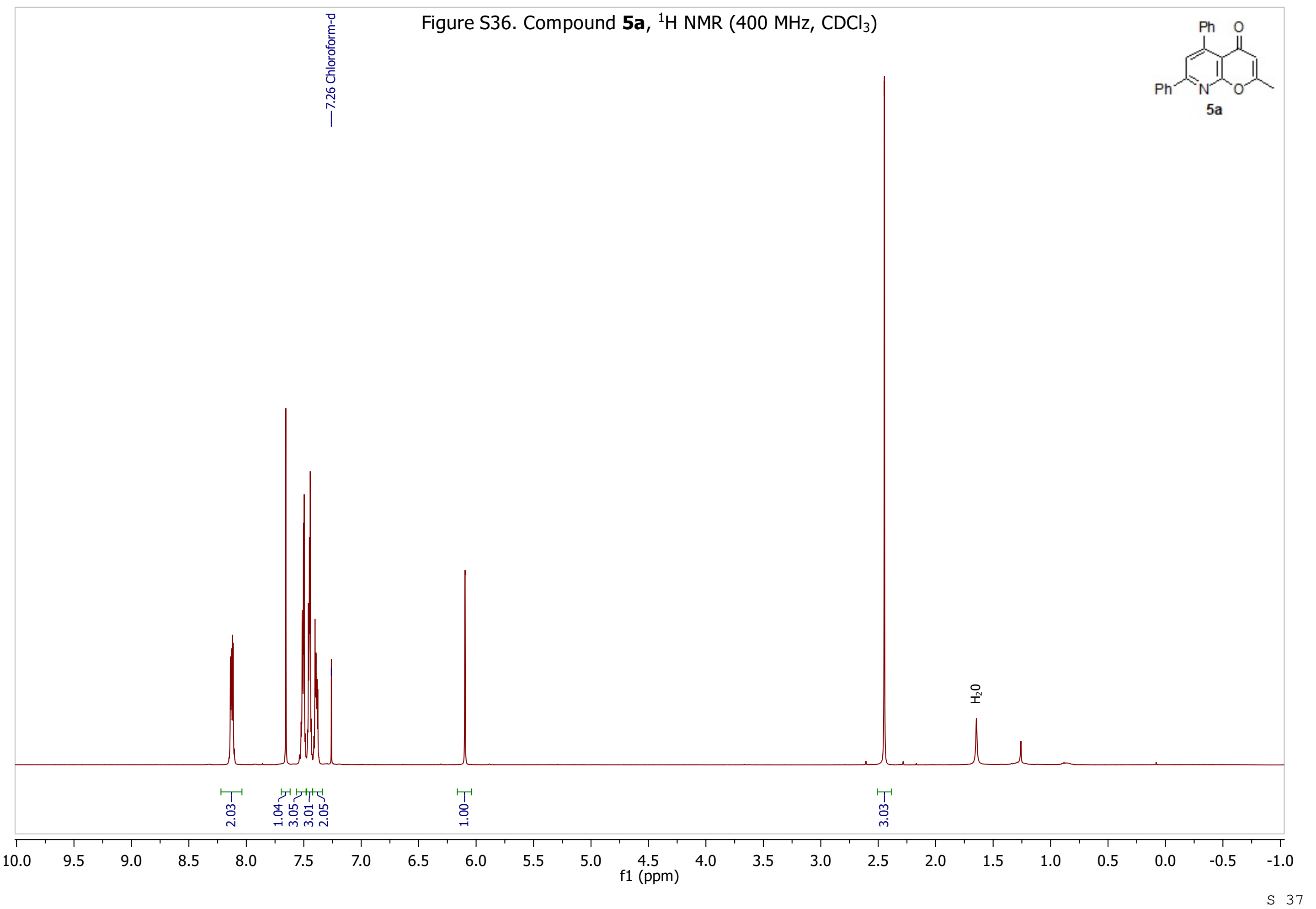


Figure S37. Compound 5a, ${ }^{13} \mathrm{C}\left\{{ }^{1} \mathrm{H}\right\}$ NMR $\left(101 \mathrm{MHz}, \mathrm{CDCl}_{3}\right)$

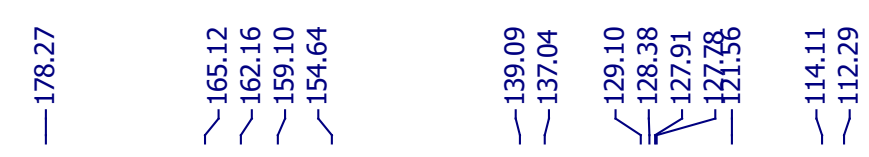

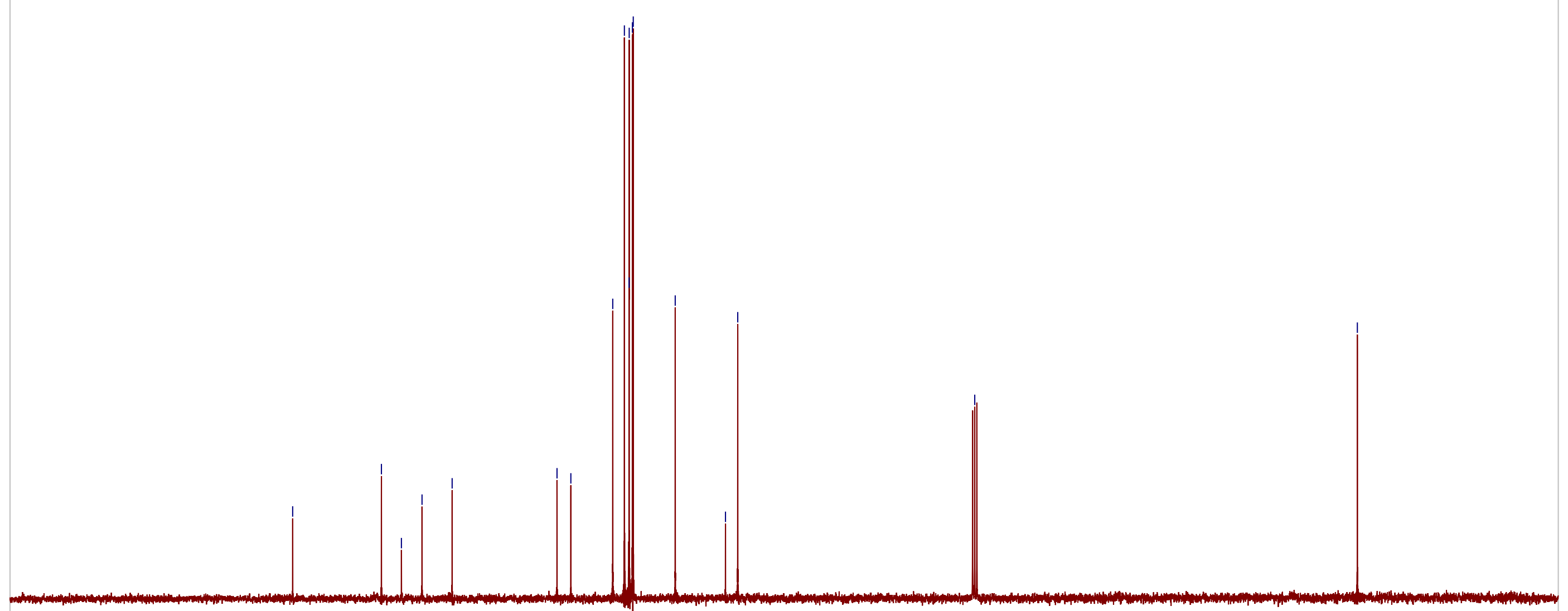

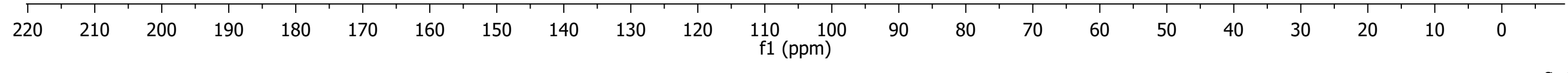




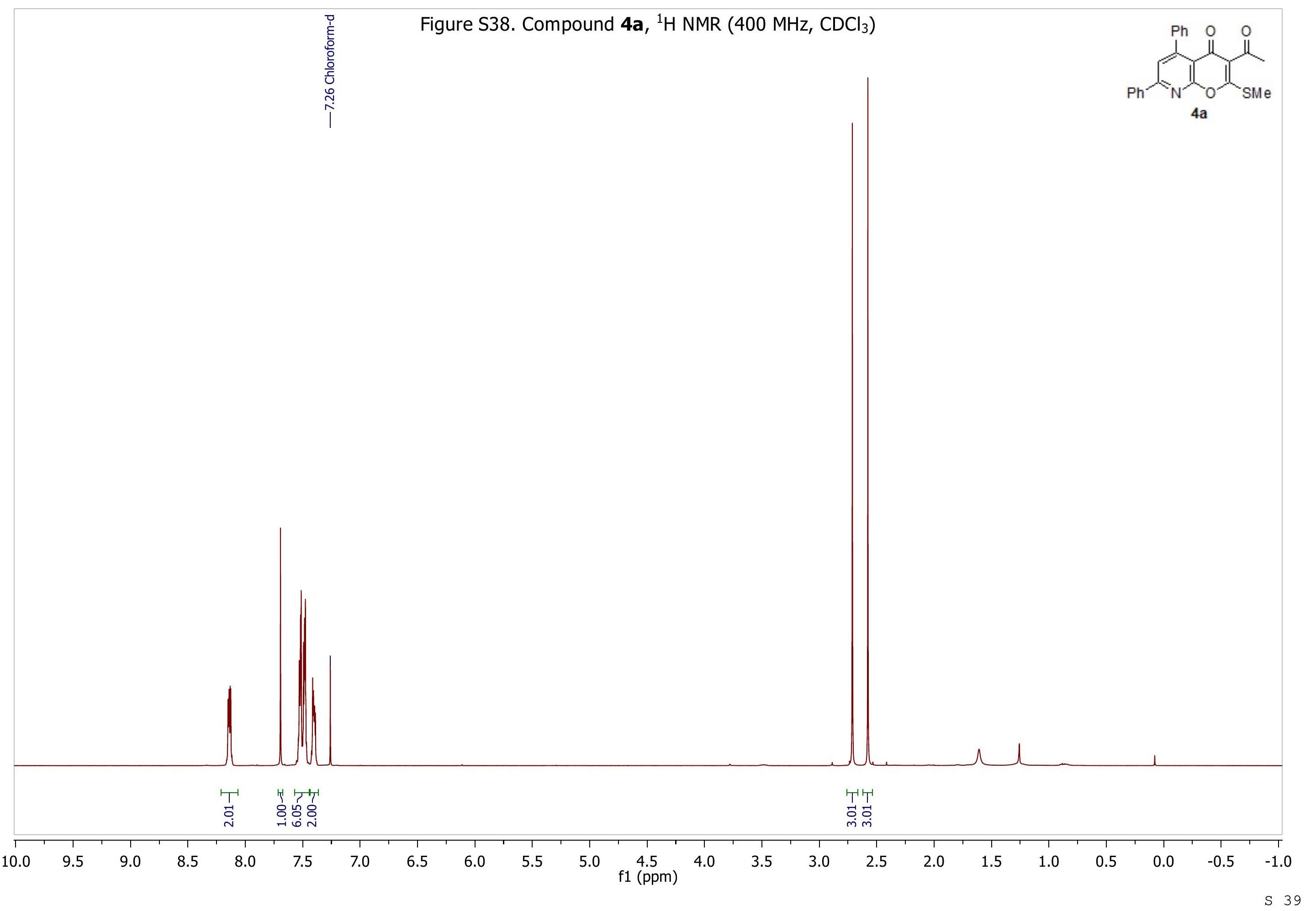


Figure S39. Compound $\mathbf{4 a},{ }^{13} \mathrm{C}\left\{{ }^{1} \mathrm{H}\right\}$ NMR $\left(101 \mathrm{MHz}, \mathrm{CDCl}_{3}\right)$

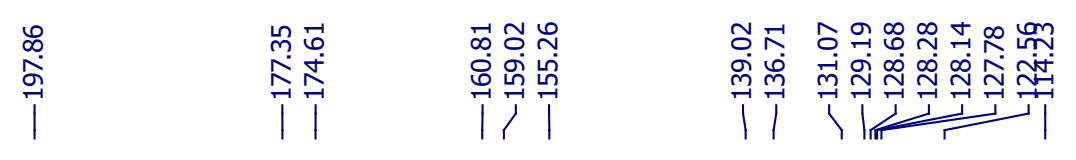

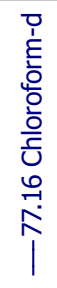<smiles>CC(=O)c1c(C)oc2nc(-c3ccccc3)cc(-c3ccccc3)c2c1=O</smiles>

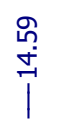

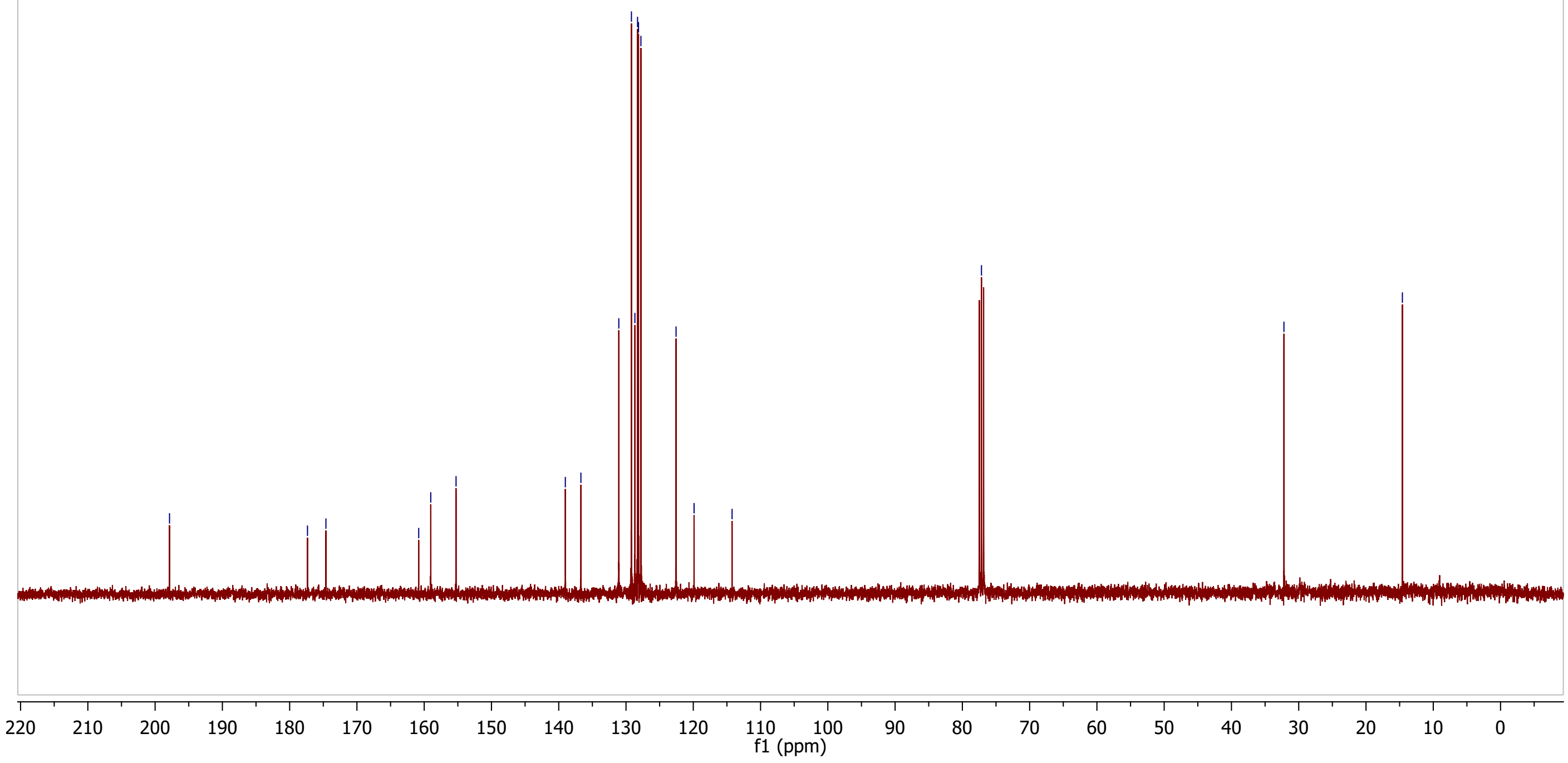

S 40 
Figure S40. Compound 4a, ${ }^{1} \mathrm{H}-{ }^{13} \mathrm{C}$ HSQC (400 MHz, $\left.\mathrm{CDCl}_{3}\right)$
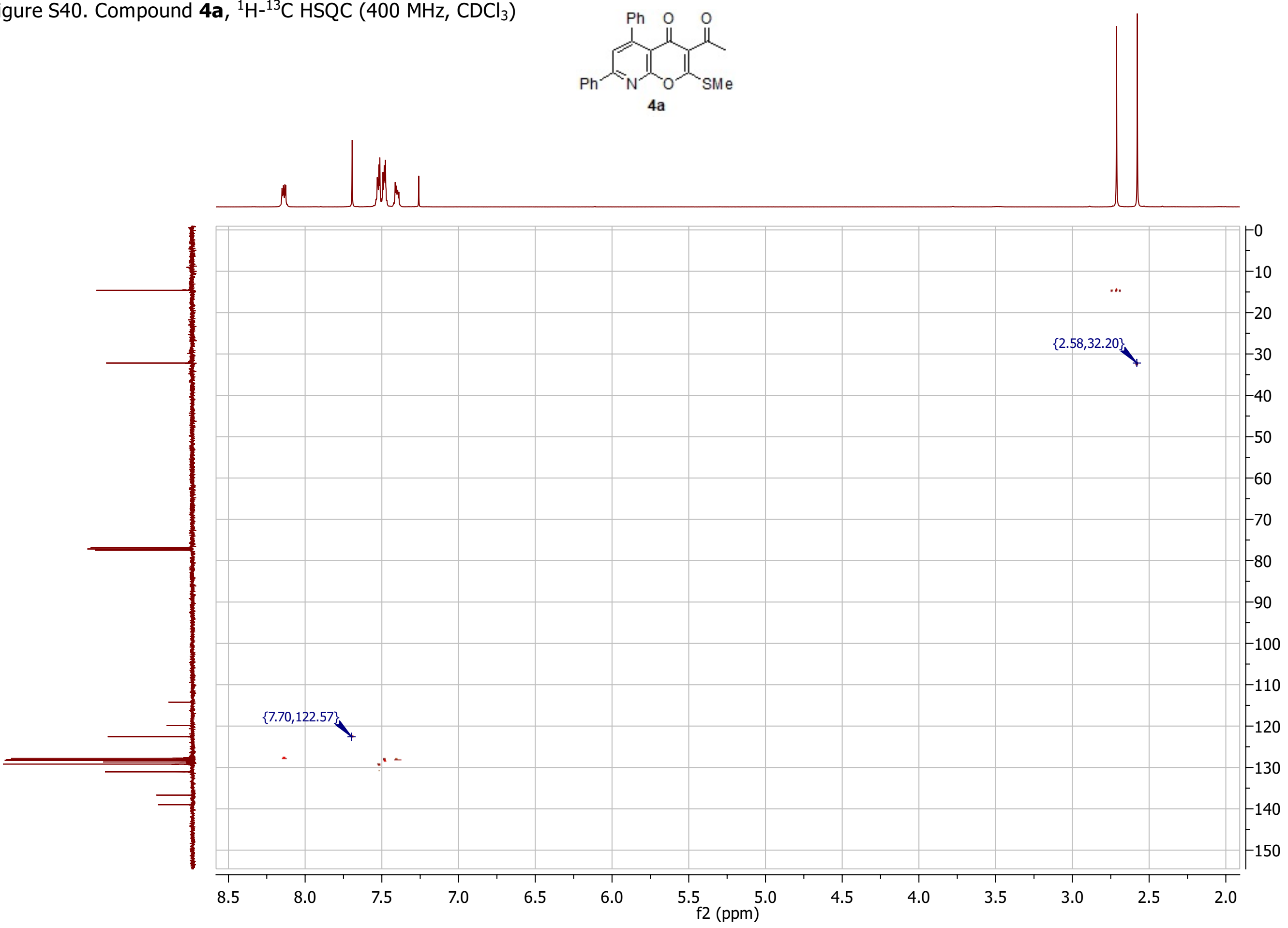

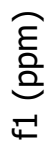


Figure S41. Compound $\mathbf{4 a},{ }^{1} \mathrm{H}^{13} \mathrm{C} \mathrm{HMBC}\left(400 \mathrm{MHz}, \mathrm{CDCl}_{3}\right)$
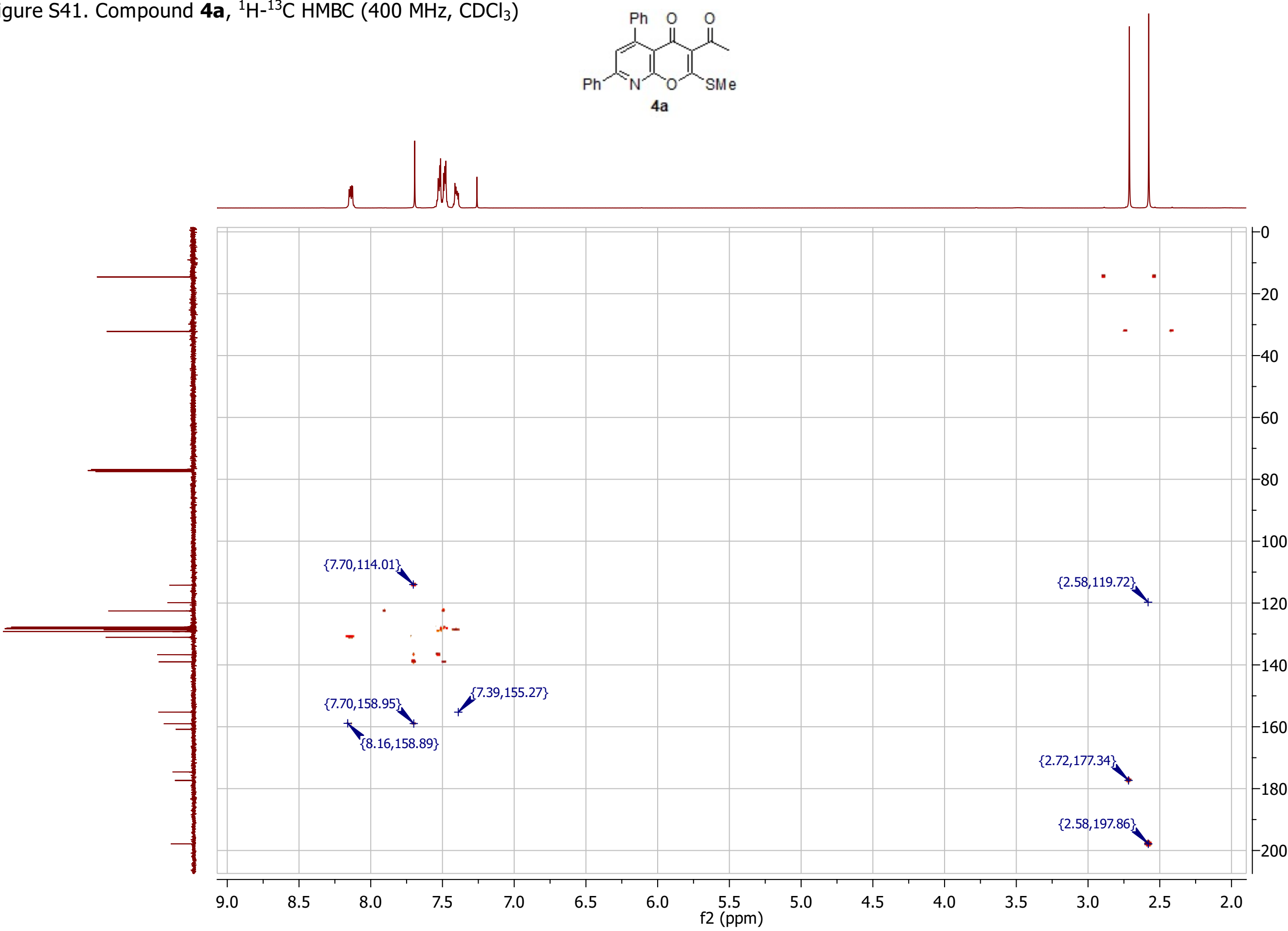

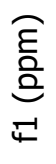
20 


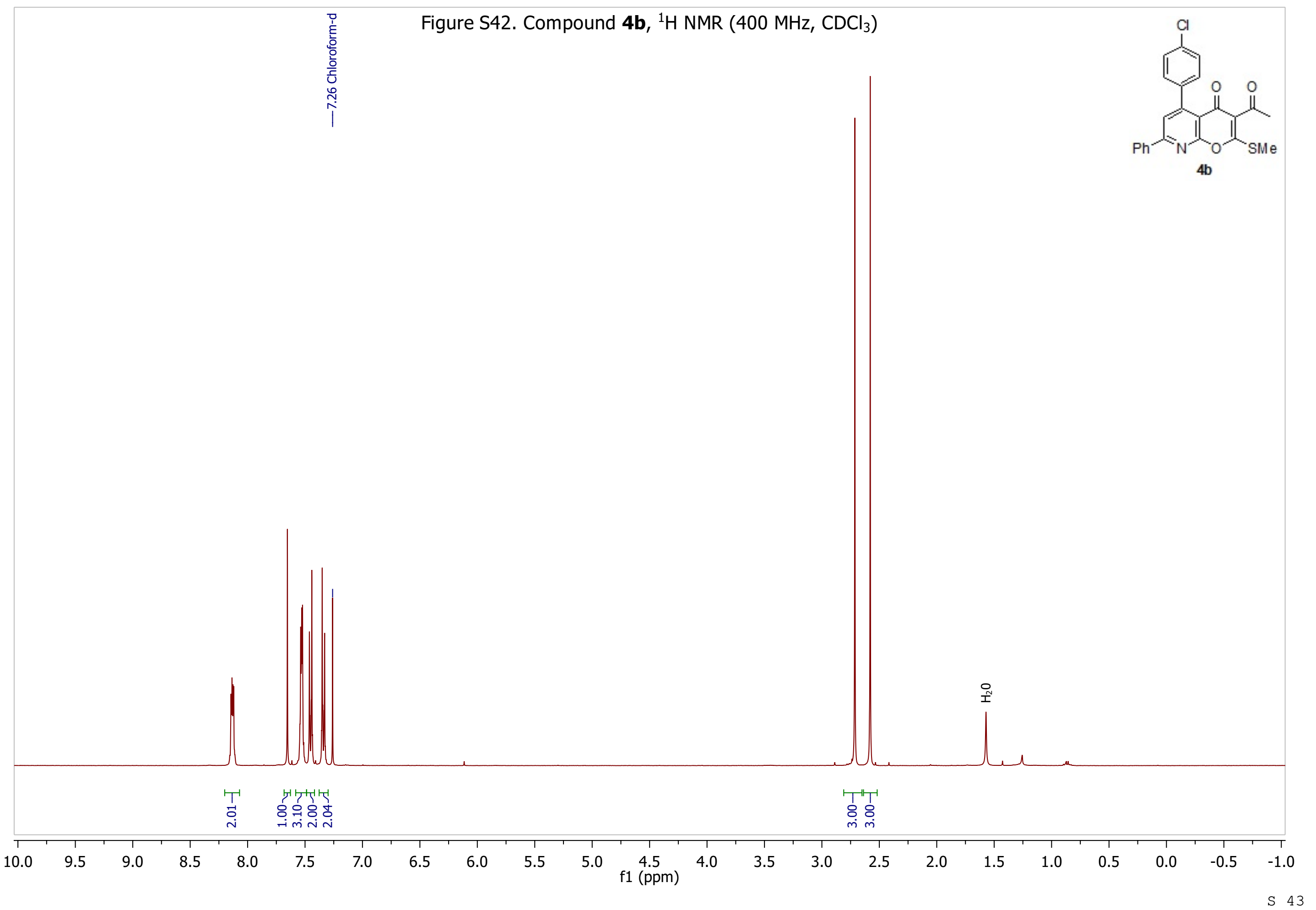


Figure S43. Compound $\mathbf{4 b},{ }^{13} \mathrm{C}\left\{{ }^{1} \mathrm{H}\right\} \mathrm{NMR}\left(101 \mathrm{MHz}, \mathrm{CDCl}_{3}\right)$

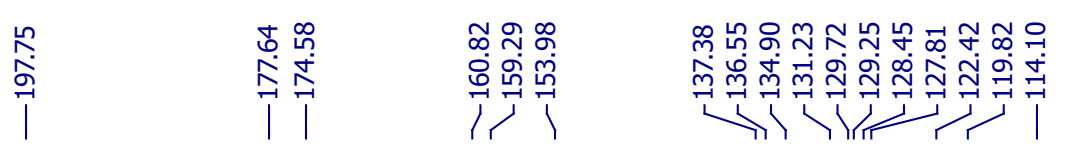

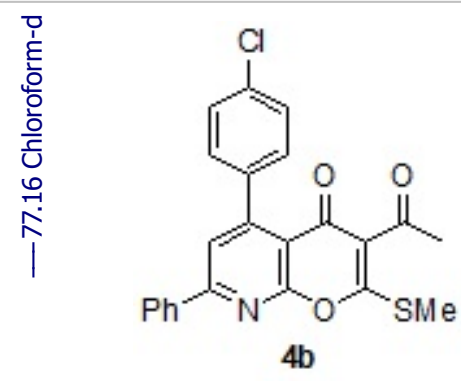

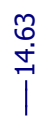

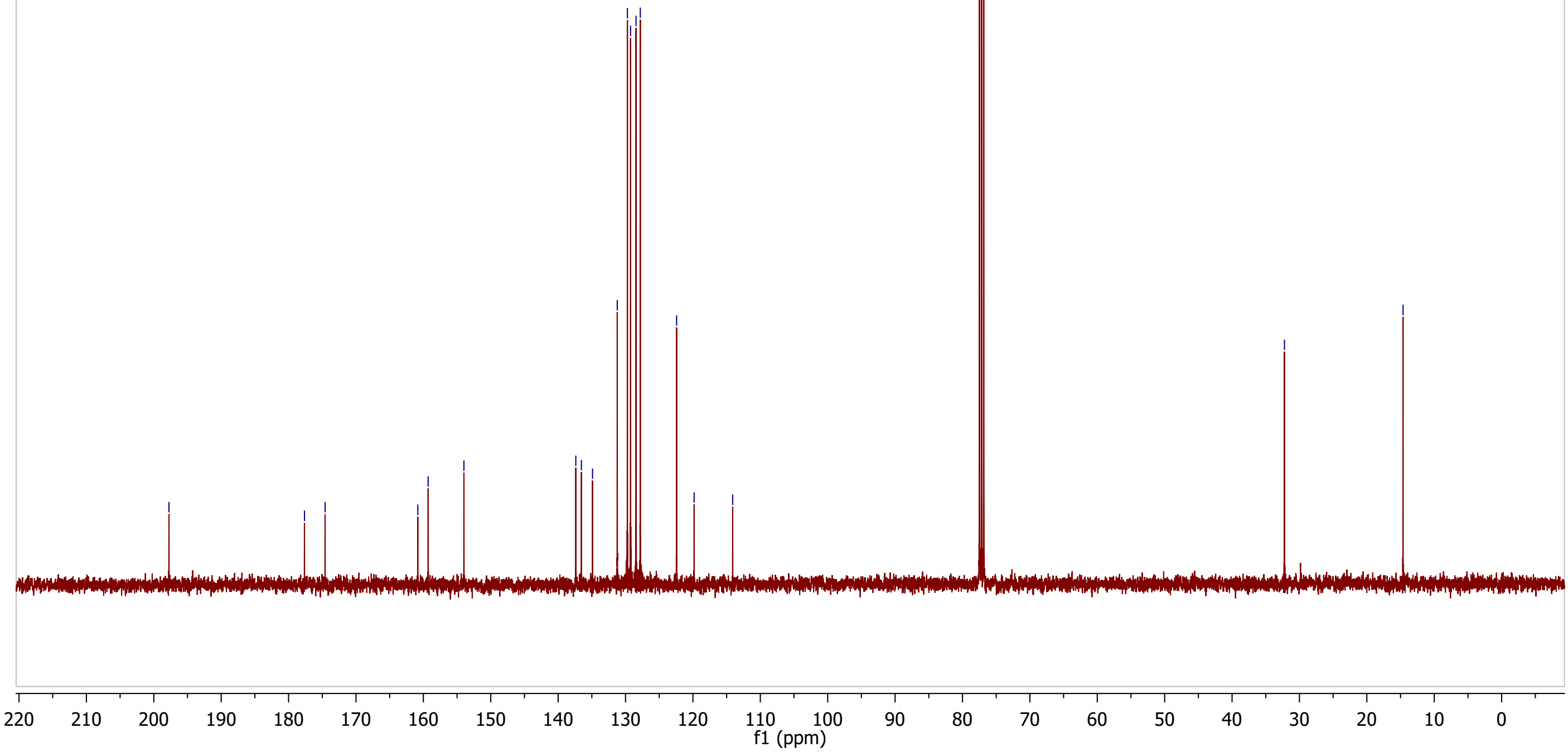

S 44 
Figure S44. Compound 4b, ${ }^{1} \mathrm{H}_{-}{ }^{13} \mathrm{C} \mathrm{HMBC}\left(400 \mathrm{MHz}, \mathrm{CDCl}_{3}\right)$

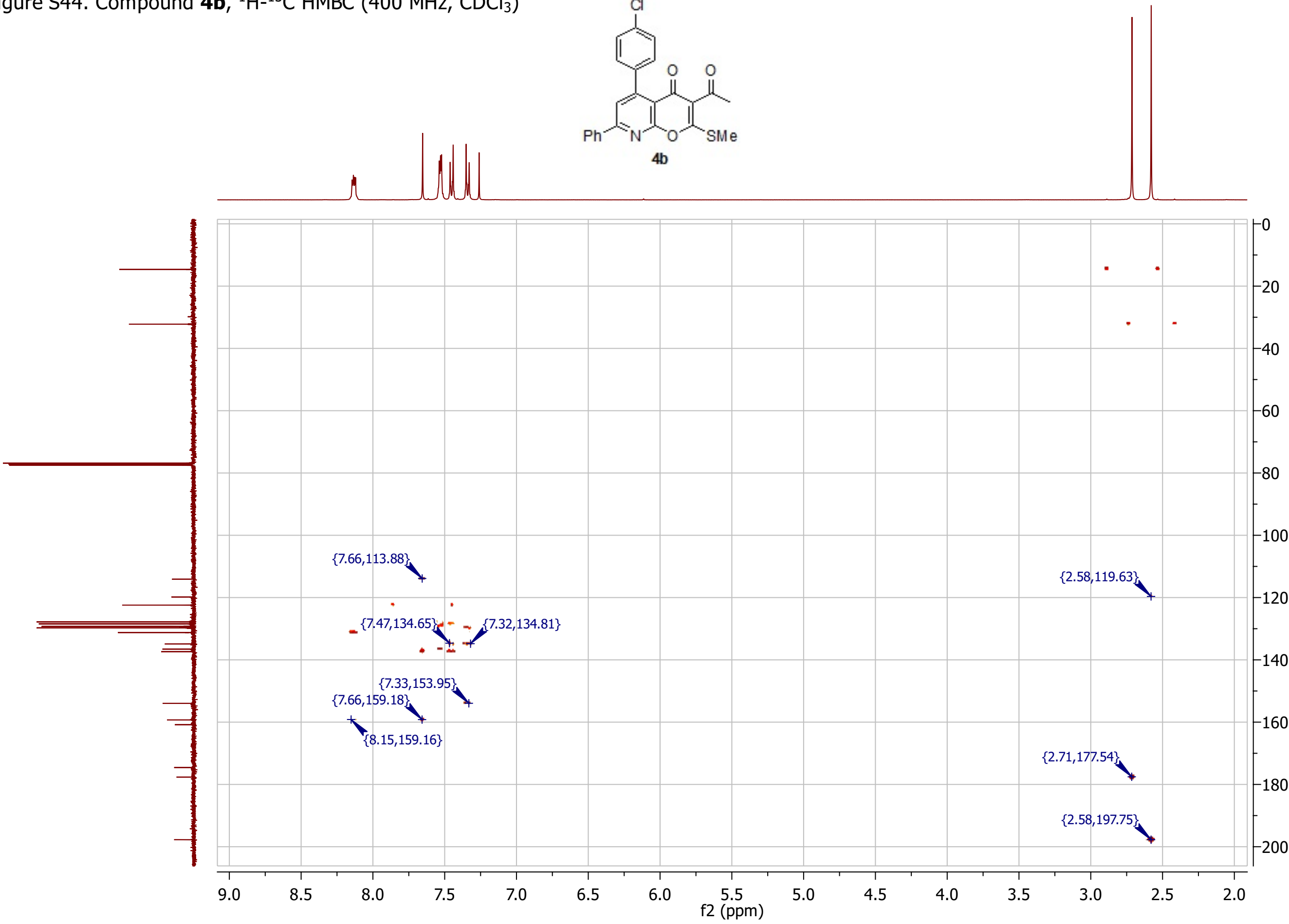

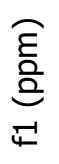




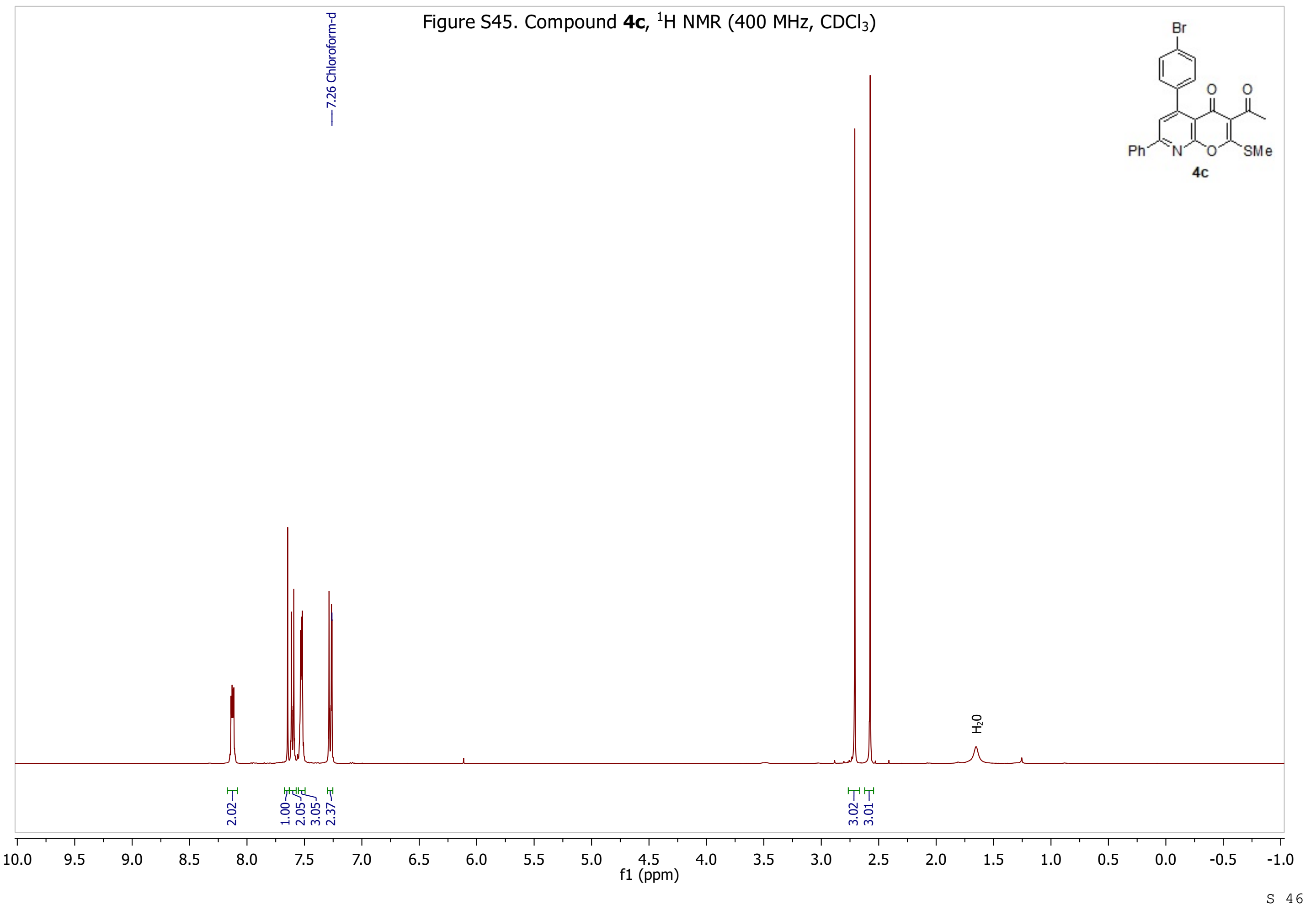


Figure S46. Compound $\mathbf{4 c},{ }^{13} \mathrm{C}\left\{{ }^{1} \mathrm{H}\right\}$ NMR $\left(101 \mathrm{MHz}, \mathrm{CDCl}_{3}\right)$

落

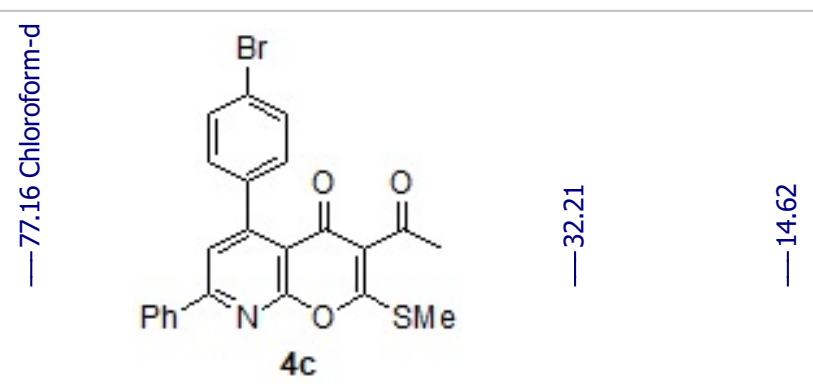

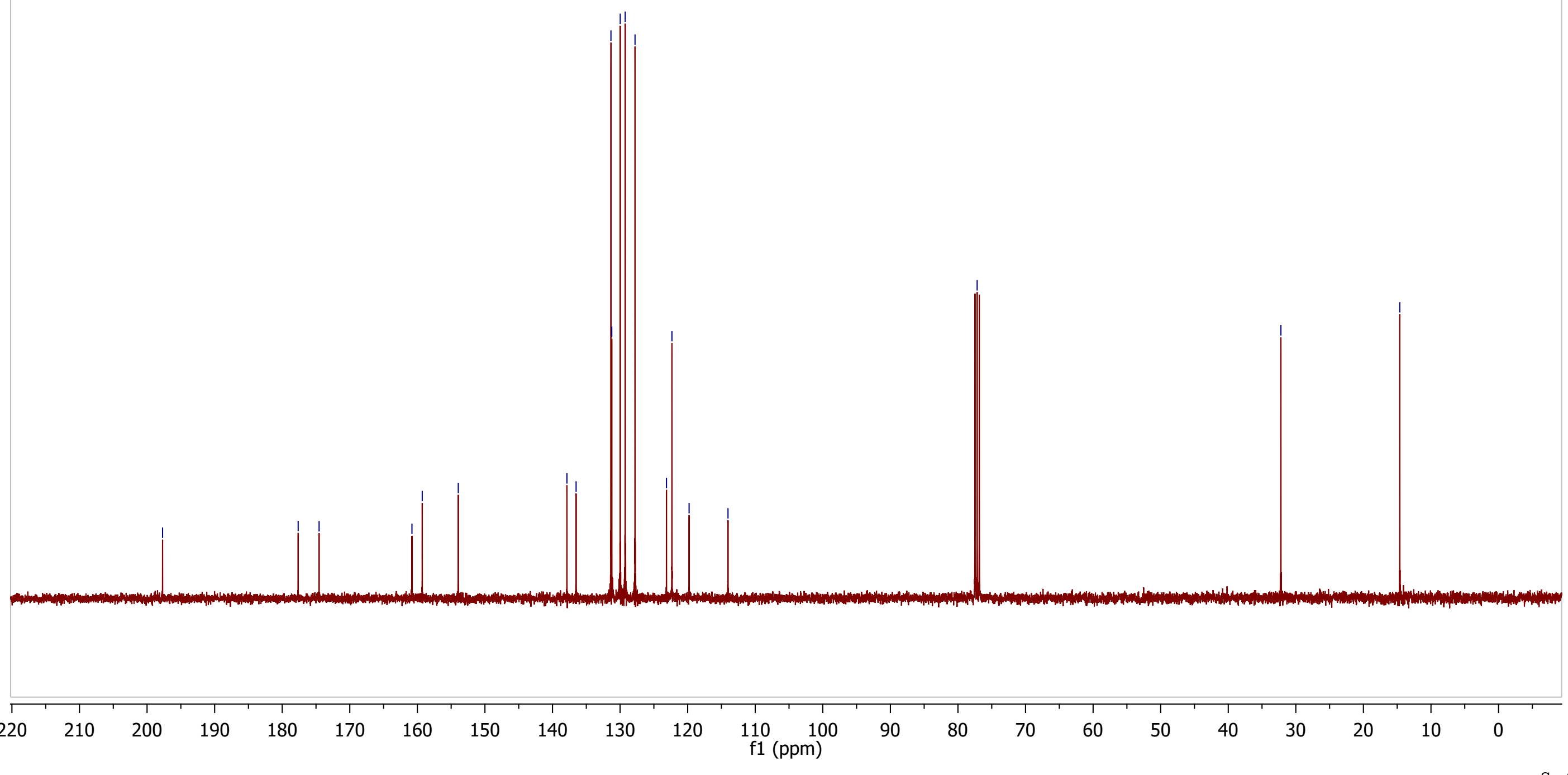

S 47 
Figure S47. Compound $4 \mathrm{c},{ }^{1} \mathrm{H}^{13} \mathrm{C} \mathrm{HMBC}\left(400 \mathrm{MHz}, \mathrm{CDCl}_{3}\right)$

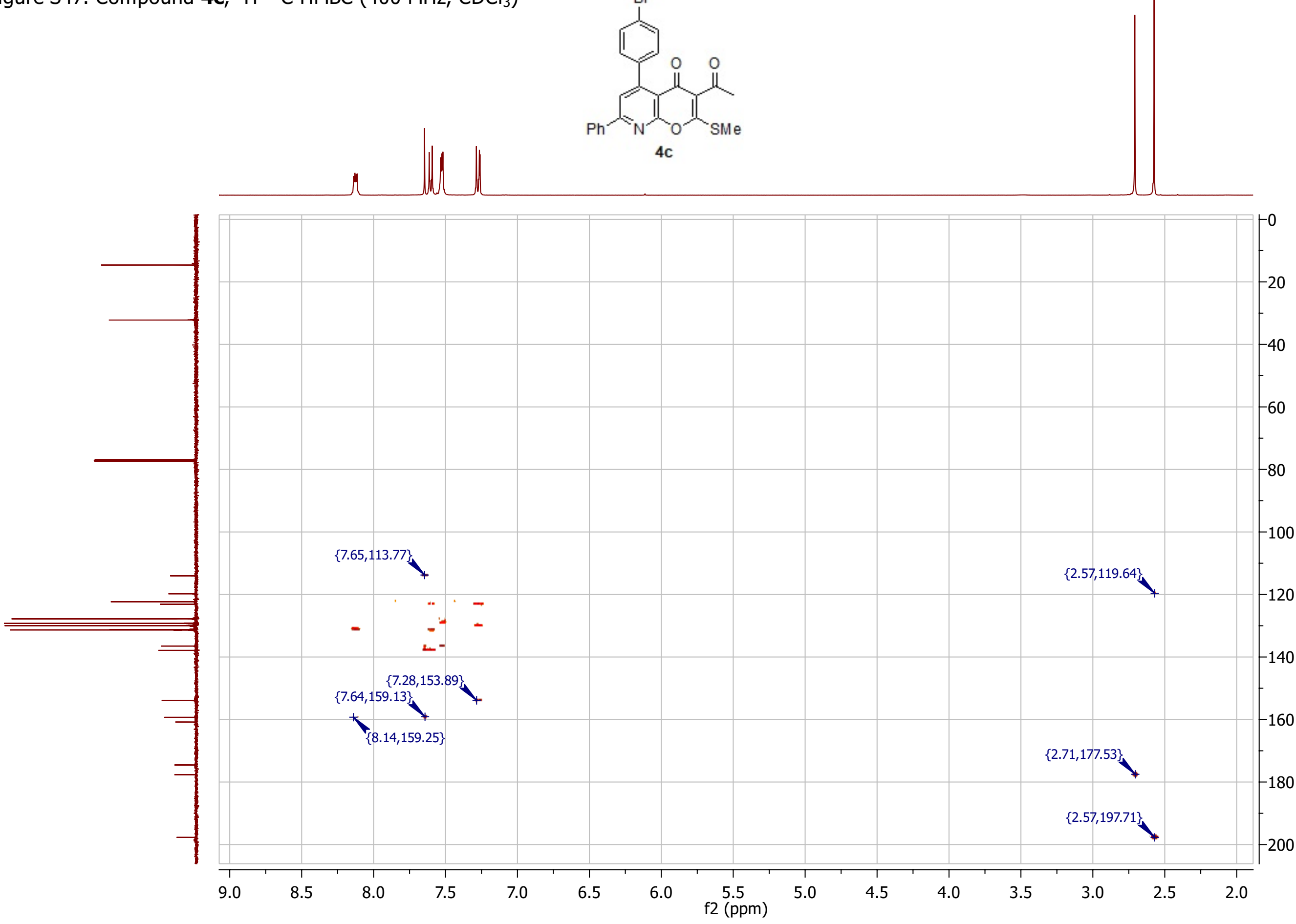

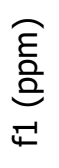



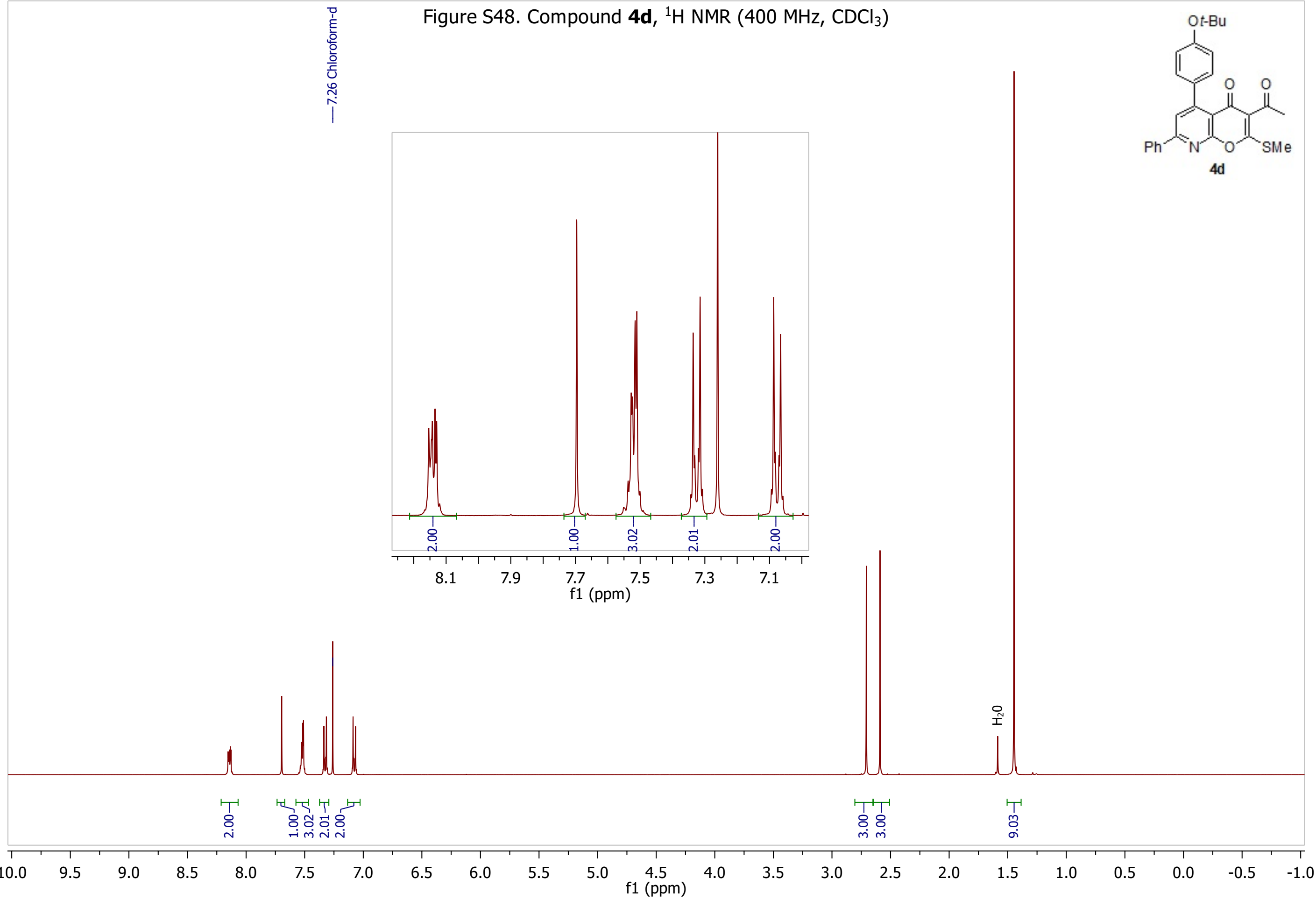
Figure S49. Compound 4d, ${ }^{13} \mathrm{C}\left\{{ }^{1} \mathrm{H}\right\}$ NMR $\left(101 \mathrm{MHz}, \mathrm{CDCl}_{3}\right)$

\begin{tabular}{|c|c|c|c|}
\hline & 孚 & 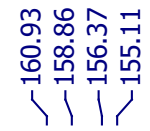 & 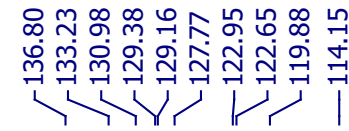 \\
\hline
\end{tabular}

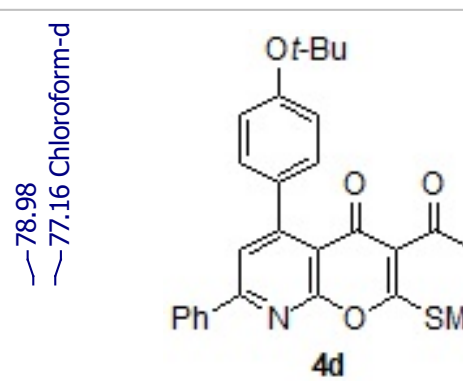

ㄱำ

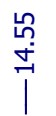

$4 d$

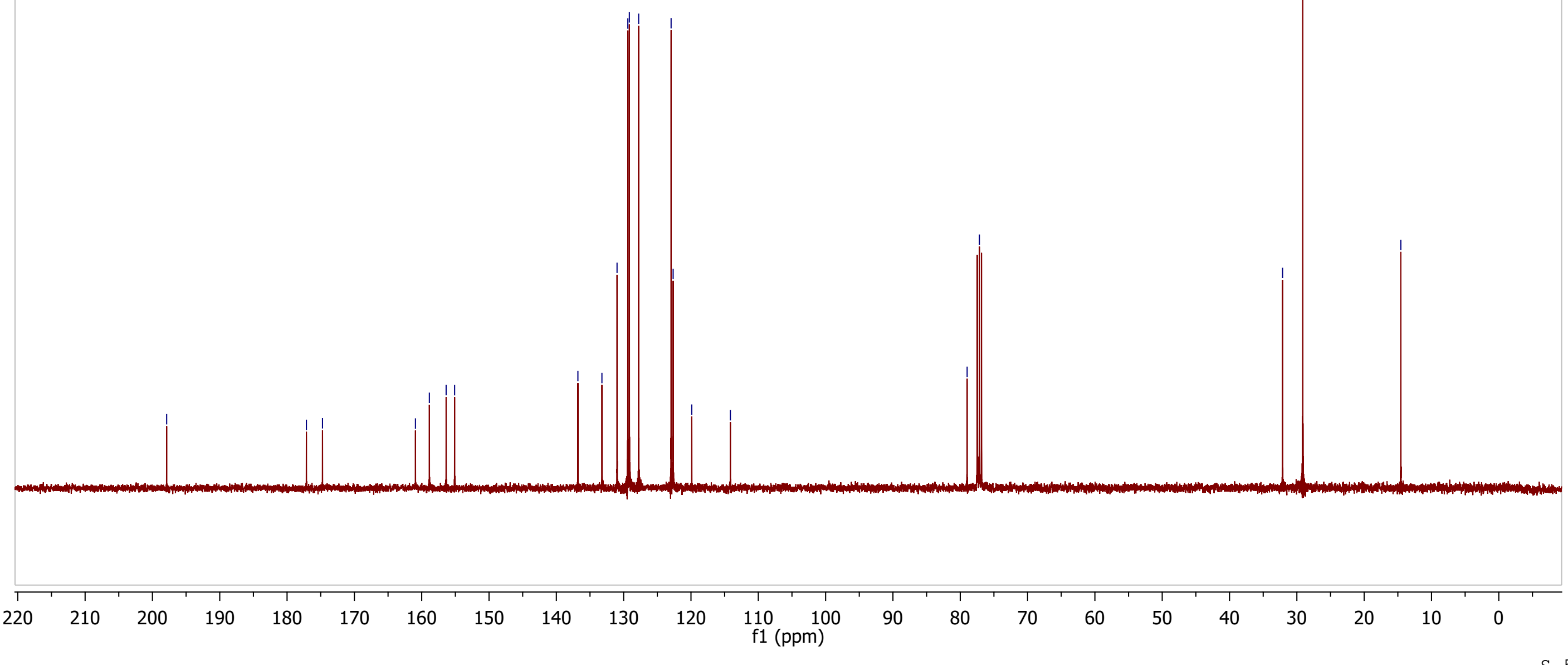


Figure S50. Compound 4d, ${ }^{1} \mathrm{H}-{ }^{13} \mathrm{C}$ HMBC $\left(400 \mathrm{MHz}, \mathrm{CDCl}_{3}\right)$
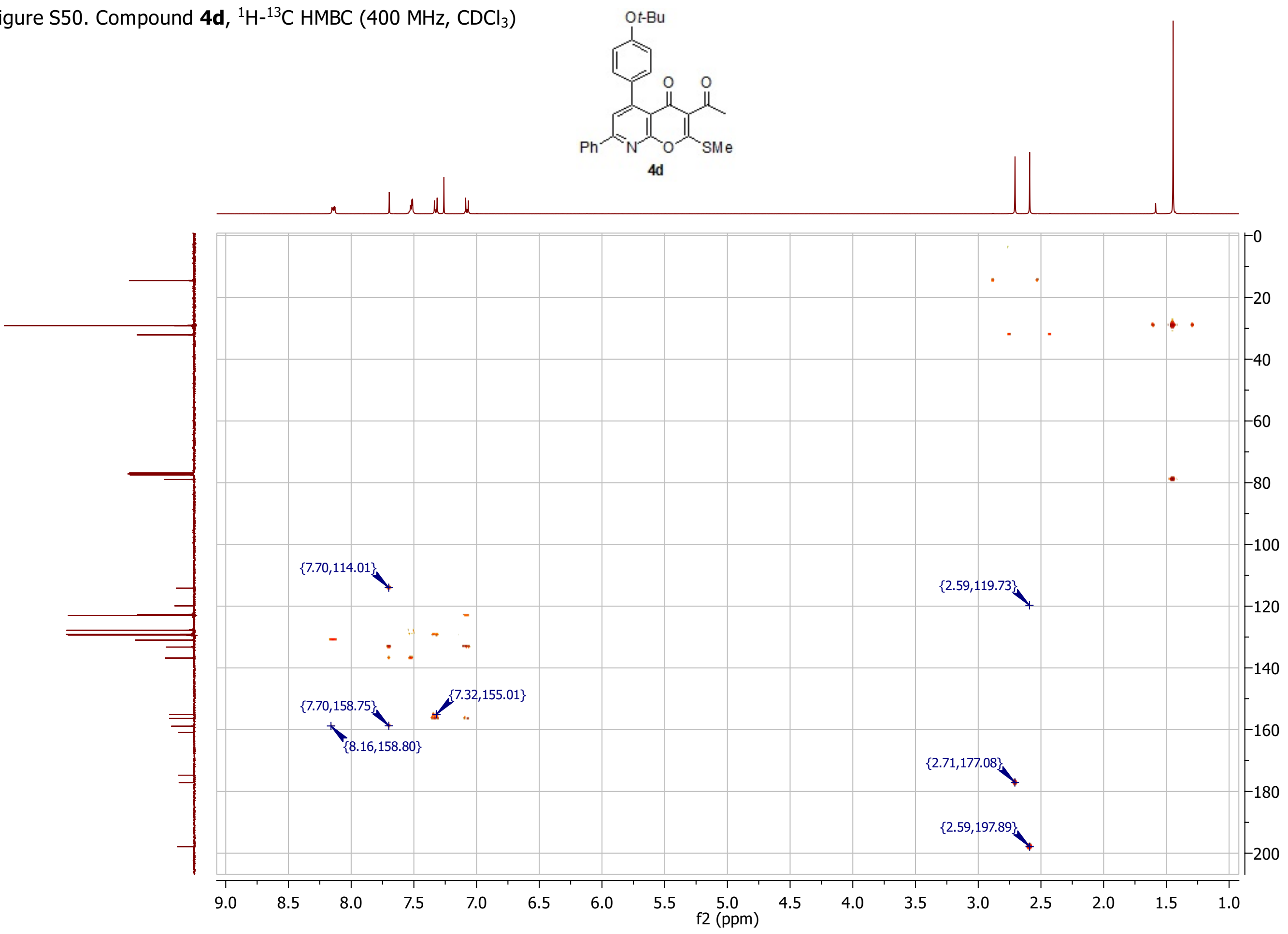

$\hat{E}$
믄
$\overline{4}$ 


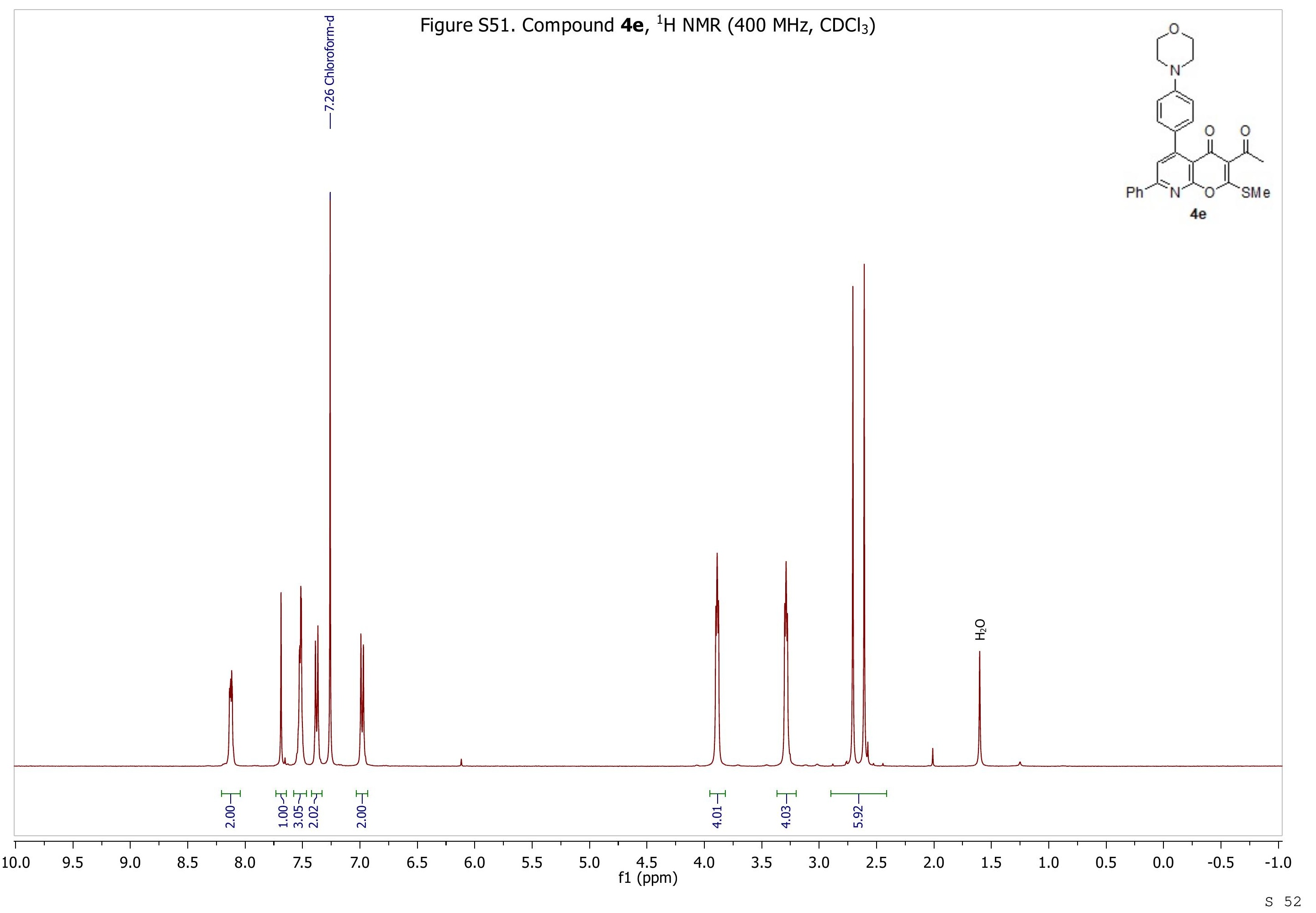


Figure S52. Compound $4 \mathbf{e},{ }^{13} \mathrm{C}\left\{{ }^{1} \mathrm{H}\right\}$ NMR $\left(101 \mathrm{MHz}, \mathrm{CDCl}_{3}\right)$
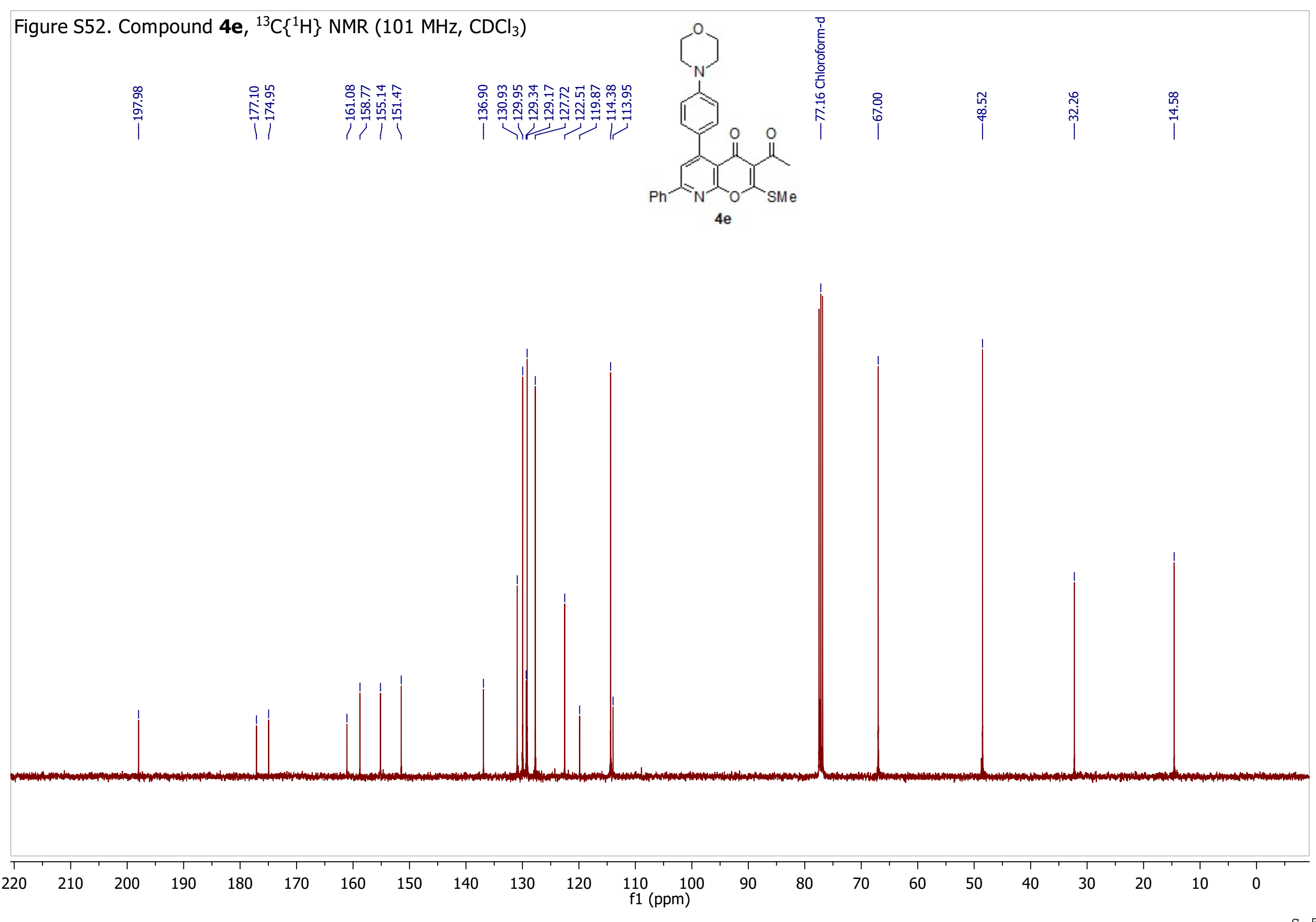


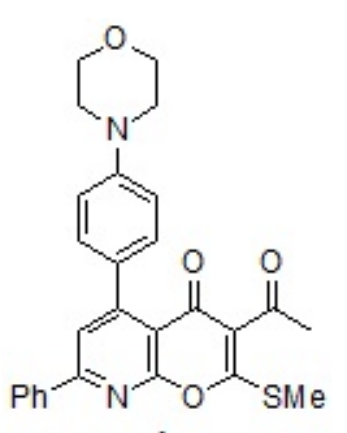

$4 \mathrm{e}$

Figure S53. Compound $4 \mathbf{4},{ }^{1} \mathrm{H}-{ }^{13} \mathrm{C} \mathrm{HSQC}\left(400 \mathrm{MHz}, \mathrm{CDCl}_{3}\right)$

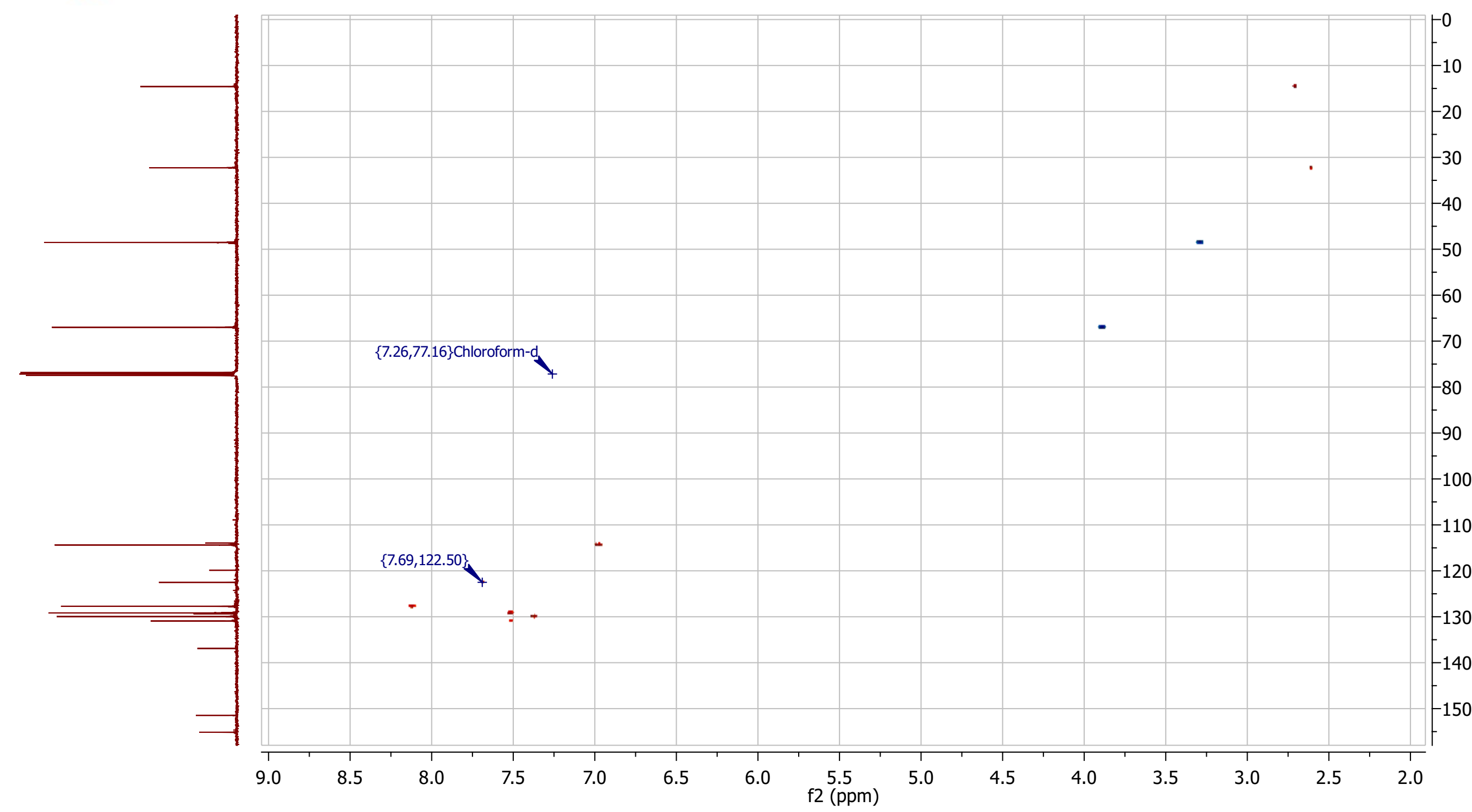




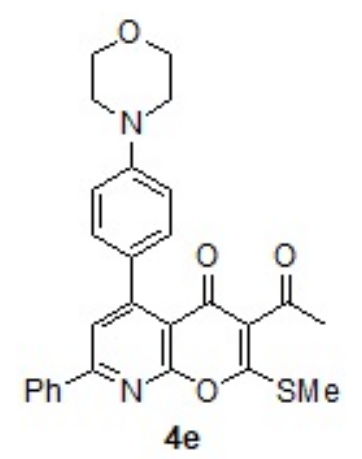

Figure S54. Compound $\mathbf{4 e},{ }^{1} \mathrm{H}^{13} \mathrm{C} \mathrm{HMBC}\left(400 \mathrm{MHz}, \mathrm{CDCl}_{3}\right)$

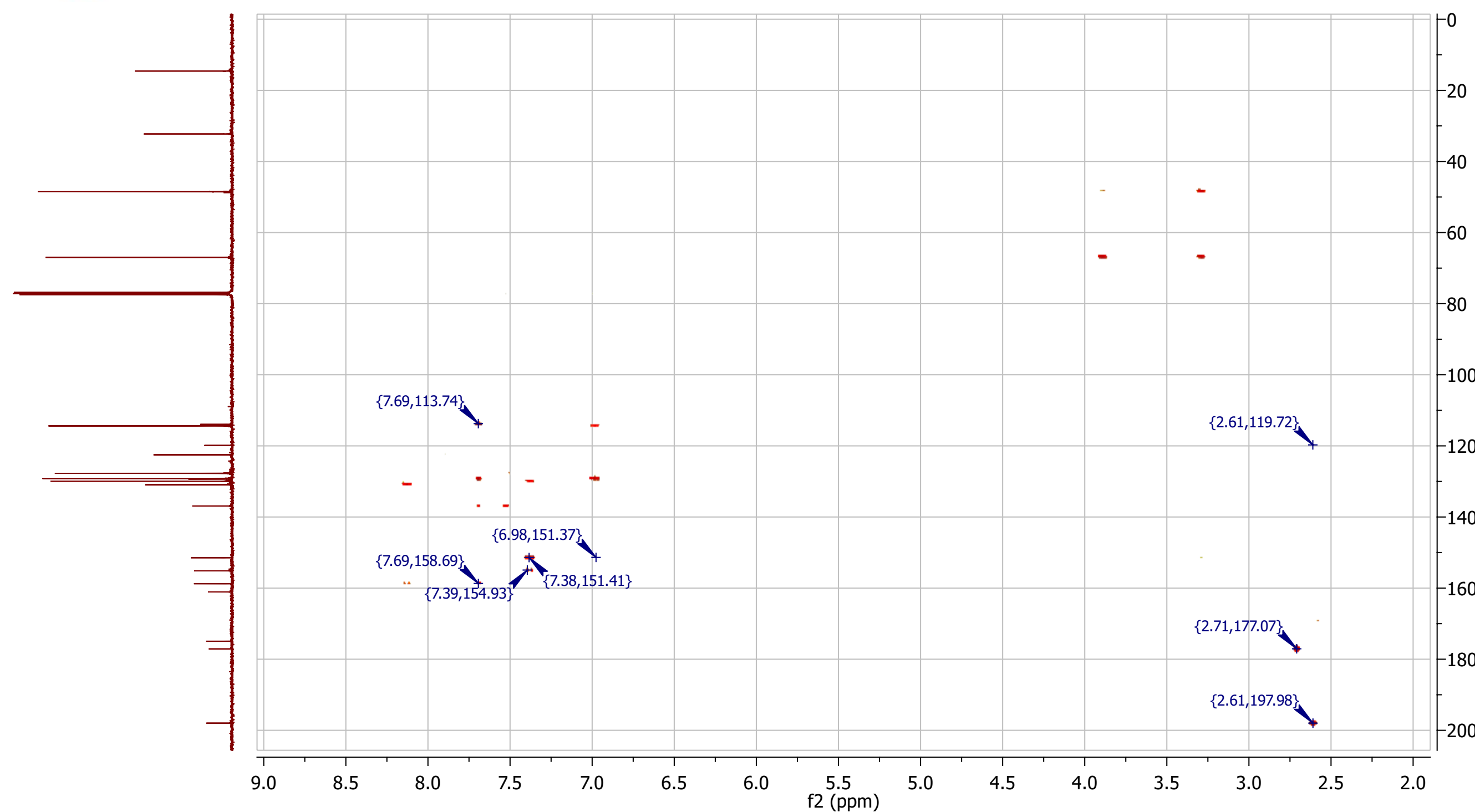

$\hat{\mathrm{E}}$
흔 


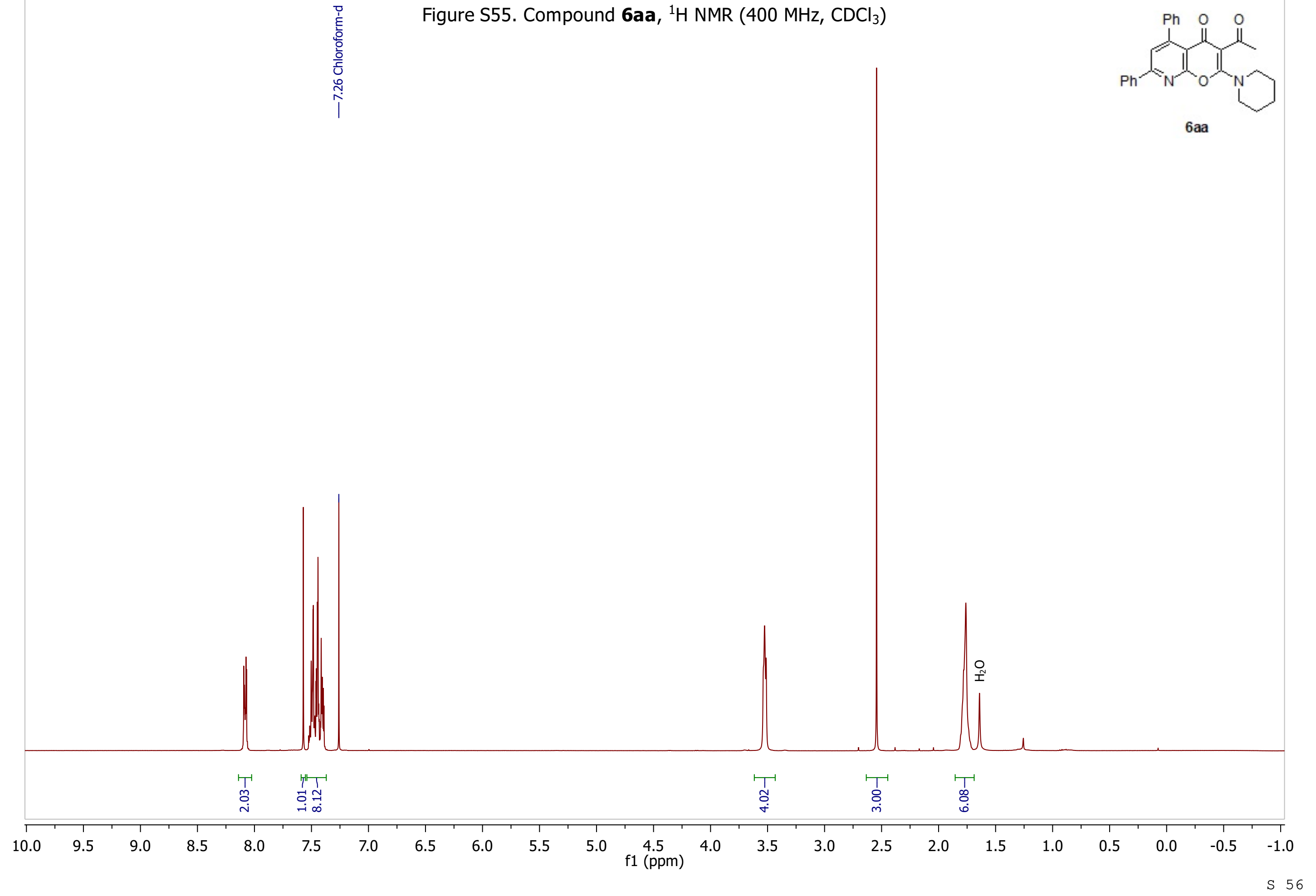




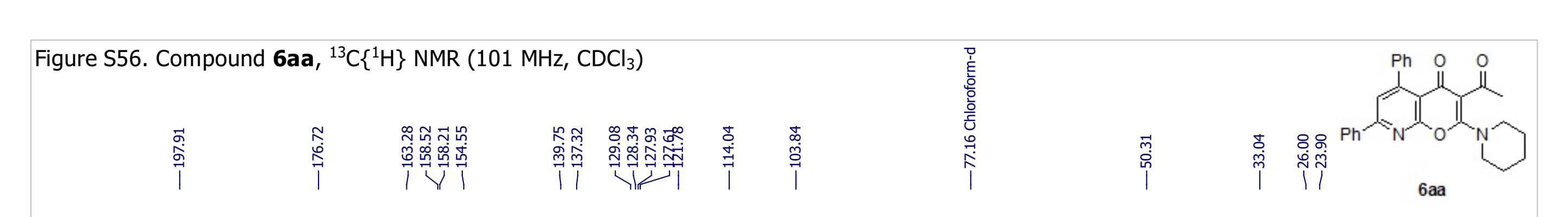

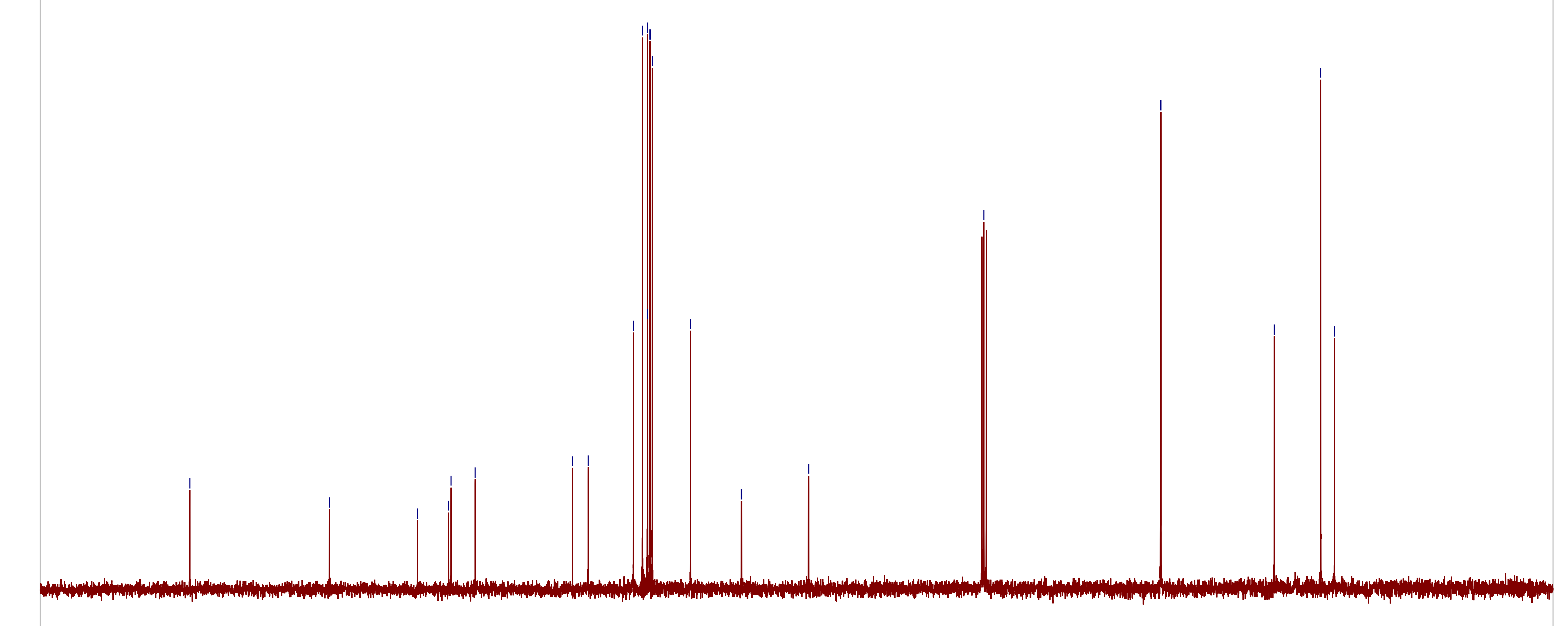




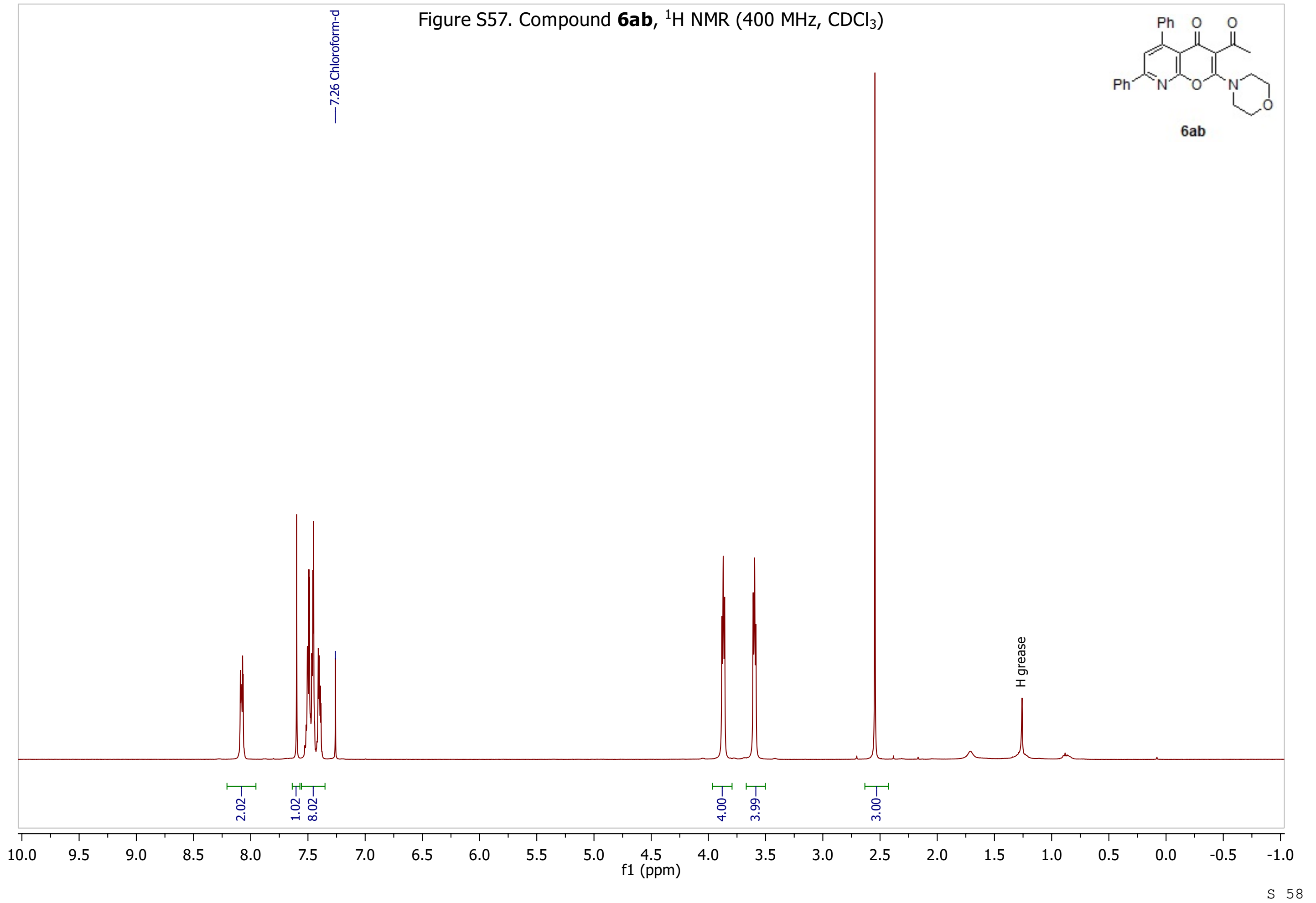


Figure S58. Compound $\mathbf{6 a b},{ }^{13} \mathrm{C}\left\{{ }^{1} \mathrm{H}\right\}$ NMR $\left(101 \mathrm{MHz}, \mathrm{CDCl}_{3}\right)$

\begin{tabular}{|c|c|c|c|c|}
\hline & $\begin{array}{l}8 \\
0 \\
0 \\
0 \\
1\end{array}$ & 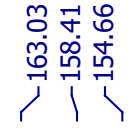 & 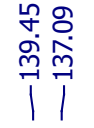 & 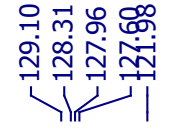 \\
\hline
\end{tabular}

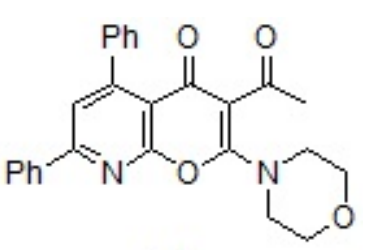

$6 a b$

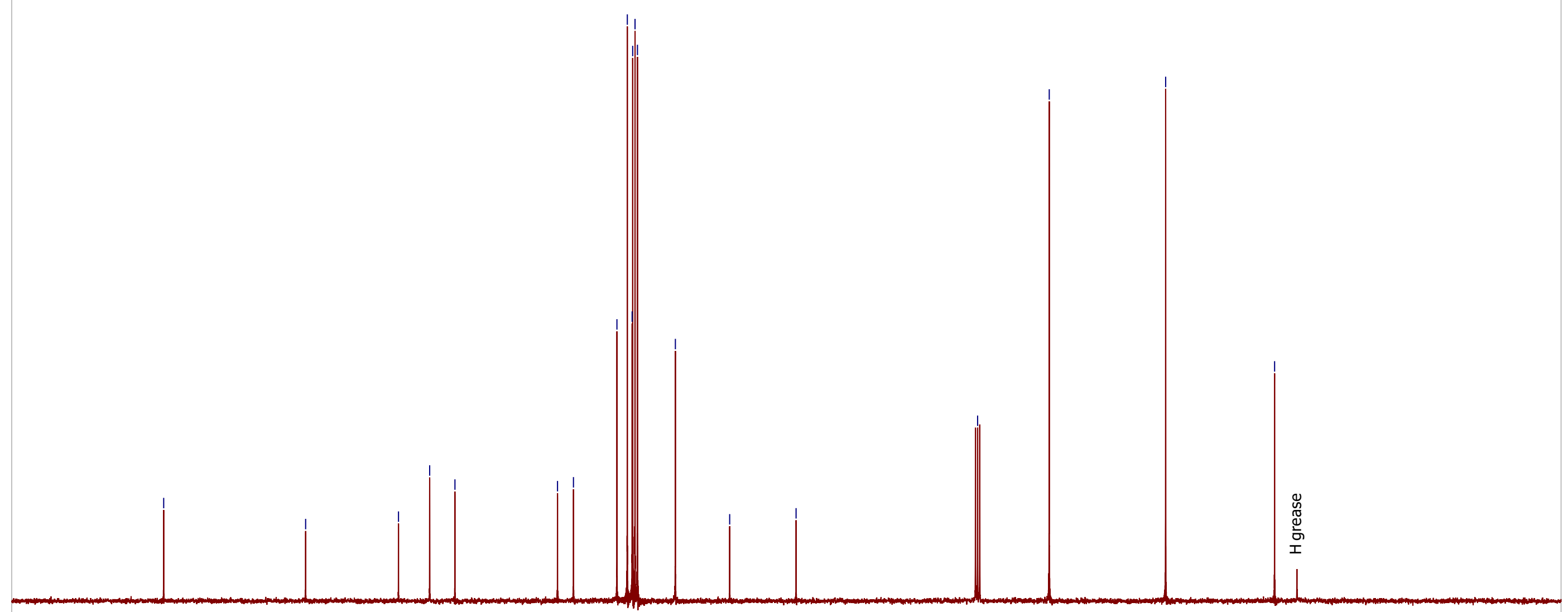



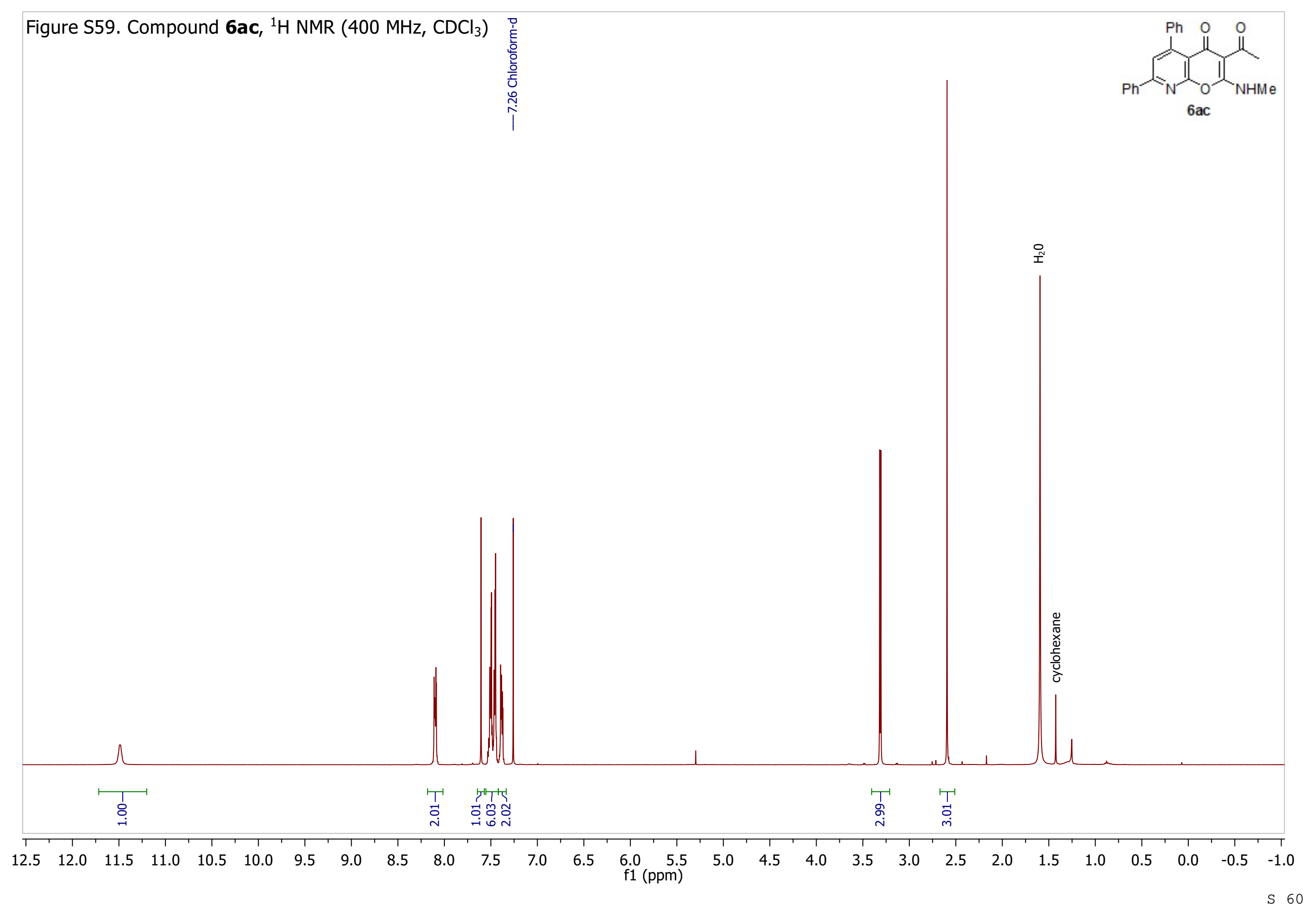
Figure S60. Compound 6ac, ${ }^{13} \mathrm{C}\left\{{ }^{1} \mathrm{H}\right\}$ NMR $\left(101 \mathrm{MHz}, \mathrm{CDCl}_{3}\right)$

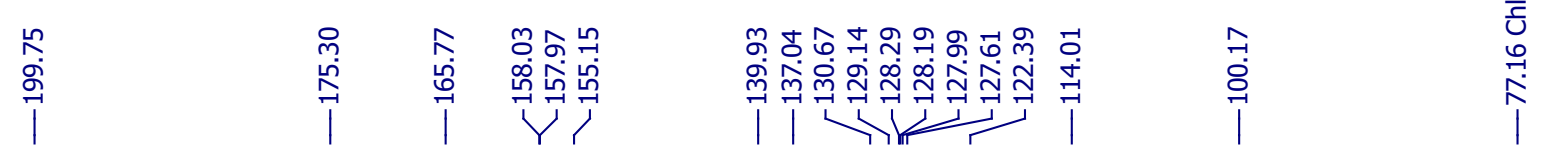

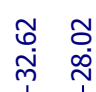
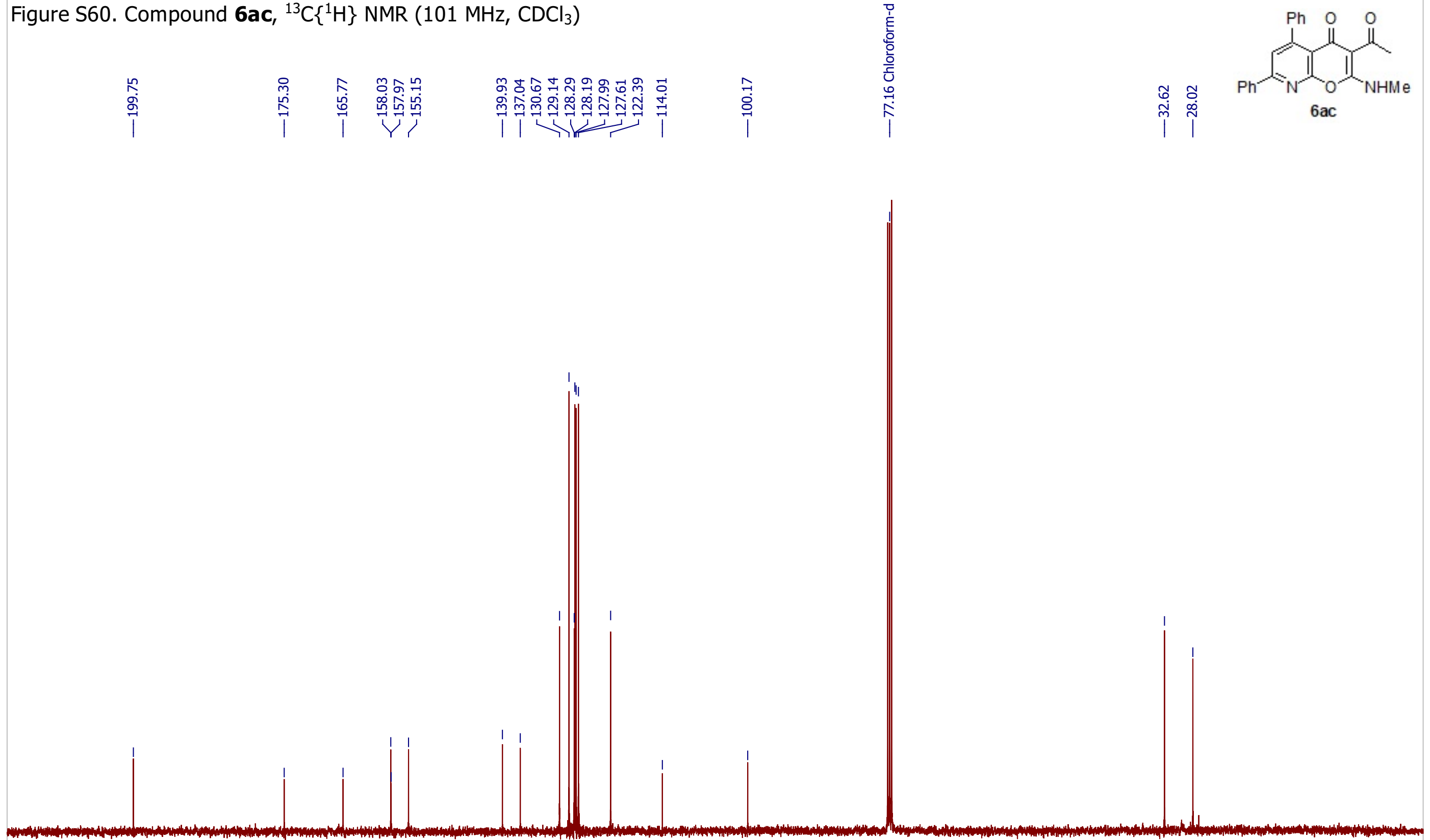

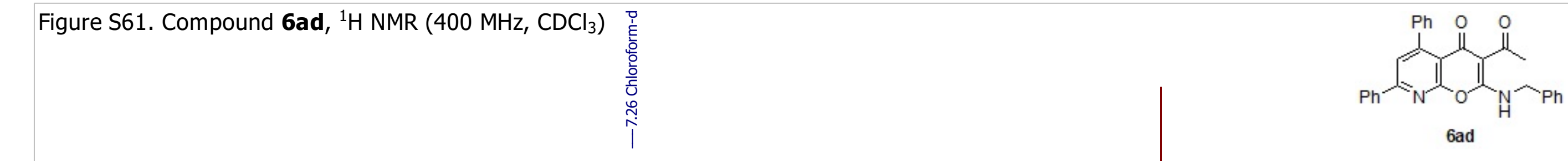

6ad

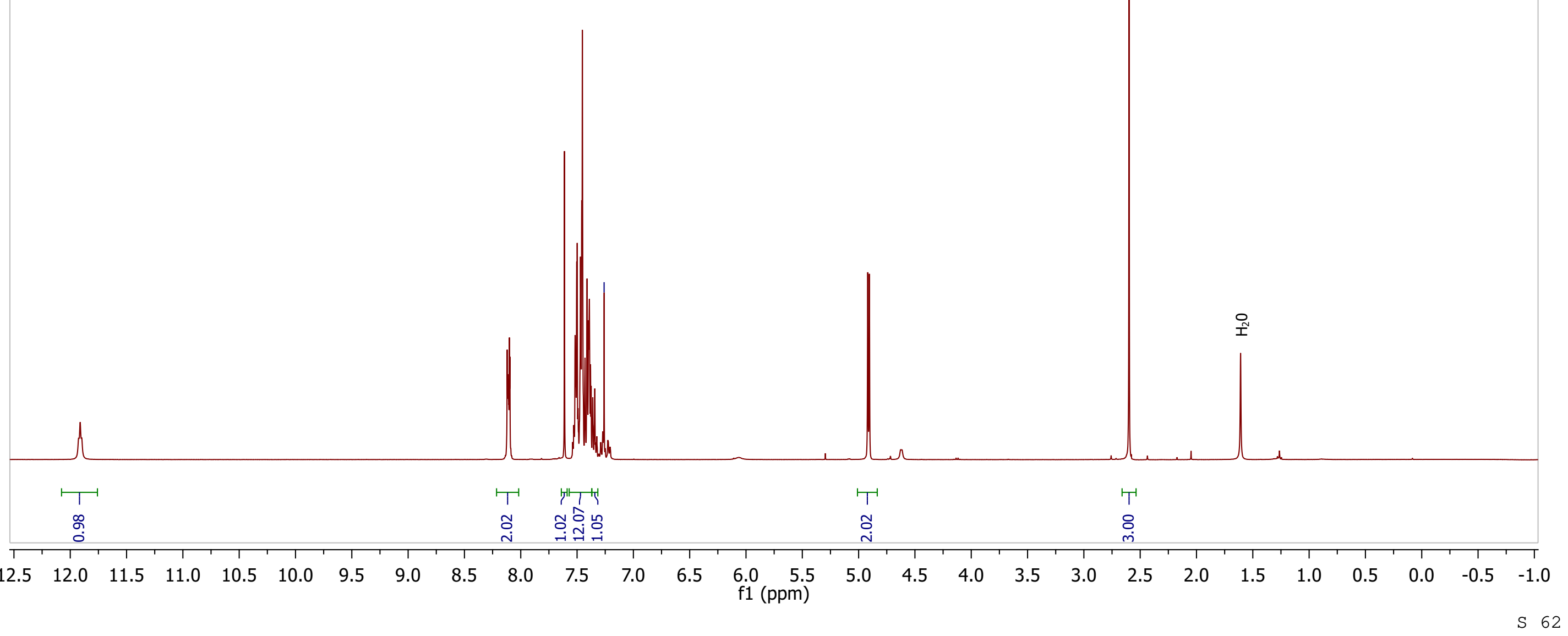


Figure S62. Compound 6ad, ${ }^{13} \mathrm{C}\left\{{ }^{1} \mathrm{H}\right\}$ NMR $\left(101 \mathrm{MHz}, \mathrm{CDCl}_{3}\right)$

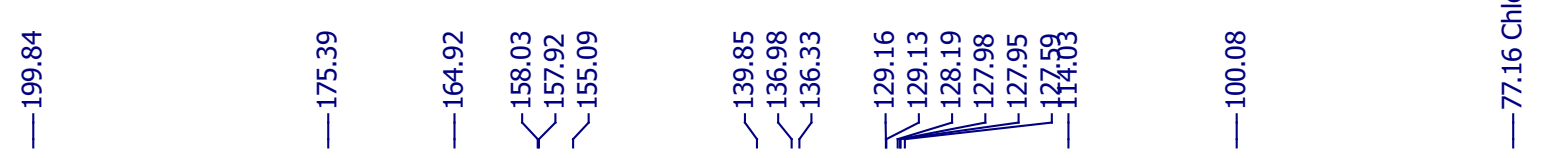

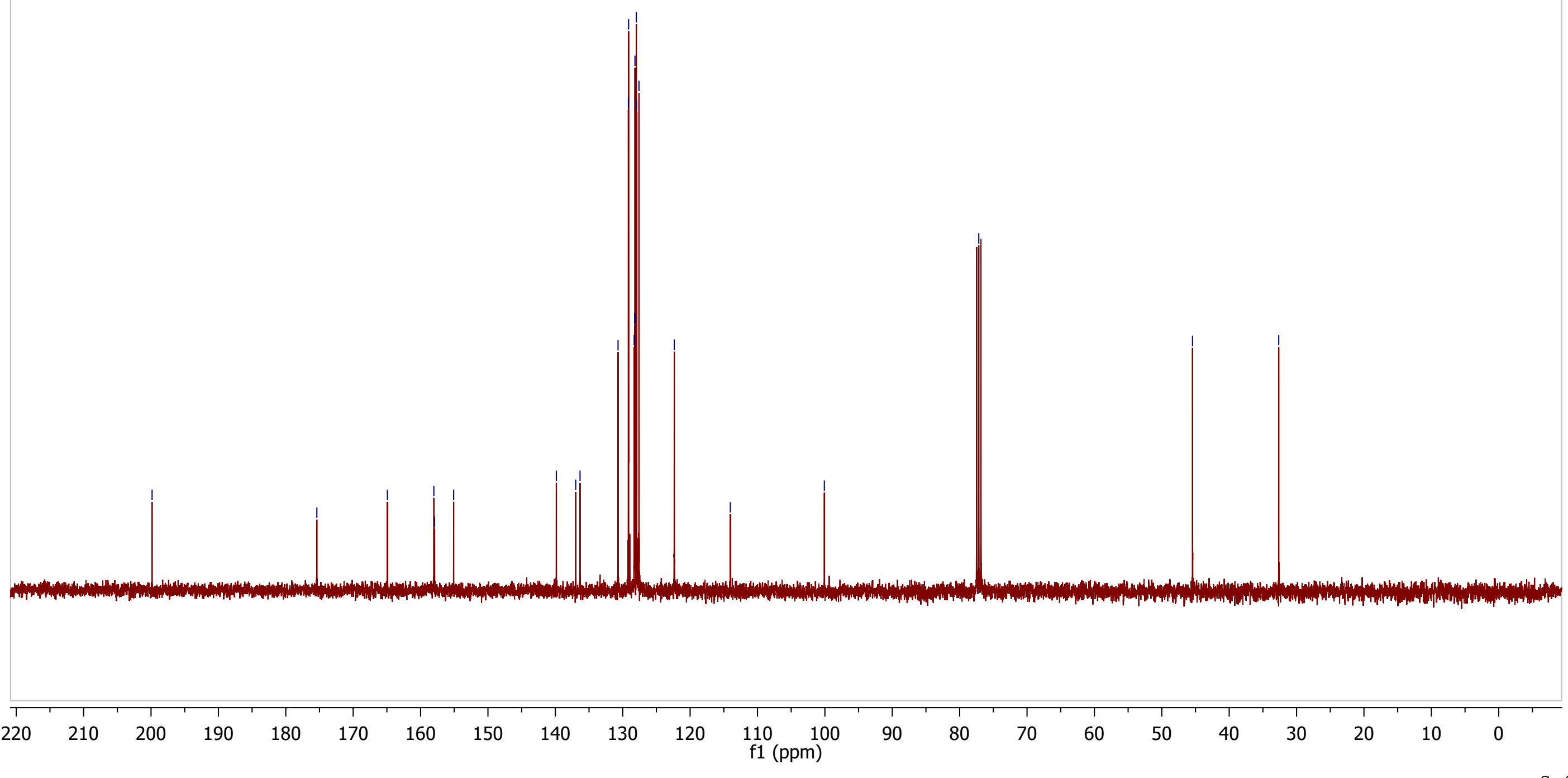


Figure S63. Compound 6ad, ${ }^{1} \mathrm{H}^{13} \mathrm{C} \mathrm{HMBC}\left(400 \mathrm{MHz}, \mathrm{CDCl}_{3}\right)$
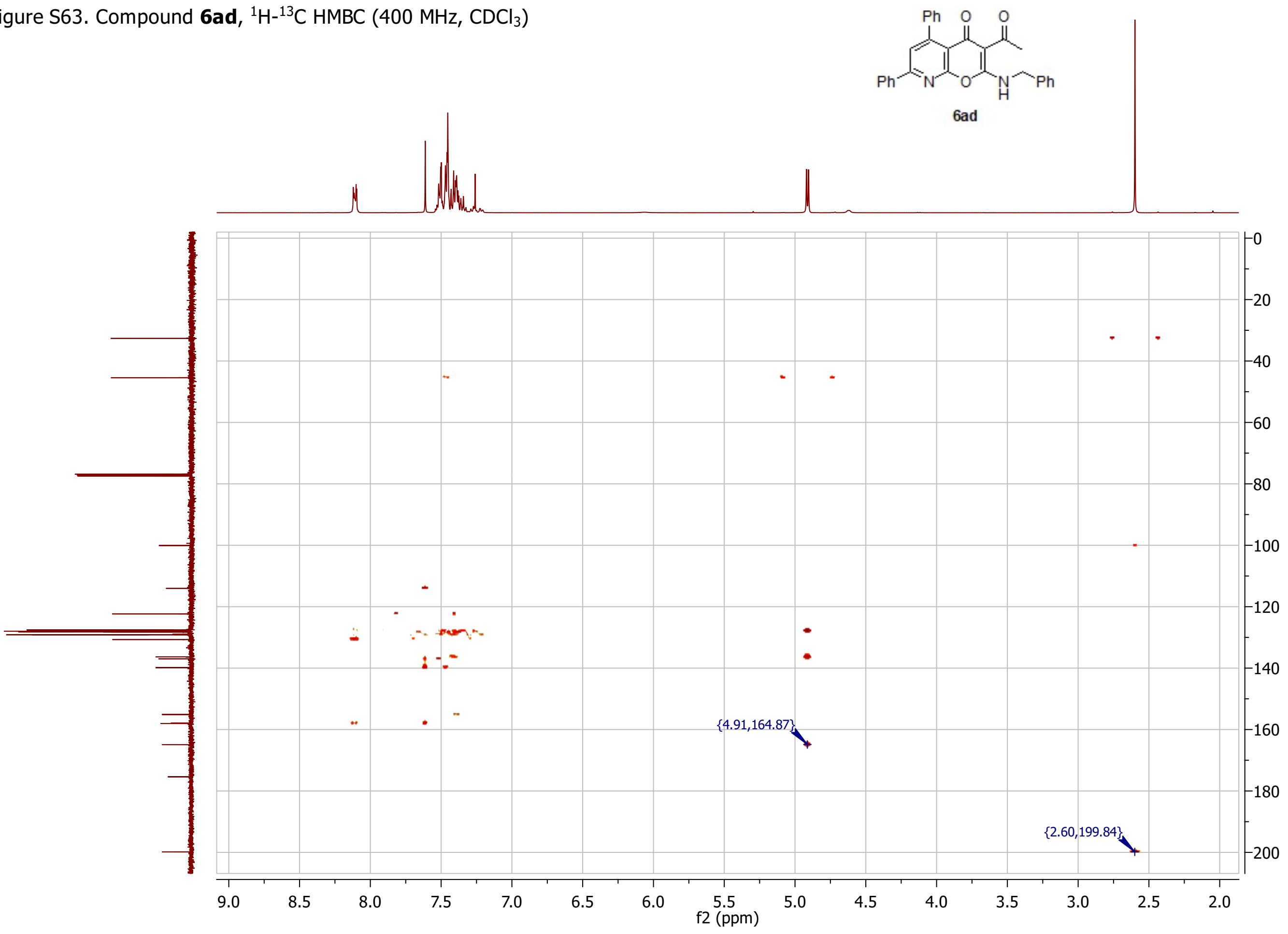

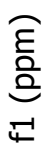


Figure S64. Compound 6ae, ${ }^{1} \mathrm{H}$ NMR (400 MHz, DMSO-d 6 )

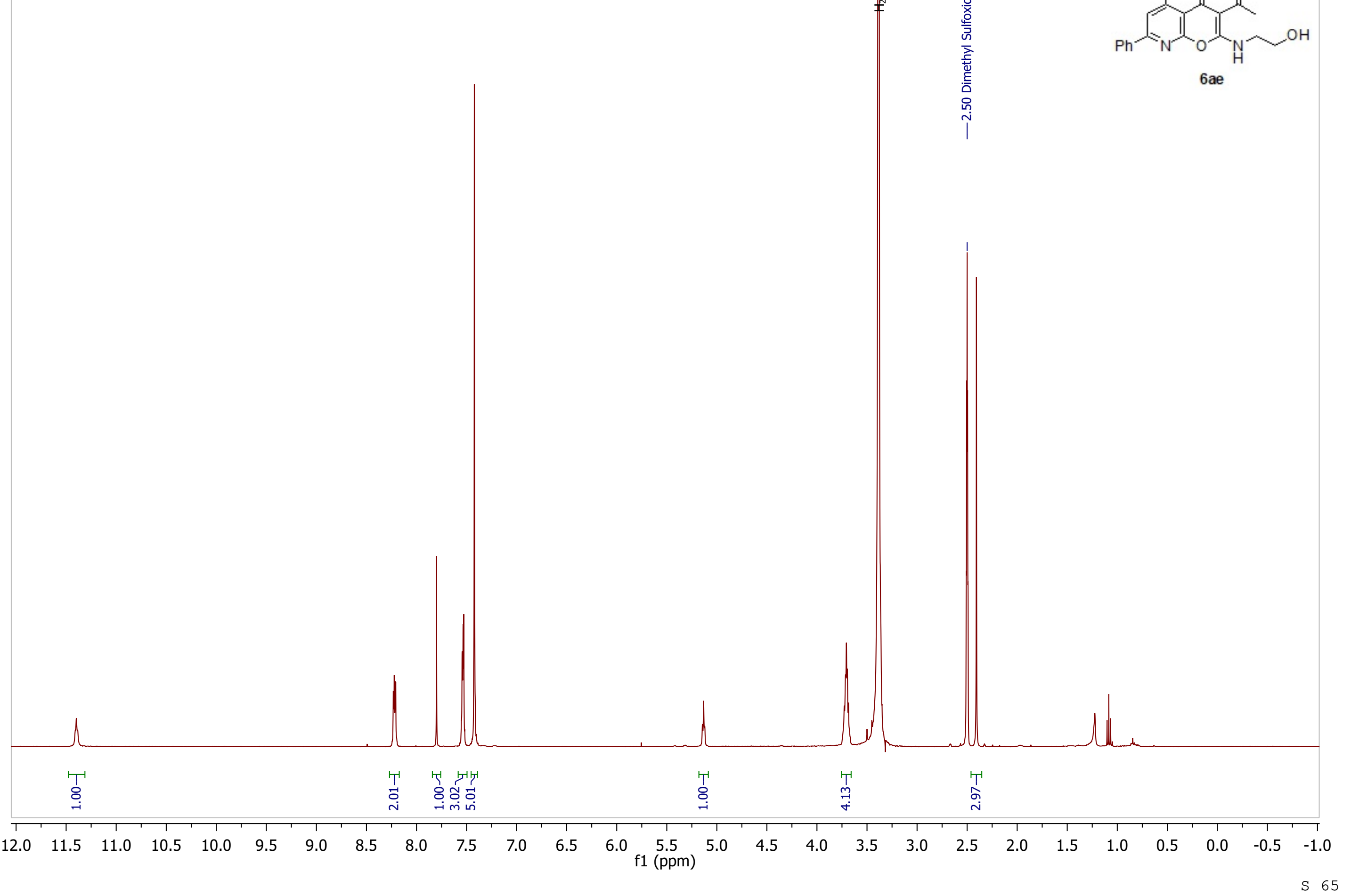



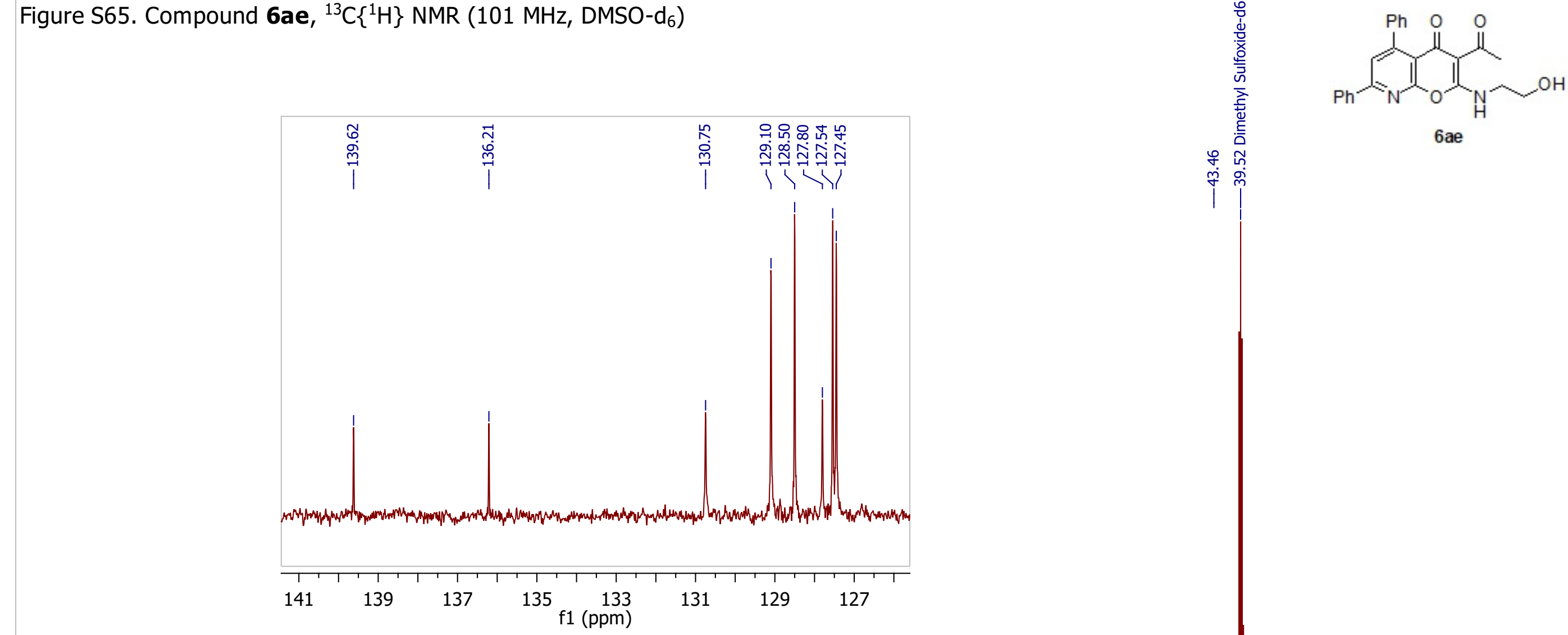

$6 a e$

พั กิ

พं

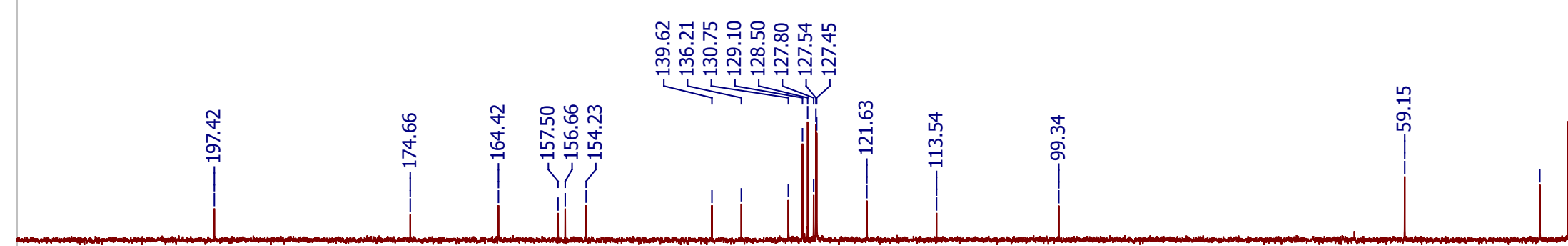



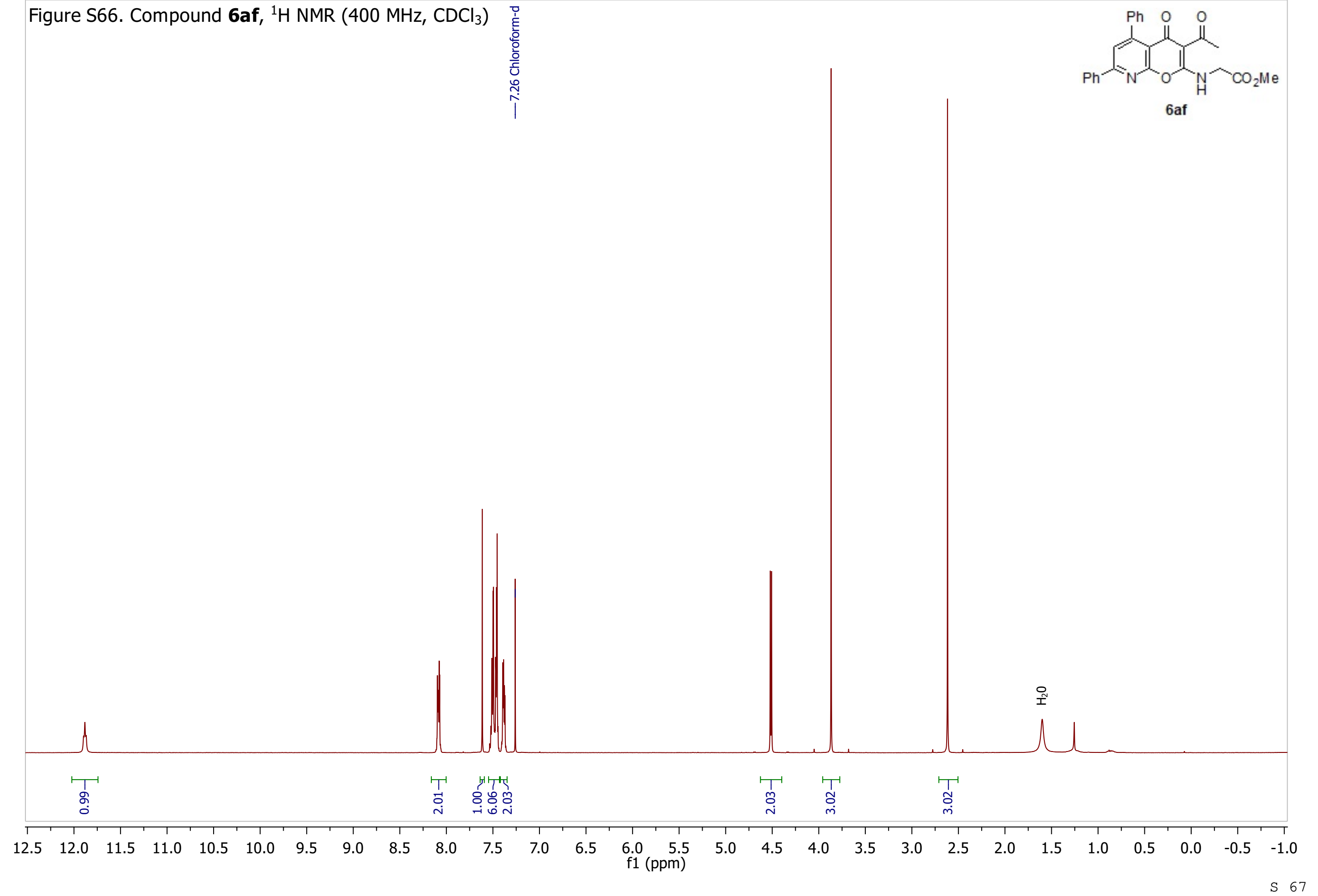
Figure S67. Compound 6af, ${ }^{13} \mathrm{C}\left\{{ }^{1} \mathrm{H}\right\} \mathrm{NMR}\left(101 \mathrm{MHz}, \mathrm{CDCl}_{3}\right)$

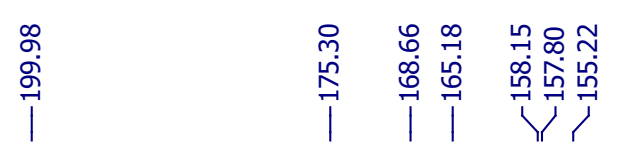

等

$\stackrel{\text { g }}{\stackrel{0}{0}}$

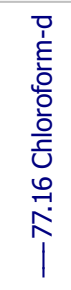
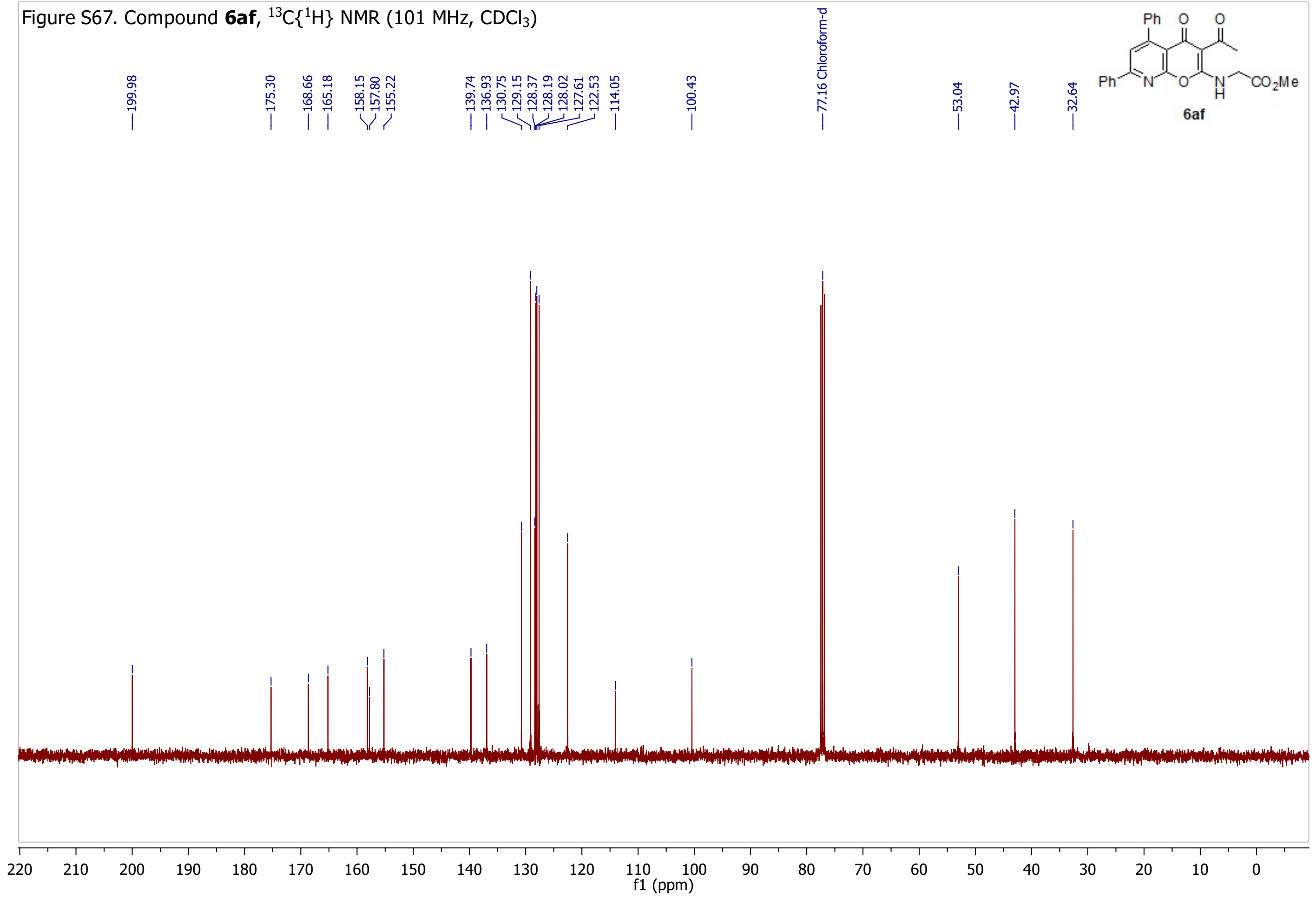

S 68 

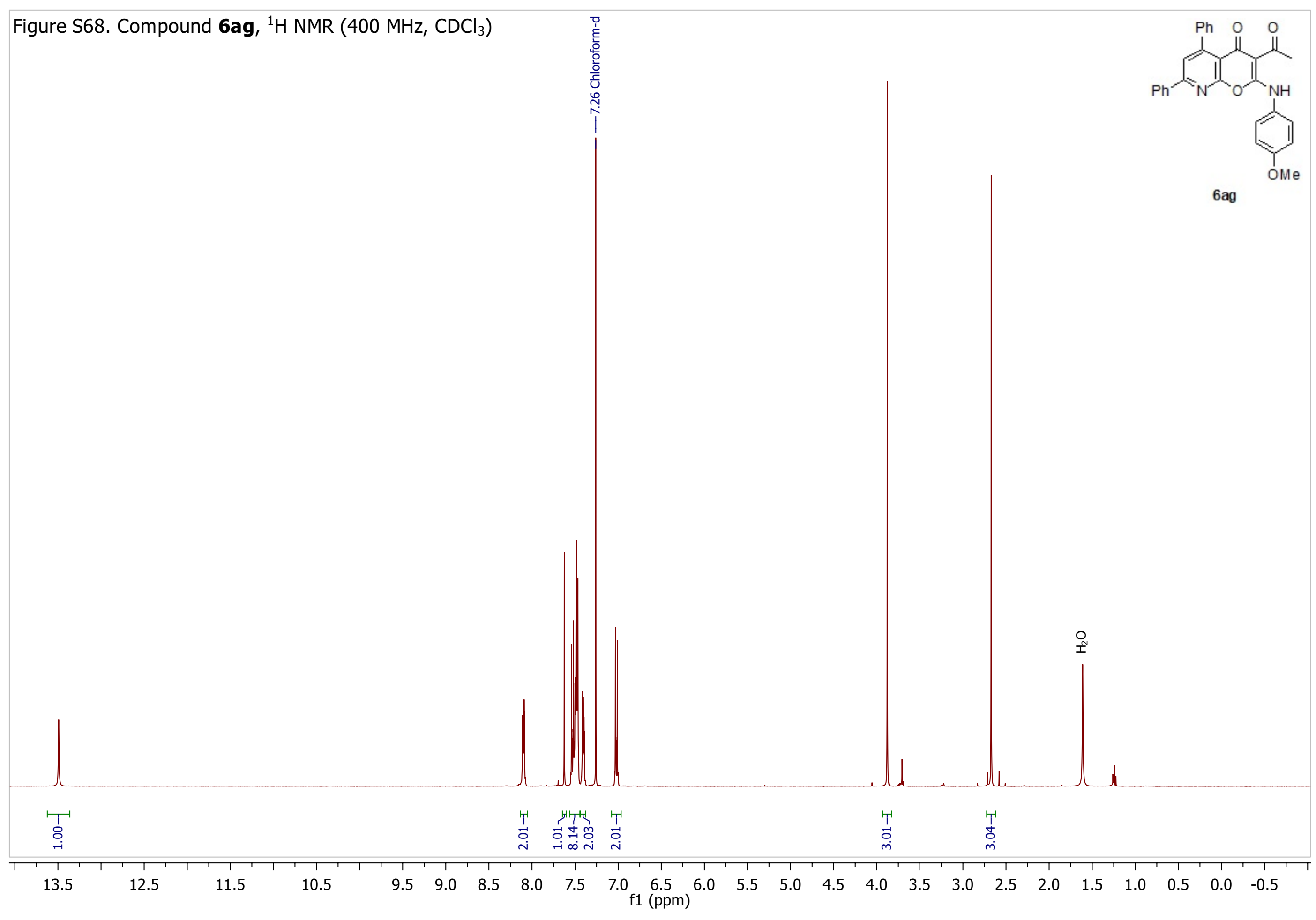
Figure S69. Compound $\mathbf{6 a g},{ }^{13} \mathrm{C}\left\{{ }^{1} \mathrm{H}\right\}$ NMR $\left(101 \mathrm{MHz}, \mathrm{CDCl}_{3}\right)$

|

ำ

$\stackrel{\text { กิ }}{n}$

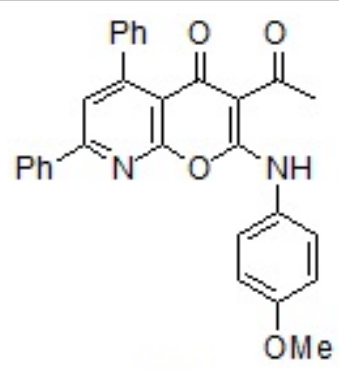

6 ag

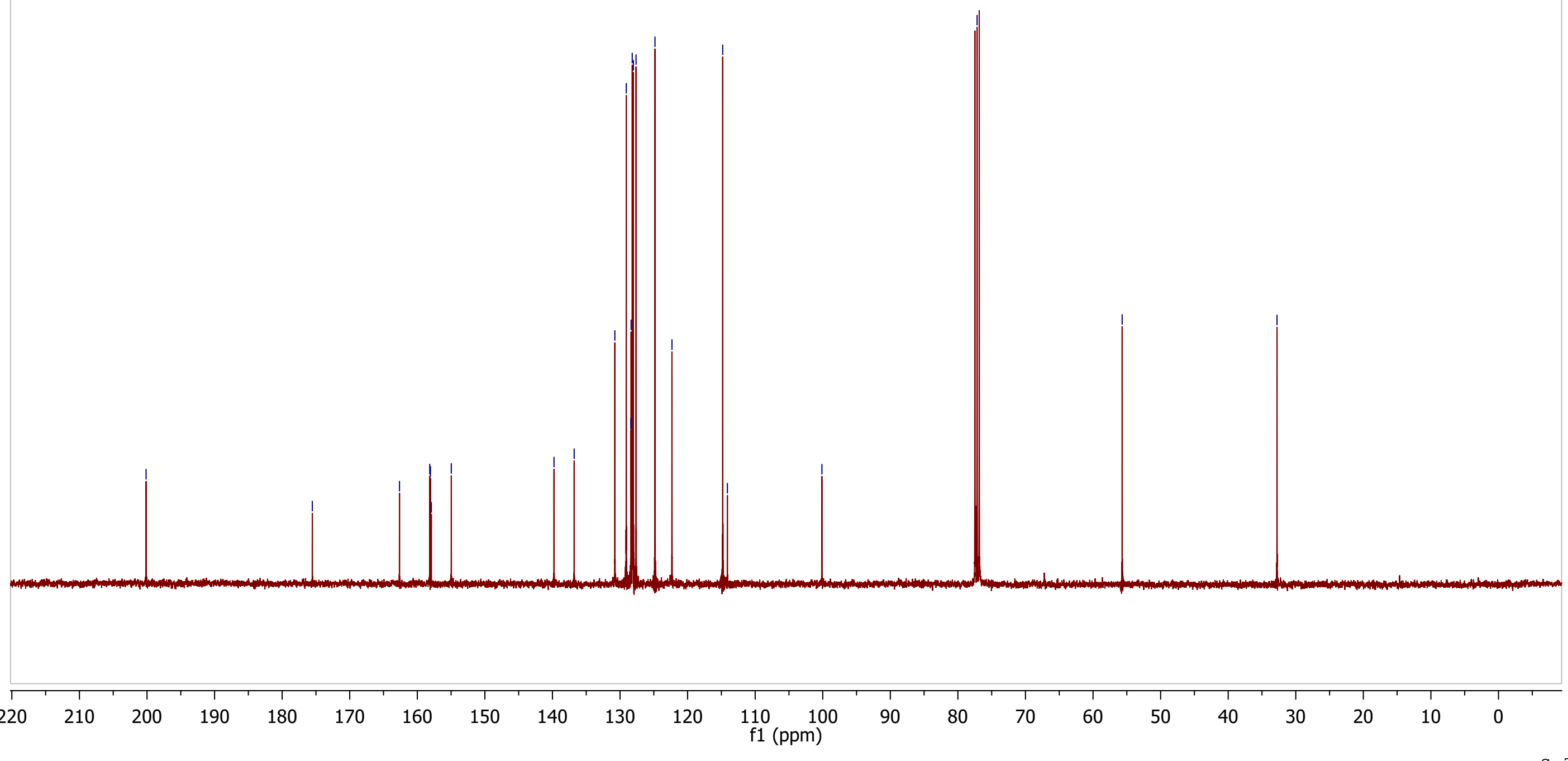

S 70 
Figure S70. Compound 6ah, ${ }^{1} \mathrm{H}$ NMR (400 MHz, $\mathrm{CDCl}_{3}$ )
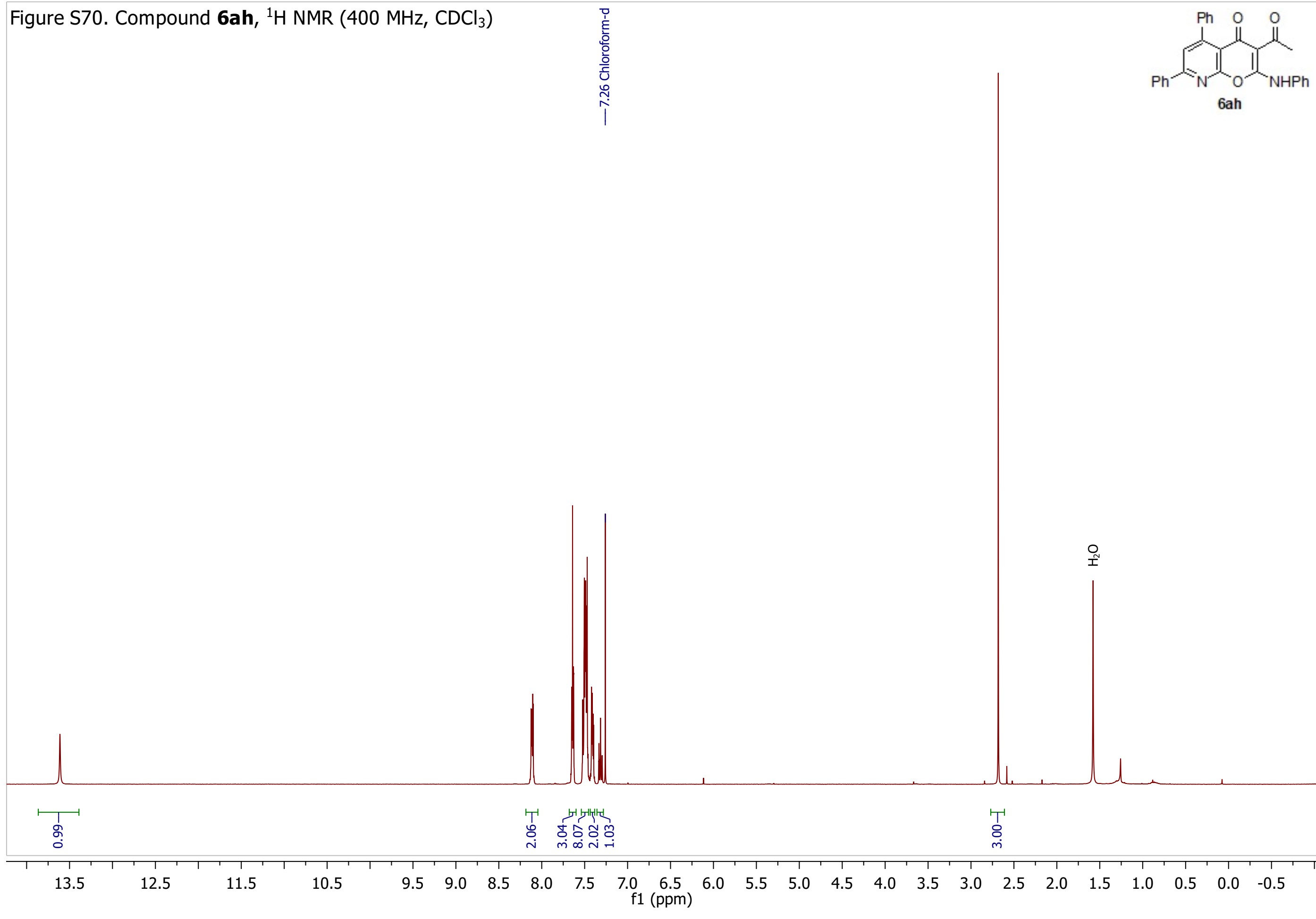
Figure S71. Compound 6ah, ${ }^{13} \mathrm{C}\left\{{ }^{1} \mathrm{H}\right\}$ NMR (101 MHz, $\left.\mathrm{CDCl}_{3}\right)$

ก்̃

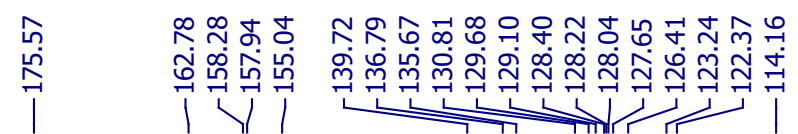

$\stackrel{\text { m. }}{\stackrel{0}{0}}$

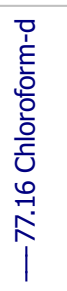

$\stackrel{\substack{n \\ \stackrel{n}{m}}}{m}$
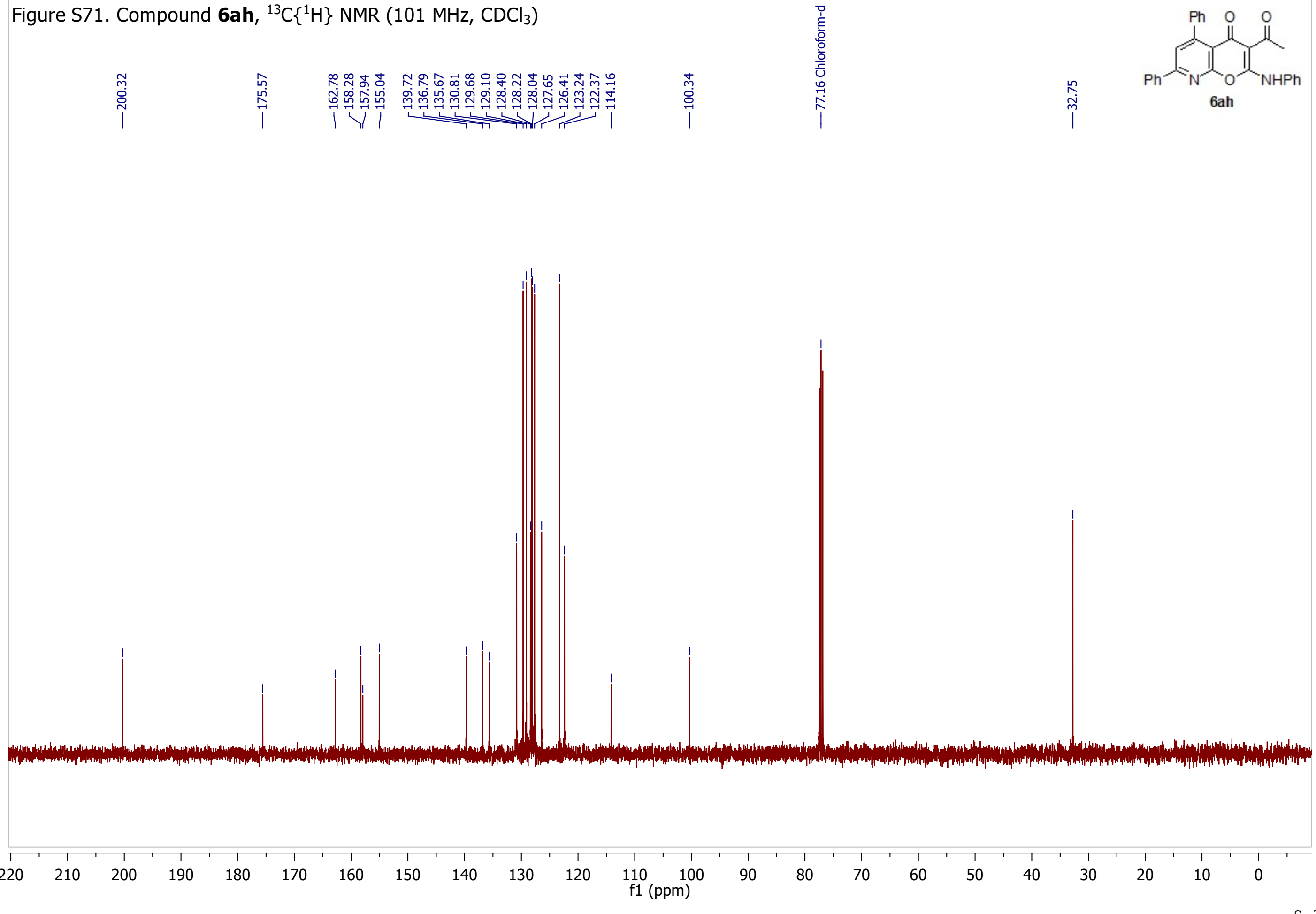

S 72 


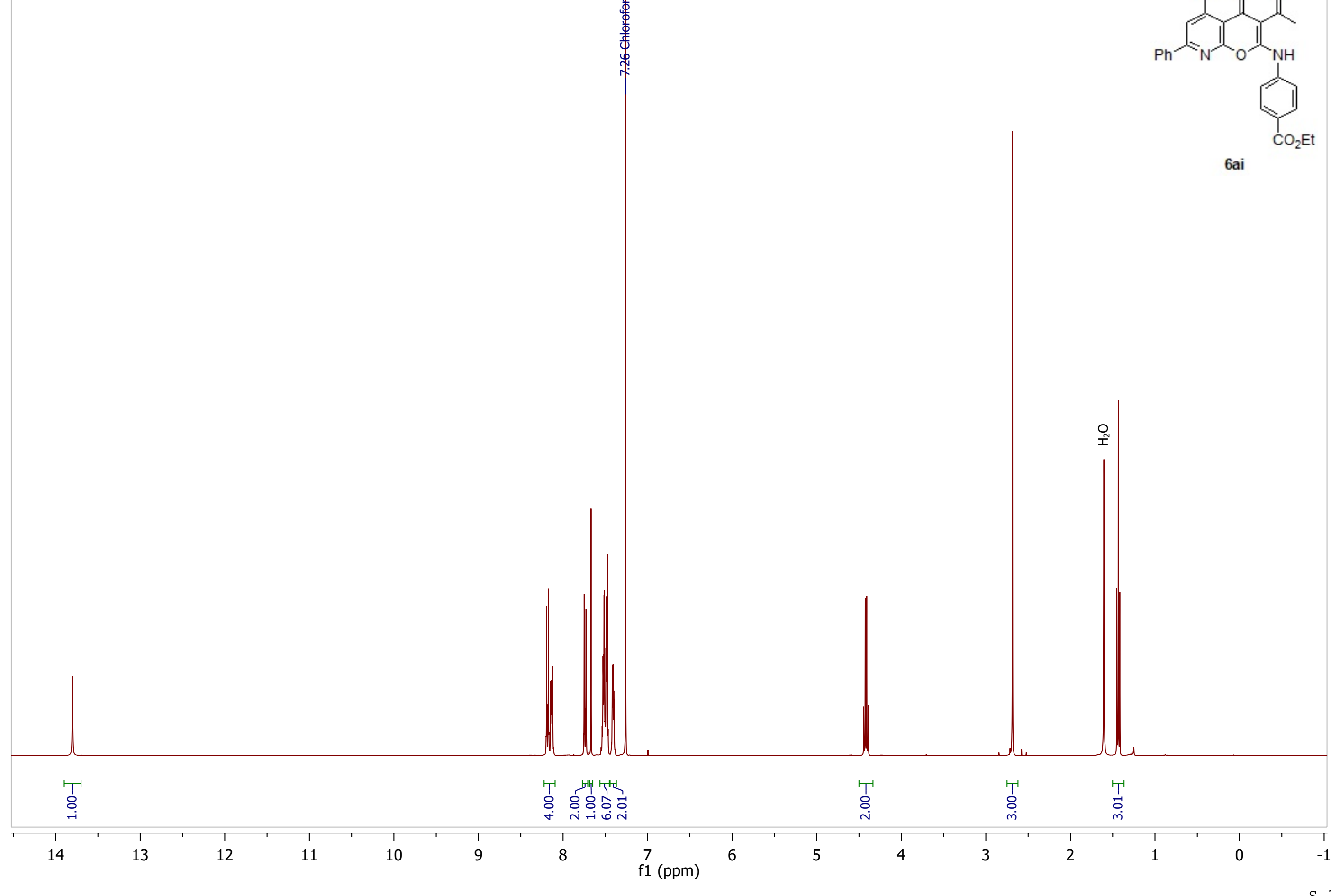


Figure S73. Compound 6ai, ${ }^{13} \mathrm{C}\left\{{ }^{1} \mathrm{H}\right\}$ NMR $\left(101 \mathrm{MHz}, \mathrm{CDCl}_{3}\right)$

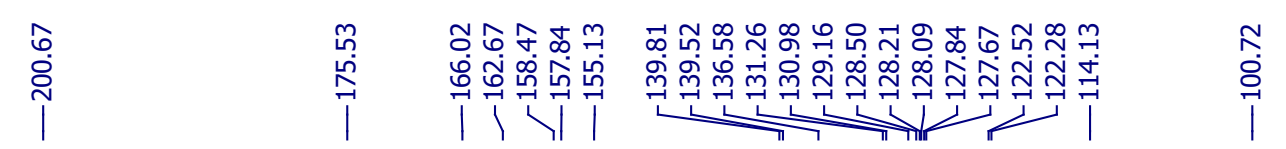

i

$\underset{\substack{0 \\ i}}{1}$

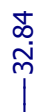

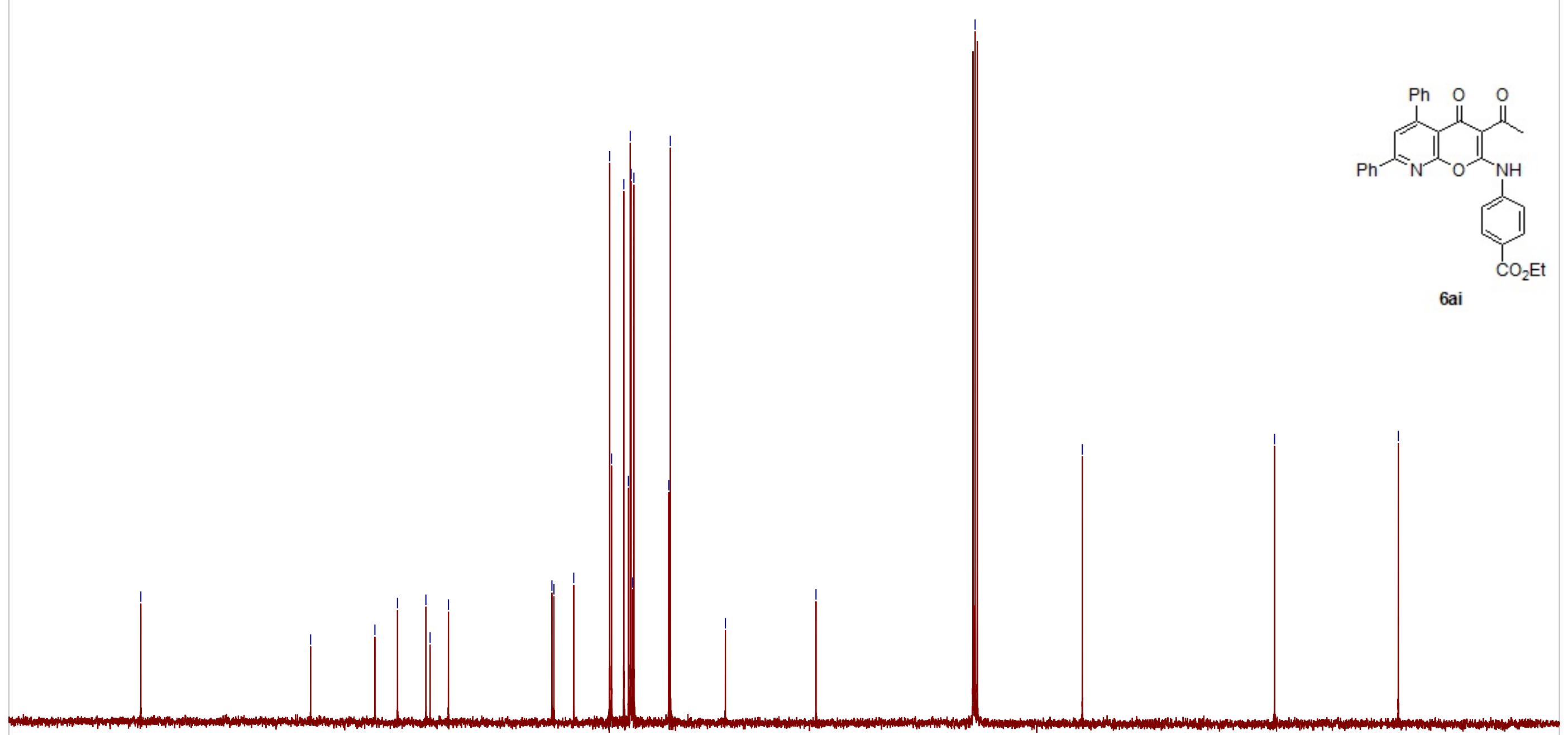


Figure S74. Compound 6aj, ${ }^{1} \mathrm{H}$ NMR $\left(500 \mathrm{MHz} \mathrm{CDCl}_{3}\right)$
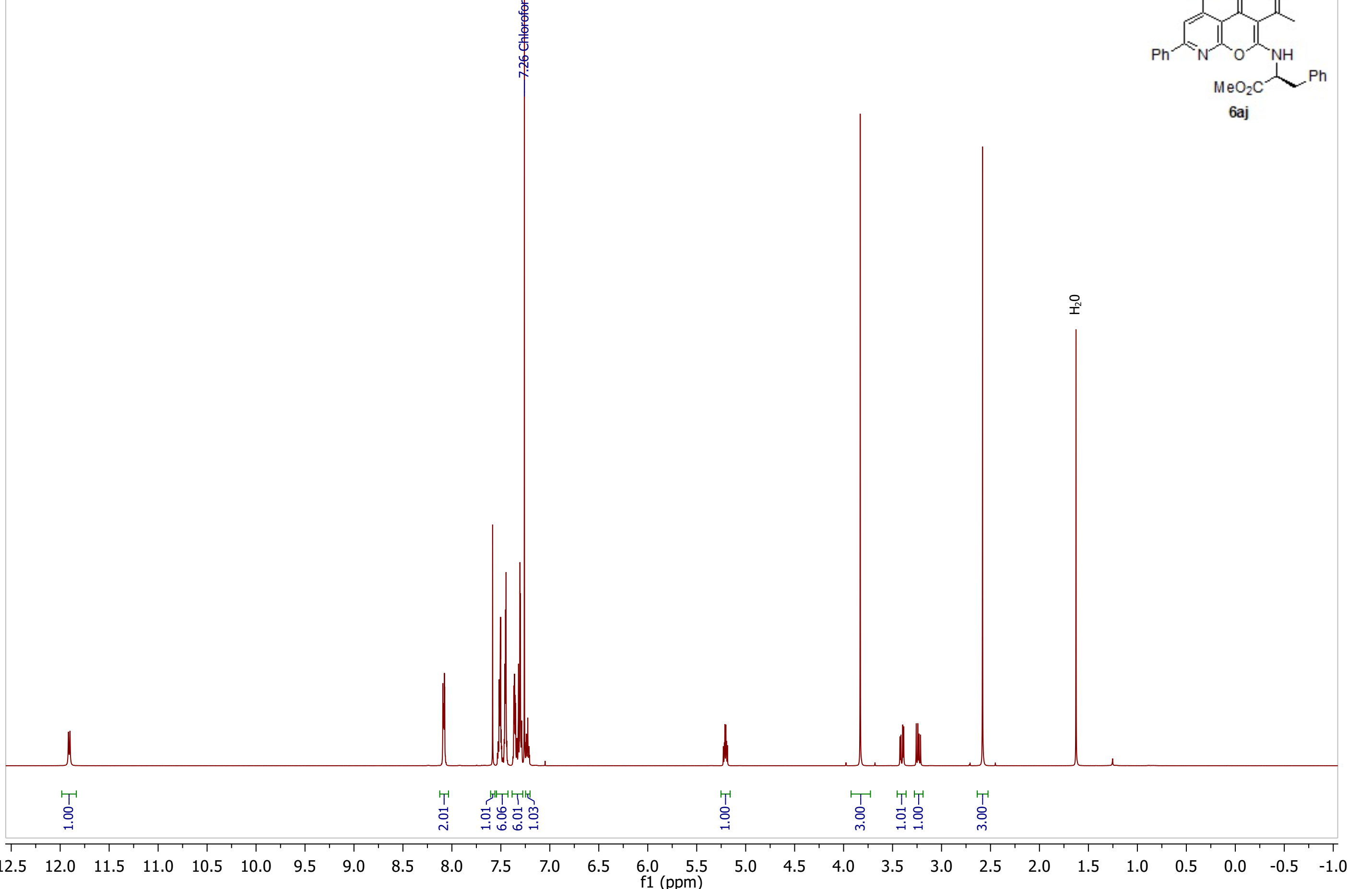
Figure S75. Compound $\mathbf{6 a j},{ }^{13} \mathrm{C}\left\{{ }^{1} \mathrm{H}\right\}$ NMR $\left(126 \mathrm{MHz}, \mathrm{CDCl}_{3}\right)$

|

లై 붕 \&

|

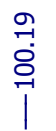

菅

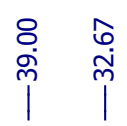

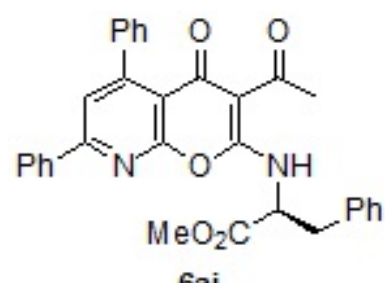

6aj

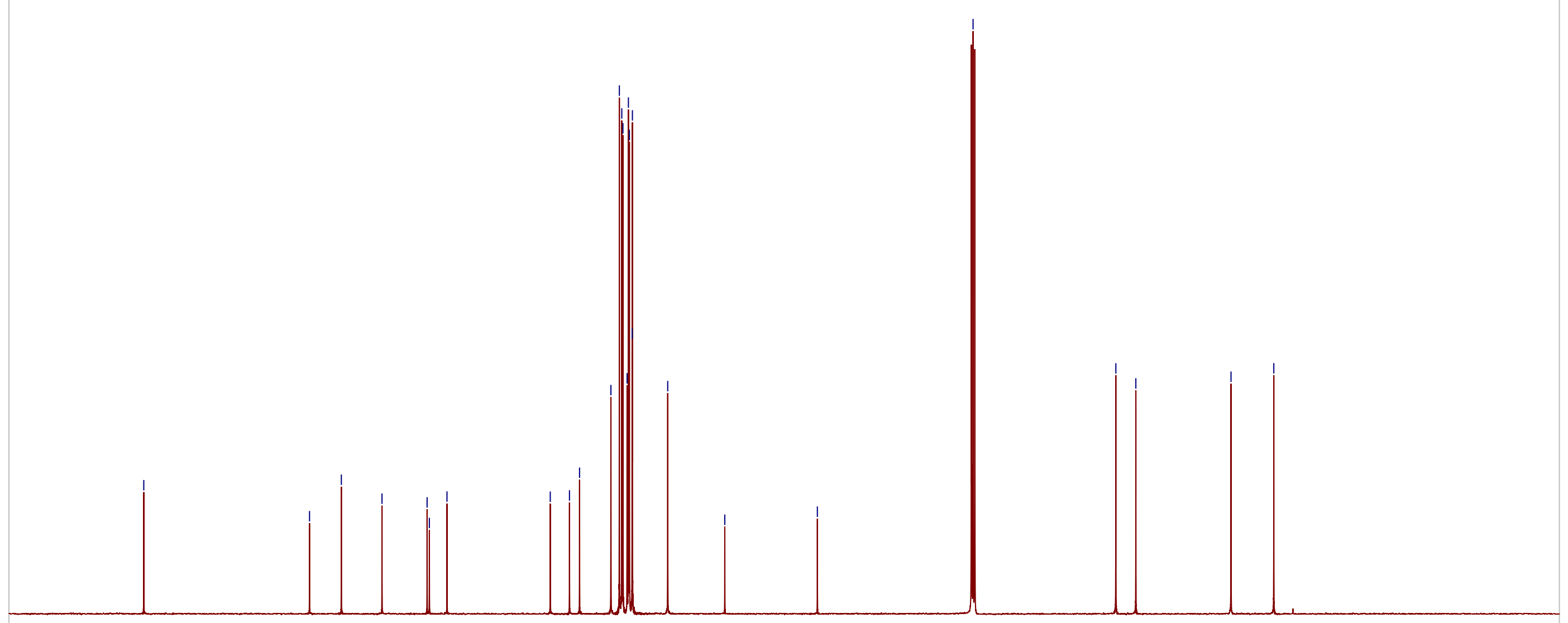



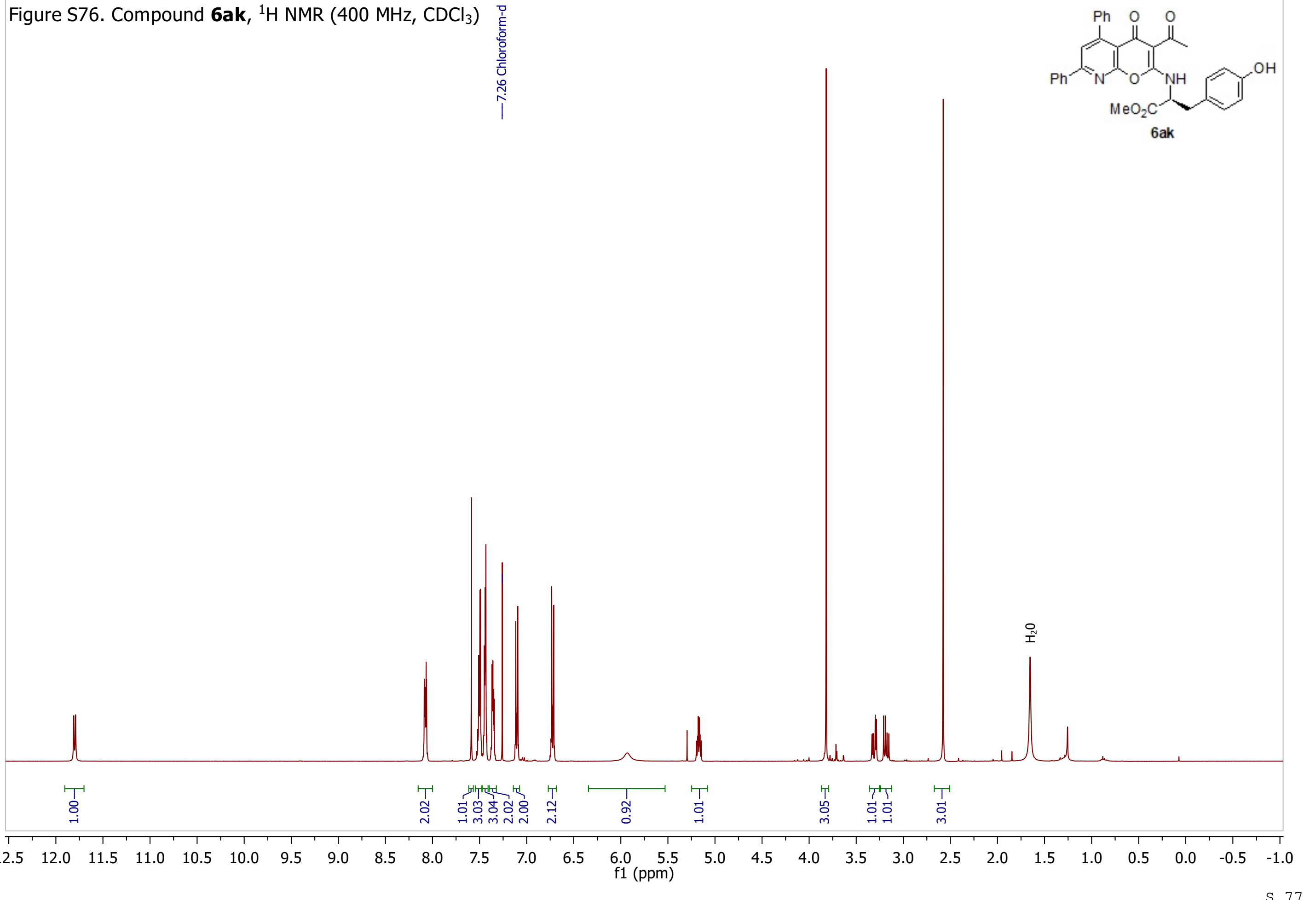
Figure S77. Compound 6ak, ${ }^{13} \mathrm{C}\left\{{ }^{1} \mathrm{H}\right\}$ NMR (101 $\left.\mathrm{MHz}, \mathrm{CDCl}_{3}\right)$

$$
\text { กั่ }
$$

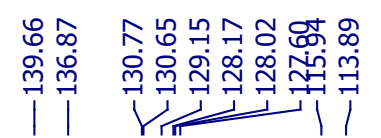

ลั.
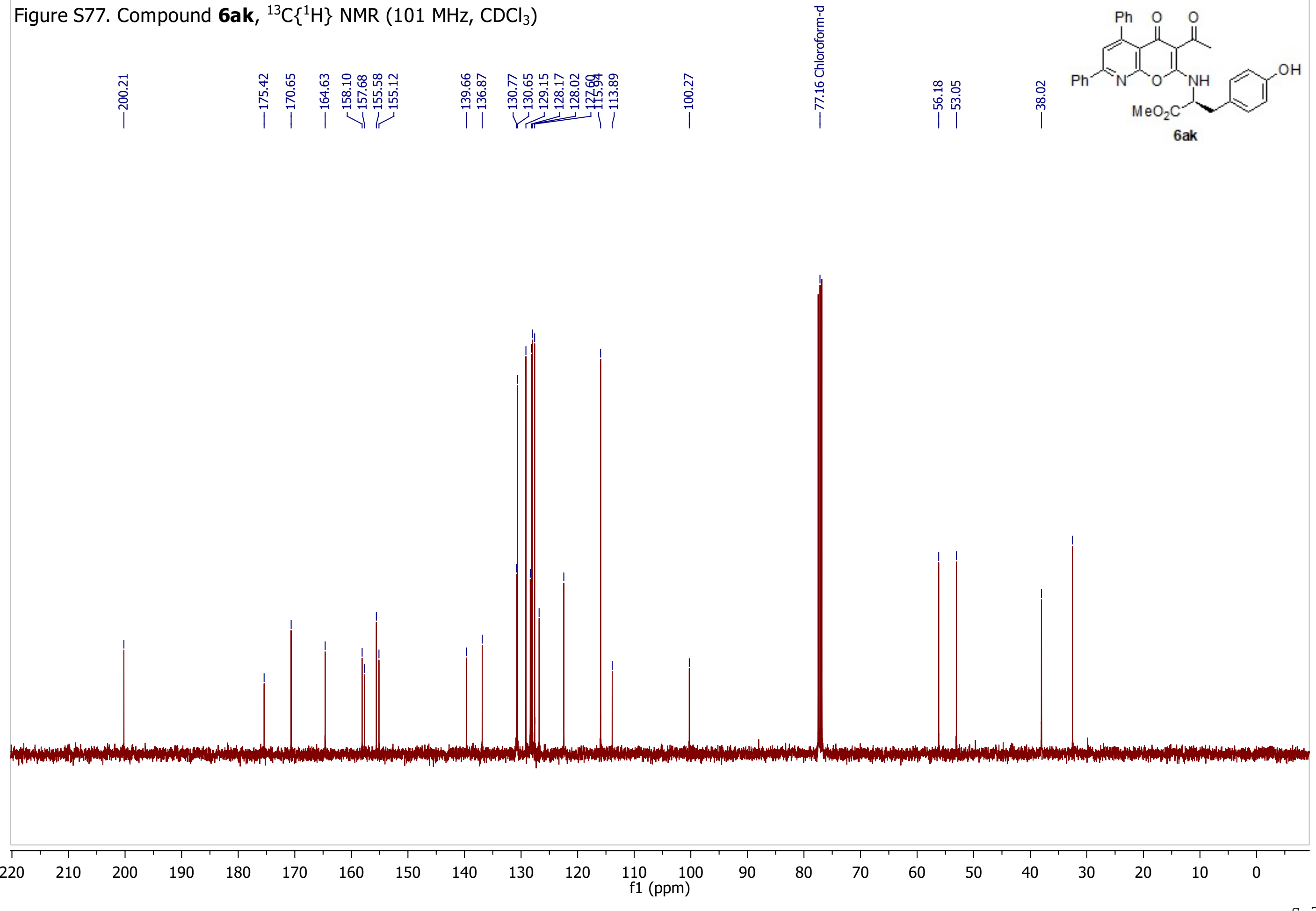

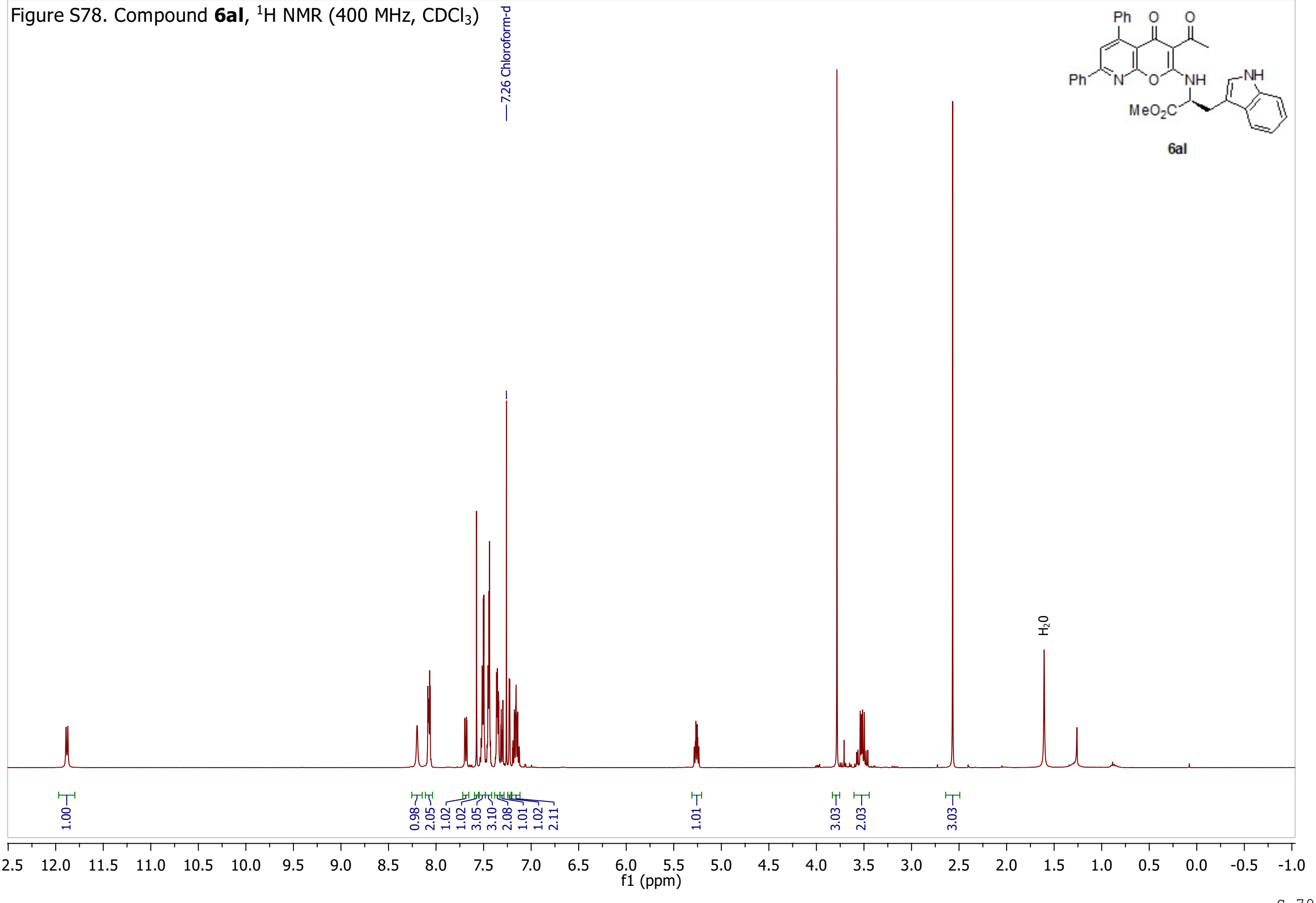
Figure S79. Compound $\mathbf{6 a l},{ }^{13} \mathrm{C}\left\{{ }^{1} \mathrm{H}\right\}$ NMR $\left(101 \mathrm{MHz}, \mathrm{CDCl}_{3}\right)$

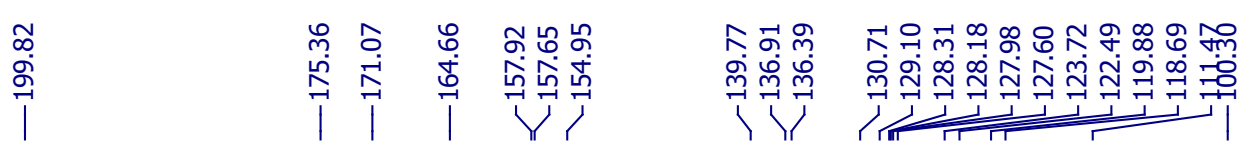

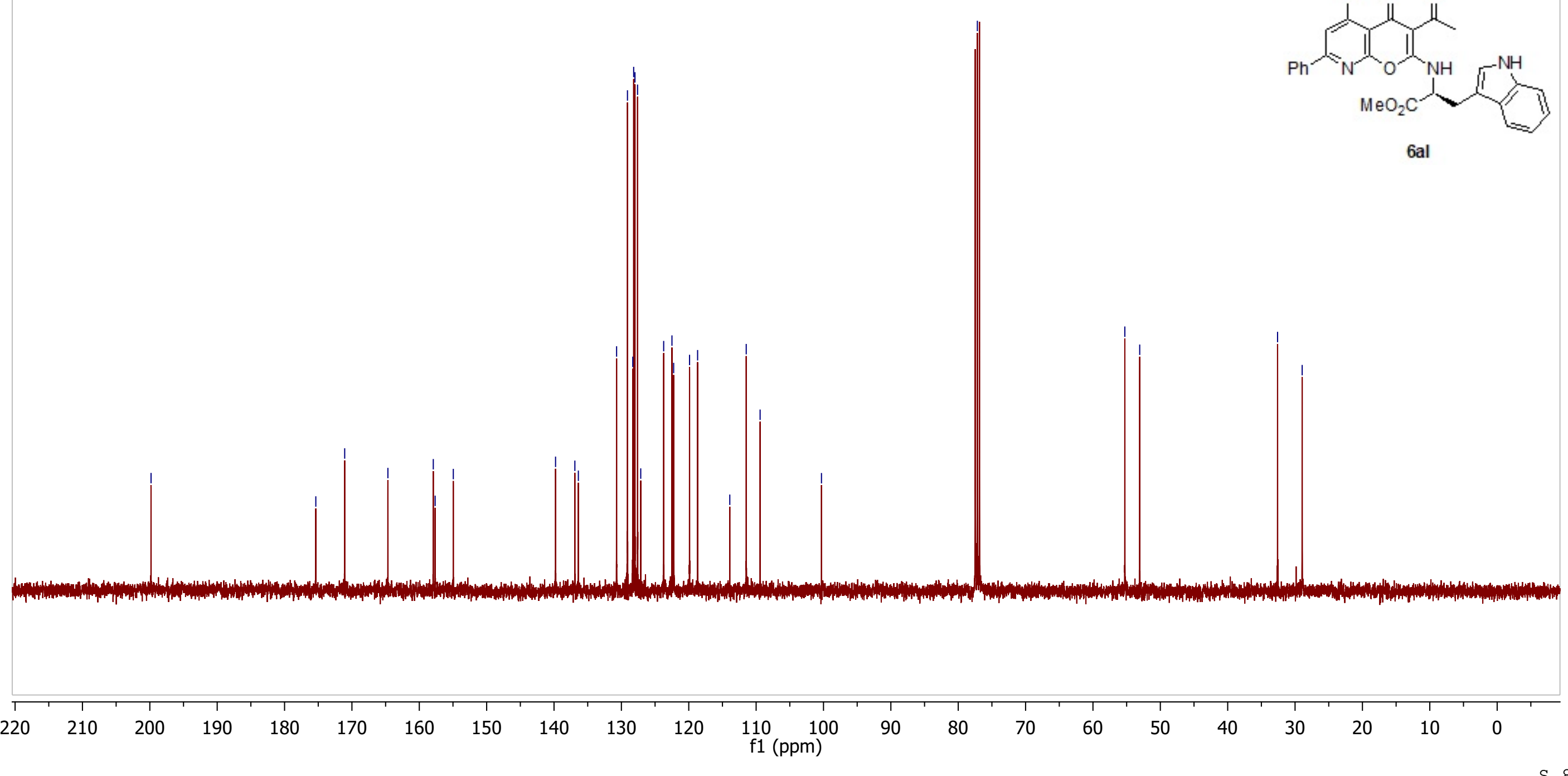


Figure S80. Compound 6am, ${ }^{1} \mathrm{H}$ NMR $\left(400 \mathrm{MHz}, \mathrm{CDCl}_{3}\right)$

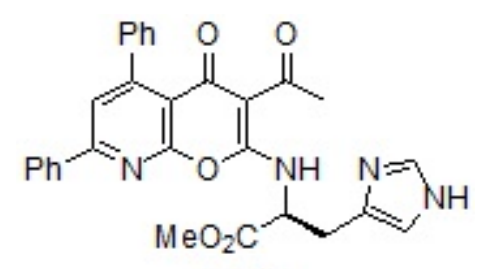

$6 \mathrm{am}$

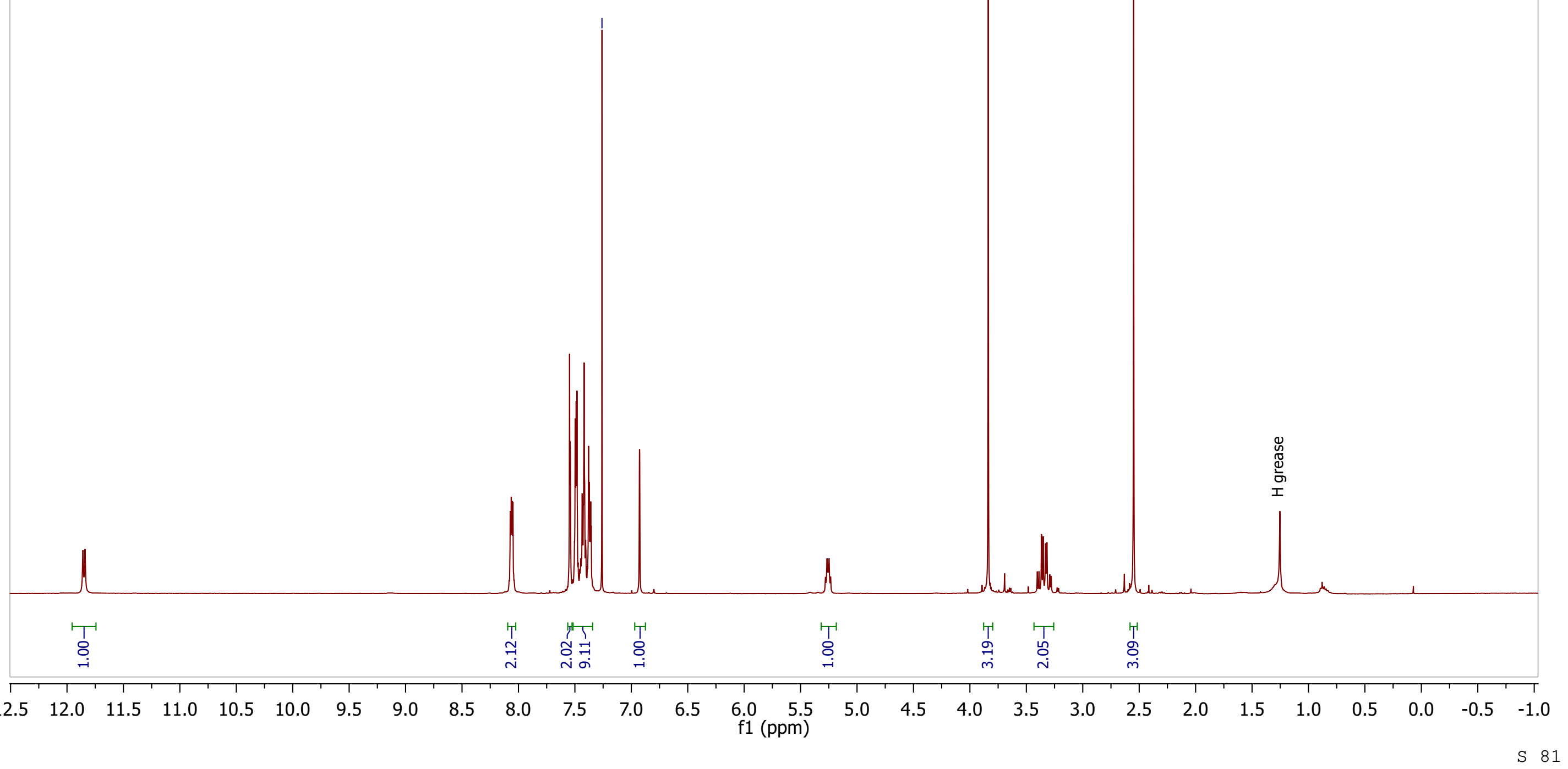


Figure S81. Compound 6am, ${ }^{13} \mathrm{C}\left\{{ }^{1} \mathrm{H}\right\}$ NMR (151 MHz, CDCl 3 )

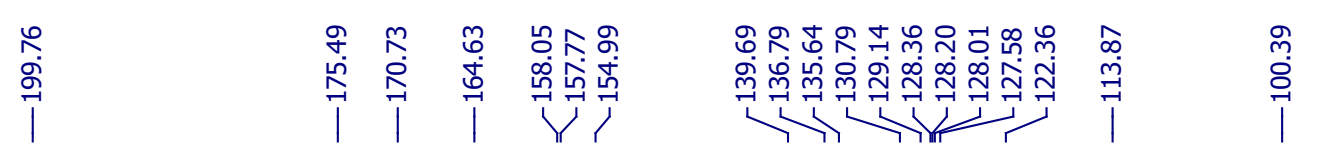

范

เึ่

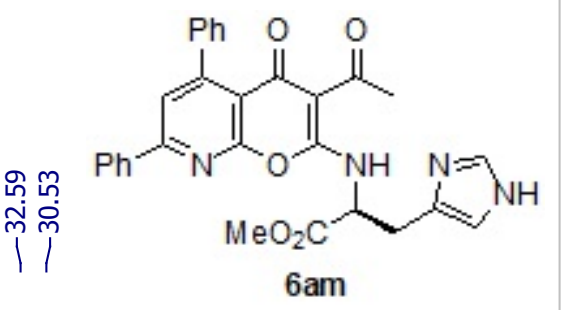

6 am

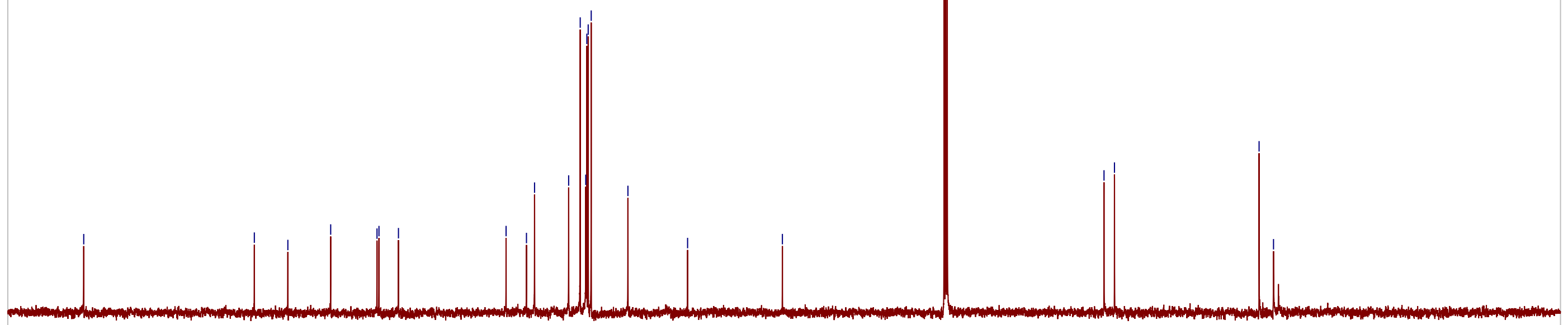


Figure S82. Compound 6ba, ${ }^{1} \mathrm{H}$ NMR (400 MHz, $\left.\mathrm{CDCl}_{3}\right)$

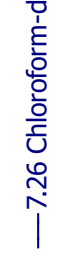

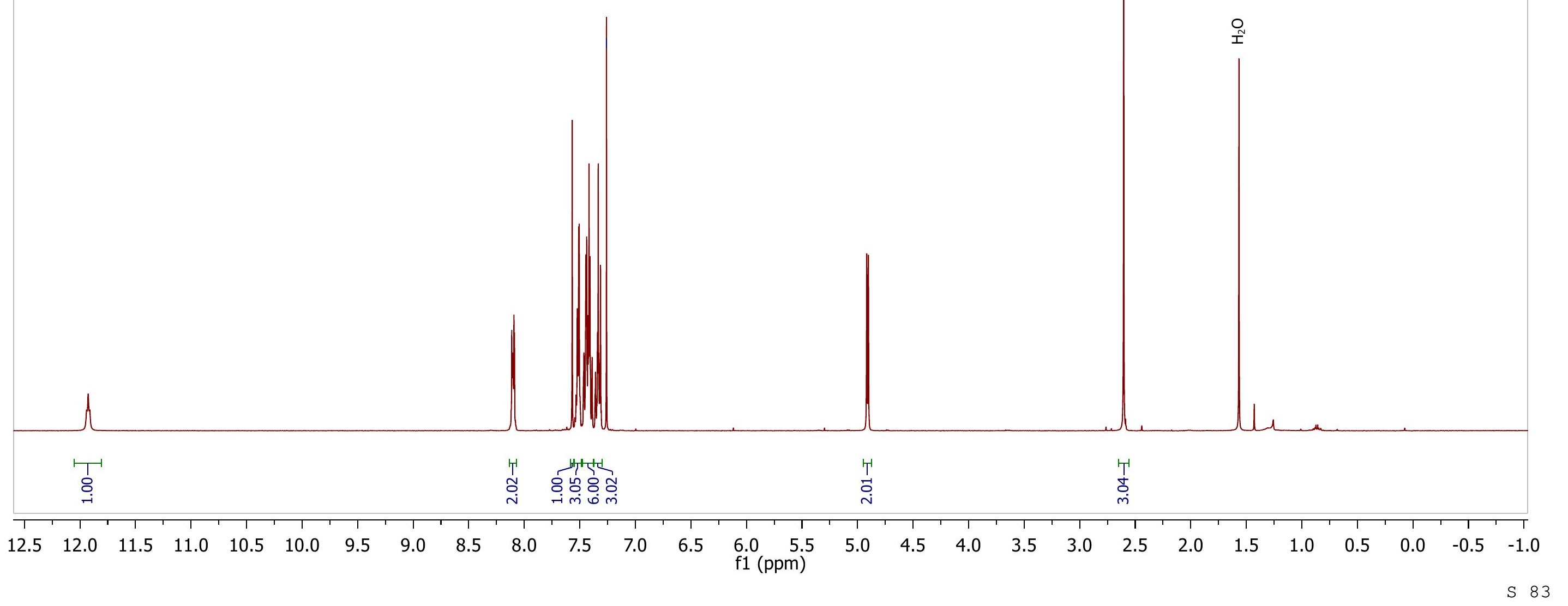


Figure S83. Compound $\mathbf{6 b a},{ }^{13} \mathrm{C}\left\{{ }^{1} \mathrm{H}\right\}$ NMR $\left(101 \mathrm{MHz}, \mathrm{CDCl}_{3}\right)$

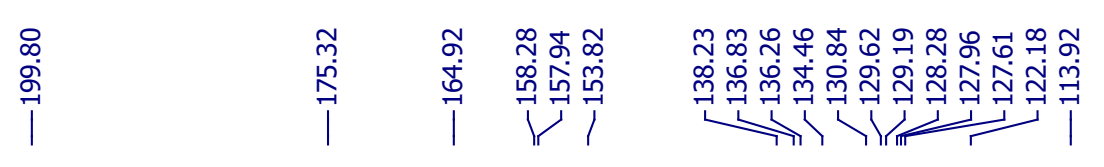

눙

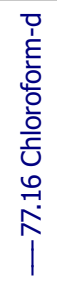<smiles>CC(=O)c1c(NCc2ccccc2)oc2nc(-c3ccccc3)cc(-c3ccc(Cl)cc3)c2c1=O</smiles>

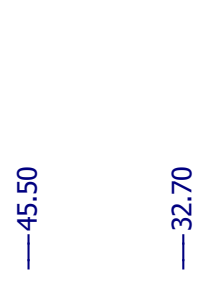

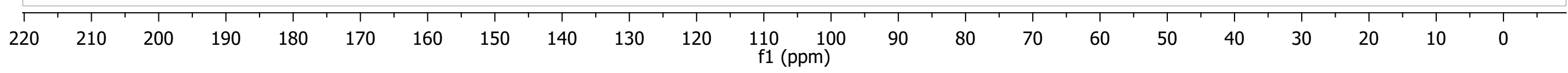

S 84 
Figure S84. Compound 6bb, ${ }^{1} \mathrm{H}$ NMR $\left(400 \mathrm{MHz} \mathrm{CDCl}_{3}\right)$

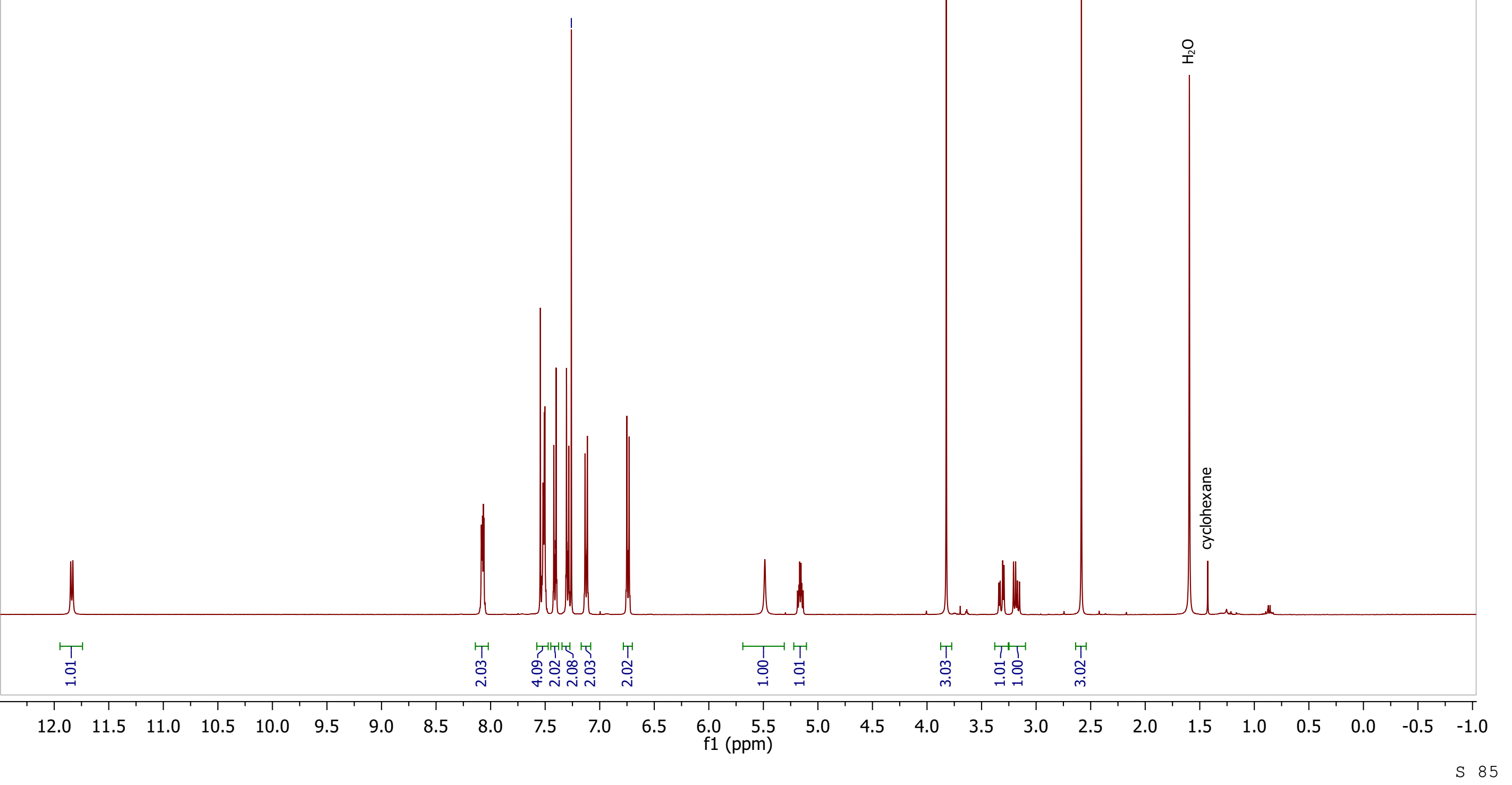




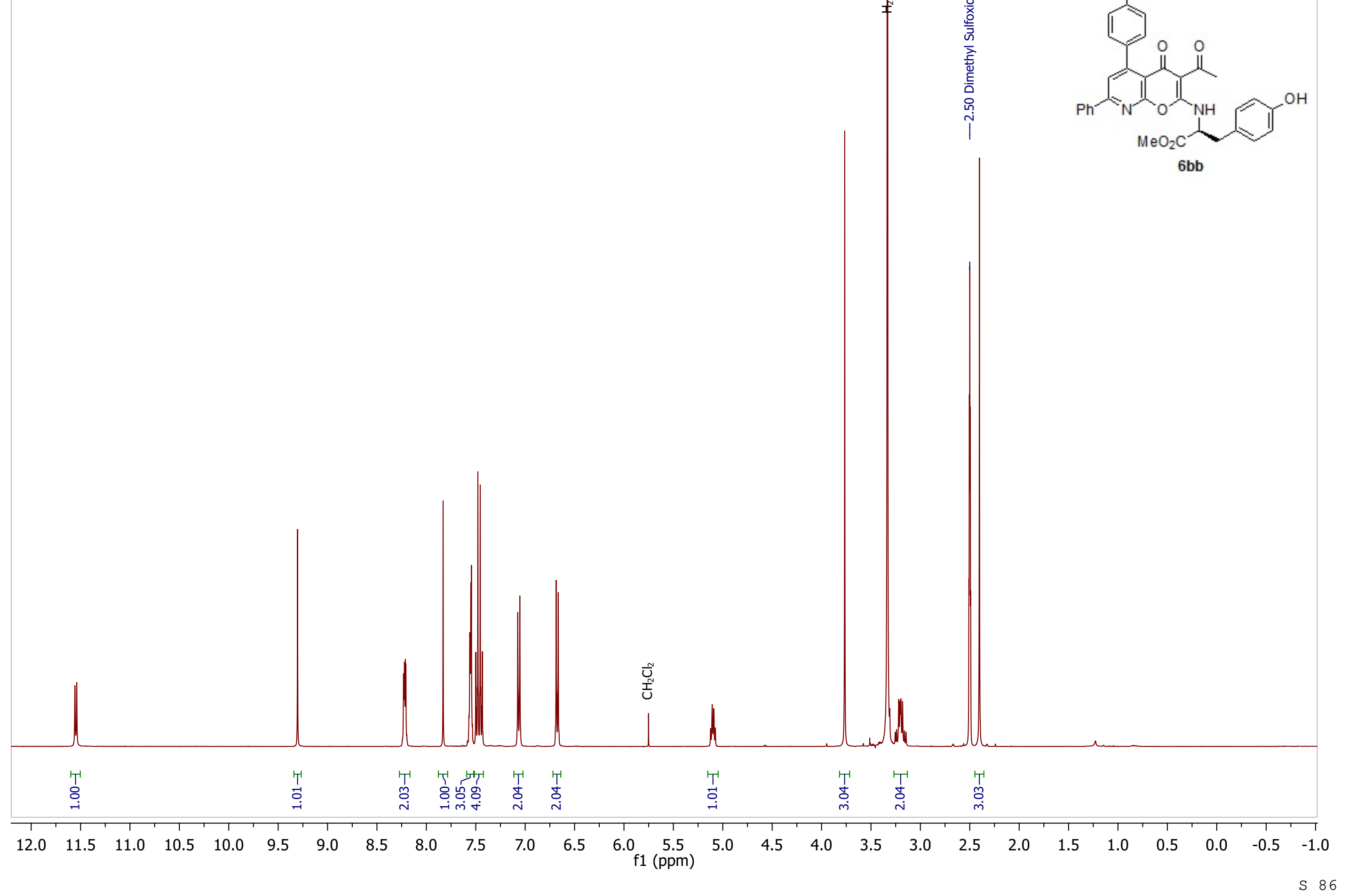



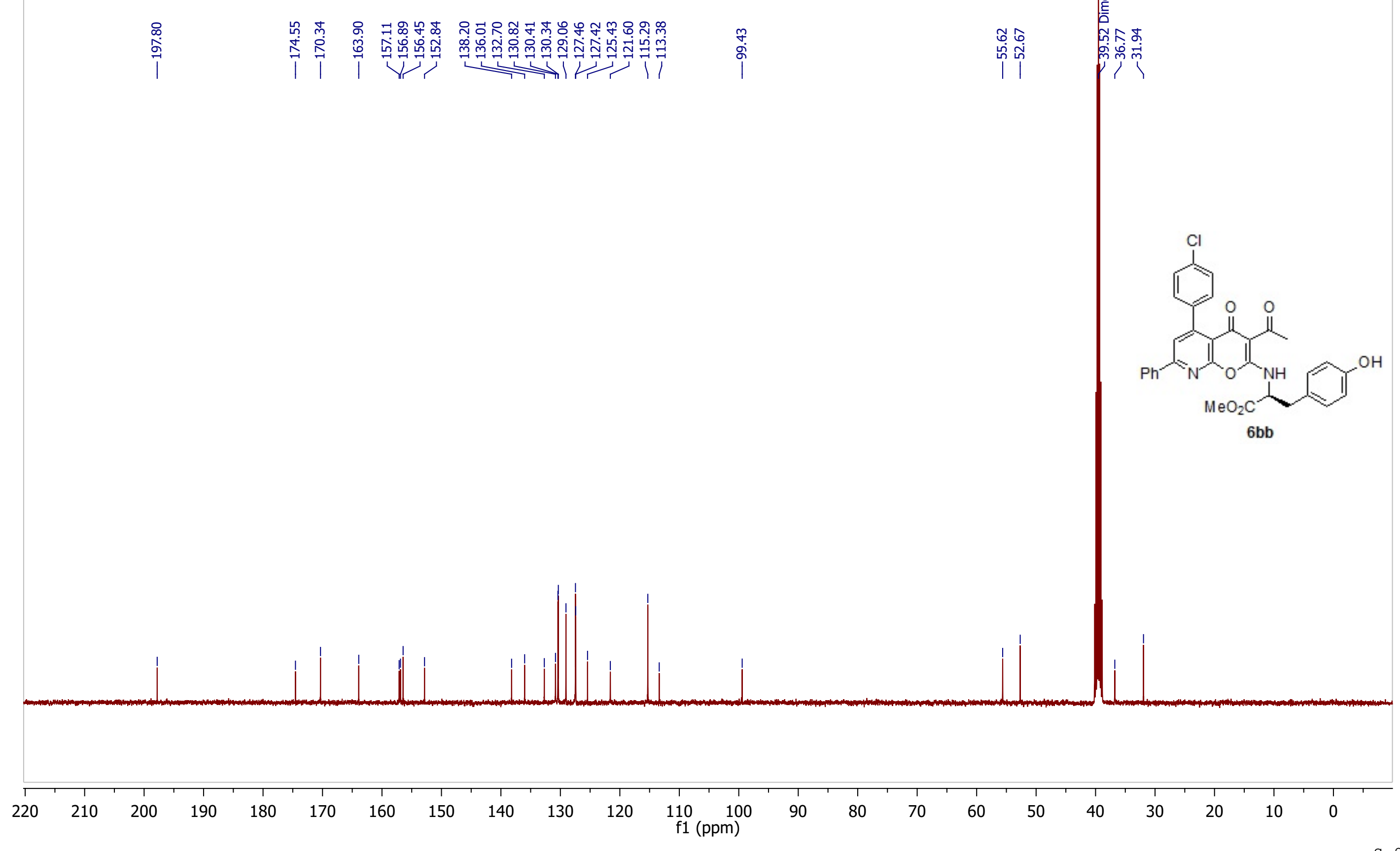
Figure S87. Compound 6bc, ${ }^{1} \mathrm{H}$ NMR (400 MHz, $\left.\mathrm{CDCl}_{3}\right)$

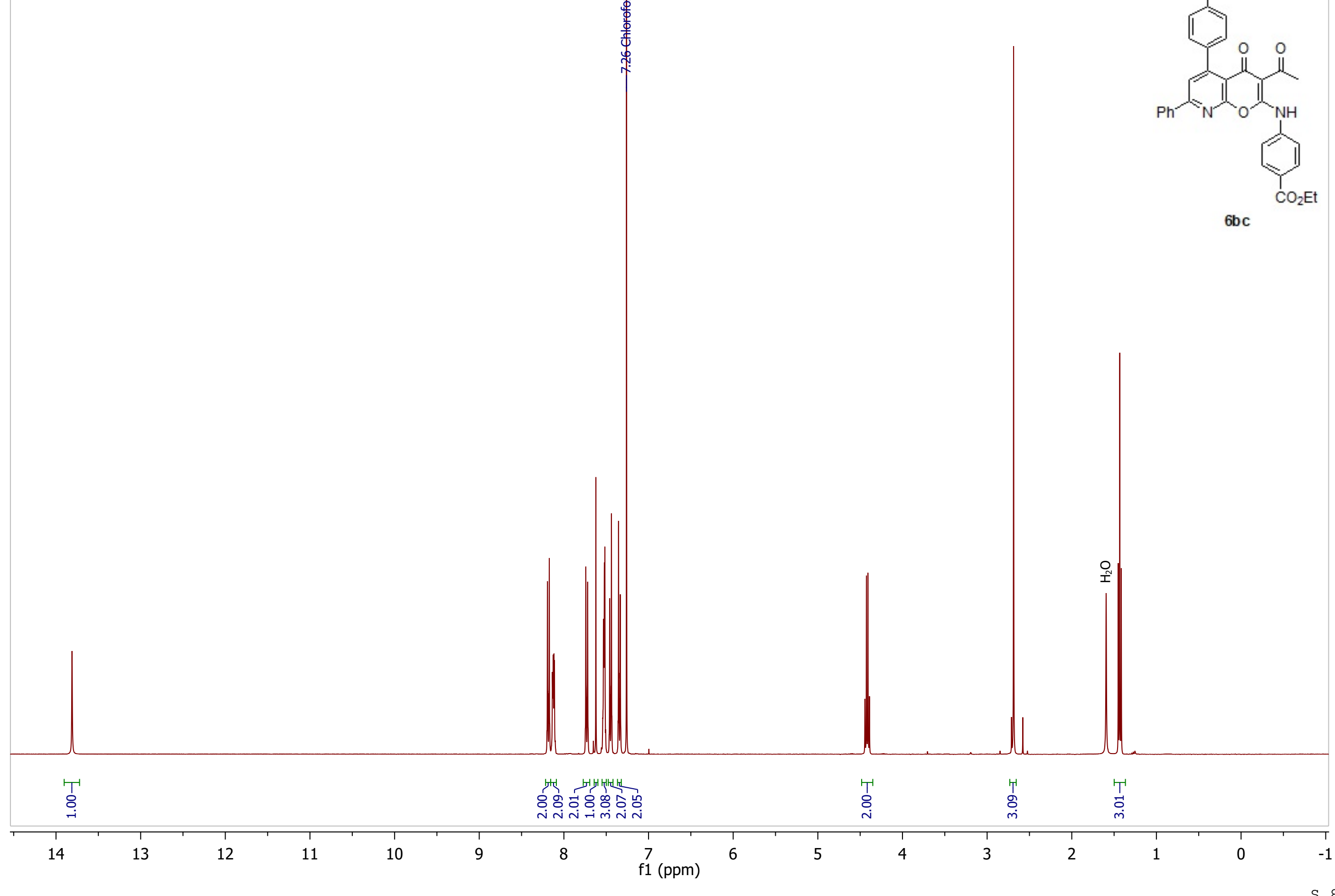


Figure S88. Compound 6bc, ${ }^{13} \mathrm{C}\left\{{ }^{1} \mathrm{H}\right\}$ NMR $\left(101 \mathrm{MHz}, \mathrm{CDCl}_{3}\right)$

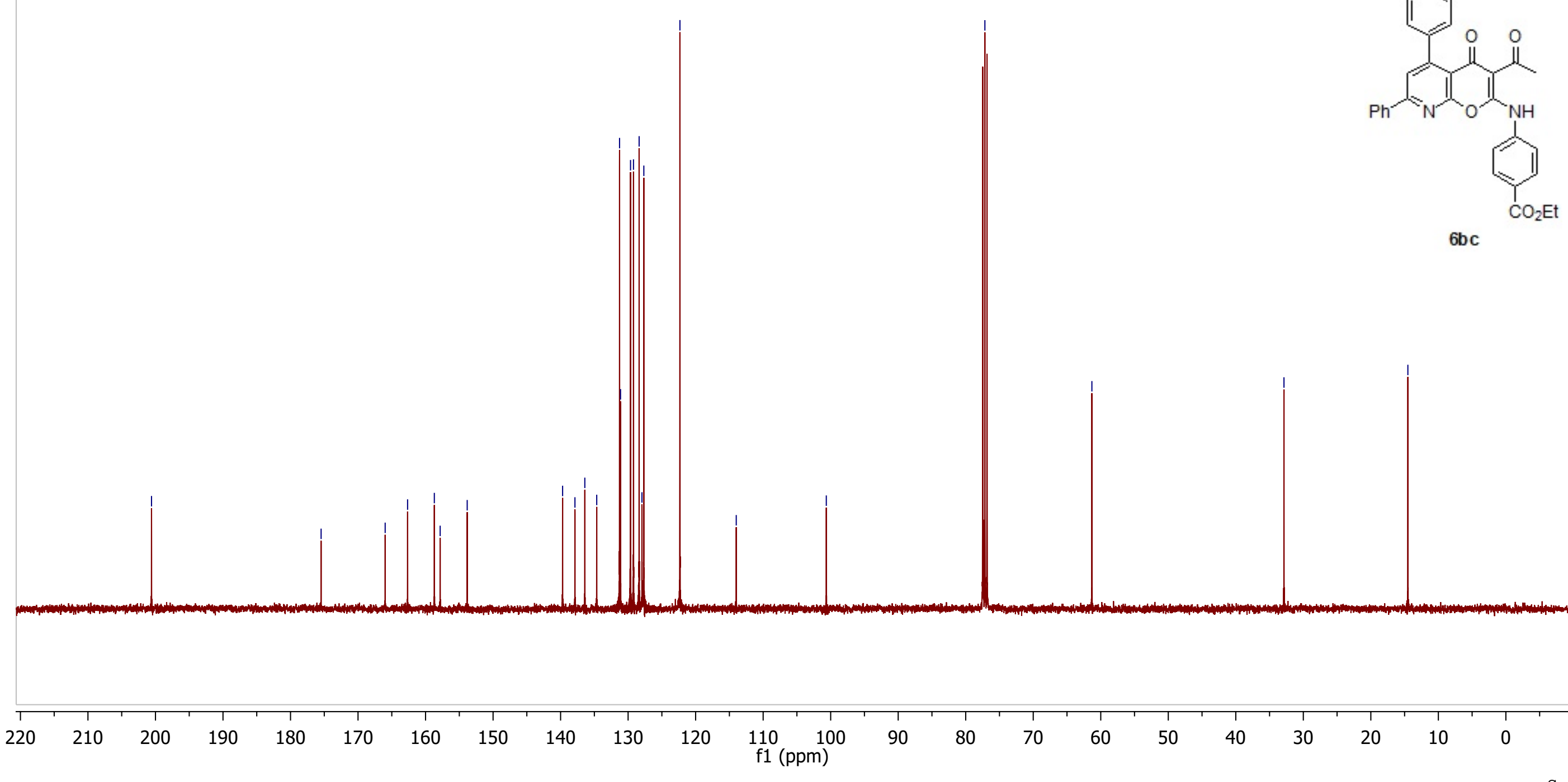


Figure S89. Compound 6ca, ${ }^{1} \mathrm{H}$ NMR $\left(400 \mathrm{MHz} \mathrm{CDCl}_{3}\right)$
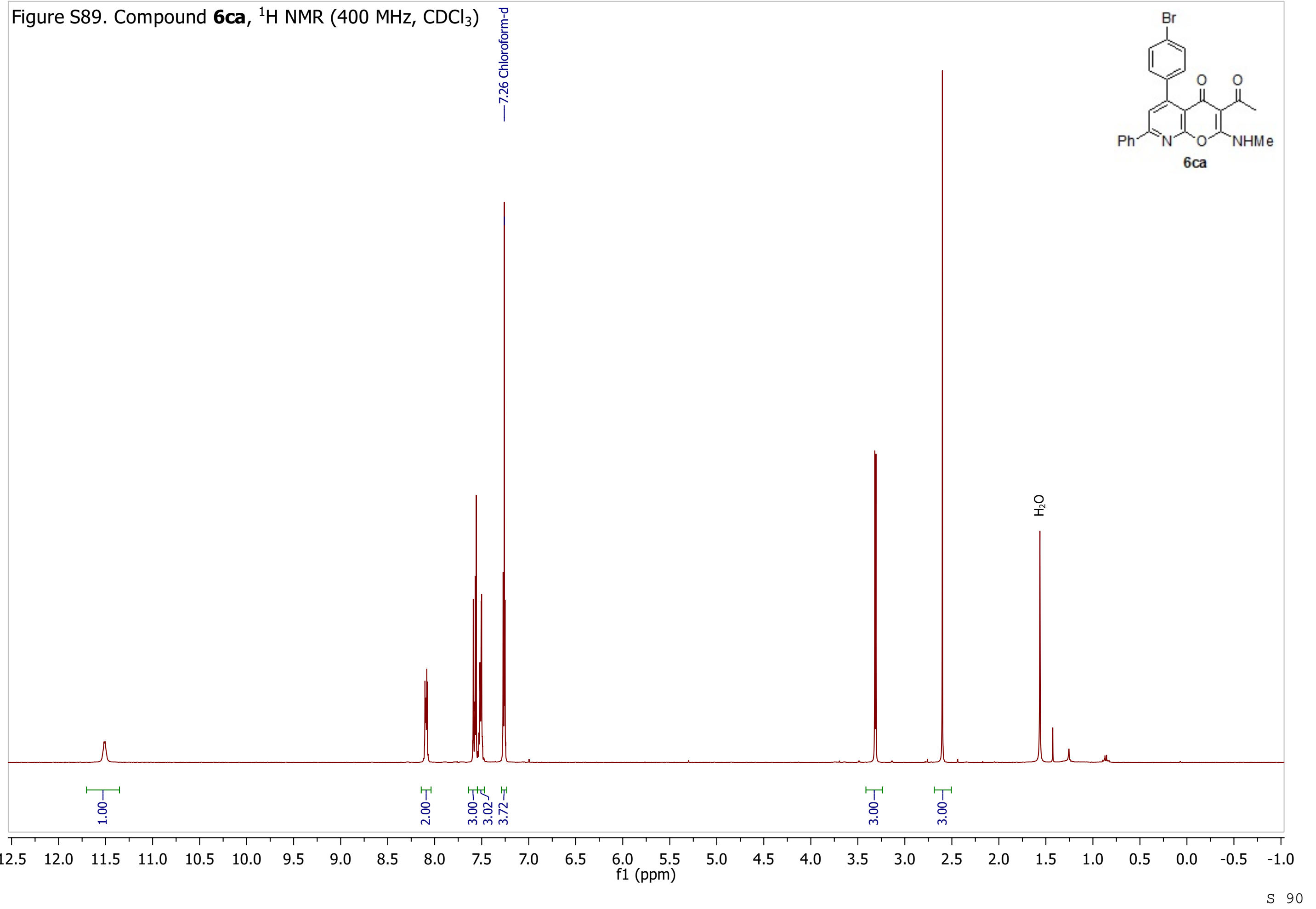
Figure S90. Compound $\mathbf{6} \mathbf{c a},{ }^{13} \mathrm{C}\left\{{ }^{1} \mathrm{H}\right\}$ NMR $\left(101 \mathrm{MHz}, \mathrm{CDCl}_{3}\right)$

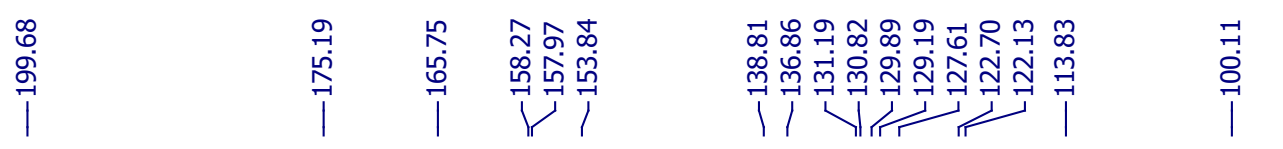

능 농

๗ָं
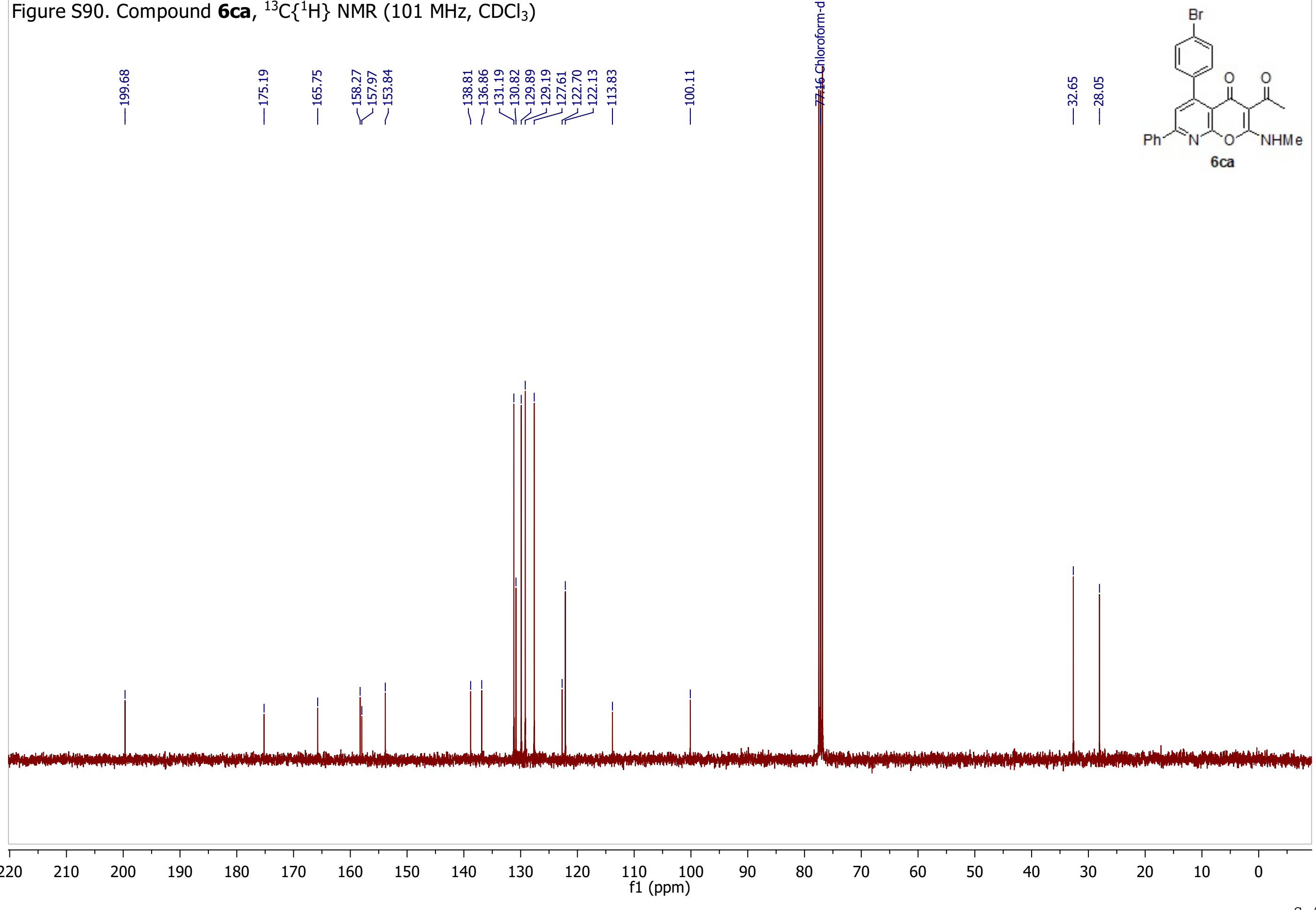
Figure S91. Compound 6cb, ${ }^{1} \mathrm{H}$ NMR (400 MHz, $\mathrm{CDCl}_{3}$ )
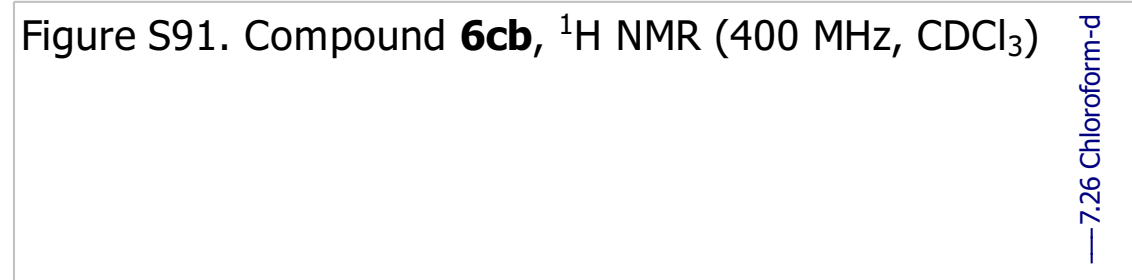

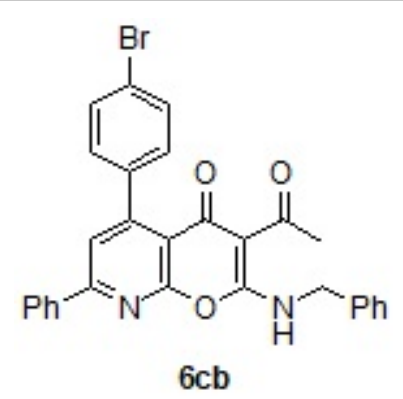

옴

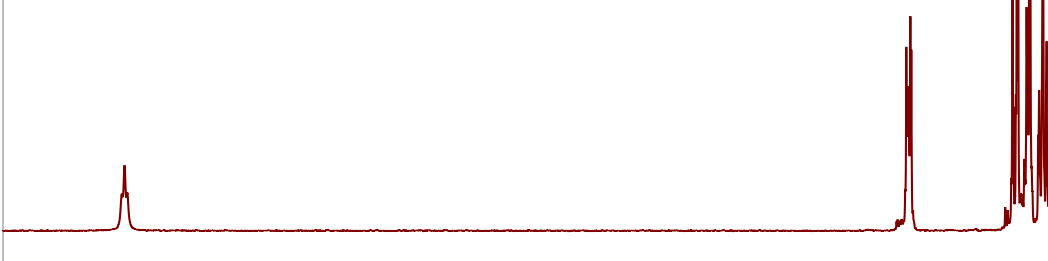

T) món

mं

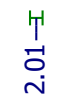

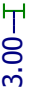

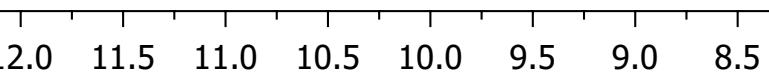

8.0

7.5 
Figure S92. Compound $\mathbf{6 c b},{ }^{13} \mathrm{C}\left\{{ }^{1} \mathrm{H}\right\}$ NMR $\left(101 \mathrm{MHz}, \mathrm{CDCl}_{3}\right)$

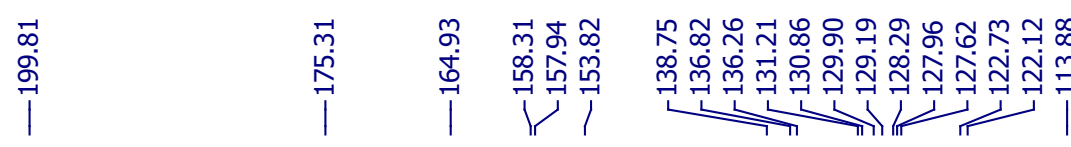

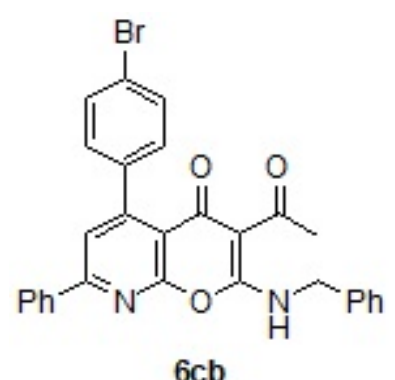

$6 \mathrm{cb}$

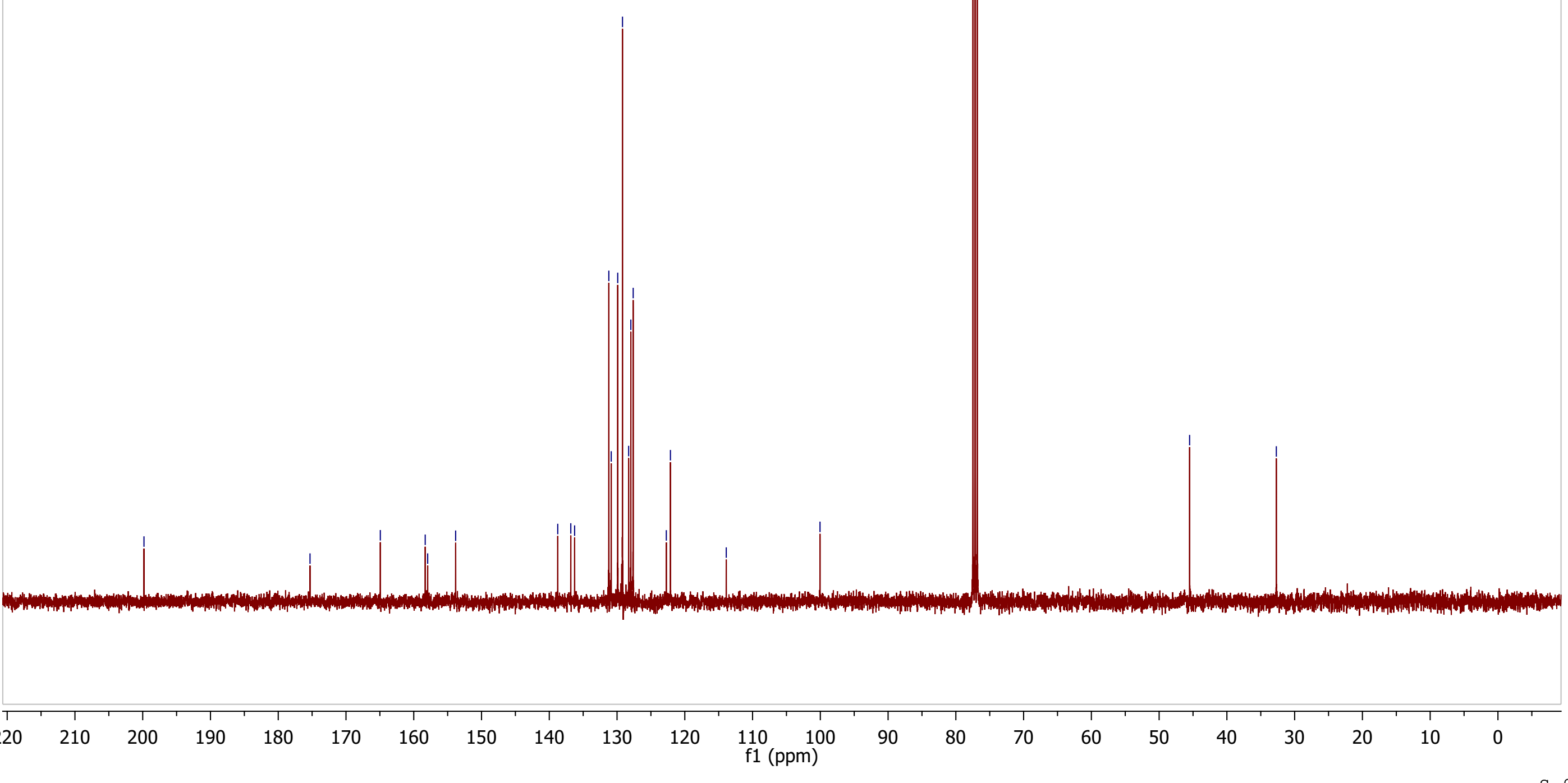


Figure S93. Compound $6 \mathrm{cc}^{1}{ }^{1} \mathrm{H} \mathrm{NMR}\left(400 \mathrm{MHz}, \mathrm{CDCl}_{3}\right)$

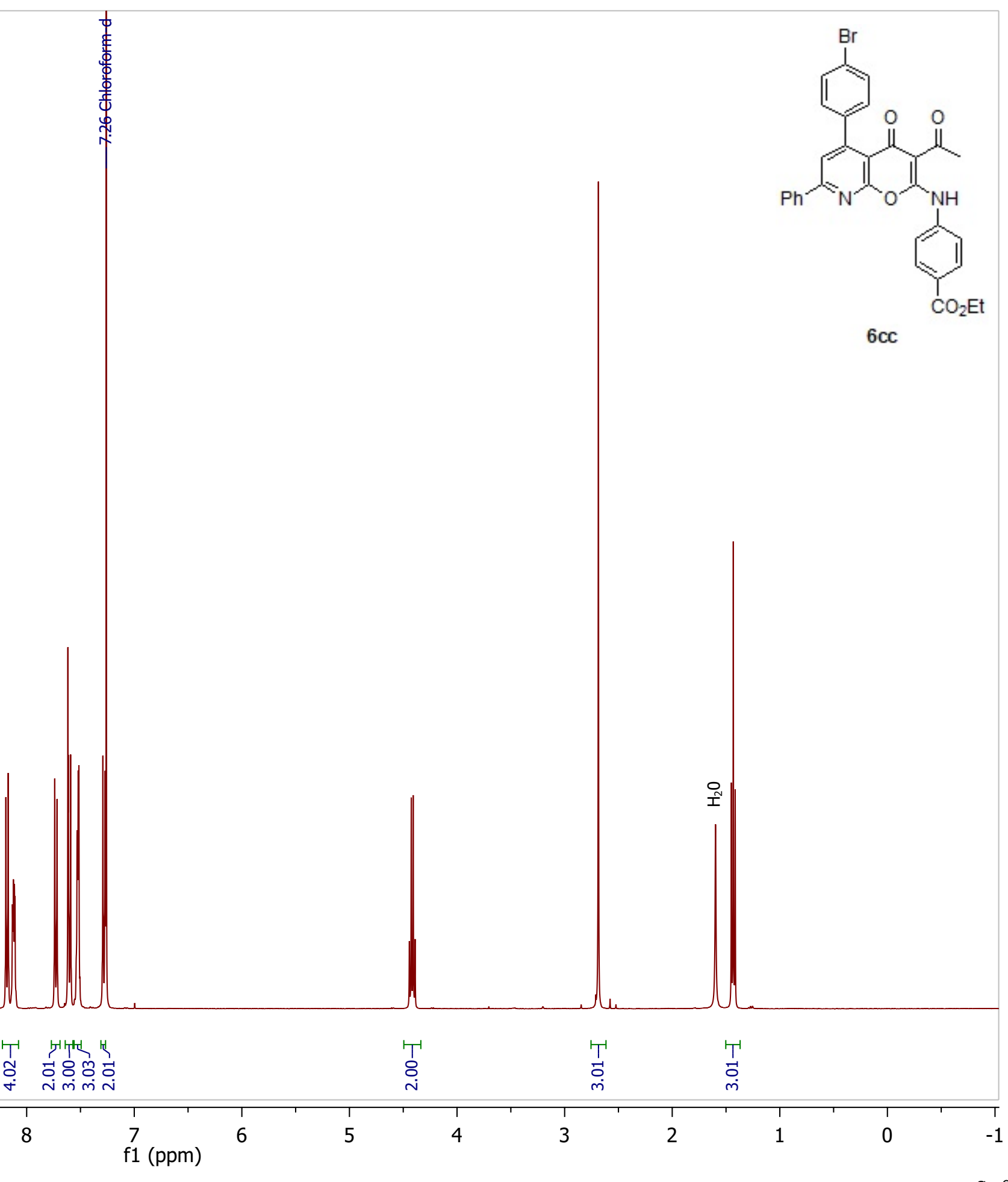


Figure S94. Compound $6 \mathrm{cc},{ }^{13} \mathrm{C}\left\{{ }^{1} \mathrm{H}\right\}$ NMR $\left(101 \mathrm{MHz}, \mathrm{CDCl}_{3}\right)$

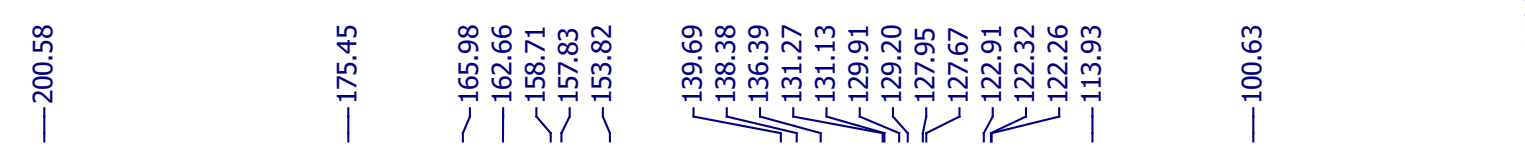

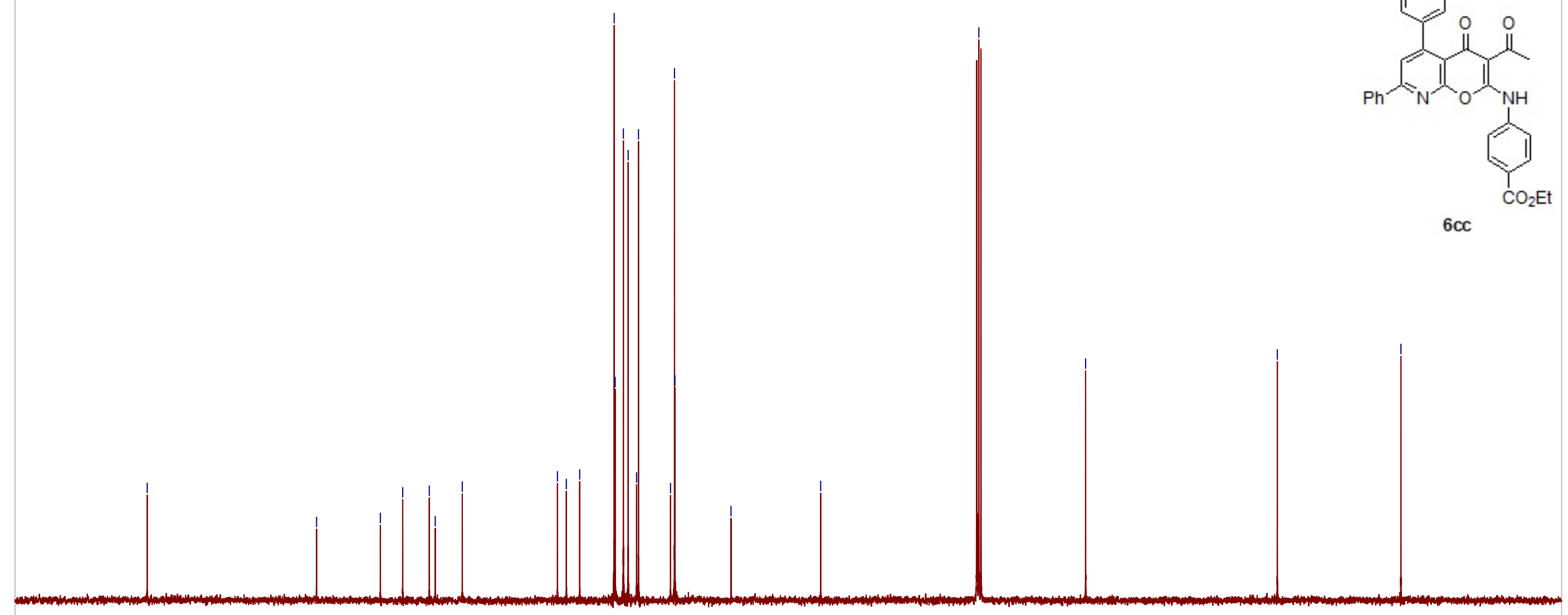

$\begin{array}{rlllllllllllllllllllllllll}220 & 210 & 200 & 190 & 180 & 170 & 160 & 150 & 140 & 130 & 120 & \begin{array}{c}110 \\ \mathrm{f} 1(\mathrm{ppm})\end{array} & 100 & 80 & 70 & 60 & 50 & 40 & 30 & 20 & 10 & 0 & \end{array}$


Figure S95. Compound 6da, ${ }^{1} \mathrm{H}$ NMR $\left(400 \mathrm{MHz} \mathrm{CDCl}_{3}\right)$
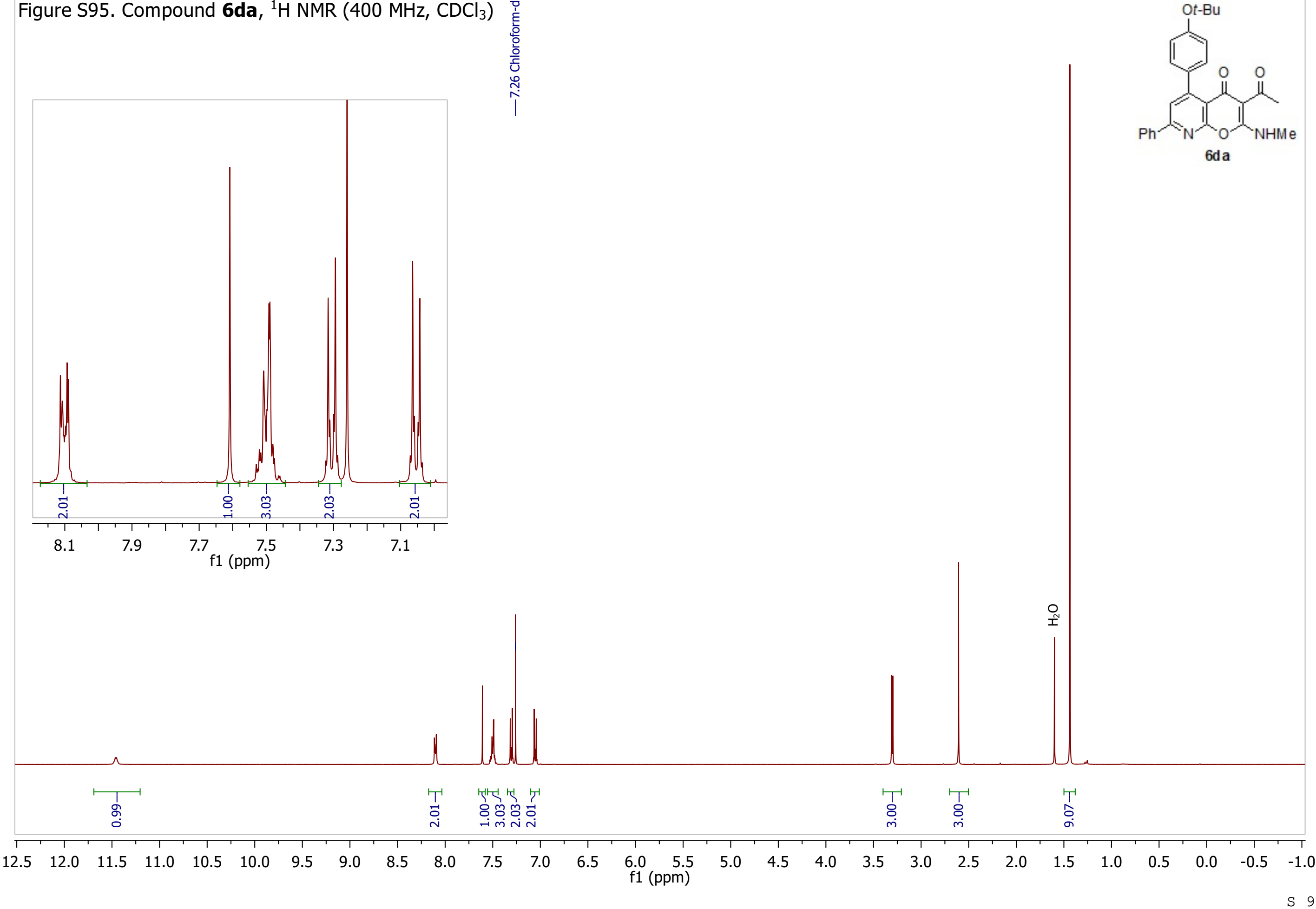

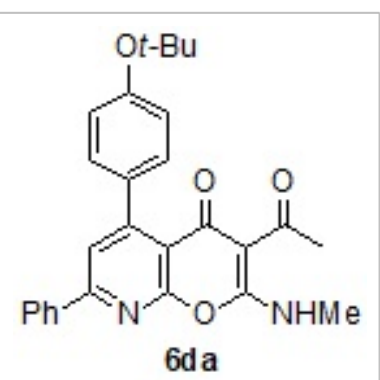

$6 \mathrm{da}$ 

Figure S97. Compound $\mathbf{6 d b},{ }^{1} \mathrm{H}$ NMR $\left(400 \mathrm{MHz}, \mathrm{CDCl}_{3}\right)$

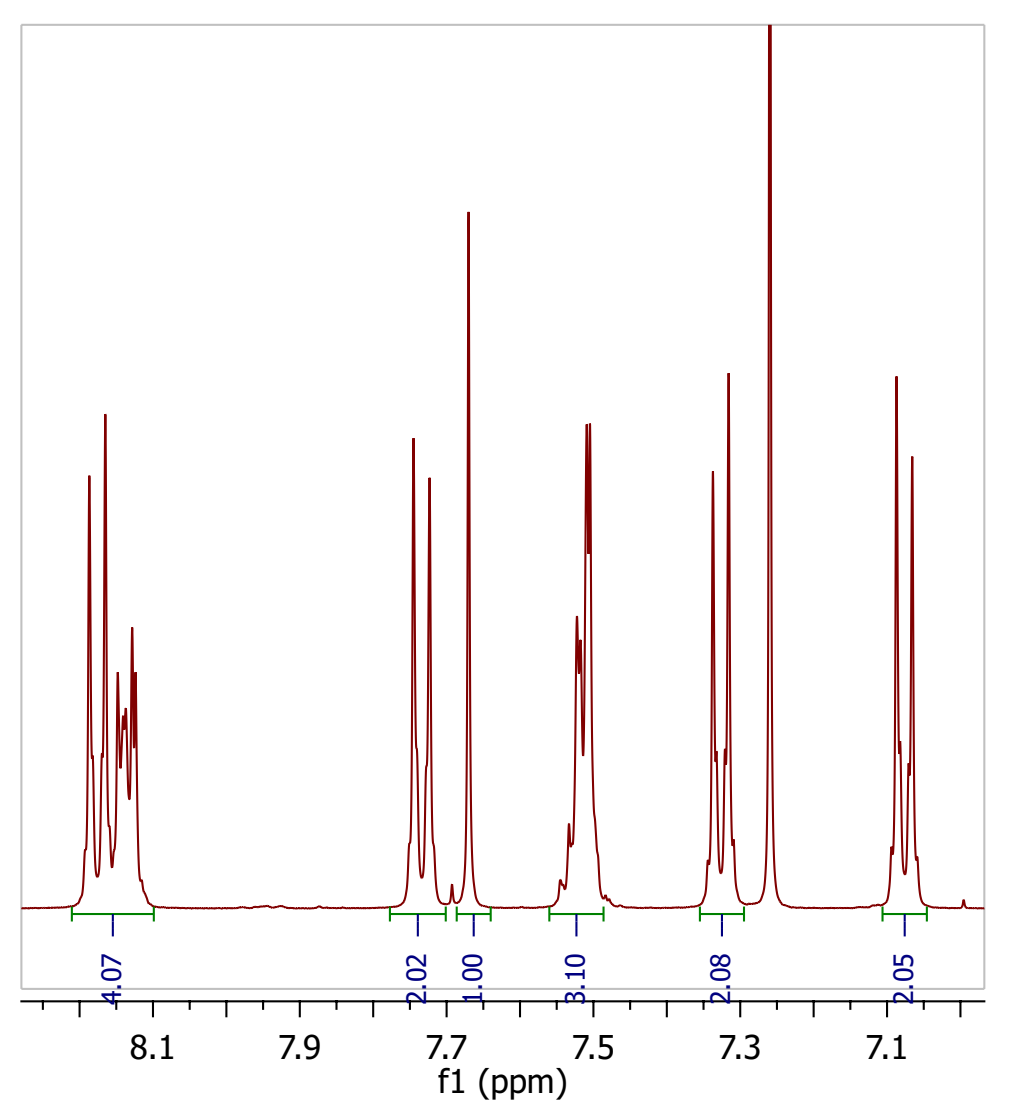

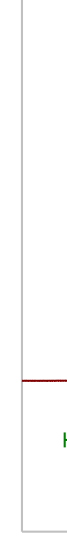

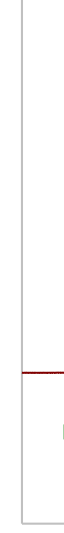

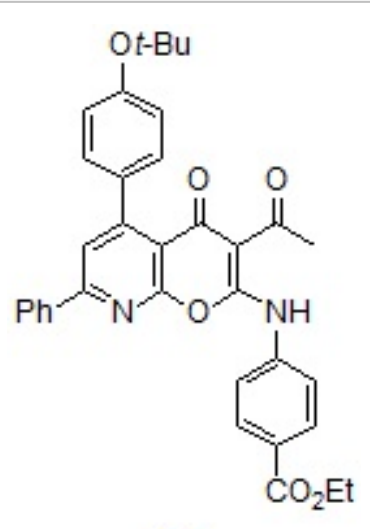

$6 \mathrm{db}$
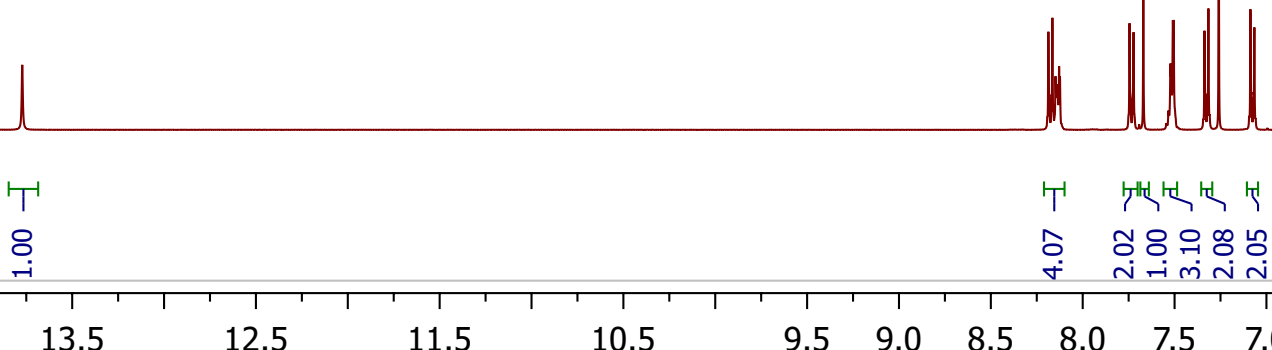

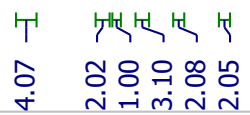

'T a nimin

i

$7.0 \underset{f 1}{6.5 p m)} 6.0$ 
Figure S98. Compound 6db, ${ }^{13} \mathrm{C}\left\{{ }^{1} \mathrm{H}\right\}$ NMR (101 MHz, $\left.\mathrm{CDCl}_{3}\right)$

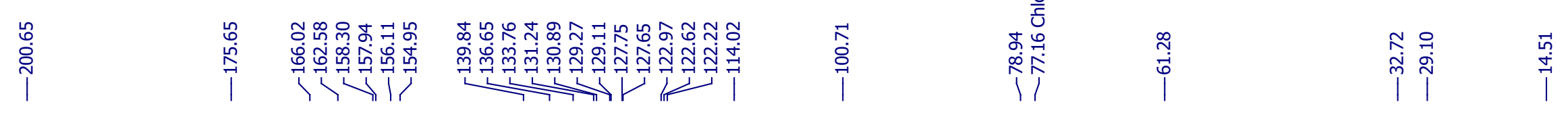

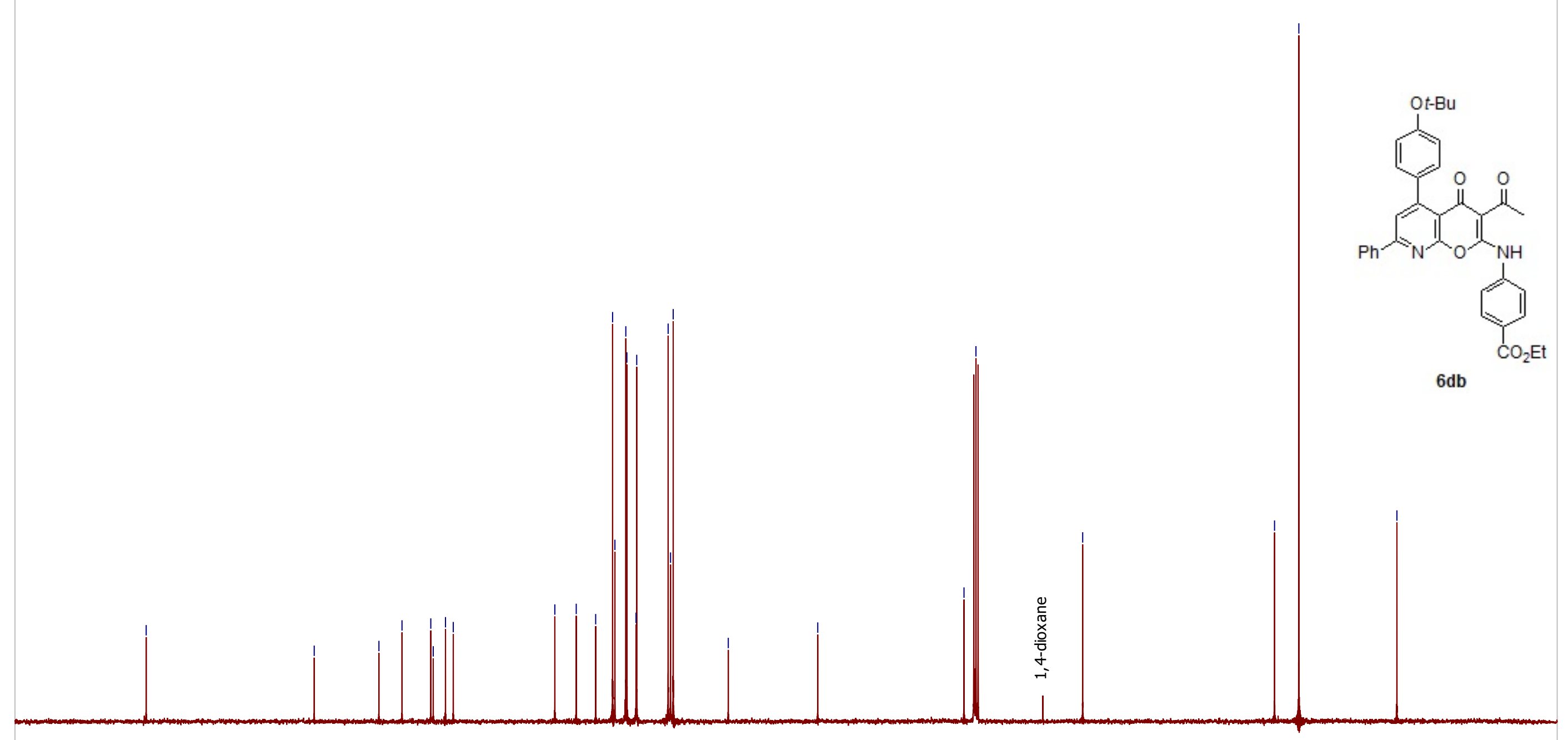

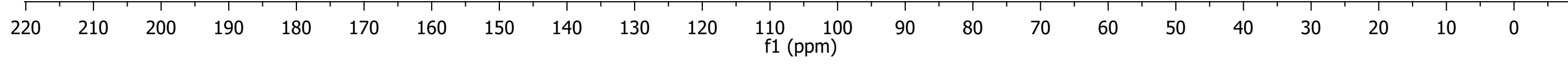




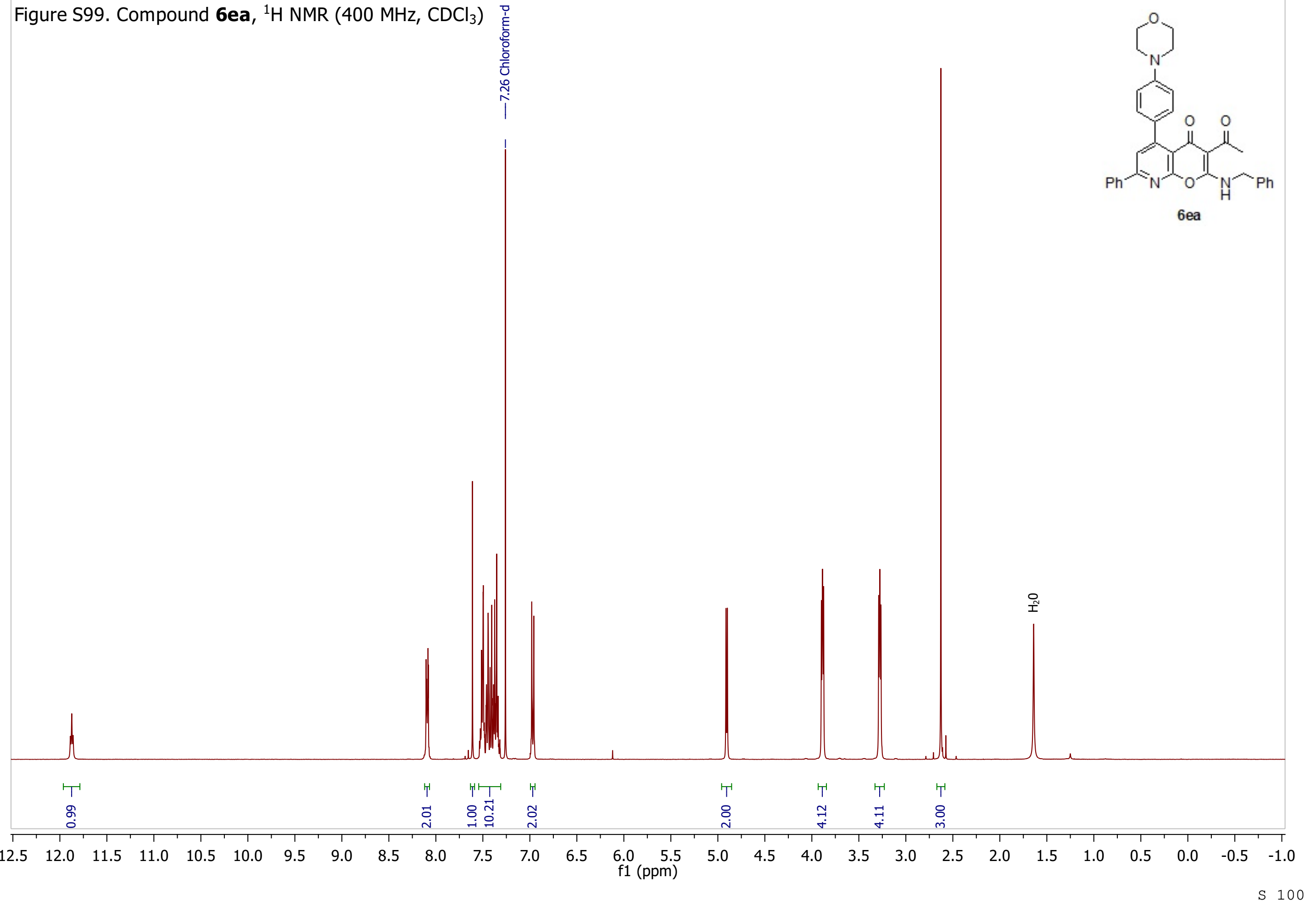


Figure S100. Compound 6ea, ${ }^{13} \mathrm{C}\left\{{ }^{1} \mathrm{H}\right\} \mathrm{NMR}\left(101 \mathrm{MHz}, \mathrm{CDCl}_{3}\right)$

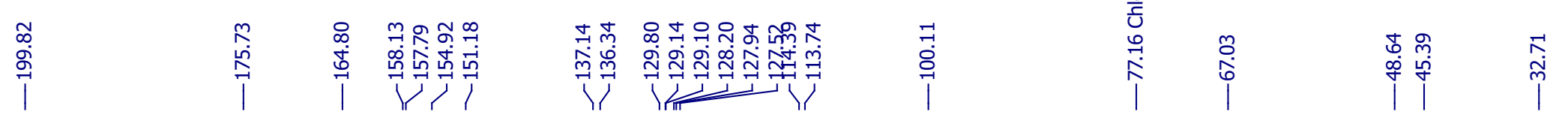
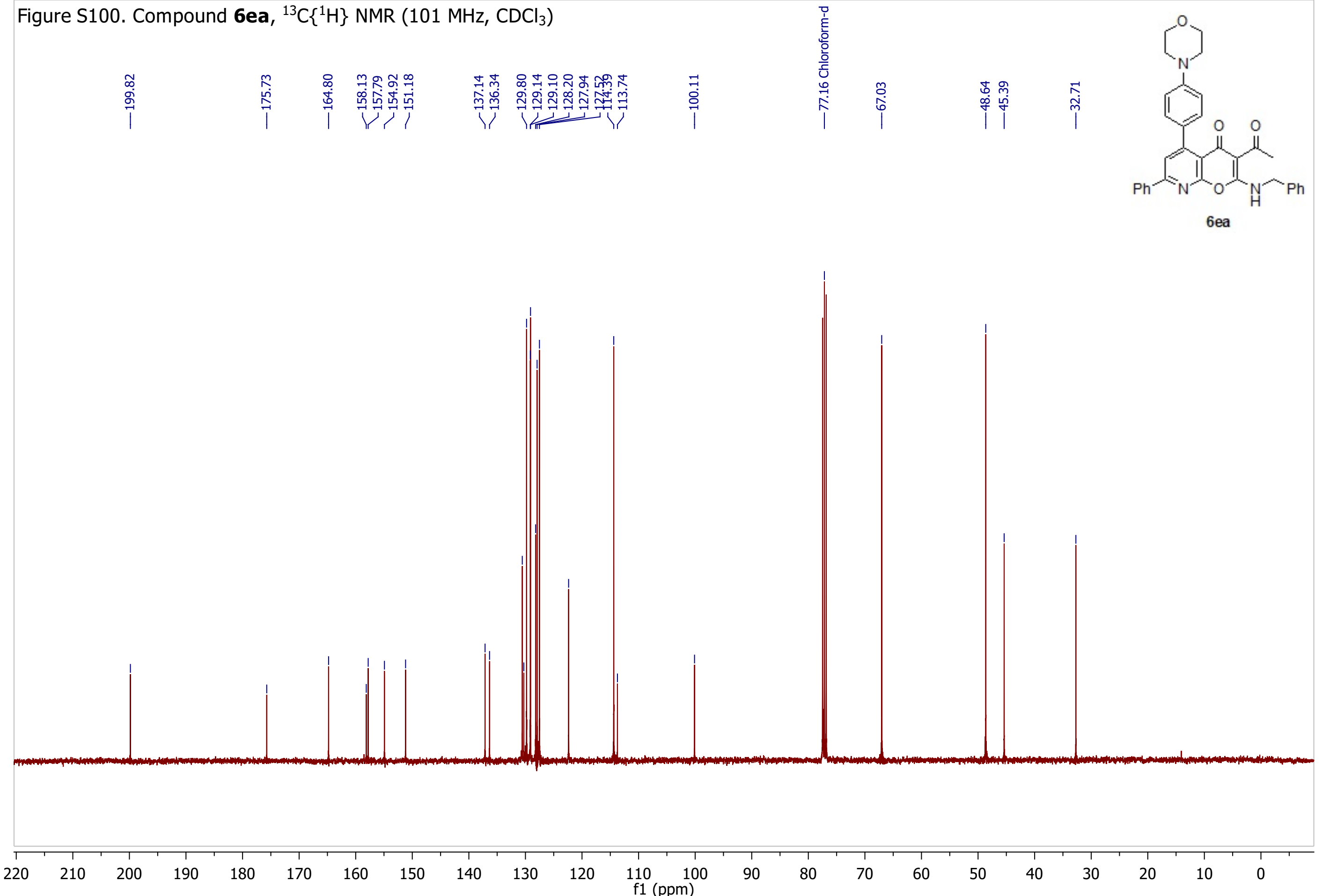
Figure S101. Compound 6eb, ${ }^{1} \mathrm{H}$ NMR (400 MHz, $\left.\mathrm{CDCl}_{3}\right)$

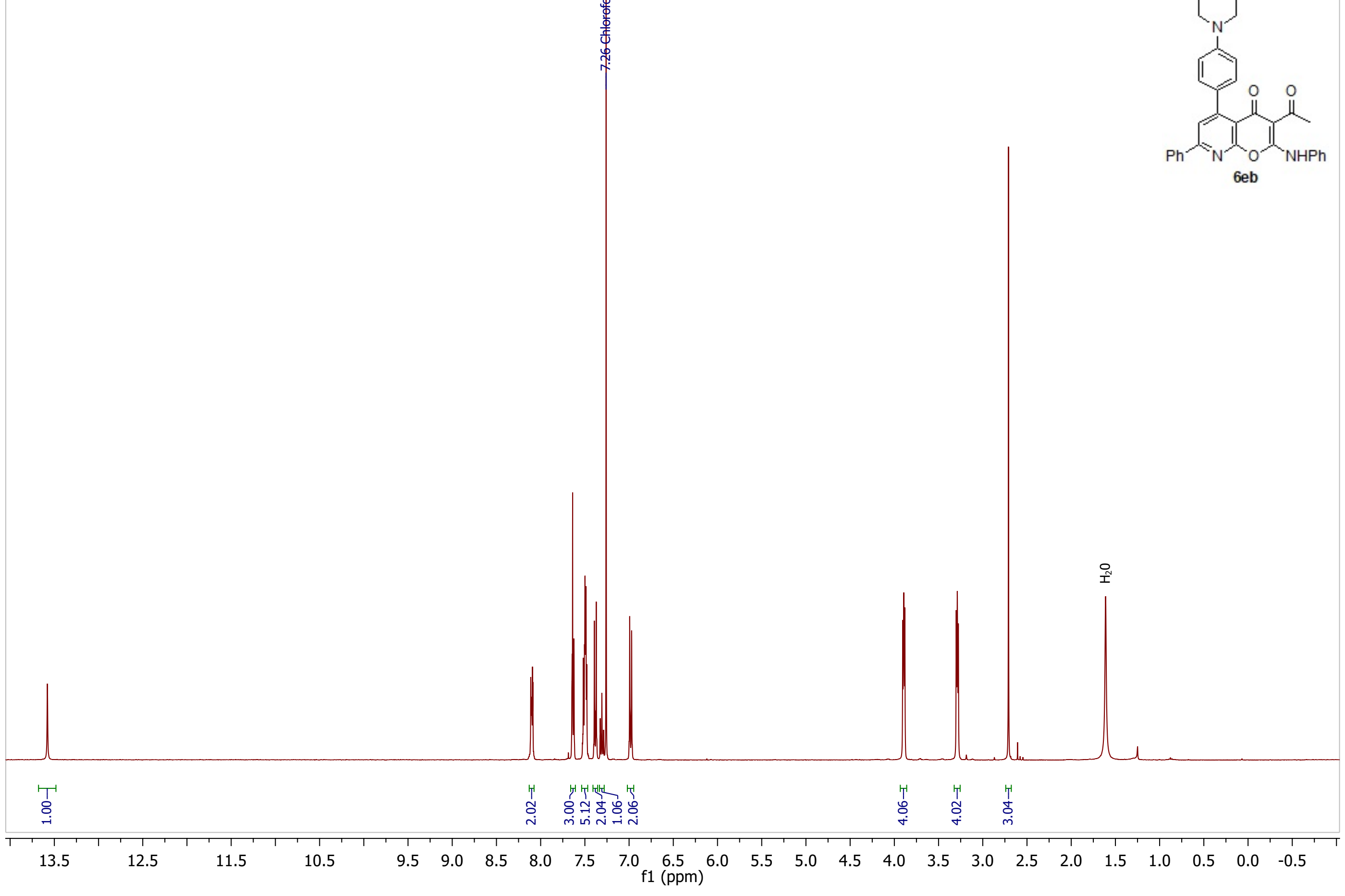


Figure S102. Compound 6eb, ${ }^{13} \mathrm{C}\left\{{ }^{1} \mathrm{H}\right\}$ NMR $\left(101 \mathrm{MHz}, \mathrm{CDCl}_{3}\right)$

| l|

$\underset{\substack{0 \\ \dot{b}}}{1}$

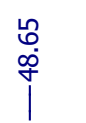

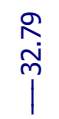
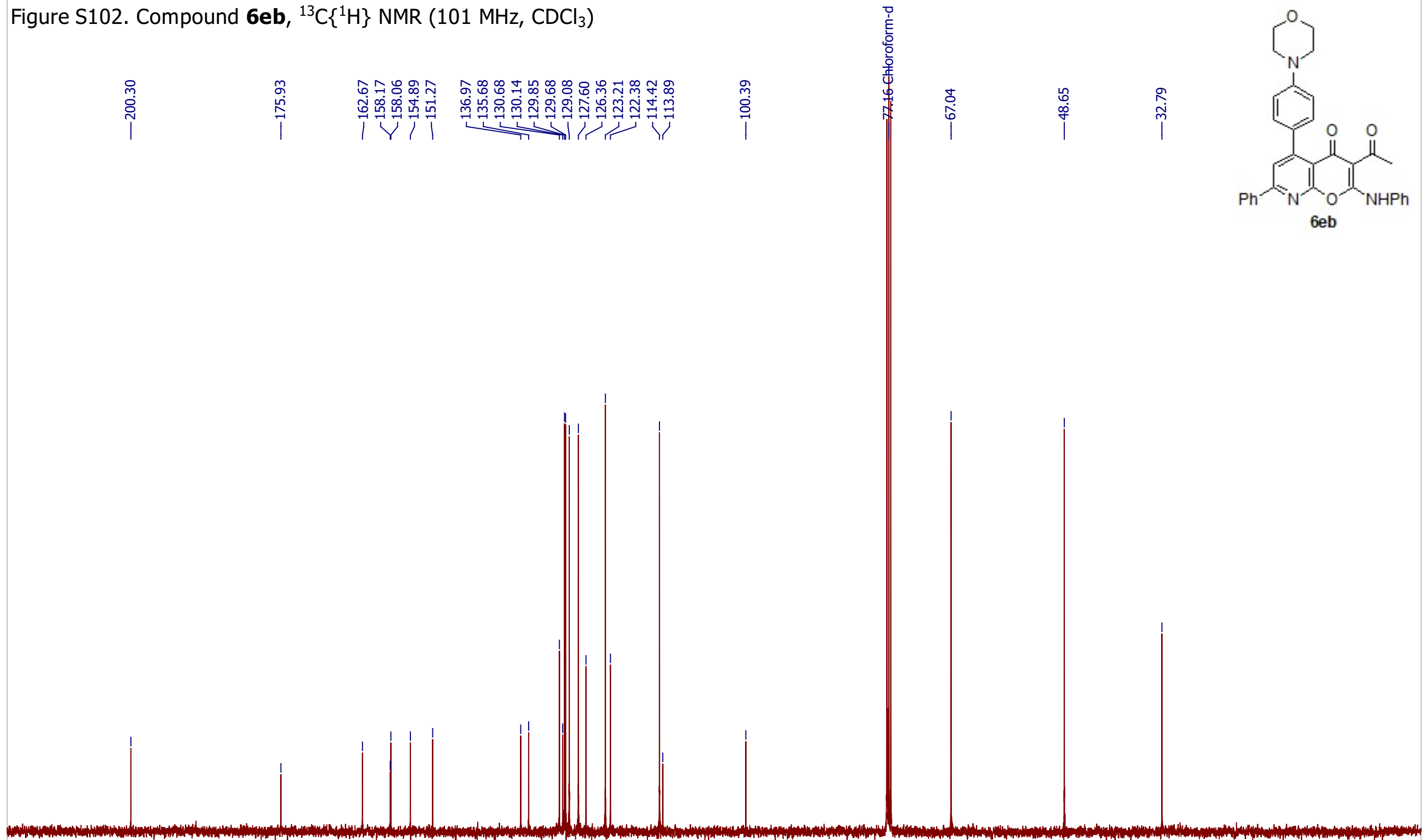
Figure S103. Compound 7a, ${ }^{1} \mathrm{H}$ NMR (400 MHz, $\left.\mathrm{CDCl}_{3}\right)$

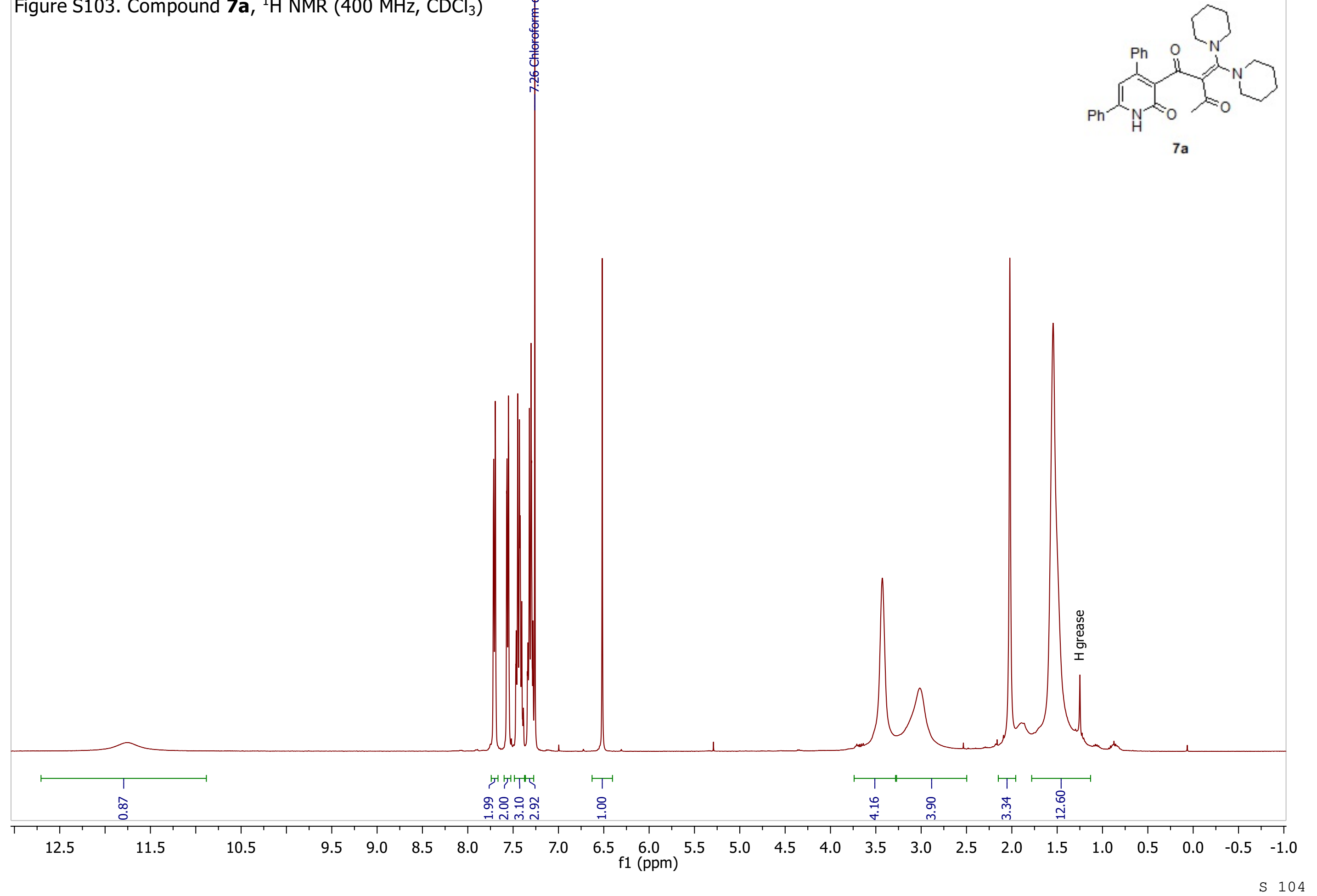


Figure S104. Compound 7a, ${ }^{13} \mathrm{C}\left\{{ }^{1} \mathrm{H}\right\}$ NMR (151 MHz, $\left.\mathrm{CDCl}_{3}\right)$
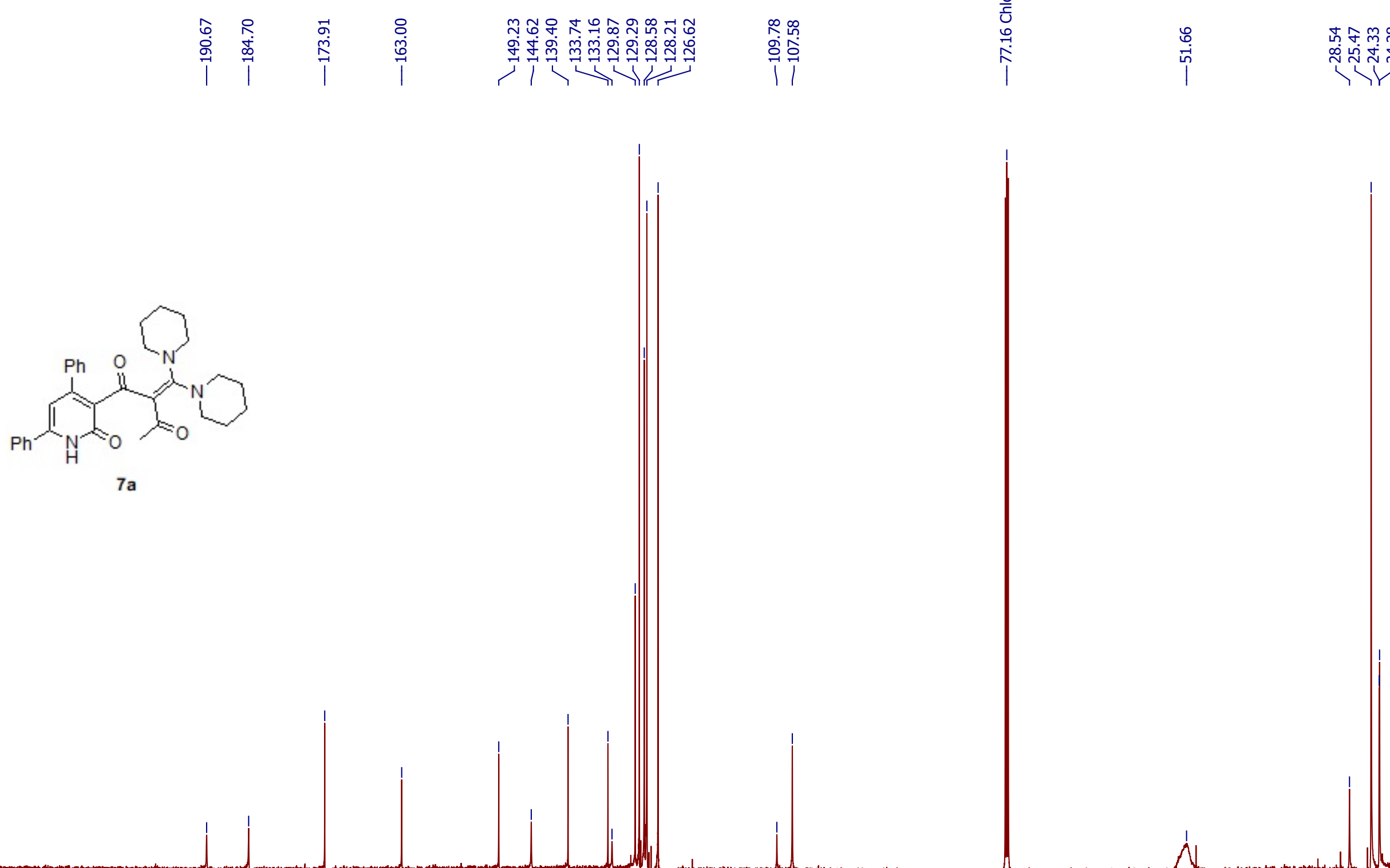


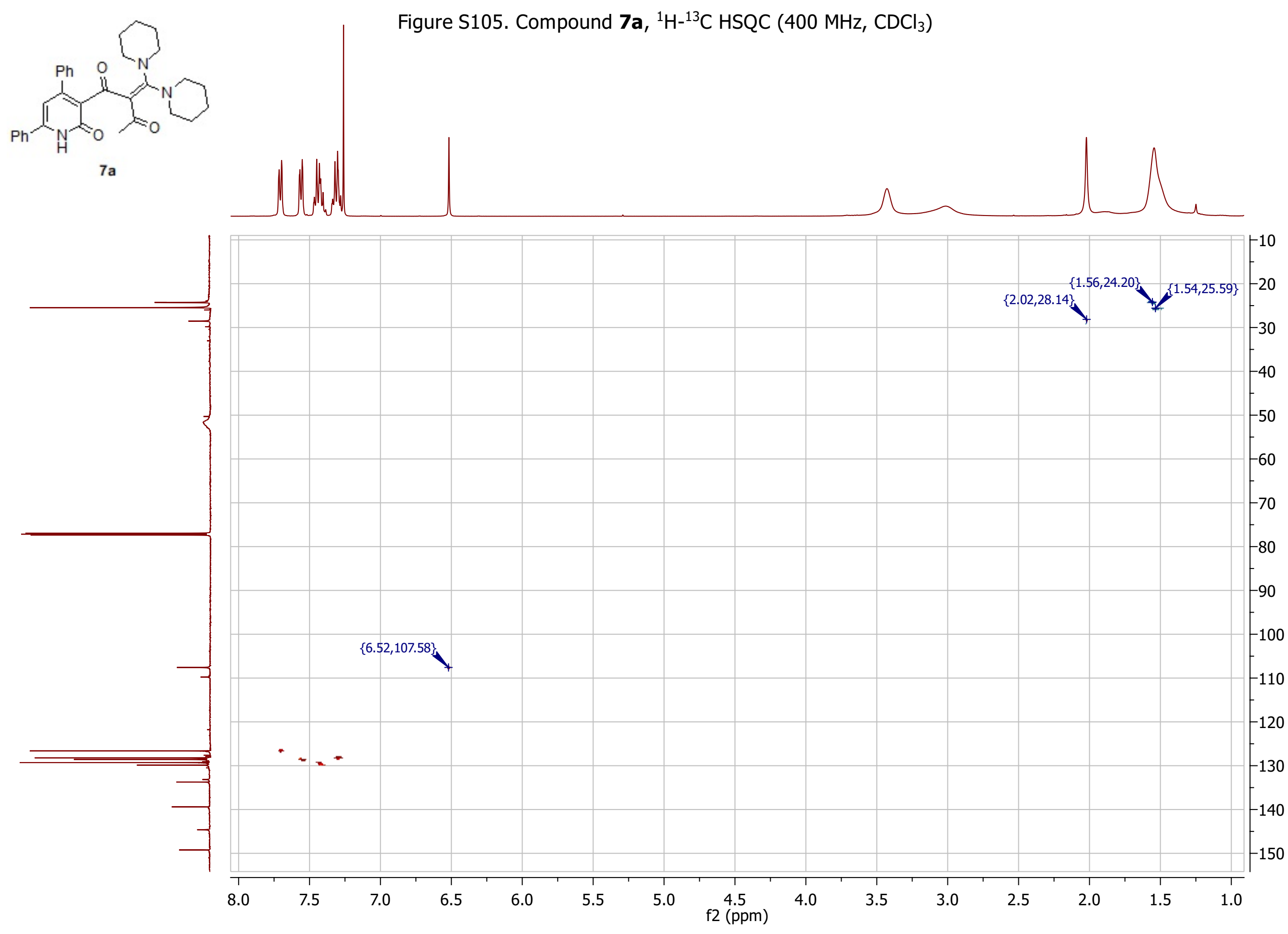




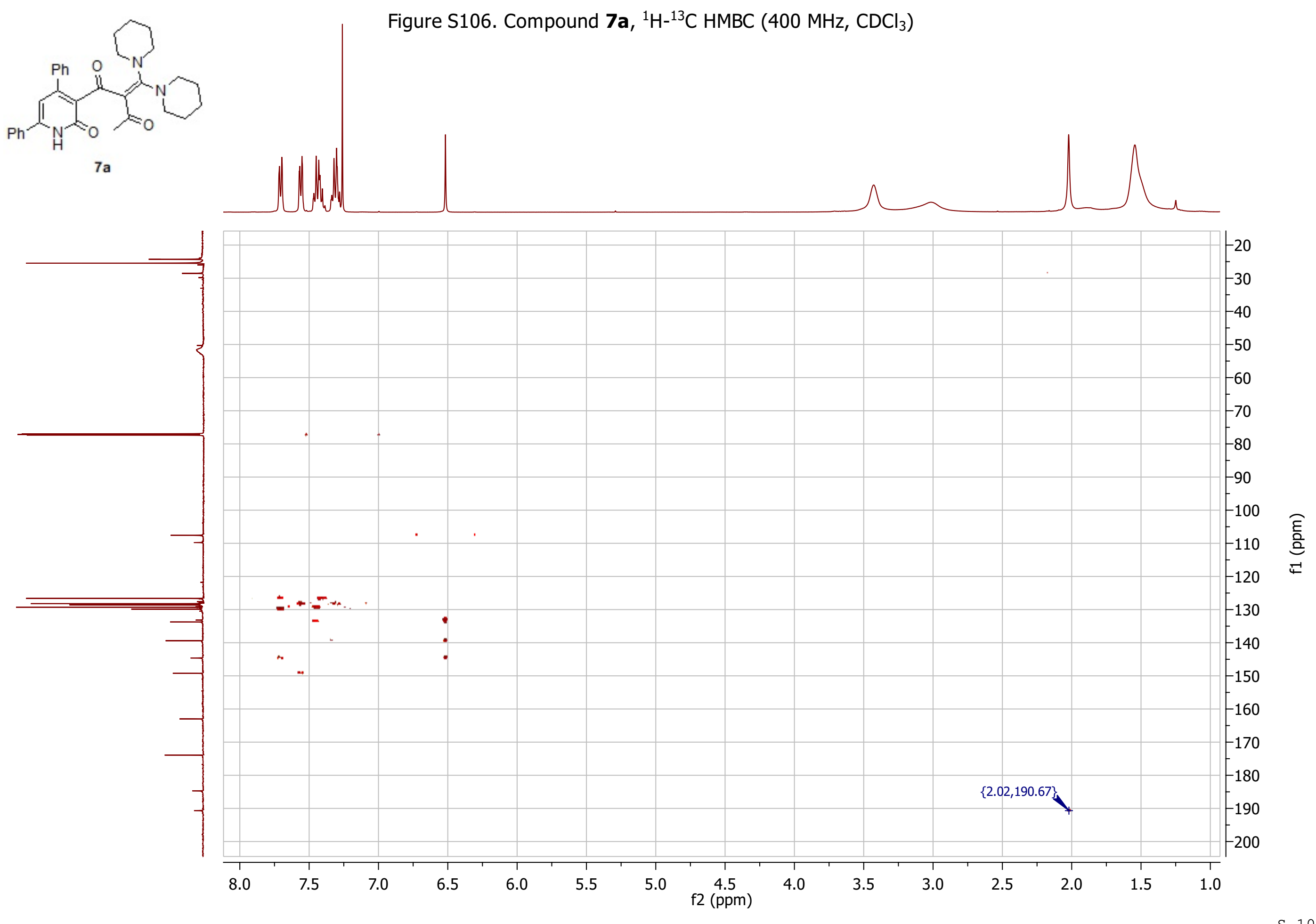


Figure S107. Compound 7b, ${ }^{1} \mathrm{H}$ NMR (400 MHz, CDCl 3 )

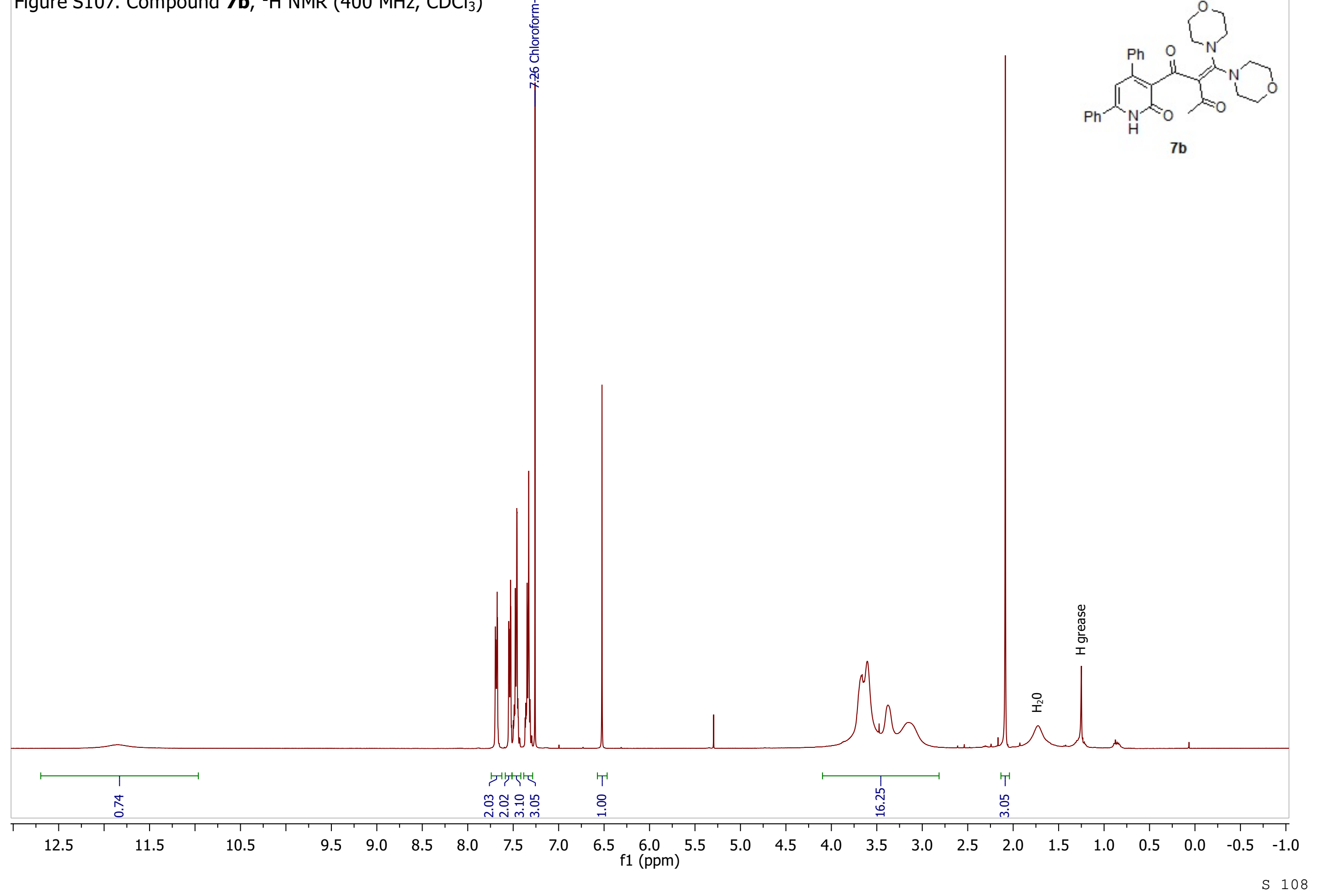


Figure S108. Compound 7b, ${ }^{13} \mathrm{C}\left\{{ }^{1} \mathrm{H}\right\} \mathrm{NMR}\left(101 \mathrm{MHz}, \mathrm{CDCl}_{3}\right)$

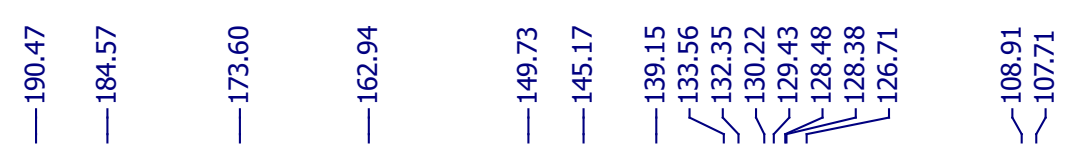

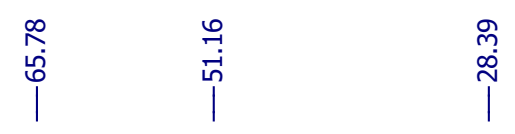

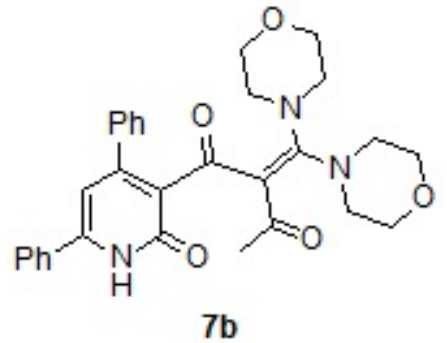

$\begin{array}{rlllllllllllllllllllllll}220 & 210 & 200 & 190 & 180 & 170 & 160 & 150 & 140 & 130 & 120 & \begin{array}{c}110 \\ \mathrm{f} 1(\mathrm{ppm})\end{array} & 90 & 80 & 70 & 60 & 50 & 40 & 30 & 20 & 10 & 0 & \end{array}$




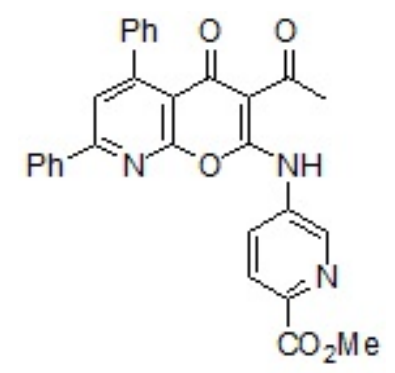

6an

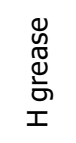

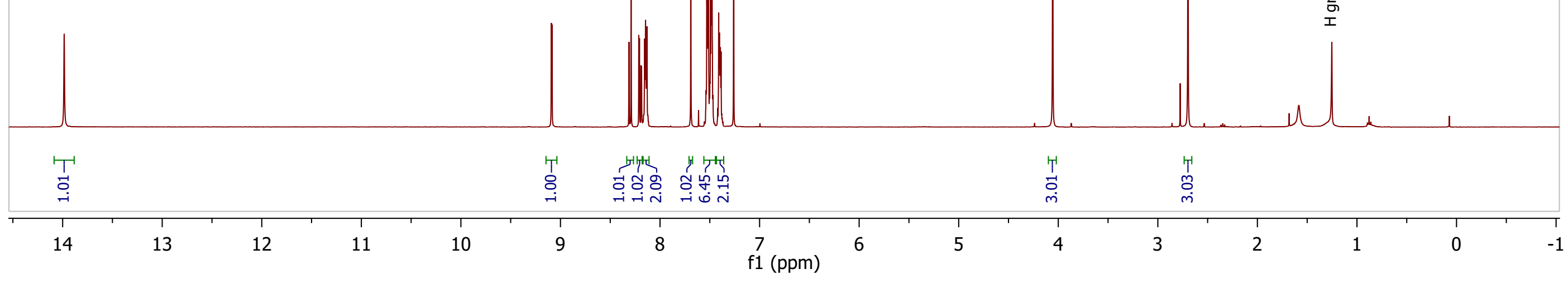


Figure S110. Compound 6an, ${ }^{13} \mathrm{C}\left\{{ }^{1} \mathrm{H}\right\}$ NMR $\left(101 \mathrm{MHz}, \mathrm{CDCl}_{3}\right)$

$\begin{array}{lll}0 & 0 \\ 0 & 0\end{array}$

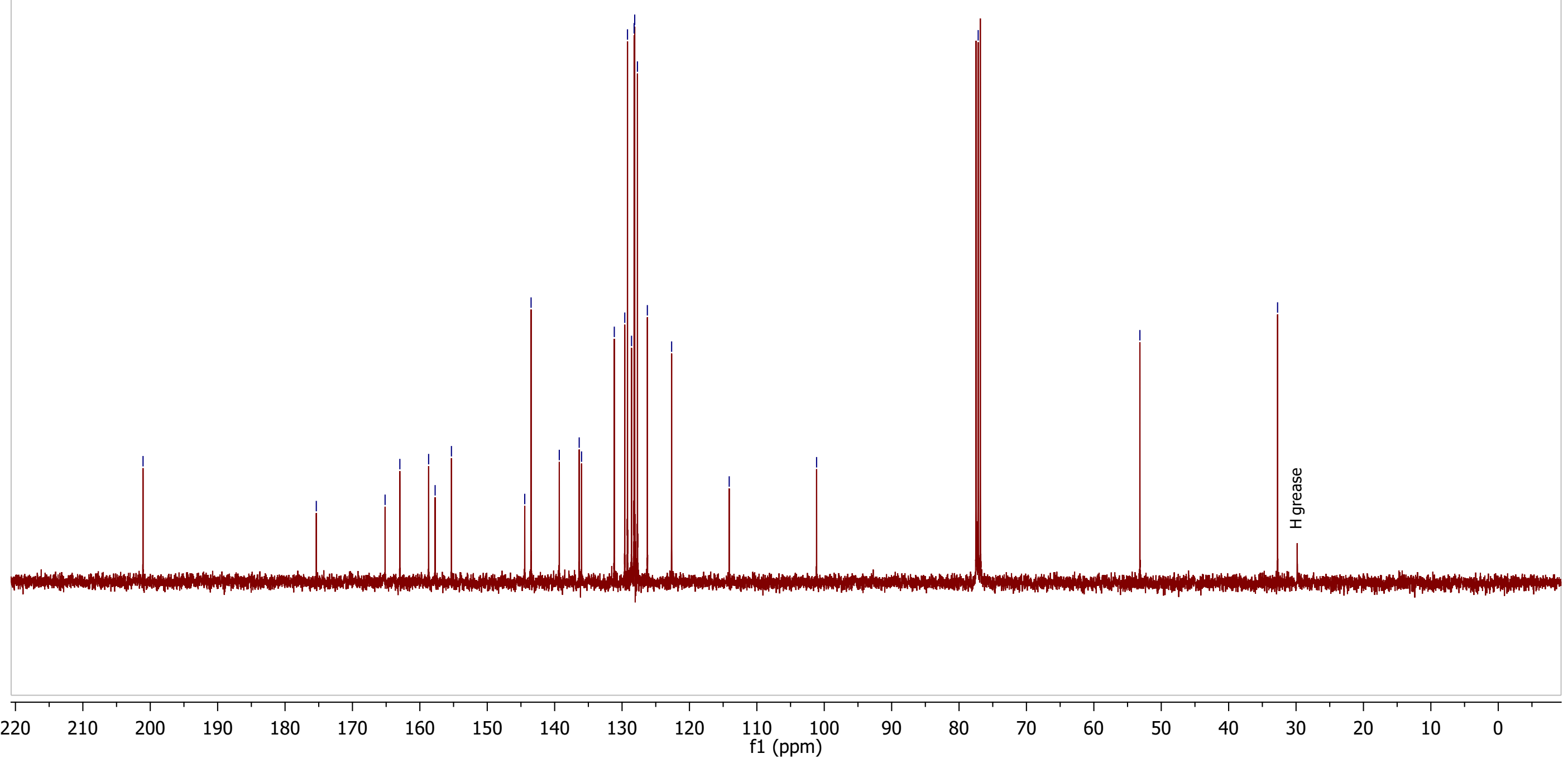




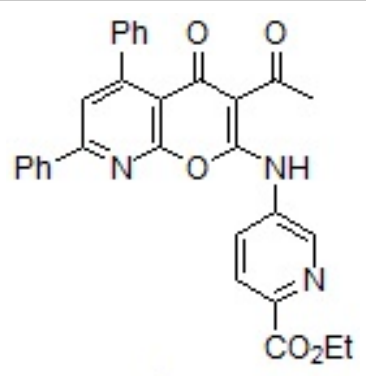

$6 a$

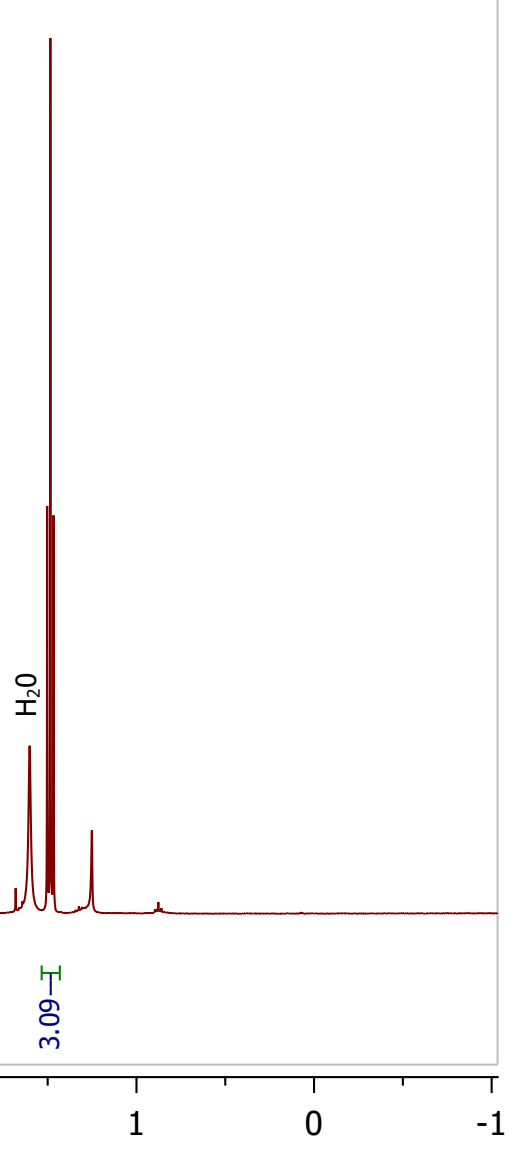


Figure S112. Compound 6ao, ${ }^{13} \mathrm{C}\left\{{ }^{1} \mathrm{H}\right\}$ NMR $\left(101 \mathrm{MHz}, \mathrm{CDCl}_{3}\right)$

| lon

i

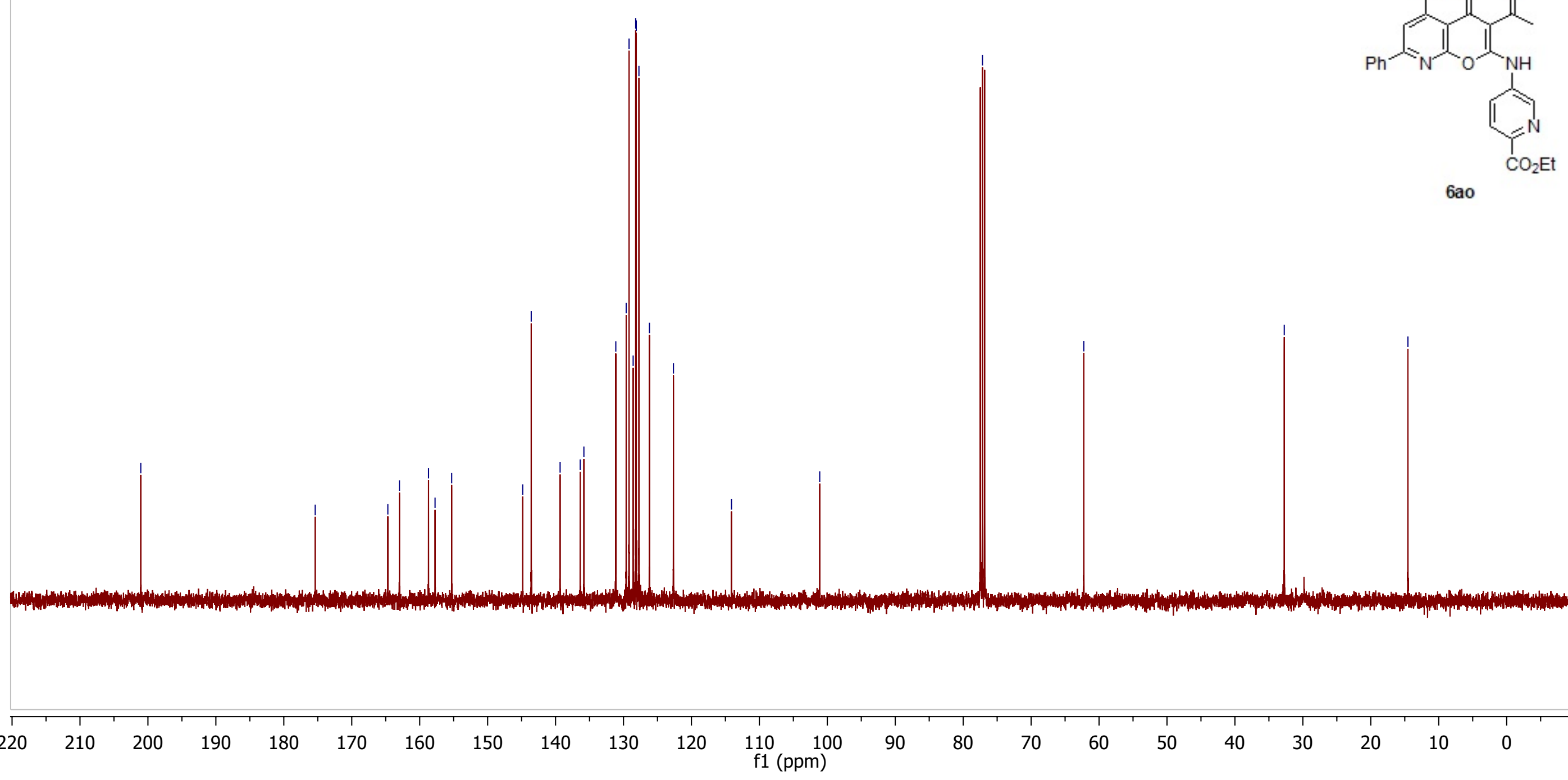


Figure S113. Compound 6ap, ${ }^{1} \mathrm{H}$ NMR (400 MHz, $\left.\mathrm{CDCl}_{3}\right)$

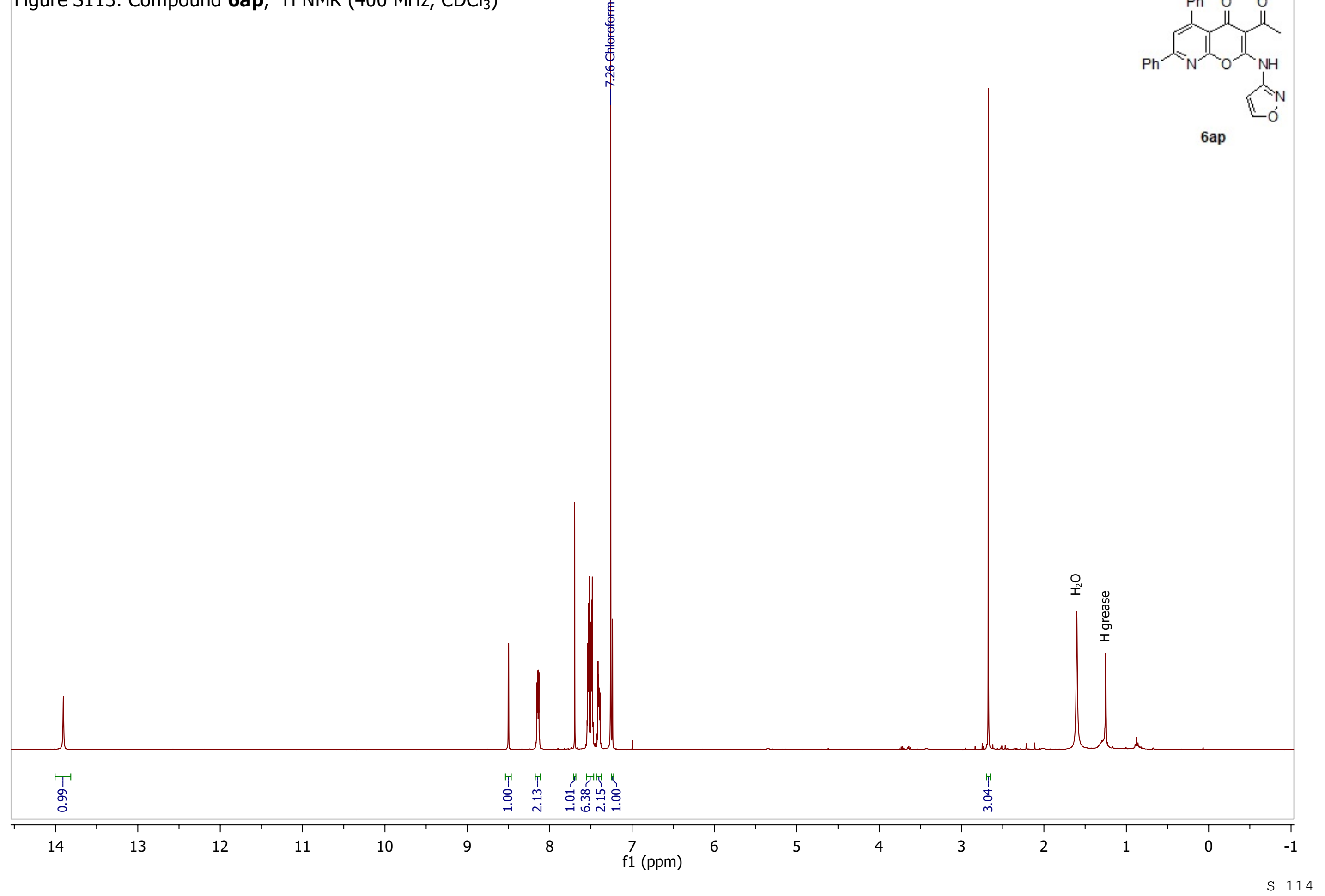


Figure S114. Compound 6ap, ${ }^{13} \mathrm{C}\left\{{ }^{1} \mathrm{H}\right\}$ NMR $\left(126 \mathrm{MHz}, \mathrm{CDCl}_{3}\right)$

|

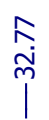

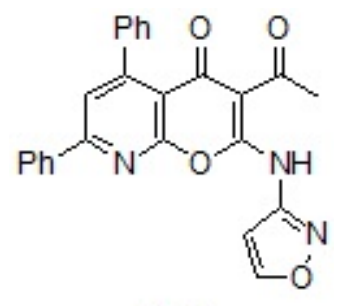

6ap

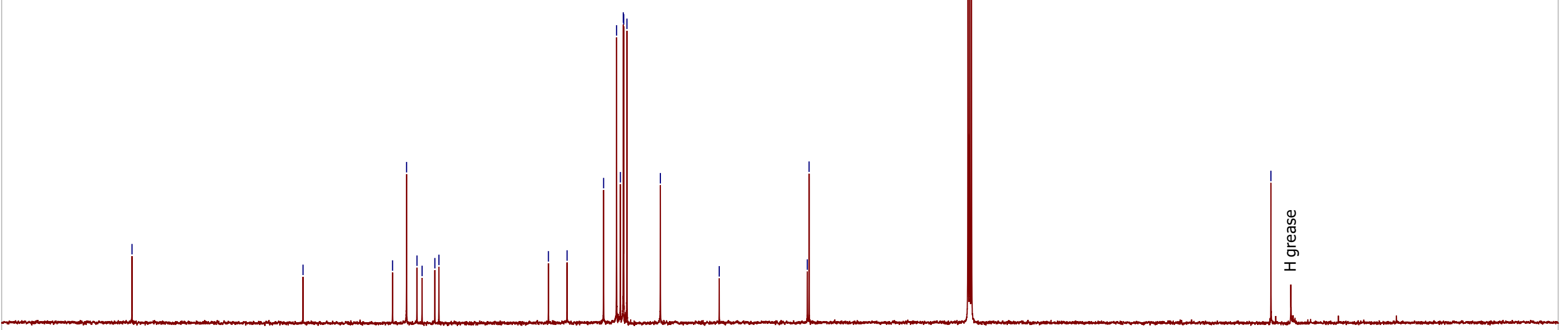




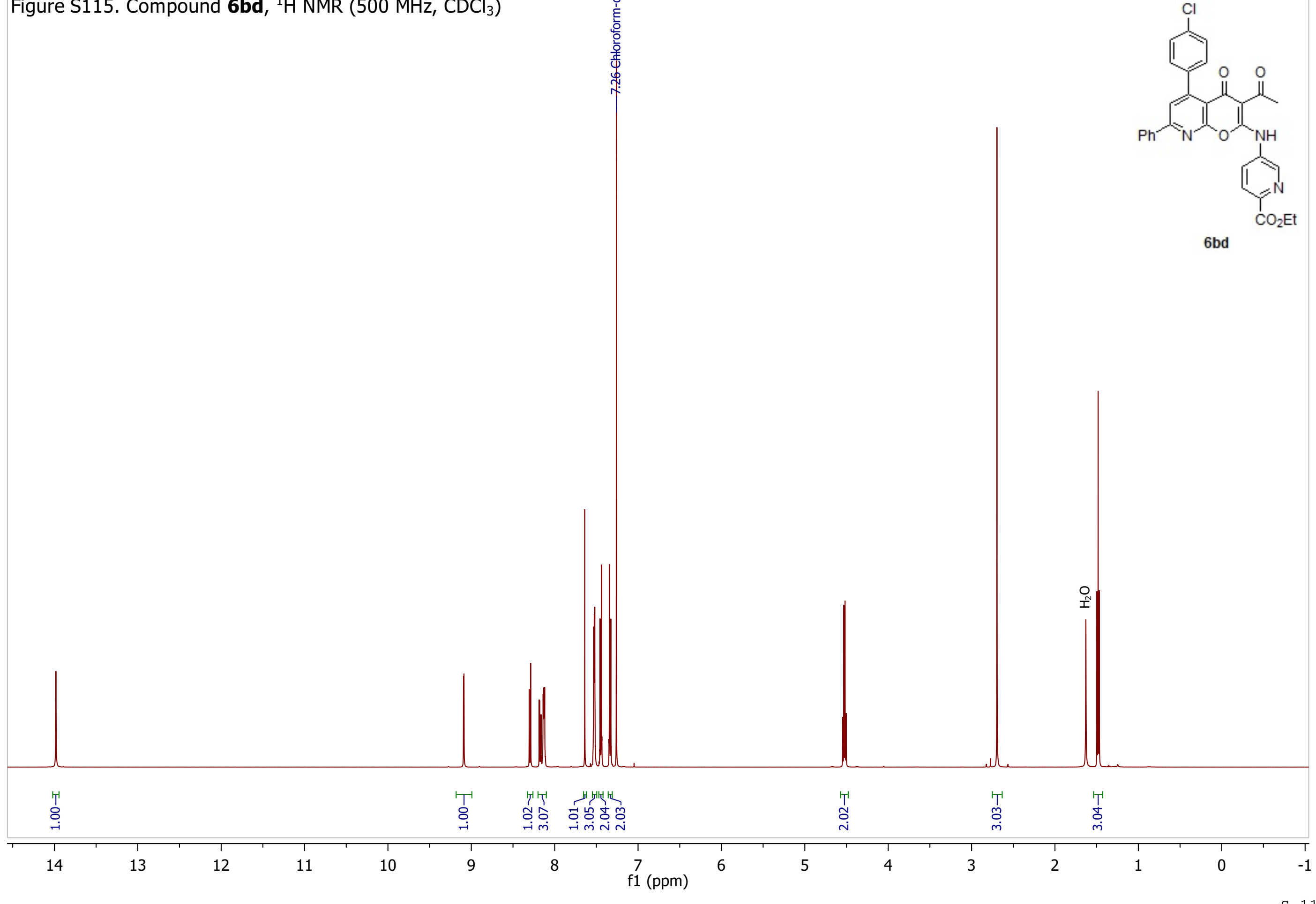


Figure S116. Compound 6bd, ${ }^{13} \mathrm{C}\left\{{ }^{1} \mathrm{H}\right\} \mathrm{NMR}\left(126 \mathrm{MHz}, \mathrm{CDCl}_{3}\right)$

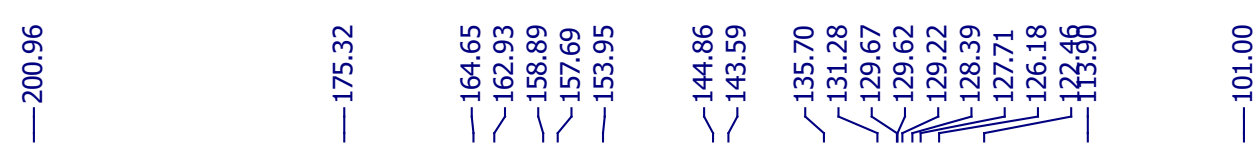

i.

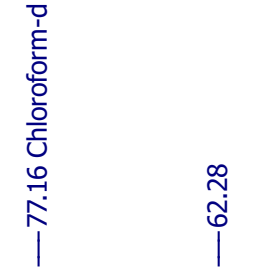

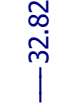

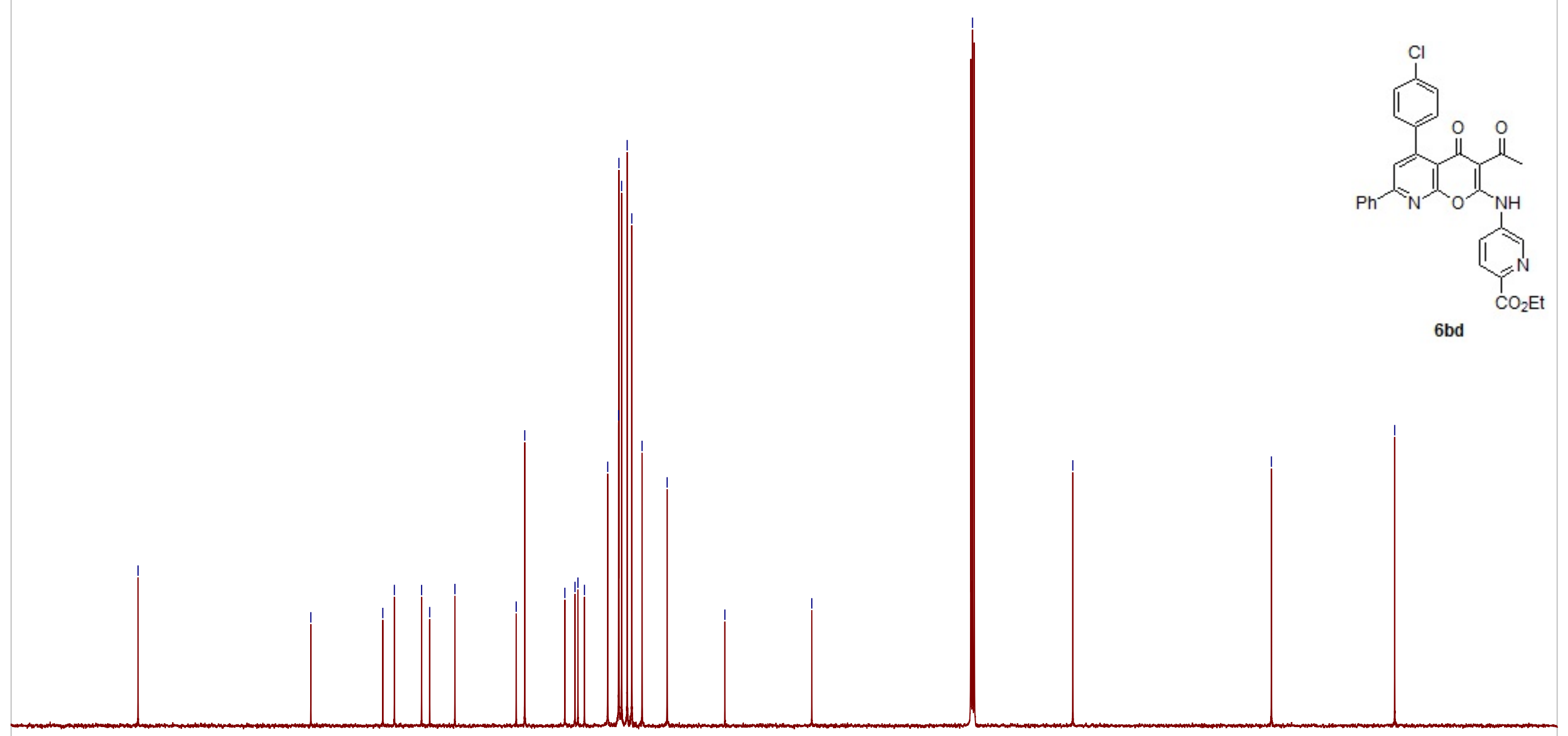




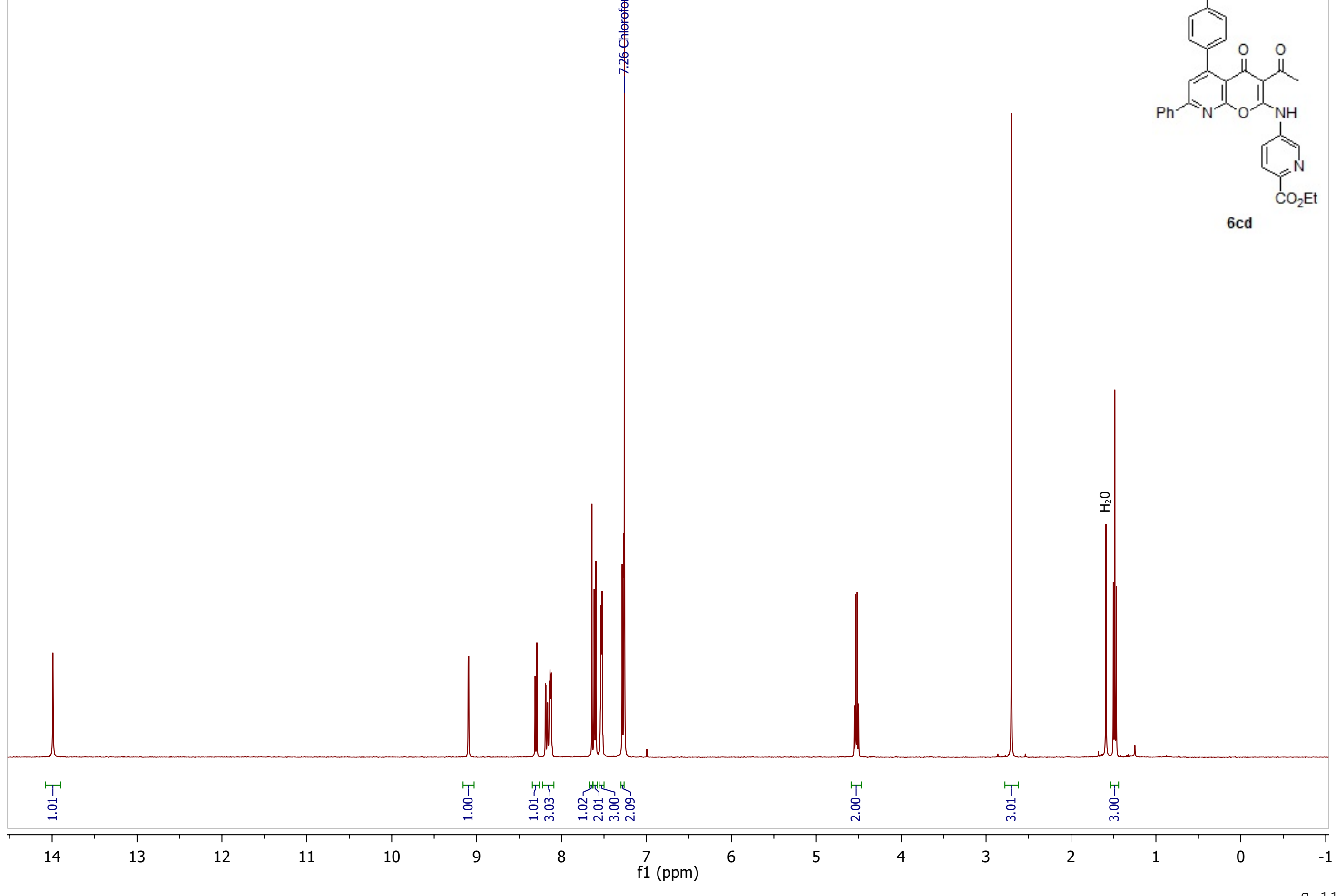


Figure S118. Compound $\mathbf{6 c d},{ }^{13} \mathrm{C}\left\{{ }^{1} \mathrm{H}\right\} \mathrm{NMR}\left(101 \mathrm{MHz}, \mathrm{CDCl}_{3}\right)$
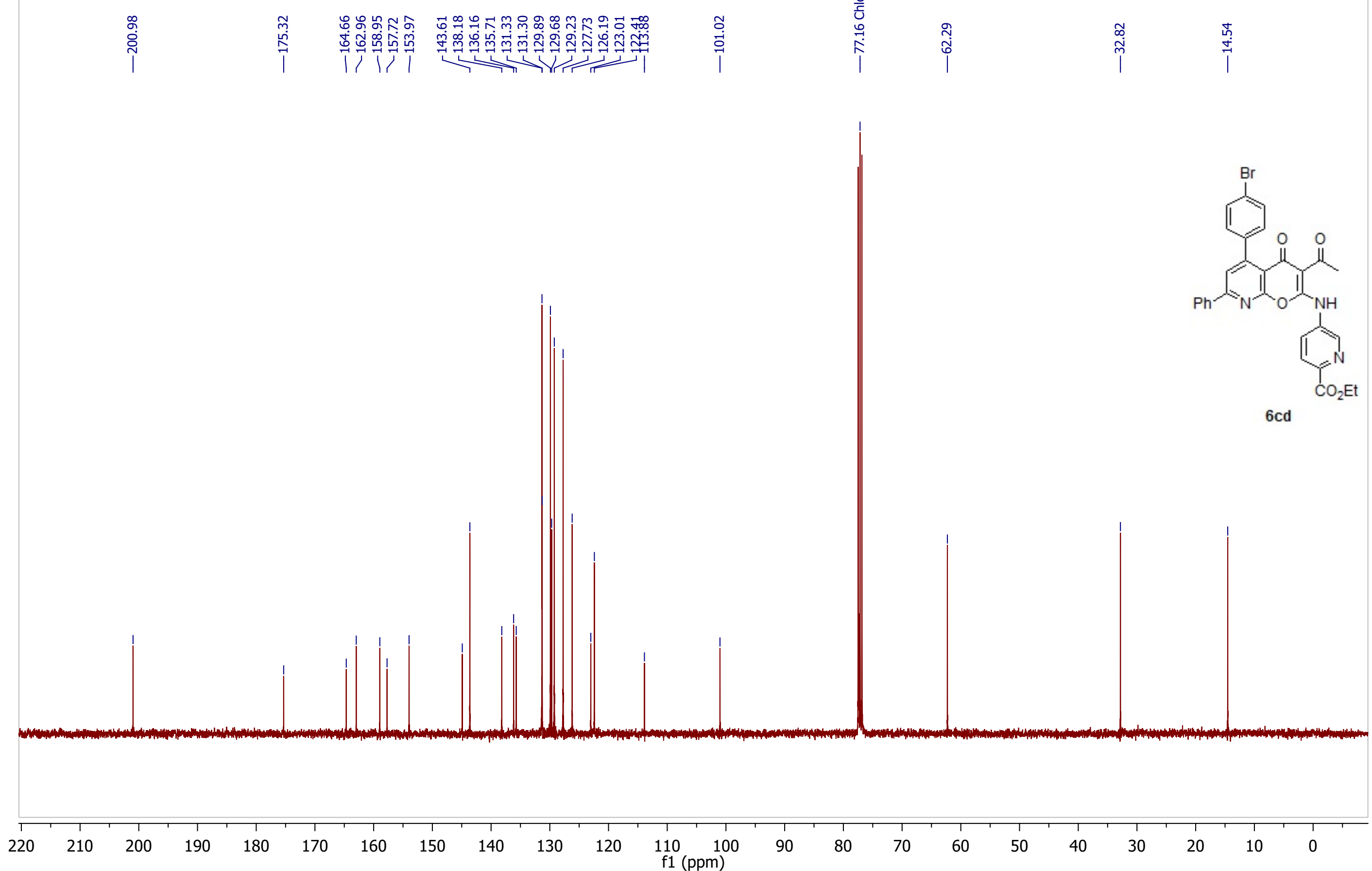
Figure S119. Compound $\mathbf{6 d c},{ }^{1} \mathrm{H}$ NMR $\left(400 \mathrm{MHz}, \mathrm{CDCl}_{3}\right)$

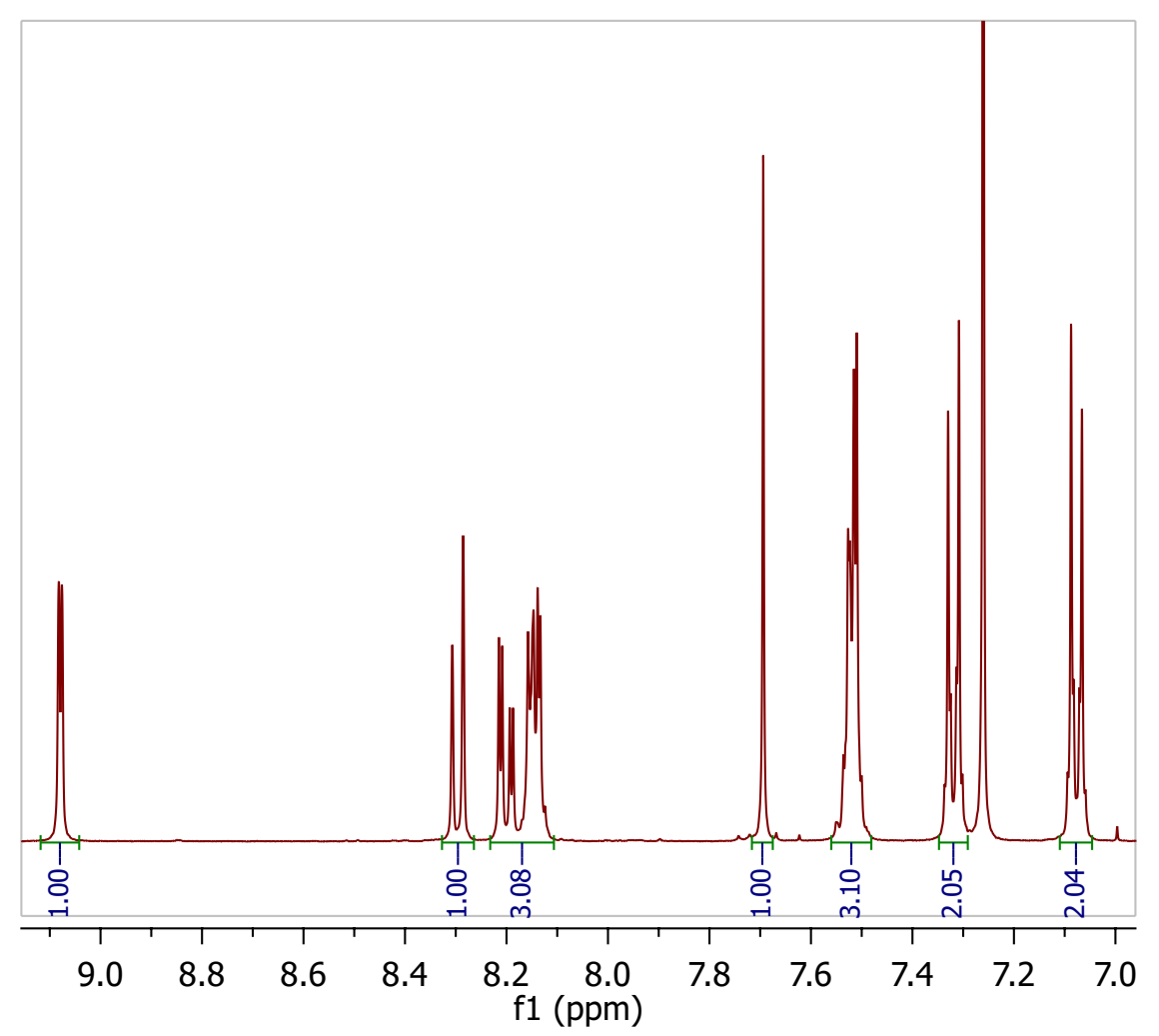

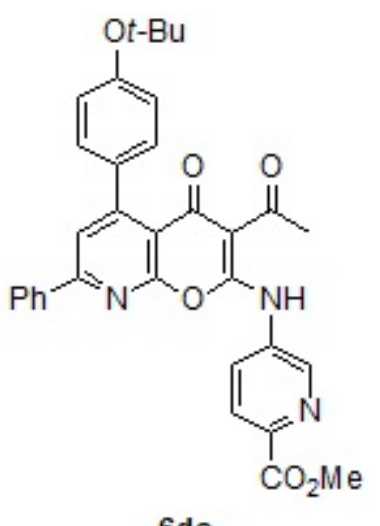

$6 \mathrm{dc}$

$\stackrel{\circ}{\mathbf{x}^{\prime}}$

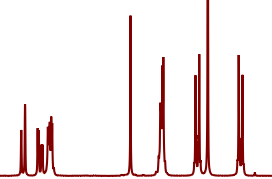

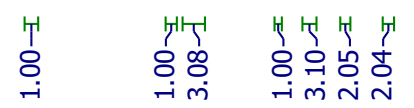

$\stackrel{m}{m}$

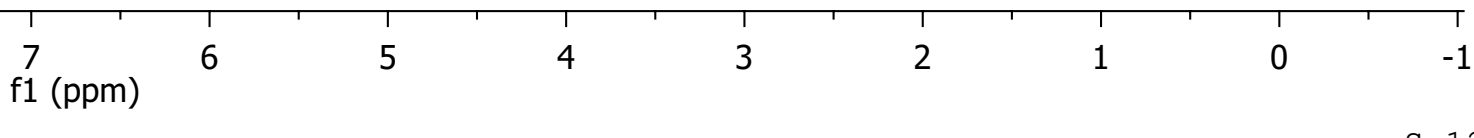


Figure S120. Compound $\mathbf{6 d c},{ }^{13} \mathrm{C}\left\{{ }^{1} \mathrm{H}\right\}$ NMR $\left(101 \mathrm{MHz}, \mathrm{CDCl}_{3}\right)$

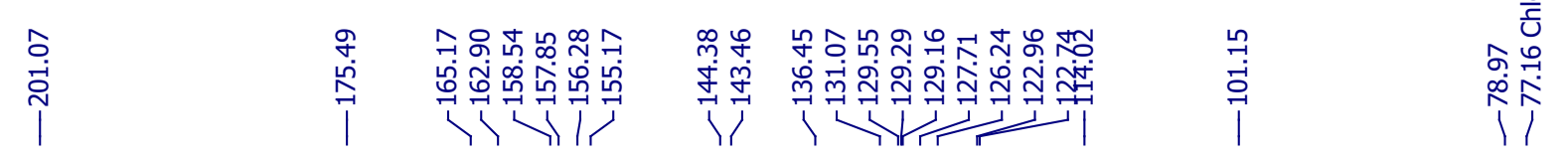

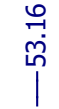

ธิ

กิ่

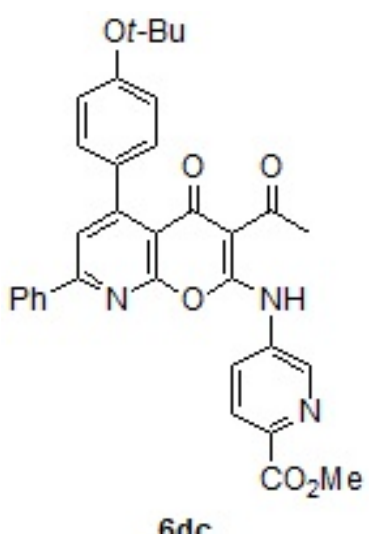

$6 d c$

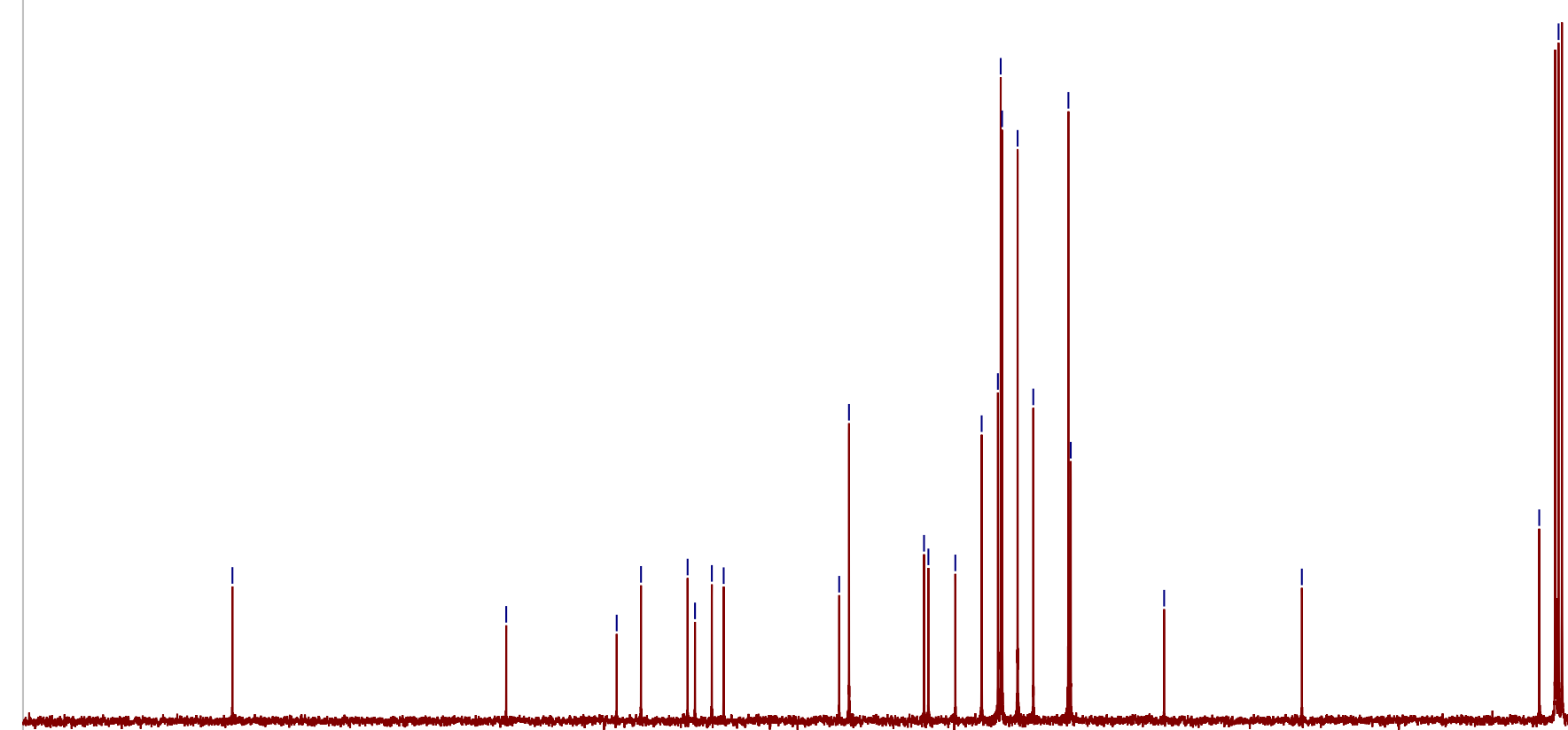




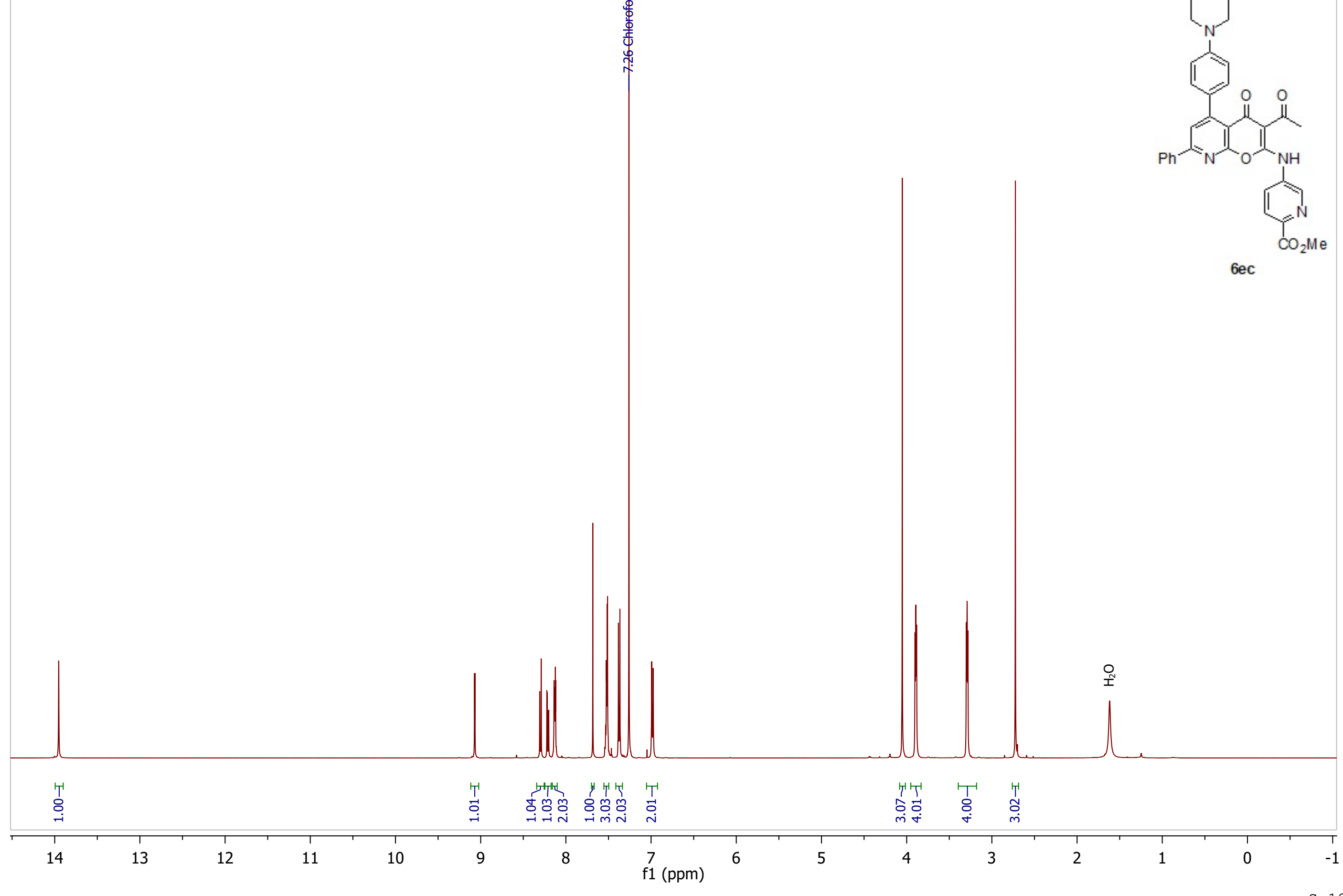


Figure S122. Compound 6ec, ${ }^{13} \mathrm{C}\left\{{ }^{1} \mathrm{H}\right\} \mathrm{NMR}\left(126 \mathrm{MHz}, \mathrm{CDCl}_{3}\right)$

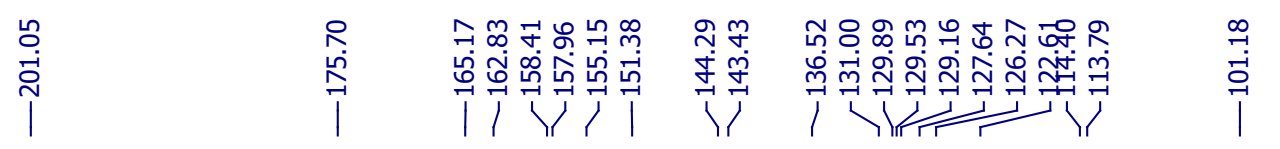

$\stackrel{8}{\stackrel{0}{6}}$

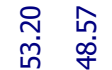

ஸึ

$\stackrel{\substack{\hat{i} \\ \stackrel{1}{m}}}{1}$

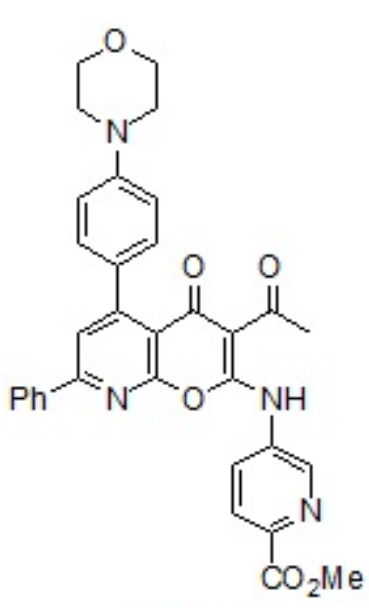

$6 e c$

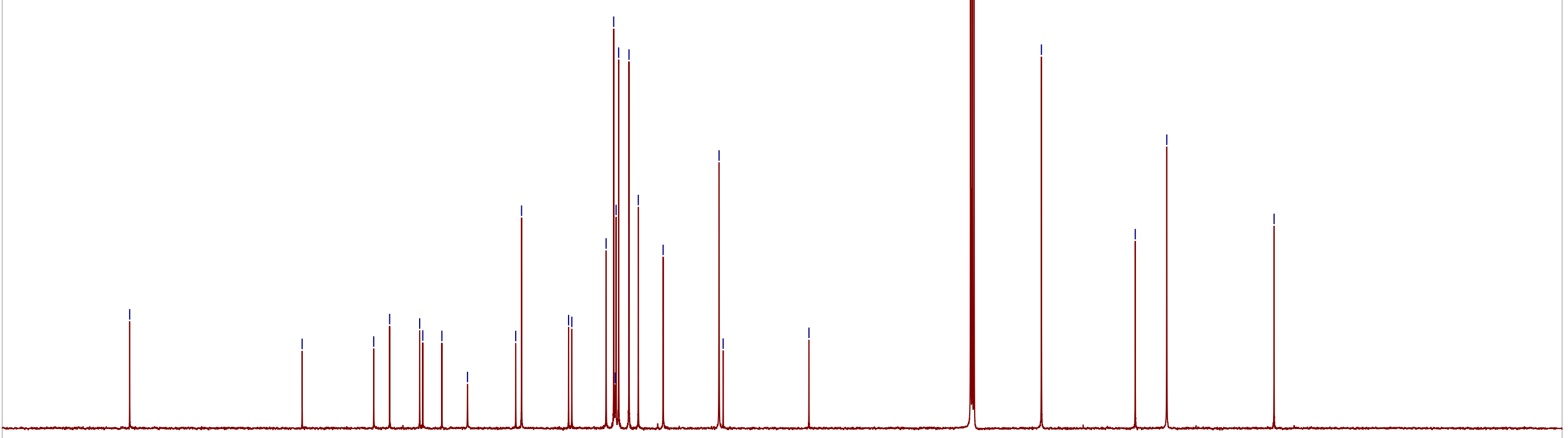

